\title{
Stimuli-Responsive Cycloaurated 'OFF-ON' Switchable Anion Transporters
}

Mohamed Fares, Xin Wu, Deepthi Ramesh, William Lewis, Paul Keller, Ethan Howe, Ricardo Pérez-Tomás, Philip Gale

Submitted date: 03/05/2020 - Posted date: 05/05/2020

Licence: CC BY-NC-ND 4.0

Citation information: Fares, Mohamed; Wu, Xin; Ramesh, Deepthi; Lewis, William; Keller, Paul; Howe, Ethan; et al. (2020): Stimuli-Responsive Cycloaurated 'OFF-ON'Switchable Anion Transporters. ChemRxiv. Preprint. https://doi.org/10.26434/chemrxiv.12235880.v1

Anion transporters have shown potential application as anti-cancer agents that function by disrupting homeostasis and triggering cell death. In this research article we report switchable anion transport by gold complexes of anion transporters that are 'switched on' in the presence of the reducing agent GSH by decomplexation of gold. GSH is found in higher concentrations in tumours than in healthy tissue and hence offers a strategy to target these systems to tumours.

File list (2)

Paper.pdf (5.30 MiB)

view on ChemRxiv - download file 


\title{
Stimuli-responsive cycloaurated 'OFF-ON' switchable anion
} transporters

\author{
Mohamed Fares, ${ }^{[a, b]}$ Xin Wu, ${ }^{[a]}$ Deepthi Ramesh, ${ }^{[c]}$ William Lewis, ${ }^{[a]}$ Paul A. Keller, ${ }^{[b]}$ Ethan N.W. \\ Howe, ${ }^{[a, d] *}$ Ricardo Pérez-Tomás, ${ }^{[c] *}$ and Philip A. Gale ${ }^{[\mathrm{a}] *}$
}

[a] M. Fares, Dr. X. Wu, Dr. W. Lewis, Dr E.N.W. Howe, Prof. P. A. Gale. School of Chemistry, The University of Sydney

Sydney, New South Wales 2006 (Australia)

E-mail: philip.gale@sydney.edu.au

[b] M. Fares, Prof. P. A. Keller

School of Chemistry \& Molecular Bioscience, Molecular Horizons, University of Wollongong, and Illawarra Health \& Medical Research Institute Wollongong, NSW 2522, Australia

[c] D. Ramesh, Prof. R. Pérez-Tomás

Faculty of Medicine \& Health Sciences. Department of Pathology and Experimental Therapeutics, Cancer Cell Biology Research Group

University of Barcelona, Barcelona, Spain

E-mail: rperez@ub.edu

[a] Dr E.N.W. Howe

GlaxoSmithKline, GSK Jurong

1 Pioneer Sector 1, Singapore 628413

E-mail: ethan.n.howe@gsk.com

Supporting information for this article is given via a link at the end of the document.

\begin{abstract}
Anion transporters have shown potential application as anti-cancer agents that function by disrupting homeostasis and triggering cell death. In this research article we report switchable anion transport by gold complexes of anion transporters that are 'switched on' in the presence of the reducing agent GSH by decomplexation of gold. GSH is found in higher concentrations in tumours than in healthy tissue and hence offers a strategy to target these systems to tumours.
\end{abstract}

\section{Introduction}

Biomimetic stimuli-responsive systems activated under particular physical, chemical or biological conditions have recently gained increasing attention and represent a promising therapeutic approach due to their selective and controllable action. ${ }^{[1]}$ These smart systems can be switched on under specific conditions, including the presence of certain proteins, redox states or chemical signals (such as changes in $\mathrm{pH}$ ). ${ }^{[2]}$ In the field of anion recognition, $\mathrm{pH}$-responsive, redox-switchable and photophysical controllable anion receptors have been developed..$^{[3]}$ These switchable anion receptors have potential for use in a range of applications including ion sensing, catalysis and can be used as potential drug candidates in medicinal chemistry. ${ }^{[4]}$

Transmembrane ion transport is a fundamental biological process and is involved in diverse physiological functions including, neuroexcitation, muscle contraction, cell migration and proliferation, and maintaining cellular $\mathrm{pH}$, membrane potential and cellular secretions. ${ }^{[5]}$ Channelopathies are a group of diseases characterised by impaired ion channel function, including cystic fibrosis, epilepsy and cancer. ${ }^{[6]}$ Anionophores are small molecules that are capable of transporting anions and they demonstrated cytotoxic effects against stem cancer cells by facilitating the transport of chloride and bicarbonate anions so changing the cellular $\mathrm{pH}$, causing cellular differentiation and death. ${ }^{[4 c, 7]} \mathrm{A}$ recent mechanistic study of cancer death showed that the induction of apoptosis and inhibition of autophagy by particular anion transporters are due to the cellular osmotic stress which is caused by increasing cellular ion concentration. ${ }^{[8]}$ This promotes the generation of reactive oxygen species (ROS) and activates caspase-dependent apoptosis. In addition to this mechanism, some anion transporters are known to increase the lysosomal $\mathrm{pH}$ by promoting the transport of protons and chloride ions leading to disruption of autophagy. ${ }^{[8]}$

Glutathione $(\mathrm{GSH})$ is a tripeptide and forms, with glutathione disulfide (GSSG), a redox complex that helps maintain normal oxidative physiological conditions in cells. ${ }^{[9]}$ High levels of GSH are implicated in many diseases such as diabetes, neurodegenerative disorders and cancer. The redox environment in cancer cells is controlled by the NADPH (NADPH, NADP ${ }^{+}$) and GSH (GSH, GSSG) redox systems. ${ }^{[10]}$ In normal cells, the intracellular concentration of GSH has been reported to be $10 \mathrm{mM}$, while the extracellular concentration ranged from 2 to $20 \mu \mathrm{M} .{ }^{[11]}$ However, previous reports found a four-fold increase in GSH concentration in cancer cells, compared to normal cells and even higher levels were found in multi-resistant tumour cells. ${ }^{[12]}$ These findings inspired the development of stimuli-responsive systems targeting high GSH levels. ${ }^{[13]}$ Recently, Akhtar et al. developed water soluble GSH-responsive anion transporters with potential activity to treat cystic fibrosis. ${ }^{[14]}$ However, GSH content in cystic fibrosis cells is lower than in normal cells presumably due to the abnormal extracellular GSH transport ability of CFTR proteins. ${ }^{[14-}$ 15] Talukdar has recently reported GSH switchable synthetic ion channels capable of achieving transmembrane $\mathrm{M}^{+} / \mathrm{Cl}^{-}$symport in presence of elevated levels of GSH. ${ }^{[16]}$ These systems induced caspase-dependant apoptosis in MCF-7 cells which was correlated with the resulting cytotoxicity in rat insulinoma cells (INS-1E), which have high levels of intracellular GSH. ${ }^{[16]}$ Inspired by the high affinity of thiols, such as GSH, for gold as exemplified by Che's seminal work on switch-on fluorescent probes $^{[17]}$ and the reported anion transport activity of 1,3bis(benzimidazol-2-yl)benzenes ${ }^{[18]}$ and similar motifs ${ }^{[19]}$, we adapted Che's systems and designed and synthesized new switchable cycloaurated putative anion transporters based on the structurally related 1,3-bis(benzimidazol-2-yl)pyrimidine (BisBzlmPy). 


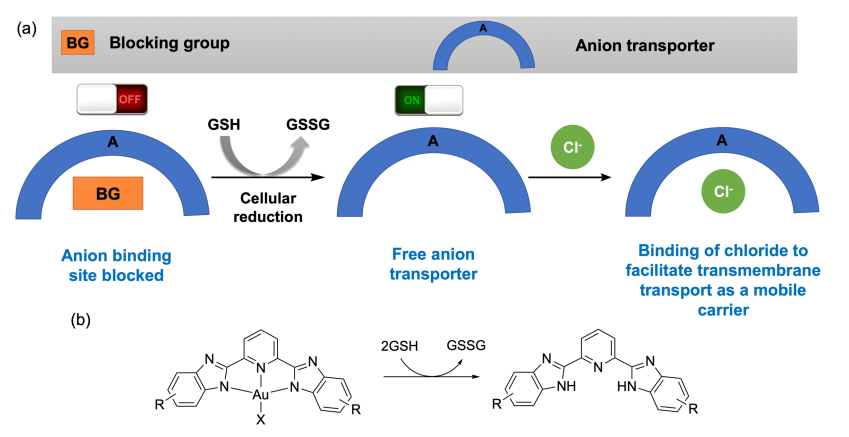

Figure 1: (a) Schematic representation showing the design of switchable anion transporters; (b) reported GSH mediated activation.

Blocking groups, such as gold chloride or gold $\mathrm{N}$-heterocyclic carbene were used to afford the switchable complexes (Figure 1). ${ }^{[13 e, 18]}$ These complexes were designed to be reduced by GSH as a stimulus to liberate the anion transporters that can bind chloride and facilitate transmembrane transport (Figure 1).

\section{Results and Discussion}

Unsubstituted BisBzImPy 1 was synthesized by condensation of o-phenylenediamine with dipicolinic acid in phosphoric acid at $200{ }^{\circ} \mathrm{C}$ for $6 \mathrm{~h}$ (Figure 2a, Scheme S1). ${ }^{[20]}$ Attempts to synthesize ditrifluoromethyl BisBzlmPy derivative 2 using the same procedure failed. BisBzImPy derivatives 2-4 were obtained in 42$54 \%$ yields by adopting an alternative procedure using sodium metabisulfite and 2,6-pyridinedicarboxaldehyde (Scheme S2). ${ }^{[21]}$ Dinitro BisBzImPy derivative $\mathbf{5}$ was prepared by nitration of $\mathbf{1}$ and showed low solubility in most solvents. ${ }^{[20 b]}$ The structure of 2 was confirmed by X-ray diffraction analysis (Figure 2d). Slow evaporation of DMSO solution of $\mathbf{2}$ produced single crystals suitable for X-ray diffraction studies. The structure was elucidated and showed the formation of a 1:1 complex between transporter 2 and DMSO via two hydrogen bonds with nitrogen atoms of benzimidazole $(\mathrm{NH} \cdots \mathrm{O}$ distances of 1.991 (12) $\AA$ and 2.018(12) $\AA$ and $\mathrm{NH} \cdots \mathrm{O}$ bond angles of $169.21(11)^{\circ}$ and $170.77(10)^{\circ}$ (Figure 2d).

Gold chloride derivatives 6 and 7 were readily prepared by heating at reflux BisBzImPy derivatives 2 and 3, respectively, with $\mathrm{KAuCl}_{4}$ and anhydrous sodium acetate in methanol for $3 \mathrm{~h}$ (Figure $2 b$, Scheme S3). ${ }^{[17]}$ We were able to obtain single crystals of complex 7 (Figure 2e, S45) by slow evaporation of acetone solution of $\mathbf{7}$. The crystal structure of $\mathbf{7}$ showed a heptacyclic flat planar gold chloride complex. The bond lengths between Au(III) and nitrogen atoms of benzimidazole and pyridine rings were 1.98(2)-1.99(2) $\AA$. The angle between the nitrogen atom of pyridine, $\mathrm{Au}(\mathrm{III})$ and nitrogen atoms of benzimidazole (N-Au-N) were $79.24(9)^{\circ}$ and $80.23(9)^{\circ}$, respectively, while the angle between chloride, Aulll, and nitrogen atoms of benzimidazole were (Cl-Au-N) $100.02(7)^{\circ}$ and $100.45(7)^{\circ}$, respectively. 1-Butyl3-methyl imidazolium bromide 10 and $\mathrm{AgBr} N$-heterocyclic carbene (NHC) derivative $\mathbf{1 1}$ were prepared according to literature procedures. ${ }^{[22]}$ Reaction of 6 and 7 with NHC 11, silver triflate in acetonitrile in the dark gave complexes 8 and $\mathbf{9}$ in $13 \%$ and $12 \%$ yields, respectively. ${ }^{[17]}$

A single crystals of the $\mathrm{Au}(\mathrm{III})$ complex 8 and 9 were obtained by vapour diffusion of $\mathrm{Et}_{2} \mathrm{O}$ into a $\mathrm{CH}_{3} \mathrm{CN}$ solution of 8 and an acetone solution of $\mathbf{9}$, respectively. The X-ray crystal structures of 8 and 9 revealed that the NHC plane is almost perpendicular to the BizBzlmPy plane with torsion angles of $73.79(5)^{\circ}$ and $81.23(4)^{\circ}$ for complexes 8 and $\mathbf{9}$, respectively (Figures 2f, S46 and S47). The bond lengths between $\mathrm{Au}(\mathrm{III})$ and nitrogen atoms of benzimidazole and pyridine rings were 2.007(5)-2.015(4) $\AA$ for complex 8 and 1.988(4)-2.009(4) $\AA$ for complex 9, slightly longer than in complex 7 . (a)

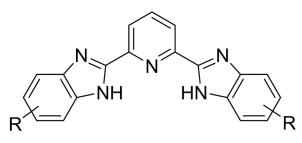

$1(R=H), 2\left(R=5(6)-C_{3}\right), 3\left(R=4,6-B_{i s C F}\right)$ $4(\mathrm{R}=5,6$-Difluoro $), 5\left(\mathrm{R}=5-\mathrm{NO}_{2}\right)$

(b)

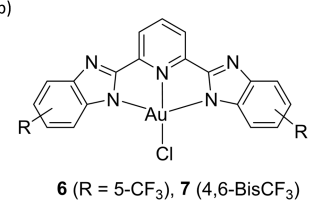

(c)

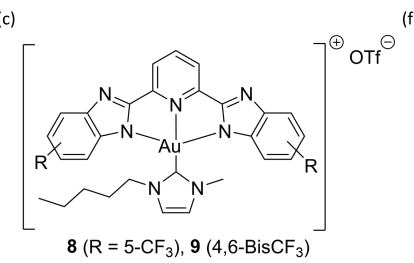

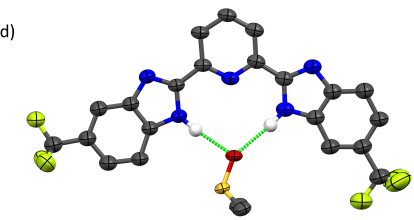

(e)
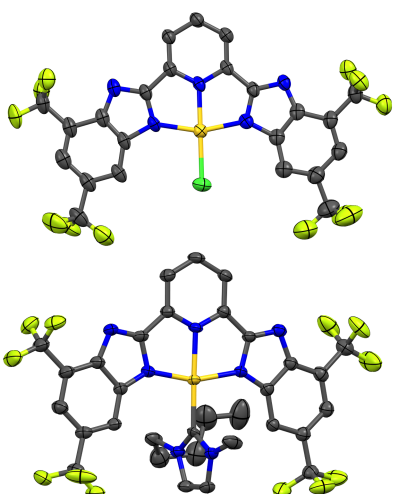

Figure 2: (a) Structure of $O N$ anion transporters 1-5. (b) and (c) Structure of OFF anion transporters 6-9 tested in this paper. (d) ORTEP diagram of anion transporter 2. DMSO complex. (e) Crystal Structures of 7. (f) ORTEP diagram of 9 (trifluoromethylsulfonate was removed for clarity). Thermal ellipsoids are at the $50 \%$ probability level.

To gain a better understanding of the anionophoric properties of the compounds, NMR chloride binding studies were performed in DMSO- $d_{6} / 0.5 \% \mathrm{H}_{2} \mathrm{O}$ with TBACl. Data were fitted globally to the $1: 1$ binding model for transporters $\mathbf{1 - 4}$, while transporter $\mathbf{5}$ was omitted from this study due to it is low solubility (Table 1, Figures S90-93). Anionophores 1-4 demonstrate moderate chloride affinities with $K_{\mathrm{a}}$ ranging from 63 to $132 \mathrm{M}^{-1}$ under the competitive solvent conditions.

Table 1 Lipophilicity, chloride binding and membrane transport properties of compounds 1-5.

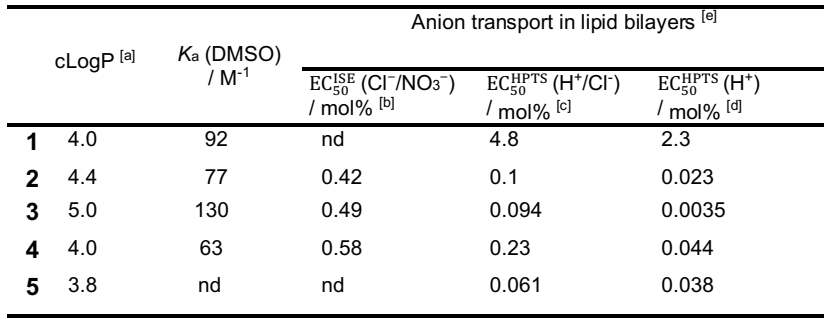

[a] clog $\mathrm{P}$ values calculated using VCCLab. [b] $\mathrm{EC}_{50}$ from the $\mathrm{Cl} / \mathrm{NO}_{3}$ exchange assay. [c] EC50 from the $\mathrm{KCl}$ assay measuring $\mathrm{H}^{+} / \mathrm{Cl}^{-}$symport. [d] $\mathrm{EC}_{50}$ from the $\mathrm{KGlu}$ assay measuring $\mathrm{H}^{+}$uniport. nd: not determined for solubility reasons.

A single crystal of anionophore 2 with chloride was obtained for X-ray diffraction by vapour diffusion of $\mathrm{Et}_{2} \mathrm{O}$ into a $\mathrm{CH}_{3} \mathrm{CN}$ solution of transporter 2 and TEACl (Figure 3). The structure revealed the formation of two hydrogen bonds from the imidazole $\mathrm{NHs}$ to chloride, with $\mathrm{NH}^{\cdots} \mathrm{Cl}^{-}$distances of 2.41(9) and 2.63(8) $\AA$. The $\mathrm{Cl}^{-}$ion was additionally stabilised by a hydrogen bond with a water molecule with an $\mathrm{OH}^{\cdots} \mathrm{Cl}^{-}$distance 2.29(6) $\AA$ (Figure 3). 
This water molecule connects the chloride anion and the second receptor molecule by acting as a hydrogen bond acceptor to the imidazole NHs, with distances 2.13(7) and 2.25(8) $\AA$. The assembly is further stabilized with a hydrogen-bonded water dimer (Figure 3).

(a)

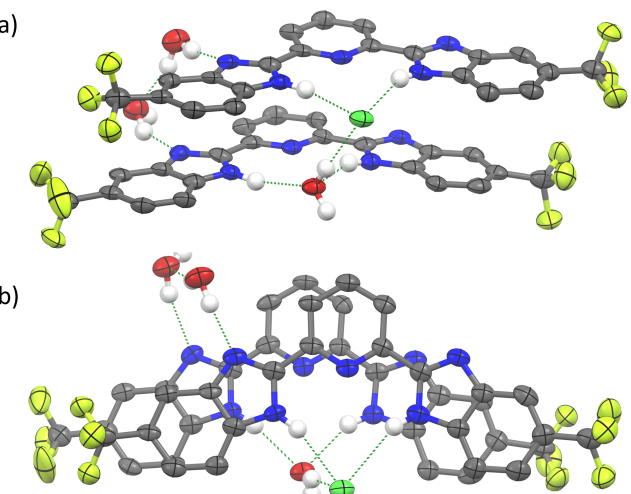

Figure 3: Side (a) and top (b) views of crystal structure of $2 . \mathrm{Cl}^{-}$complex. Counterions $\left(\mathrm{TEA}^{+}\right)$and some solvent molecules have been omitted for clarity. Thermal ellipsoids are at the $50 \%$ probability level. See also Figure S22 for complete structures.

Transporters 1-5 were investigated for their chloride transport properties via a $\mathrm{Cl}^{-} / \mathrm{NO}_{3}{ }^{-}$exchange assay using a chloride ion selective electrode (ISE) (Figure 4a-e, Table 1). Briefly, 1palmitoyl-2-oleoyl-sn-glycero-3-phosphocholine (POPC) unilamellar liposomes with a diameter of $200 \mathrm{~nm}$ were prepared and loaded with $489 \mathrm{mM} \mathrm{KCl}$, buffered at $\mathrm{pH} 7.2$ and suspended in $489 \mathrm{Mm} \mathrm{KNO}_{3}$ solution buffered at $\mathrm{pH}$ 7.2. The chloride efflux due to $\mathrm{Cl}^{-} / \mathrm{NO}_{3}{ }^{-}$exchange was monitored using chloride ion selective electrode upon addition of DMSO solution of transporters 1-5 to the prepared liposomes. ${ }^{[23]}$ At the same concentration, compounds 1-5 showed better transport properties when transporter loading volume increased from $10 \mu \mathrm{L}$ to $40 \mu \mathrm{L}$ (Figures S77-80), presumably due to better deliverability in a higher volume of DMSO. Chloride efflux at different concentrations of tested compounds (expressed as mol\% with respect to lipid concentration (rtl)) was measured to obtain Hill plots and to calculate an $\mathrm{EC}_{50}$ value (defined as the concentration required to achieve $50 \%$ the chloride efflux at $270 \mathrm{~s}$ ) and a Hill coefficient $(n)$. The Hill coefficient indicates the stoichiometry of the formed complex during the anion transport across the lipid bilayer, while $\mathrm{EC}_{50}$ is used as a measure of anion transporter potency.

Compounds 1 and $\mathbf{5}$ were excluded from Hill plot analysis due to low potency of compound $\mathbf{1}$ and the limited solubility of transporter $\mathbf{5}$. Among tested anionophores 2-4, transporter $\mathbf{2}$ was the most active with $\mathrm{EC}_{50}=0.42 \mathrm{~mol} \%$, followed by transporters 3 and $4\left(E_{50}=0.49\right.$ and $0.58 \mathrm{~mol} \%(\mathrm{rtl})$, respectively) (Figure $4 \mathrm{~d}$, Table 1). A cationophore coupling assay, using valinomycin (VIn) or monensin (Mon) was used to investigate the mechanism of transport facilitated by the anionophores. Such assays are used to determine the activities of $\mathrm{Cl}^{-}$uniport (coupling with valinomycin) and $\mathrm{H}^{+} / \mathrm{Cl}^{-}$symport (coupling with monensin) (Figure $4 \mathrm{e}){ }^{[23]}$ The results of the assays showed that compounds 1-5 were capable of both $\mathrm{Cl}^{-}$uniport and $\mathrm{H}^{+} / \mathrm{Cl}^{-}$symport (Figure $4 \mathrm{e}$ ). Very low $\mathrm{Cl}^{-}$efflux was observed in the absence of cationophores (Figure $4 \mathrm{e}$ ), confirming that $\mathrm{K}^{+} / \mathrm{Cl}^{-}$symport did not significantly contribute to the $\mathrm{Cl}^{-}$transport observed in the $\mathrm{Cl}^{-} / \mathrm{NO}_{3}{ }^{-}$exchange assay.

BisBzImPy derivatives 1-5 were then investigated using 8hydroxypyrene-1,3,6-trisulfonic acid (HPTS) assays (Figure 4fi). ${ }^{[23]}$ The HPTS- $\mathrm{KCl}$ assay (Figure $4 \mathrm{f}$ ) measures the activity of anion transporters in facilitating $\mathrm{H}^{+} / \mathrm{Cl}^{-}$cotransport leading to the dissipation of $\mathrm{pH}$ gradients. Consistent with the results of the ISE assay, the unsubstituted BisBzImPy 1 emerged as the transporter with the lowest activity in $\mathrm{HPTS}-\mathrm{KCl}(\mathrm{HCl}$ symport, Figure $4 \mathrm{j})$ assay with an $\mathrm{EC}_{50}=4.8 \mathrm{~mol} \%$. The addition of electronwithdrawing groups to the benzimidazole rings (2-5) increased the ability of the compounds to facilitate $\mathrm{H}^{+} / \mathrm{Cl}^{-}$symport, with $\mathrm{EC}_{50}$ values of these compounds ranging from $0.23 \mathrm{~mol} \%$ to 0.061 mol\%, (Figure 4j, Table 1). The activity decreased in the order of $\mathbf{5}>\mathbf{3}>\mathbf{2}>\mathbf{4}$. It should be noted that the HPTS assay is more sensitive than the ISE assay because of the low $\mathrm{Cl}^{-}$efflux required ( $5 \mathrm{mM}$ compared with $489 \mathrm{mM}$ in the ISE assay). This has led to identification of $\mathbf{5}$ as the most active transporter despite its low deliverability limiting its activity at high concentrations as observed in the ISE assay. To further investigate the intrinsic ability of transporters $\mathbf{1 - 5}$ to transport only chloride, the same assay was employed at $1 \mathrm{~mol} \%$ transporter (mol\% relative to lipid concentration, rtl) and using the protonophore carbonyl cyanide $m$-chlorophenyl hydrazone (CCCP) as an efficient proton transporter. If $\mathrm{H}^{+}$transport is the rate determining step of $\mathrm{HCl}$ symport, CCCP will improve transport and accelerate the $\mathrm{pH}$ dissipation (Figure 4k, S54-58). ${ }^{[23]}$ Anionophores 1-5 demonstrated no CCCP dependence indicating that $\mathrm{H}^{+}$transport by these compounds was not the rate-limiting process in this assay.

We next examined the abilities of transporters 1-5 to transport only protons using a $\mathrm{H}^{+}$transport assay by replacing potassium chloride with potassium gluconate (KGlu) (Figure $4 \mathrm{~h}$ ) and using valinomycin to facilitate $\mathrm{K}^{+}$transport and dissipate the membrane potential generated by $\mathrm{H}^{+}$transport. ${ }^{[23]}$ Gluconate is a relatively large hydrophilic anion that can be considered untransportable ${ }^{[23]}$. Apart from the unsubstituted BisBzImPy $1\left(E_{50}=2.3 \mathrm{~mol} \%\right)$, tested compounds 2-5 showed a remarkable protonophoric activity with $\mathrm{EC}_{50}$ spanning from 0.044 to $0.0035 \mathrm{~mol} \%$, rtl, (Table 1). These values are lower than the $\mathrm{EC}_{50}$ for $\mathrm{H}^{+} / \mathrm{Cl}^{-}$symport, confirming faster $\mathrm{H}^{+}$transport than $\mathrm{Cl}^{-}$transport. The activity of transporters 2-5 as protonophores decreased in the order of 3> $2>\mathbf{5}>\mathbf{4}$ (Table 1). To further investigate the potential interaction between fatty acids and the tested anion transporters 1-5, oleic acid (1 mol\%) or BSA treated liposomes (to remove fatty acids) were used (Figure 4k). It was reported that some transporters could transport $\mathrm{H}^{+}$indirectly by facilitating the transmembrane flipflop of deprotonated fatty acids. ${ }^{[23-24]}$ The transport activity in $\mathrm{KCl}$ and KGlu assays were not affected by addition or removal of fatty acids, which indicate that $\mathrm{H}^{+}$transport by $1-5$ is fatty acidindependent and presumably occurs via deprotonation of the $\mathrm{NH}$ groups. A selectivity assay has demonstrated preferential transport of more lipophilic anions including $\mathrm{Br}^{-}, \mathrm{I}^{-}, \mathrm{NO}_{3}{ }^{-}$, and $\mathrm{ClO}_{4}^{-}$ over $\mathrm{Cl}^{-}$for all anionophores 1-5 (Figures S69-S73). Identical transport rates were found when the $\mathrm{K}^{+}$in the HPTS assay was replaced with $\mathrm{Na}^{+}$(Figures $\mathrm{S74-75}$ ) consistent with the lack of metal ion transport. A liposomal leakage experiment performed using self-quenching calcein confirm the absence of non-specific membrane leakage (Figure S76). ${ }^{[24]}$ 


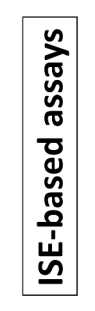

(a)

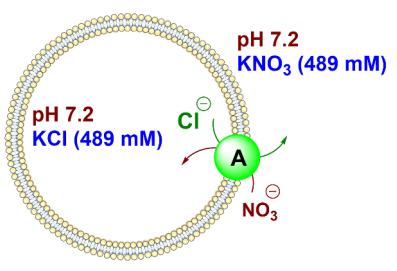

(d)

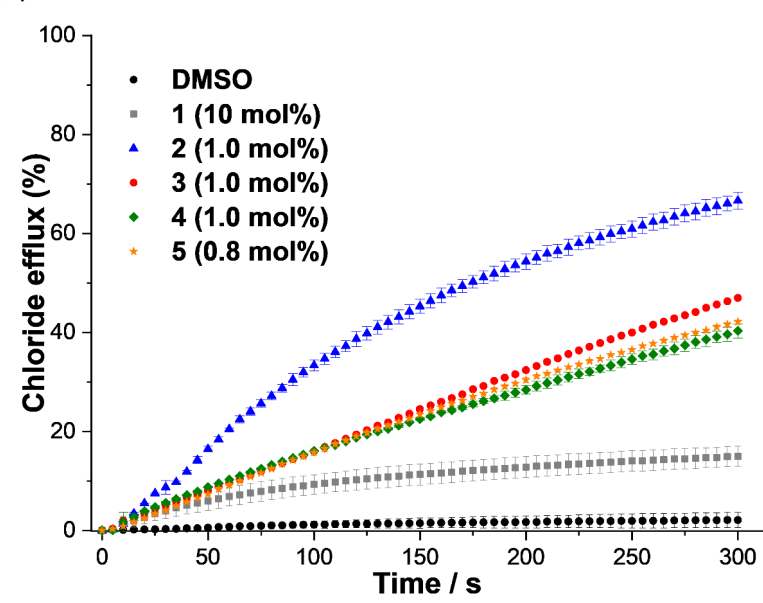

(b)

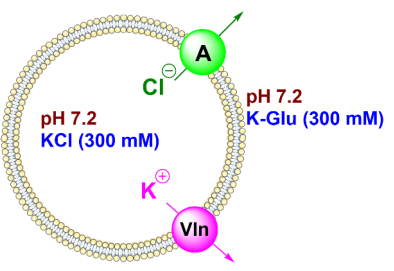

(c)

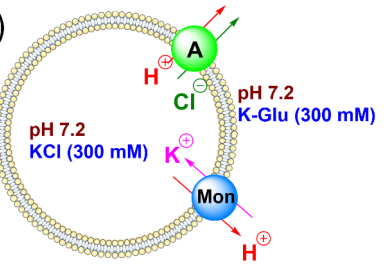

(e)

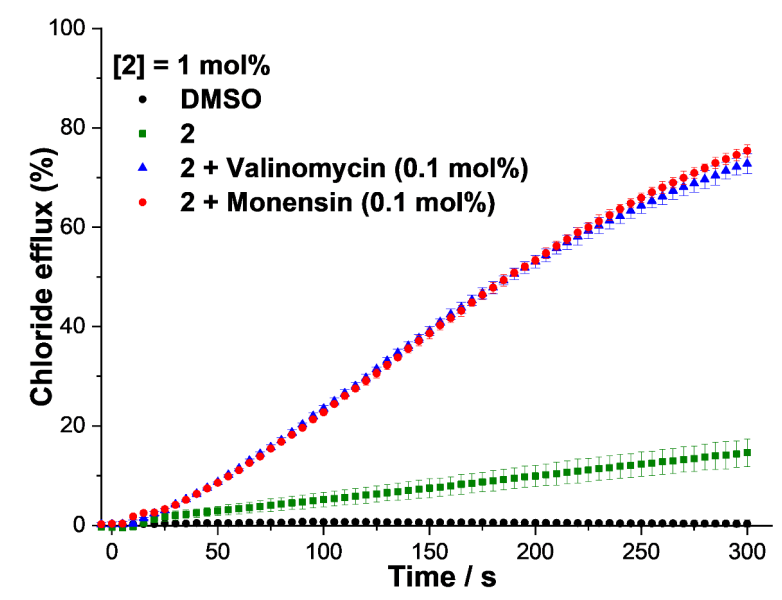

(h)

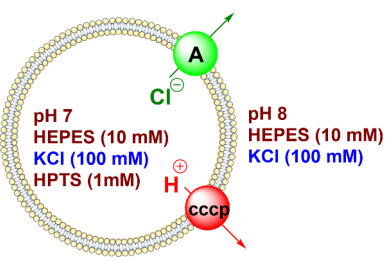

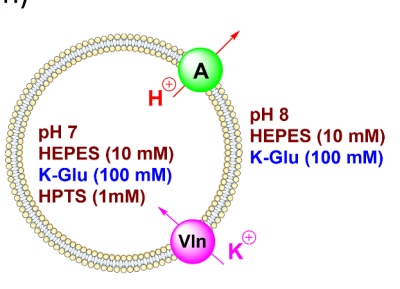

(i) (j)

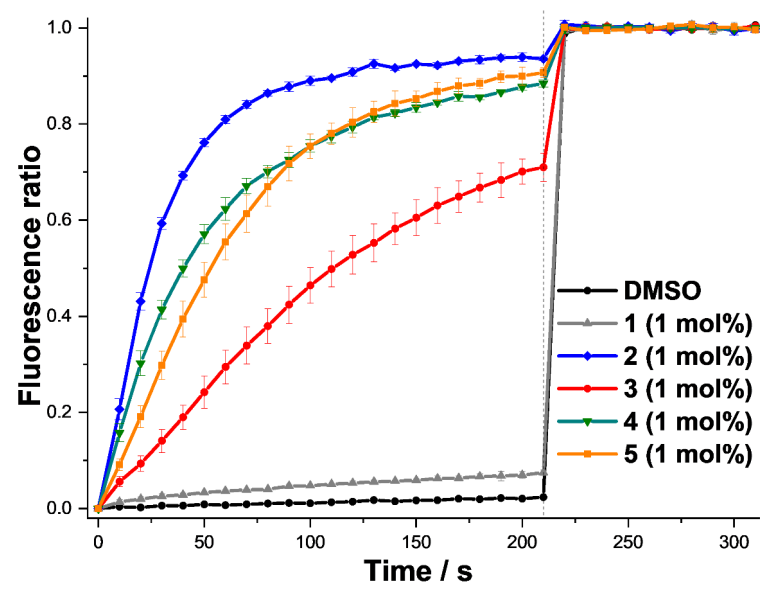

(k)

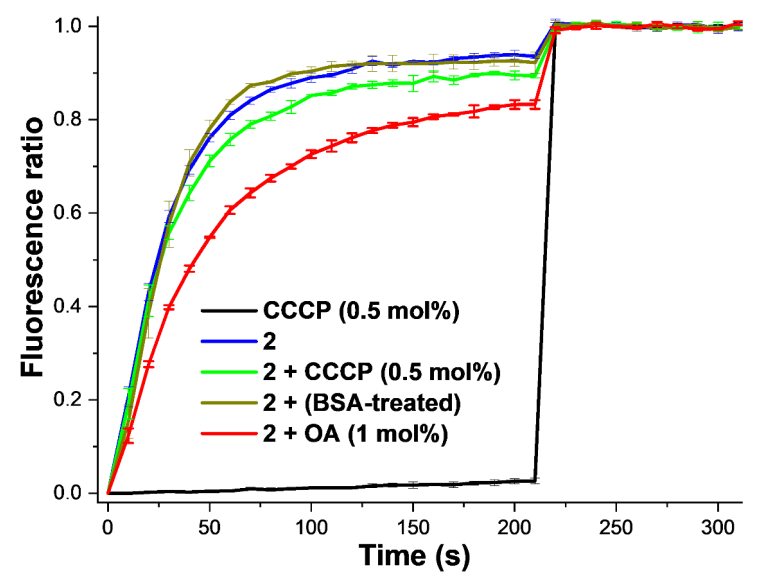

Figure 4: (a-c) Schematic representation of ISE-based assays used to investigate the mechanism of anion transport of receptors 1-5 (a) $\mathrm{Cl}^{-} / \mathrm{NO}_{3}{ }^{-}$antiport, (b) and (c) cationophore coupled- $\mathrm{KCl}$, valinomycin and monensin to measure the $\mathrm{Cl}^{-}$uniport and $\mathrm{M}^{+} / \mathrm{Cl}^{+}$transport, respectively.

(d) Chloride efflux achieved by transporters 1-5 (1.0 mol\% (rtl) for transporters 1-4 and $0.8 \mathrm{~mol} \%$ (rtl) for transporter $\mathbf{5}$ from unilamellar POPC vesicles containing $489 \mathrm{mM} \mathrm{KCl}$ buffered to $\mathrm{pH} 7.2$ with $5 \mathrm{mM}$ potassium phosphate salts, suspended in $489 \mathrm{mM} \mathrm{KNO}_{3}$ buffered to pH 7.2 with $5 \mathrm{mM}$ phosphate salts. (e) Chloride efflux achieved by transporter 2 at $1.0 \mathrm{~mol} \%$ ( $\mathrm{rtl}$ ) in the absence or presence of cationophores (monensin or valinomycin) monitored over a period of 5 min (see SI for more description).

(f-i)Schematic representation of the HPTS-based assays used in the current study (f) $\mathrm{H}^{+} / \mathrm{Cl}^{-}$symport or $\mathrm{OH}^{-} / \mathrm{Cl}^{-}$antiport (g) the presence of CCCP (protonophore) to asses $\mathrm{Cl}^{-}$uniport (h) the presence of valinomycin to measure the proton flux (i) the effect of fatty acid presence as a fuel on the transport. (j) $\mathrm{H}^{+} / \mathrm{Cl}^{-}$symport or $\mathrm{OH}^{-} / \mathrm{Cl}^{-}$antiport facilitated by compounds $\mathbf{1 - 5}(1.0 \mathrm{~mol} \%$ ( $\mathrm{rtl})$ ) from unilamellar POPC vesicles loaded with $100 \mathrm{mM} \mathrm{KCl}$ buffered to $\mathrm{pH} 7.0$ with $10 \mathrm{mM}$ HEPES buffer and $1 \mathrm{mM}$ HPTS internal sensor

(k) Using $\mathrm{KCl}-\mathrm{KOH}$ assay from POPC vesicles loaded with $\mathrm{KCl}(100 \mathrm{mM})$, buffered to $\mathrm{pH} 7.0$ with HEPES (10 mM), different conditions were applied to determine the effect of addition of the protonphore CCCP at $0.5 \mathrm{~mol} \%$ (as a measure of chloride uniport), oleic acid at $1 \mathrm{~mol} \%$ (as a source of fatty acid) and BSA-treated lipid (to test if the transport is fatty acid dependent) on the rate of chloride transport of receptor $2(1 \mathrm{~mol} \%)$. 
We exploited the fluorescence and anion transport properties of transporters $\mathbf{2}$ and $\mathbf{3}$ and examined the corresponding gold complexes 6-9 (complexes 6 and 8 for transporter 2, while complexes $\mathbf{7}$ and $\mathbf{9}$ for transporter $\mathbf{3}$ ) against the effect of the physiological reducing agent GSH and the non-physiological reducing agents DTT and TCEP. Time-dependent reduction kinetics of these complexes were examined under different conditions, including external addition of the reducing agent in an organic solvent (DMSO) and in liposomes (Figure 5, S113-S116). Also, encapsulation of GSH in liposomes was performed to mimic the cellular physiological conditions (Figures S117 and S118). UV-Vis and fluorescence spectroscopies were used to detect the change in the absorbance or fluorescence intensity upon reduction of complexes with GSH, DTT and TCEP. GSH was tested at a molar ratio of 6:1, while TCEP and DTT were used at a molar ratio of $3: 1$, with respect to tested compound. Gratifyingly, fluorescence and absorbance intensities of complexes 6-9 changed over time upon addition of the reducing agents under all

(a)

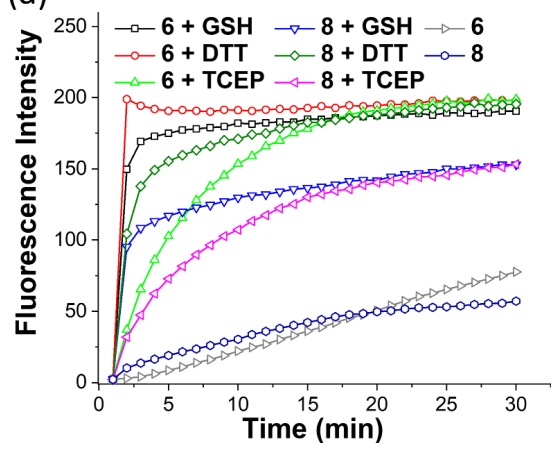

(b)

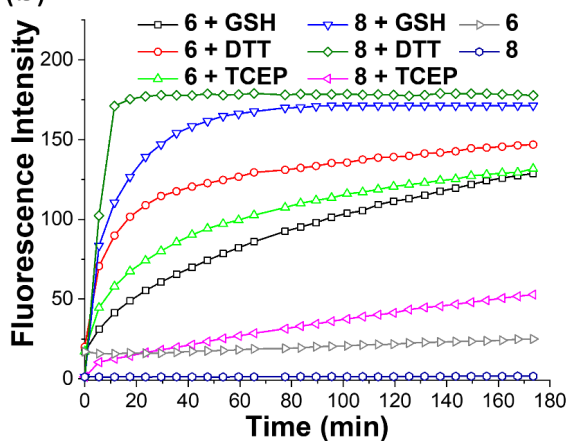

conditions due to redox reactions, but with different rates (Figure $5)$. As indicated from fluorescence and UV-Vis spectra, the reaction between complexes 6-9 and the reducing agent is fast in DMSO, and it took only few seconds to 20 minutes to reach a plateau in both absorbance and fluorescence studies (Figures 5 , S113-115). Moving to the liposome model, with external addition of the reducing agent, the reductions of the gold-NHC complexes 8 and 9 by the tested reducing agents were much faster than their corresponding gold chloride complexes 6 and 7, respectively (Figures 5, S113). DTT emerged as the most powerful reducing agent in liposomes. NHC-gold complexes 8 and $\mathbf{9}$ responded rapidly to DTT with prompt increase in the UV-Vis absorption spectra and reach plateaus after 1-2 minutes. Complex 7 showed a stronger response to DTT than complex 6 with a gradual increase in absorbance and reached a plateau in 30 minutes, while the reduction of complex 6 took $3 \mathrm{~h}$ to reach a plateau.

Figure 5: Reduction of complexes 6-9 as a function of GSH, DTT and TCEP at $25^{\circ} \mathrm{C}$ in (a) DMSO, fluorescence increases due to reduction of $1 \mu \mathrm{M}$ complexes 69 at $6 \mu \mathrm{M}$ of GSH and $3 \mu \mathrm{M}$ of DTT and TCEP over 30 minutes; (b) KCl-Liposomes (SI), fluorescence increases due to reduction of $1 \mu \mathrm{M}$ complexes 6-9 at $6 \mu \mathrm{M}$ of $\mathrm{GSH}$ and $3 \mu \mathrm{M}$ of DTT and TCEP over 3 hours; (c) KCl-Liposomes (SI), absorbance increases due to reduction of $5 \mu \mathrm{M}$ complexes 6-9 at $30 \mu \mathrm{M}$ of GSH and 15 $\mu \mathrm{M}$ of DTT and TCEP over 4 hours. 
The reduction kinetics of compounds $\mathbf{6}$ and 8 by fluorescence spectroscopy showed similar pattern as seen in the UV-Vis studies. Surprisingly, the reduction kinetics of complexes $\mathbf{7}$ and $\mathbf{9}$ could not be followed by fluorescence spectroscopy as the fluorescence intensity of the parent anion transporter $\mathbf{3}$ was diminished in the liposome. GSH and TCEP showed comparable reduction activity towards $A$ (III) chloride complexes 6 and $\mathbf{7}$ and it took less than $3 \mathrm{~h}$ for a complete reduction and a stable fluorescence or absorbance reading (Figure 5). GSH achieved fast reduction of NHC gold complexes $\mathbf{8}$ and $\mathbf{9}$ and reach plateau in 5 minutes as illustrated by UV-Vis spectroscopy. However, compound 8 showed a gradual increase in fluorescence spectra in response to $\mathrm{GSH}$ and took 60 minutes to reach the saturated fluorescence intensity. This may be attributed to encapsulation of the formed free transporter in the membrane bilayer. TCEP demonstrated the slowest reduction for complexes $\mathbf{8}$ and $\mathbf{9}$ and it took more than $3 \mathrm{~h}$ for complex 8 and $1 \mathrm{~h}$ for complex 9 to reach a plateau (Figure 5).

In order to mimic the physiological conditions, the reduction kinetics of complexes 6-9 in GSH-encapsulated liposomes were investigated (Figures S117 and S118). In a similar pattern to the liposome model, complexes 8 and $\mathbf{9}$ showed fast reaction with GSH and reached a plateau in 10 minutes as illustrated from UVVis spectra, while complexes $\mathbf{6}$ and $\mathbf{7}$ showed slower responses than complexes 8 and $\mathbf{9}$. Overall, the redox studies revealed a structural relationship whereby the $\mathrm{NHC}$ gold complexes $\mathbf{8}$ and $\mathbf{9}$ are more labile to reduction than the $\mathrm{Au}$ (III) chloride complexes 6 and 7 . Similarly, bis- $\mathrm{CF}_{3}$ functionalised 7 and 9 demonstrated more rapid redox kinetics as compared to the respective analogous mono- $\mathrm{CF}_{3} \mathbf{6}$ and 8 complexes.

After establishing reduction of $\mathrm{Au}$ (III) complexes and the release of free transporters in liposome membranes, we next investigated the redox switchable transport properties by performing time-dependent transport studies of complexes 6-9 in HPTS- $\mathrm{KCl}$ liposome ( $\mathrm{HCl}$ symport, Figure $4 \mathrm{f}$ and Figure 6 , S119S126) against the three reducing agents. Complexes 6 and 8 (without addition of the reducing agent) showed negligible anion transport activities at $1 \mathrm{~mol} \%$ (Figures 6a and 6c. S119, S120, S123, S124). Interestingly, complexes 7 and 9 showed $35 \%$ and $36 \%$ efflux respectively at $1 \mathrm{~mol} \%$ (rt) (Figure $6 \mathrm{~b}$ and $6 \mathrm{~d}, \mathrm{~S} 121$, S122, S125, S126), indicating that Au (III)-mediated chloride transport across phospholipid membranes. ${ }^{[25]}$

As evidenced by the kinetic studies, DTT is the most potent reducing agent among all tested complexes $6-9$, followed by $\mathrm{GSH}$ and TCEP showed the slowest reduction rate (Figure 6). Importantly, after mixing with the reducing agents for $3 \mathrm{~h}$, complex 6 was "switched on" showing the same chloride efflux as the parent anion transporter 2. Incubation of complex 7 with GSH and TCEP for 30 minutes demonstrated a similar chloride efflux rate to that of the parent anion transporter $\mathbf{3}$, however, incubation with DTT for 30 minutes led to a faster chloride efflux than the parent compound 3 . This is presumably due to anion transport activity of complex 7 itself. In addition, reacting complex 7 with all reducing agents for 3 hours led to a higher chloride efflux than anion transporter 3 at $1 \mathrm{~mol} \%$.

Incubation of complex 8 with DTT or GSH for 5 minutes led to $100 \%$ and $\sim 90 \%$ chloride efflux, respectively, in comparison with the parent transporter 2 , while a longer incubation time of $20 \mathrm{~min}$ led to full restoration of transport activity for both reductants. Incubation of DTT, GSH or TCEP with complex 9 resulted in higher chloride efflux (with GSH and DTT) or similar chloride efflux

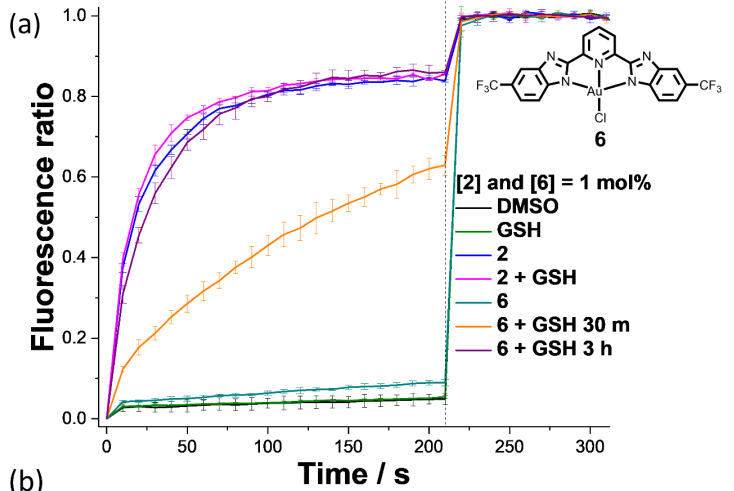

(b)

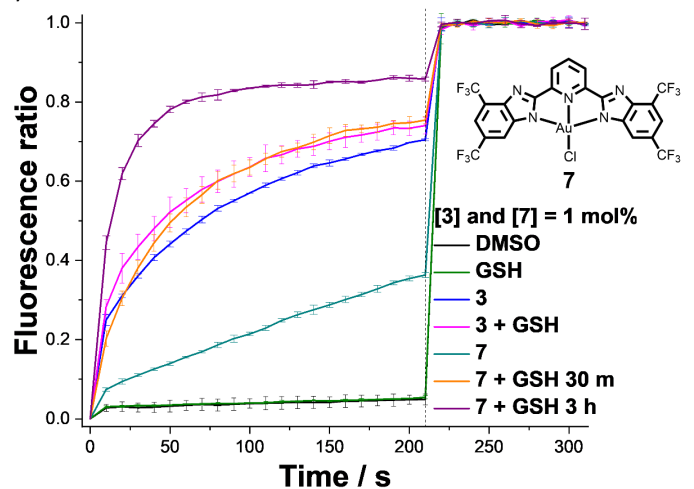

(c)

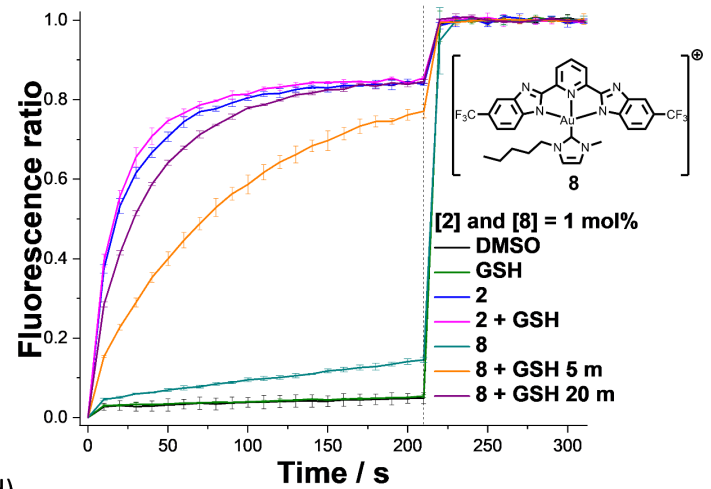

(d)

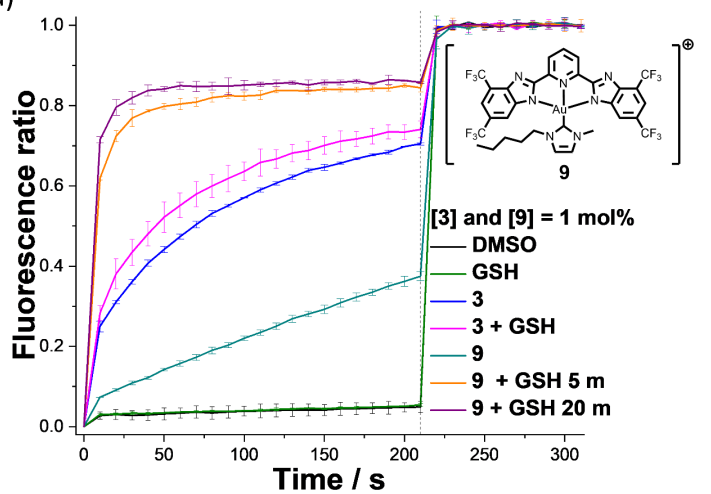

Figure 6. Observed fluorescence ratio response due to $\mathrm{H}^{+} / \mathrm{Cl}^{-}$symport or $\mathrm{Cl}^{-}$ $\mathrm{OH}^{-}$antiport upon reduction of complex 6-9 $(1 \mu \mathrm{M})$ by $\mathrm{GSH}(6 \mu \mathrm{M})$ using $\mathrm{KCl}-$ $\mathrm{KOH}$ assay from POPC vesicles loaded with $\mathrm{KCl}(100 \mathrm{mM})$, buffered to $\mathrm{pH} 7.0$ with HEPES $(10 \mathrm{mM})$ at different time intervals. The test complexes 6-9 (1 $\mathrm{mol} \%$ ) and $\mathrm{KOH}$ were added firstly, then GSH was added at $0 \mathrm{~s}$. DMSO, GSH ( $3 \mathrm{~mol} \%$ ), parent anion transporters 2 and 3 and complex 6-9 (without addition of $\mathrm{GSH}$ ) were used as controls. Detergent was added at $200 \mathrm{~s}$. Ionophore concentrations are shown as ionophore to lipid molar ratios. Error bars represent SD from at least three repeats. 
(with TCEP) in comparison to the parent transporter 3. These time-dependent transport studies correlate to the redox timeline as discussed and the results have demonstrated successful implementation of our binding site blocking strategy to achieve redox-switchable chloride transport.

Preliminary cell viability studies on human embryonic kidney cells (HEK293, non-cancerous), human epithelial mammary cells (MCF10A, non-cancerous) and human colon adenocarcinoma cells (SW620) were investigated in the presence of $5 \mu \mathrm{M}$ of compounds 1-9 (Figure S127). The study demonstrated that the switchable transporters 6-8 are less cytotoxic to normal cell lines HEK293 and MCF10A when compared to their corresponding precursors (2 for complexes $\mathbf{6}$ and $\mathbf{8}, \mathbf{3}$ for complex $\mathbf{7}$ ). This is presumably attributed to the expected lower GSH concentration in normal cell lines, such as MCF10A, when compared to cancerous cells. ${ }^{[26]}$ In SW620 cell line, compounds $\mathbf{7}$ and $\mathbf{8}$ elicited comparable cell viability to parent transporters $\mathbf{3}$ and $\mathbf{2}$, respectively, owing to the switchable nature of these complexes while compound 6 demonstrated a lower cytotoxic effect than its precursor 2 suggesting incomplete switching of this particular complex. Interestingly, complex $\mathbf{9}$ showed higher cytotoxicity in the tested cell lines than the parent transporter $\mathbf{3}$, which may be attributed to the facile switching of $\mathbf{9}$ as demonstrated by the redox switchable transport studies and the release of the gold imidazolium unit. Further biological studies are currently undergoing to study the behaviour of these switchable transporters in cells and to elucidate their mechanism of action.

\section{Conclusion}

We have developed stimuli-responsive gold complexes as efficient switchable anion transporters by complexing bisimidazole anion transporters to $\mathrm{Au}(\mathrm{III})$ to block the anion binding site. The complexes can be reduced to release active anion transporters by reducing agents, including the physiologically predominant GSH. Five BisBzImPy anion transporters 1-5 were synthesized and they have been shown to bind chloride with moderate affinity in DMSO- $d_{6} / 0.5 \% \mathrm{H}_{2} \mathrm{O}$ and facilitate the transport of $\mathrm{H}^{+}, \mathrm{Cl}^{-}$and $\mathrm{NO}_{3}{ }^{-}$in liposomal models. Switchable cycloaurated complexes 6-9 were prepared from two of the most potent anion transporters 2 and 3 . The complexes 6 9 could be reduced to release free bisimidazole anion transporters in both organic solvents and liposomal membranes, with efficiencies of different reducing agents in the order of DTT $>$ GSH $>$ TCEP. Finally, transport studies showed that these switchable anion transporters 6-9 can be switched $\mathrm{ON}$ by all the tested reducing agents to facilitate anion transport. A remarkably rapid reduction of $\mathrm{Au}(\mathrm{III})$ centre was found for complex 9 upon treatment with GSH leading to complete release of the active anion transporter within $5 \mathrm{~min}$. This work provides a new mechanism to switch on anion transport in the presence of the conditions found in disease states such as cancer and may offer a strategy of targeting biologically active transporters in living systems.

\section{Acknowledgements}

PAG thanks the Australian Research Council (DP200100453) and the University of Sydney for funding. MF thanks the University of Wollongong for the University Postgraduate Award and International Postgraduate Tuition Award scholarships and The University of Sydney for an internship.

Keywords: Anion transport • Molecular switch • Gold complexes - Reduction kinetics • supramolecular chemistry.

[1] a) A. Hennig, G. J. Gabriel, G. N. Tew, S. Matile, J. Am. Chem. Soc. 2008, 130, 10338-10344; b) M. Vlatkovic, B. S. Collins, B. L. Feringa, Chem. Eur. J. 2016, 22, 1708017111; c) R. Zhou, S. Zhu, L. Gong, Y. Fu, Z. Gu, Y. Zhao, J. Mater. Chem. B 2019, 7, 2588-2607; d) S. J. Wezenberg, B. L. Feringa, Nat. Commun. 2018, 9, 1984.

[2] N. V. Rao, H. Ko, J. Lee, J. H. Park, Front. Bioeng. Biotech. 2018, 6, 110.

[3] a) S. Lee, A. H. Flood, J. Phys. Org. Chem. 2013, 26, 7986; b) E. N. Howe, N. Busschaert, X. Wu, S. N. Berry, J. Ho, M. E. Light, D. D. Czech, H. A. Klein, J. A. Kitchen, P. A. Gale, J. Am. Chem. Soc. 2016, 138, 8301-8308; c) M. Vlatkovic, B. L. Feringa, S. J. Wezenberg, Angew. Chem. 2016, 128, 1013-1016; Angew. Chem. Int. Ed. 2016, 55 , 1001-1004; d) D. A. McNaughton, X. Fu, W. Lewis, D. M. D'Alessandro, P. A. Gale, Chemistry 2019, 1, 80-88; e) A. Saha, N. Akhtar, V. Kumar, S. Kumar, H. K. Srivastava, S. Kumar, D. Manna, Org. Biomol. Chem. 2019, 17, 57795788.

[4] a) N. H. Evans, P. D. Beer, Angew. Chem. 2014, 126, 11908-11948; Angew. Chem. Int. Ed. 2014, 53, 1171611754; b) N. Busschaert, C. Caltagirone, W. Van Rossom, P. A. Gale, Chem. Rev. 2015, 115, 8038-8155; c) N. Busschaert, S. H. Park, K. H. Baek, Y. P. Choi, J. Park, E. N. W. Howe, J. R. Hiscock, L. E. Karagiannidis, I. Marques, V. Felix, W. Namkung, J. L. Sessler, P. A. Gale, I. Shin, Nat. Chem. 2017, 9, 667-675.

[5] D. C. Gadsby, Nat. Rev. Mol. Cell Biol. 2009, 10, 344-352.

[6] a) F. M. Ashcroft, Ion channels and disease, Academic press, Oxford, 1999; b) A. S. Verkman, L. J. Galietta, Nat. Rev. Drug Disc. 2009, 8, 153-171; c) H. Li, H. Valkenier, L. W. Judd, P. R. Brotherhood, S. Hussain, J. A. Cooper, O. Jurcek, H. A. Sparkes, D. N. Sheppard, A. P. Davis, Nat. Chem. 2016, 8, 24-32.

[7] a) V. Soto-Cerrato, P. Manuel-Manresa, E. Hernando, S. Calabuig-Farinas, A. Martinez-Romero, V. FernandezDuenas, K. Sahlholm, T. Knopfel, M. Garcia-Valverde, A M. Rodilla, E. Jantus-Lewintre, R. Farras, F. Ciruela, R. Perez-Tomas, R. Quesada, J. Am. Chem. Soc. 2015, 137, 15892-15898; b) W. Van Rossom, D. J. Asby, A. Tavassoli, P. A. Gale, Org. Biomol. Chem. 2016, 14, 26452650; c) A. M. Rodilla, L. Korrodi-Gregorio, E. Hernando, P. Manuel-Manresa, R. Quesada, R. Perez-Tomas, V. Soto-Cerrato, Biochem. Pharmacol. 2017, 126, 23-33.

[8] S. H. Park, S. H. Park, E. N. W. Howe, J. Y. Hyun, L. J. Chen, I. Hwang, G. Vargas-Zuniga, N. Busschaert, P. A. Gale, J. L. Sessler, I. Shin, Chem 2019, 5, 2079-2098.

[9] H. J. Forman, H. Zhang, A. Rinna, Mol. Aspects Med. 2009, 30, 1-12.

[10] a) G. Wu, Y. Z. Fang, S. Yang, J. R. Lupton, N. D. Turner, J. Nutr. 2004, 134, 489-492; b) C. Hegedus, K. Kovacs, Z. Polgar, Z. Regdon, E. Szabo, A. Robaszkiewicz, H. J. Forman, A. Martner, L. Virag, Redox Biol. 2018, 16, 59-74.

[11] S. Mura, J. Nicolas, P. Couvreur, Nat. Mater. 2013, 12 , 991-1003.

[12] a) F. Meng, W. E. Hennink, Z. Zhong, Biomaterials 2009, 30, 2180-2198; b) R. Li, Y. Xie, J. Control. Release 2017, 251, 49-67.

[13] a) N. Chan, B. Khorsand, S. Aleksanian, J. K. Oh, Chem. Commun. 2013, 49, 7534-7536; b) L. Jia, D. Cui, J. Bignon, A. Di Cicco, J. Wdzieczak-Bakala, J. Liu, M. H. Li, 
Biomacromolecules 2014, 15, 2206-2217; c) Y. Li, Y. Wu, J. Chen, J. Wan, C. Xiao, J. Guan, X. Song, S. Li, M. Zhang, H. Cui, Nano Lett. 2019, 19, 5806-5817; d) A Sharma, M.-G. Lee, M. Won, S. Koo, J. F. Arambula, J. L. Sessler, S.-G. Chi, J. S. Kim, J. Am. Chem. Soc. 2019, 141, 15611-15618; e) J. L. Hickey, R. A. Ruhayel, P. J.

Barnard, M. V. Baker, S. J. Berners-Price, A. Filipovska, J. Am. Chem. Soc. 2008, 130, 12570-12571.

[14] N. Akhtar, N. Pradhan, A. Saha, V. Kumar, O. Biswas, S. Dey, M. Shah, S. Kumar, D. Manna, Chem. Commun. 2019, 55, 8482-8485.

[15] L. Gao, K. J. Kim, J. R. Yankaskas, H. J. Forman, Am. J. Physiol. Cell Physiol. 1999, 277, L113-118.

[16] J. A. Malla, R. M. Umesh, S. Yousf, S. Mane, S. Sharma M. Lahiri, P. Talukdar, Angew. Chem. 2020, DOI: 10.1002/ange.202000961; Angew. Chem. Int. Ed. 2020, DOI: 10.1002/anie.202000961.

[17] T. Zou, C. T. Lum, S. S. Chui, C. M. Che, Angew. Chem. 2013, 125, 3002-3005; Angew. Chem. Int. Ed. 2013, 52, 2930-2933.

[18] a) C. Montoya, R. Cervantes, J. Tiburcio, Tetrahedron Lett. 2015, 56, 6177-6182; b) C. C. Peng, M. J. Zhang, X. X. Sun, X. J. Cai, Y. Chen, W. H. Chen, Org. Biomol. Chem. 2016, 14, 8232-8236; c) X. H. Yu, X. Q. Hong, W. H. Chen, Org. Biomol. Chem. 2019, 17, 1558-1571.

[19] T. Merckx, C. J. Haynes, L. E. Karagiannidis, H. J. Clarke, K. Holder, A. Kelly, G. J. Tizzard, S. J. Coles, P. Verwilst, P. A. Gale, W. Dehaen, Org. Biomol. Chem. 2015, 13, 1654-1661.

[20] a) A. W. Addison, P. J. Burke, J. Heterocycl. Chem. 1981, 18, 803-805; b) G. Li, J. Huang, M. Zhang, Y. Zhou, D. Zhang, Z. Wu, S. Wang, X. Weng, X. Zhou, G. Yang, Chem. Commun. 2008, 38, 4564-4566.

[21] H. Goeker, M. Alp, Z. Ateş - Alagöz, S. Yıldız, J. Heterocycl. Chem. 2009, 46, 936-948.

[22] a) H. M. Wang, C. Y. Chen, I. J. Lin, Organometallics 1999, 18, 1216-1223; b) E. R. Parnham, R. E. Morris, Chem. Mater. 2006, 18, 4882-4887.

[23] X. Wu, E. N. W. Howe, P. A. Gale, Acc. Chem. Res. 2018 51, 1870-1879.

[24] E. N. W. Howe, P. A. Gale, J. Am. Chem. Soc. 2019, 141, 10654-10660.

[25] D. Milano, B. Benedetti, M. Boccalon, A. Brugnara, E. lengo, P. Tecilla, Chem. Commun. 2014, 50, 9157-9160.

[26] a) A. D. Kim, R. Zhang, X. Han, K. A. Kang, M. J. Piao, Y. H. Maeng, W. Y. Chang, J. W. Hyun, Mol. Med. Rep. 2015, 12, 4314-4319; b) S. H. Cheng, Y. M. Tseng, S. H. Wu, S. M. Tsai, L. Y. Tsai, Sci. Rep. 2017, 7, 15976. 


\section{Entry for the Table of Contents}

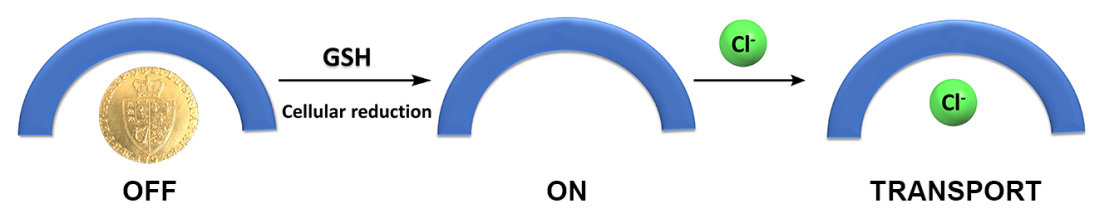

Bis-imidazole-based anion transporters have been complexed to $\mathrm{Au}$ (III) to switch off their anion transport properties. In the presence of reducing agents such as GSH the gold is sequestered from the transporter and transport switched on. This provides a method of targeting anion transporters to tissue with higher concentrations of GSH including tumours.

Institute and/or researcher Twitter usernames: @SydneyChemistry @philgale 


\section{Supporting Information \\ Stimuli-responsive cycloaurated 'OFF-ON' switchable anion transporters}

Mohamed Fares, Xin Wu Deepthi Ramesh, William Lewis, Paul A. Keller, Ethan N.W. Howe, ${ }^{*}$ Ricardo Pérez-Tomás* and Philip A. Gale*

\section{Table of Contents}

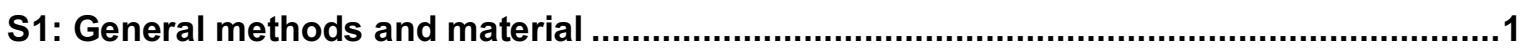

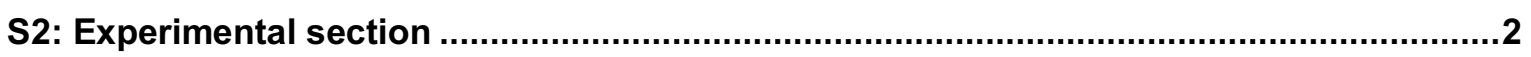

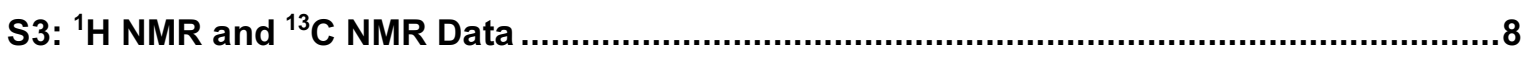

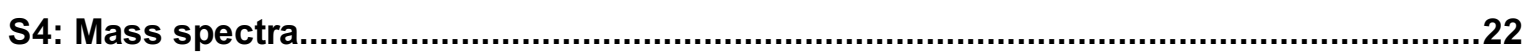

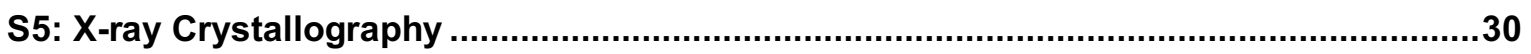

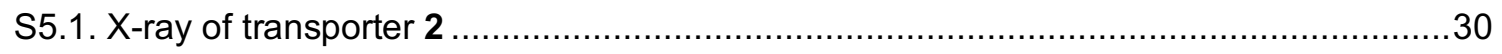

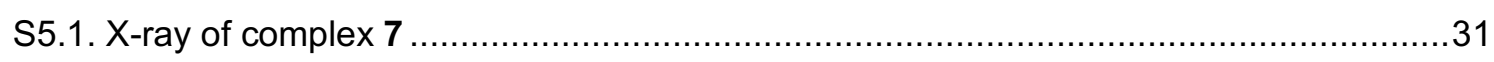

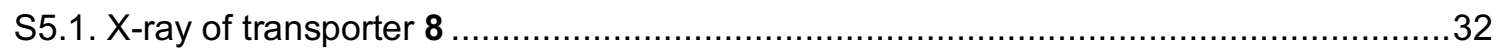

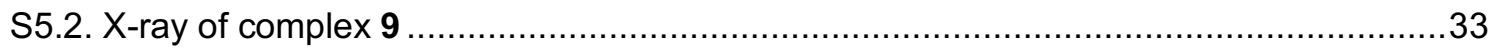

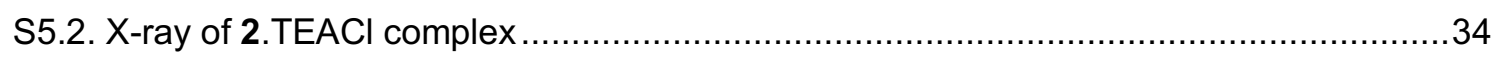

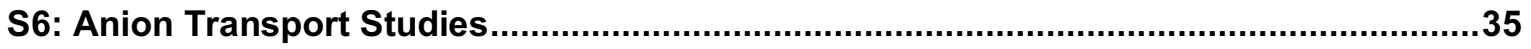

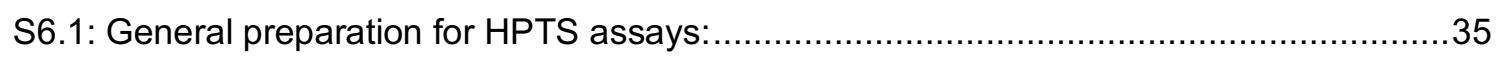

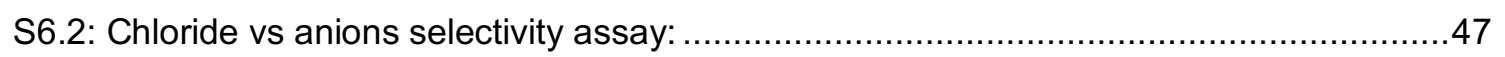

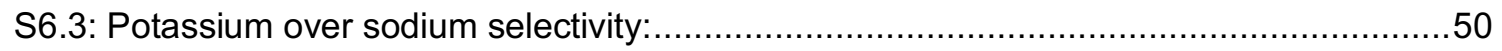

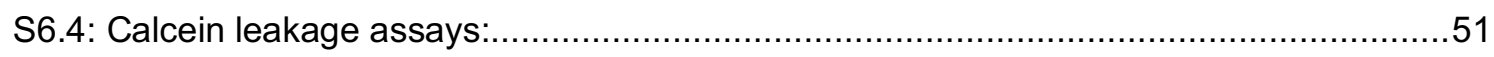

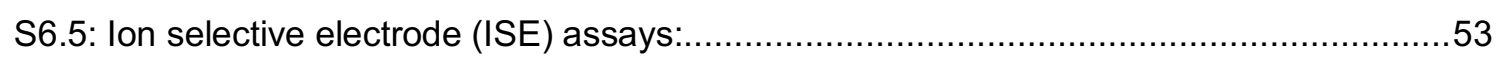

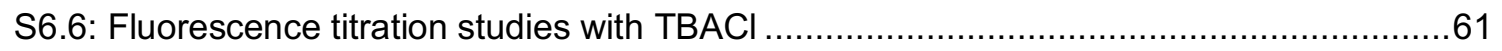

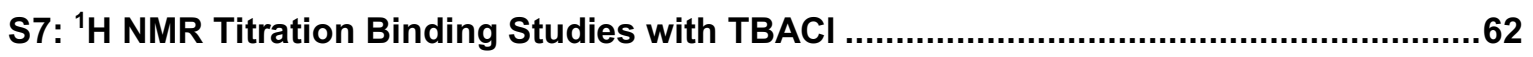

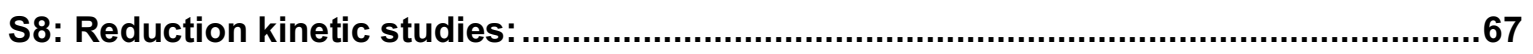

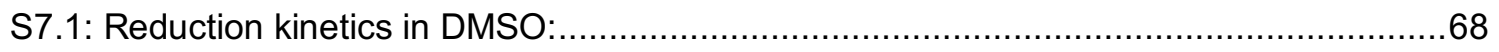

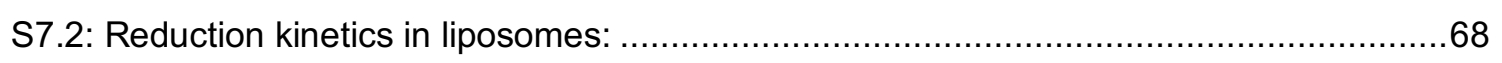

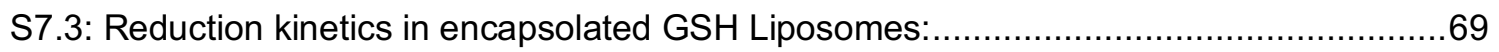

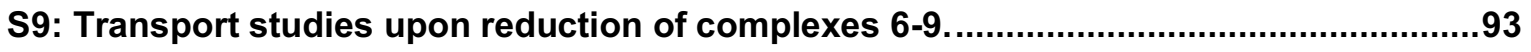

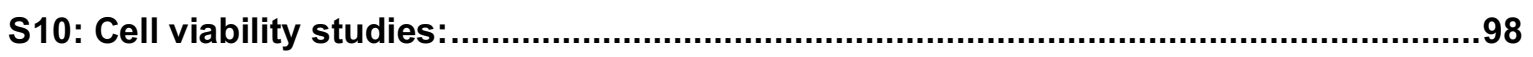

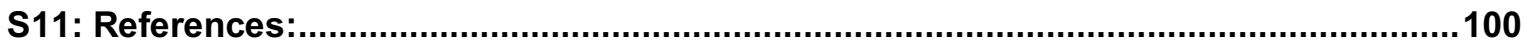




\section{S1: General methods and material}

All reagents and solvents were purified and dried by standard techniques. Reactions were monitored by TLC analysis using silica gel GF/UV 254. NMR spectra were recorded on Bruker 400 MHz FT-NMR spectrometer and Varian Gemini-300BB 300 MHz FT-NMR spectrometers (Varian Inc., Palo Alto, CA). ${ }^{1} \mathrm{H}$ spectra were run at 300 and $400 \mathrm{MHz}$ and ${ }^{13} \mathrm{C}$ spectra were run at 75 and $101 \mathrm{MHz}$, in the stated solvent. Chemical shifts $\left(\delta_{H}\right)$ are reported relative to TMS as internal standard and coupling constant $(J)$ values are reported in Hertz. The abbreviations used are as follows: s, singlet; d, doublet; t, triplet; $\mathrm{m}$, multiplet. Electrospray (ESI single quadrupole) mass spectra have their ion mass to charge values $(\mathrm{m} / \mathrm{z})$ stated with their relative abundances as a percentage in parentheses. Peaks assigned to the molecular ion are denoted as $[\mathrm{M}+\mathrm{H}]$ or $[\mathrm{M}+\mathrm{Na}]$. Column chromatography was performed using silica gel $60(0.063-0.200 \mathrm{~mm})$. Low resolution mass spectra (LRMS) and high resolution mass spectra (HRMS) were recorded using positive/negative ion electrospray ionization (ESI) and atmospheric pressure photoionization (APPI) on Bruker amaZon SL mass spectrometer and Bruker Solarix 2xR 7T Fourier transform ion cyclotron resonance mass spectrometer (FTICR). UV-vis kinetic studies were performed on an Agilent Cary 4000 UV-vis absorption spectrometer equipped with a magnetic stirrer and a temperature controller. Synthesis of 2,6-bis(1H-benzo[d]imidazol-2-yl)pyridine $\quad$ 1, 2,6-bis(5-nitro- $1 H$-benzo[d]imidazol-2yl)pyridine 5, 1-butyl-3-methyl imidazolium bromide 10 and $\mathrm{Ag} \mathrm{N}$-hetercyclic carbene $(\mathrm{NHC})$ derivatives 11 were attempted according to literature. ${ }^{[1]}$ 


\section{S2: Experimental section}

1- Synthesis of anion transporter $1^{[1 a, 2]}$<smiles>Nc1ccccc1N</smiles>

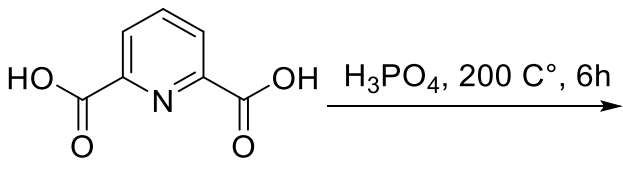<smiles>c1cc(-c2nc3ccccc3[nH]2)nc(-c2nc3ccccc3[nH]2)c1</smiles>

Scheme S1: Synthesis of 1

Dipicolinic acid (pyridine-2,6-dicarboxylic acid) $(1.67 \mathrm{~g}, 10 \mathrm{mmol})$ and 0 phenylenediamine $(2.38,22 \mathrm{mmol})$ were suspended in orthophosphoric acid $(30 \mathrm{~mL})$ at $200{ }^{\circ} \mathrm{C}$ for $6 \mathrm{~h}$. After cooling, the reaction melt was poured in $500 \mathrm{~mL}$ of cold water and vigorous stirring was applied. The blue-green precipitate was filtered out and then stirred with hot $10 \%$ aqueous sodium carbonate for $10 \mathrm{~min}$. The formed solid was collected by filtration and crystalized from methanol to give 1 as a white powder $(1.35 \mathrm{~g}, 43 \%)$.

Characterisation of 2,6-bis $(1 \mathrm{H}$-benzo[d]imidazol-2-yl)pyridine 1:

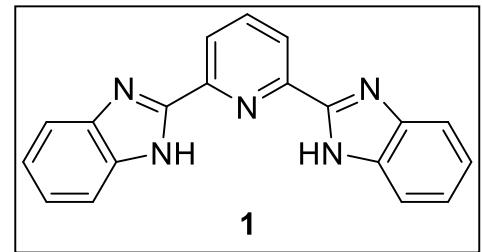

${ }^{1} \mathrm{H}$ NMR (400 MHz, DMSO, $\left.\boldsymbol{d}_{6}\right)$ ס: 7.23-7.36 (m, 4H, ArH), 7.77 (dd, $J=8.0,24 \mathrm{~Hz}, 4 \mathrm{H}, \mathrm{Ar}-\mathrm{H}), 8.18$ (t, $J=8.0 \mathrm{~Hz}, 1 \mathrm{H}$, $\operatorname{Ar}-\mathrm{H}), 8.36(\mathrm{~d}, J=8.0 \mathrm{~Hz}, 2 \mathrm{H}, \mathrm{Ar}-\mathrm{H}), 13.10$ (br s, 2H, $2 \mathrm{x}-$ $\mathrm{NH}) ;{ }^{13} \mathrm{C}$ NMR (101 MHz, DMSO, $\left.\boldsymbol{d}_{6}\right) \mathbf{\delta}: 112.3,120.2$, 121.8, 122.7, 124.2, 134.9, 139.7, 144.6, 148.3, 151.0. HRMS (ESI ${ }^{+}$) calcd for $\mathrm{C}_{19} \mathrm{H}_{13} \mathrm{~N}_{5} \mathrm{Na}(\mathrm{M}+\mathrm{Na}): \mathrm{m} / \mathrm{z} 334.10632$, found 334.10608 (0.70 ppm).

\section{2- General procedure A of anion transporters 2-4 ${ }^{[3]}$}

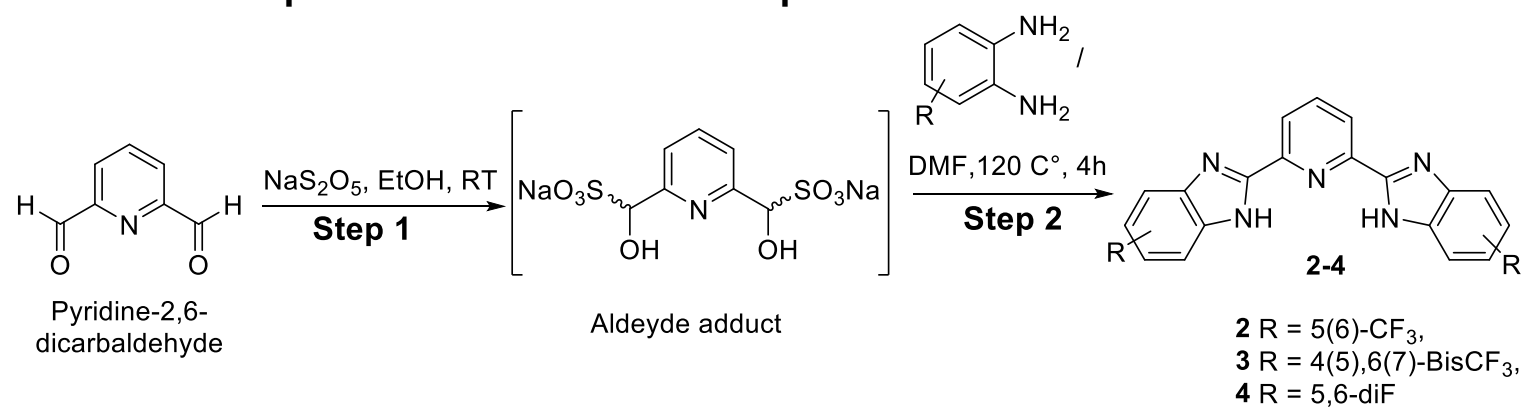

Scheme S2: Synthesis of 2-4 
Step 1. To a suspension of pyridine-2,6-dicarbaldehyde (270 $\mathrm{mg}, 2 \mathrm{mmol}$ ) in ethanol (7 $\mathrm{mL})$, was slowly added a solution of sodium metabisulfite $(213 \mathrm{mg}, 1.1 \mathrm{mmol})$ in water $(1 \mathrm{~mL})$. The mixture was stirred vigorously at room temperature and ethanol $(10 \mathrm{~mL})$ was added. The reaction flask was left overnight at $0{ }^{\circ} \mathrm{C}$ and the resulting pink precipitate was filtered and dried to give the aldehyde adduct $(611 \mathrm{mg}, 89 \%)$ as a pink powder. The aldehyde adduct was used without further purification.

\section{Step 2.}

A mixture of aldehyde adducts $(342 \mathrm{mg}, 1 \mathrm{mmol}$ ) and the appropriate phenylene diamine (2 mmol) in dry DMF (1mL) was heated at $120{ }^{\circ} \mathrm{C}$ for $4 \mathrm{~h}$. The mixture was cooled overnight and poured on brine solution and left to stir overnight. The resulting solid filtered, washed with water $(3 \times 10 \mathrm{~mL})$, dried and recrystallized from ethanol to give anion transporter 2-4.

Characterisation of 2,6-bis(5-(trifluoromethyl)-1H-benzo[d] imidazol-2-yl)pyridine 2

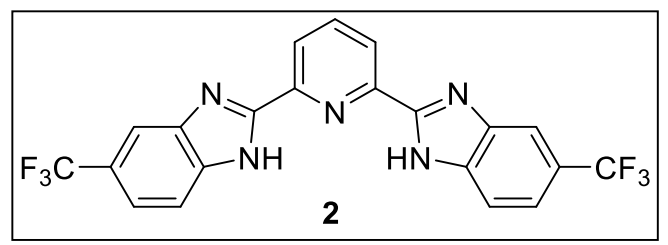

Using general procedure $\mathbf{A}$ and 4(trifluoromethyl)benzene-1,2-diamine (352 mg, 2 mmole), anion transporter 2 was obtained as a white solid (482 mg, 54\%). ${ }^{1} \mathrm{H}$ NMR (400 $\mathrm{MHz}$,

$\mathrm{CD}_{3} \mathrm{CN}$ ) $\delta: 7.39$ (dd, J = 1.0, 9.0 Hz, 2H, Ar-H), 7.54 (d, J = 8.0 Hz, 2H, Ar-H), 7.72 (t, J $=8.0 \mathrm{~Hz}, 1 \mathrm{H}, \mathrm{Ar}-\mathrm{H}), 7.77-7.83(\mathrm{~m}, 2 \mathrm{H}, \mathrm{ArH}), 8.02(\mathrm{~d}, \mathrm{~J}=8 \mathrm{~Hz}, 2 \mathrm{H}, \mathrm{Ar}-\mathrm{H}) ;{ }^{13} \mathrm{C}$ NMR $(101$ $\mathrm{MHz}, \mathrm{CD}_{3} \mathrm{CN}$ ) $\delta: 118.1,120.6,121.9,123.1,124.6,125.0\left(\mathrm{~d}, J^{2} \mathrm{C}-\mathrm{F}=31 \mathrm{~Hz}\right), 127.3,130.0$, 139.5, 147.9, 153.2. HRMS (ESI+) calcd for $\mathrm{C}_{21} \mathrm{H}_{11} \mathrm{~F}_{6} \mathrm{~N}_{5} \mathrm{Na}(\mathrm{M}+\mathrm{Na}): \mathrm{m} / \mathrm{z}$ 470.08109, found 470.08088 (0.49 ppm).

\section{Characterisation of 2,6-bis(4,6-bis(trifluoromethyl)-1 $\mathrm{H}$-benzo[d]imidazol-2-yl)pyridine 3}

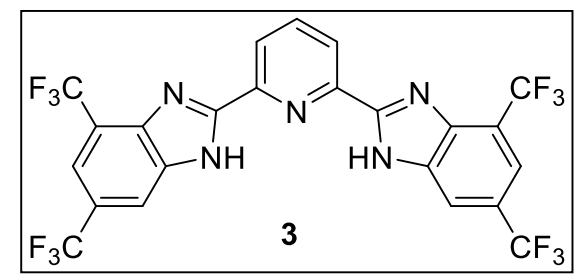

Using general procedure $\mathbf{A}$ and 4(trifluoromethyl)benzene-1,2-diamine (488 mg, 2 mmole), anion transporter 3 was obtained as a white solid (495 mg, 42\%). ${ }^{1} \mathrm{H}$ NMR (300 Hz, $\mathrm{CD}_{3} \mathrm{CN}$ ) $\delta$ : 7.78-7.90 (m, 2H, ArH), $8.13(\mathrm{t}, J=8.0 \mathrm{~Hz}, 1 \mathrm{H}, \mathrm{Ar}-\mathrm{H})$, 8.18-8.25 (m, 2H, ArH), 8.40 (d, J = 8.0 Hz, 2H, Ar-H); $\left.{ }^{13} \mathrm{C} \mathrm{NMR} \mathrm{(126} \mathrm{Hz,}\left(\mathrm{CD}_{3}\right)_{2} \mathrm{CO}\right)$ ठ: 
113.6, 113.8, 116.2, 117.8, 120.9, 121.2, 121.5, 122.5, 123.7, 123.8, 124.0, 124.1, 124.4, 124.6, 125.3, 126.8, 132.7, 135.6, 139.3, 143.1, 145.6, 147.3, 154.2, 154.5; ${ }^{19} \mathrm{~F}(471 \mathrm{~Hz}$,

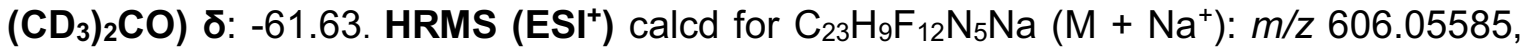
found $606.05591(0.13 \mathrm{ppm})$.

Characterisation of 2,6-bis(5,6-difluoro-1H-benzo[d]limidazol-2-yl)pyridine 4

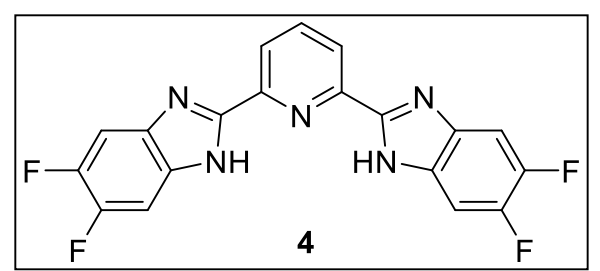

Using general procedure $\mathbf{A}$ and 4(trifluoromethyl)benzene-1,2-diamine (288 $\mathrm{mg}, 2$ mmole), anion transporter $\mathbf{4}$ was obtained as a violet solid (390 mg, 51\%). ${ }^{1} \mathrm{H}$ NMR (300 Hz, DMSO, $d_{6}$ ) ठ: 7.75-7.85 (m, 4H, ArH), $8.18(\mathrm{t}, J=7.0 \mathrm{~Hz}, 1 \mathrm{H}, \mathrm{Ar}-$ $\mathrm{H}), 8.30(\mathrm{~d}, J=7.0 \mathrm{~Hz}, 2 \mathrm{H}, \mathrm{Ar}-\mathrm{H}) ;{ }^{13} \mathrm{C}$ NMR $\left(75 \mathrm{~Hz}\right.$, DMSO, $\left.d_{6}\right): 122.0,139.9,146.2$, 147.7, 149.4, 152.8. HRMS (ESI ${ }^{+}$) calcd for $\mathrm{C}_{19} \mathrm{H}_{10} \mathrm{~F}_{4} \mathrm{~N}_{5}(\mathrm{M}+\mathrm{H}): \mathrm{m} / \mathrm{z} 384.08668$, found 384.08644 (0.65 ppm).

\section{3- General procedure B of complexes 6 and $7^{[4]}$}

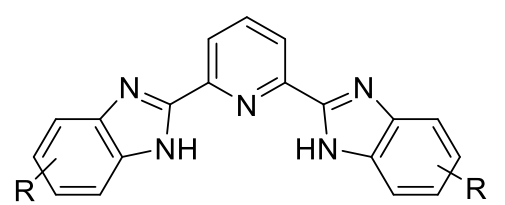

$2 \mathrm{R}=5(6)-\mathrm{CF}_{3}$ $3 \mathrm{R}=4(5), 6(7)-\mathrm{BisCF}_{3}$

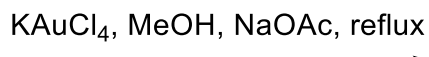

Scheme S3: Synthesis of gold chloride complexes 6 and 7

Dry methanol $(25 \mathrm{~mL})$ was added a mixture of anion receptor $2(224 \mathrm{mg}, 0.50 \mathrm{mmol})$ or anion receptor $3(292 \mathrm{mg}, 0.50 \mathrm{mmol})$, dried sodium acetate $(325 \mathrm{mg}, 3.97 \mathrm{mmol})$ and $\mathrm{KAuCl}_{4}(284 \mathrm{mg}, 0.75 \mathrm{mmol})$ and was heated at reflux for $3 \mathrm{~h}$. The resulting solid was filtered and washed with methanol $(2 \times 10 \mathrm{~mL})$ and diethyl ether $(3 \times 10 \mathrm{~mL})$ and dried to give complex 6 (352 mg, 67\%) and 7 ( $455 \mathrm{mg}, 56 \%$ ) as yellow powders. 


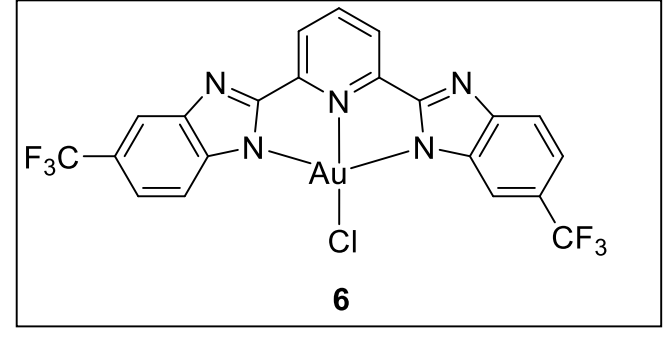

${ }^{1} \mathrm{H}$ NMR (500 Hz, DMSO, $\left.d_{6}\right)$ ס: 7.56 (td, $J=2$, $7 \mathrm{~Hz}, 1 \mathrm{H}, \mathrm{Ar}-\mathrm{H}$ ), 7.70 (dd, $J=2.0,9.0 \mathrm{~Hz}, 1 \mathrm{H}$, Ar-H), 7.95-7.98 (m, 1H, Ar-H), 8.14 (s, 1H, Ar$\mathrm{H}), 8.23(\mathrm{t}, J=8.0 \mathrm{~Hz}, 1 \mathrm{H}, \mathrm{Ar}-\mathrm{H}), 8.33-8.40(\mathrm{~m}$, $3 \mathrm{H}, \mathrm{Ar}-\mathrm{H}), 8.61-8.66(\mathrm{~m}, 1 \mathrm{H}, \mathrm{Ar}-\mathrm{H}) ;{ }^{13} \mathrm{C}$ NMR

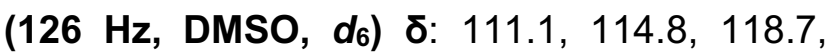

119.5, 122.2, 123.4, 123.5, 124.0, 142.2, 126.1, 140.0, 142.4, 142.8, 145.3, 146.7, 146.8, 147.6, 160.4, 160.8; ${ }^{19} \mathrm{~F}$ (471 Hz, DMSO) $\delta:-59.62,59.26$. HRMS (APPI) calcd for $\mathrm{C}_{21} \mathrm{H}_{9} \mathrm{AuClF}_{6} \mathrm{~N}_{5}\left(\mathrm{M}^{+}\right): \mathrm{m} / \mathrm{z} 677.01109$, found 677.01068 (0.60 ppm).

Characterisation of complex 7

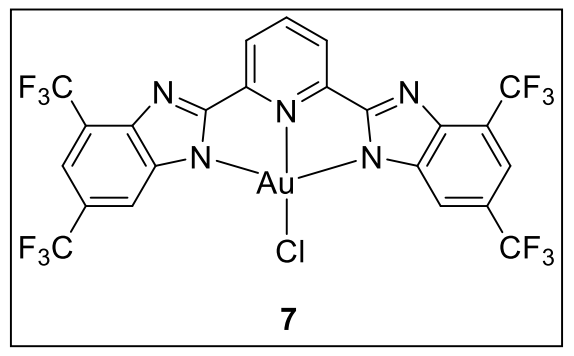

${ }^{1} \mathrm{H}$ NMR $\left(500 \mathrm{~Hz},\left(\mathrm{CD}_{3}\right)_{2} \mathrm{CO}\right)$ ס: $7.75(\mathrm{~s}, 2 \mathrm{H}, \mathrm{Ar}-\mathrm{H})$, $8.41(\mathrm{~d}, J=5.0 \mathrm{~Hz}, 2 \mathrm{H}, \mathrm{Ar}-\mathrm{H}), 8.58(\mathrm{~s}, 2 \mathrm{H}, \mathrm{Ar}-\mathrm{H}), 8.73$ $(\mathrm{t}, J=8.0 \mathrm{~Hz}, 1 \mathrm{H}, \mathrm{Ar}-\mathrm{H}) ;{ }^{13} \mathrm{C}$ NMR $\left(126 \mathrm{~Hz},\left(\mathrm{CD}_{3}\right)_{2} \mathrm{CO}\right)$ ठ: $115.3\left(\mathrm{~d}, \mathrm{~J}^{1} \mathrm{C}-\mathrm{F}=200 \mathrm{~Hz}\right), 120.8,122.1\left(\mathrm{~d}, \boldsymbol{J}^{2} \mathrm{C}-\mathrm{F}=16\right.$ $\mathrm{Hz}), 122.9,124.4\left(\mathrm{~d}, \mathrm{~J}^{3} \mathrm{C}-\mathrm{F}=8 \mathrm{~Hz}\right), 125.1,125.7$ (d, $\left.J^{1} \mathrm{C}-\mathrm{F}=200 \mathrm{~Hz}\right), 125.9\left(\mathrm{~d}, \mathrm{~J}^{2} \mathrm{C}-\mathrm{F}=33 \mathrm{~Hz}\right), 127.3,141.1$, 141.7, 147.0, 147.8, 161.8. ${ }^{19} \mathrm{~F}\left(\mathbf{4 7 1} \mathrm{Hz},\left(\mathrm{CD}_{3}\right)_{2} \mathrm{CO}\right) \mathbf{\delta}$ : -61.92. HRMS (APCI) calcd for $\mathrm{C}_{23} \mathrm{H}_{7} \mathrm{AuClF}_{12} \mathrm{~N}_{5}\left(\mathrm{M}^{+}\right): \mathrm{m} / \mathrm{z}$ 812.98586, found 812.98982 (4.9 ppm).

\section{4- General procedure $C$ of complexes 8 and $9^{[4]}$}

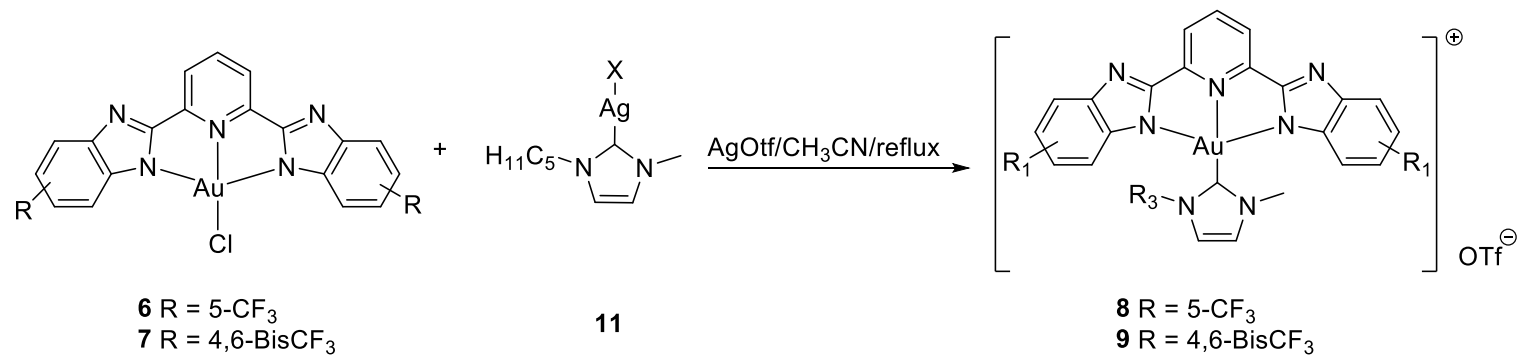

Scheme S4: Synthesis of gold III NHC complexes 8 and 9 
Previously dried silver triflate $(46 \mathrm{mg}, 0.18 \mathrm{mmol}$ ) was added to a suspension of (1-methyl3-pentyl-1,3-dihydro-2 $\mathrm{H}$-imidazol-2-ylidene)silver(I)bromide (60 mg, $0.18 \mathrm{mmole}$ ) and complex 6 (100 mg, $0.15 \mathrm{mmol}$ ) or complex 7 (120 mg, $0.15 \mathrm{mmol})$ in acetonitrile (15 mL). After holding the reaction mixture in the dark at reflux for $12 \mathrm{~h}$, it was cooled, filtered and purified by cycles of crystallization of diethyl ether diffusion in an acetonitrile solution of the product.

\section{Characterisation of complex 8}

Using general procedure B, complex 8 was obtained as a yellow solid (18 mg, 13\%).

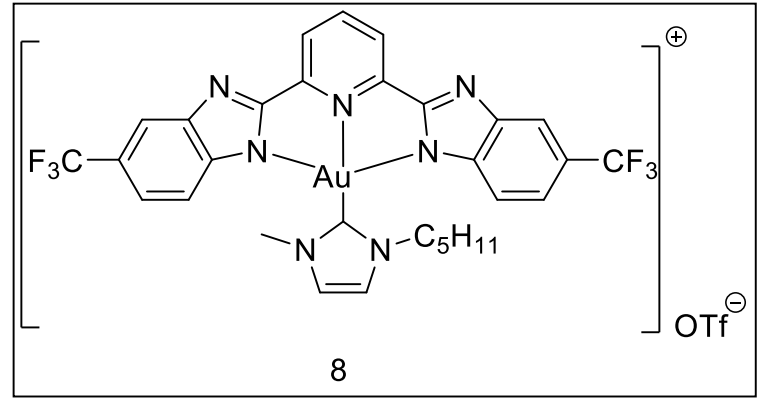

${ }^{1} \mathrm{H}$ NMR (300 MHz, $\left.\mathrm{CD}_{3} \mathrm{CN}\right) \delta: 0.51(\mathrm{t}, \mathrm{J}=$ $\left.7.0 \mathrm{~Hz}, 3 \mathrm{H}, \mathrm{CH}_{3}\right), 0.98-1.05\left(\mathrm{~m}, 2 \mathrm{H}, \mathrm{CH}_{2}\right)$, 1.13-1.18 (m, 2H, $\left.\mathrm{CH}_{2}\right), 1.71-1.80(\mathrm{~m}, 2 \mathrm{H}$, $\left.\mathrm{CH}_{2}\right), 4.05\left(\mathrm{~s}, 3 \mathrm{H}, \mathrm{CH}_{3}\right), 4.36-4.41(\mathrm{~m}, 2 \mathrm{H}$, $\left.\mathrm{CH}_{2}\right), 6.12-6.20(\mathrm{~m}, 2 \mathrm{H}, \mathrm{Ar}-\mathrm{H}), 7.53-7.62$ $(m, 2 \mathrm{H}, \mathrm{Ar}-\mathrm{H}), 7.93-8.03(\mathrm{~m}, 2 \mathrm{H}, \mathrm{Ar}-\mathrm{H})$,

$8.22(\mathrm{~s}, 1 \mathrm{H}, \mathrm{Ar}-\mathrm{H}), 8.39$ (d, J = 8.0 Hz, 2H, Ar-H), $8.64(\mathrm{t}, J=8.0 \mathrm{~Hz}, 1 \mathrm{H}, \mathrm{Ar}-\mathrm{H}) ;{ }^{13} \mathrm{C}$ NMR

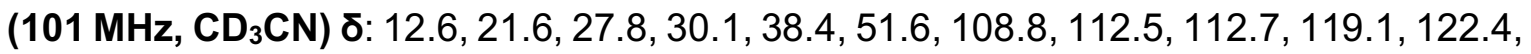
122.9, 125.5, 126.9, 138.3, 141.2, 142.3, 147.2, 147.2, 147.5, 161.6; HRMS (APPI) calcd for $\mathrm{C}_{30} \mathrm{H}_{25} \mathrm{AuF}_{6} \mathrm{~N}_{7}{ }^{+}$(M - OTf) ${ }^{+}: \mathrm{m} / \mathrm{z}$ 794.17358, found 794.17369 (0.10 ppm).

\section{Characterisation of complex 9}

Using general procedure B, complex 9 was obtained as a yellow solid (20 mg, $12 \%$ ).

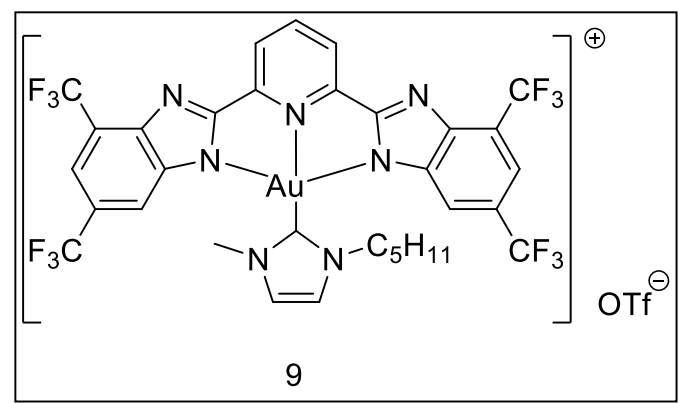

${ }^{1} \mathrm{H}$ NMR $\left(300 \mathrm{~Hz},\left(\mathrm{CD}_{3}\right)_{2} \mathrm{CO}\right) \mathrm{\delta}$ :

$0.39\left(\mathrm{t}, J=7.0 \mathrm{~Hz}, 3 \mathrm{H}, \mathrm{CH}_{3}\right), 0.85-1.03(\mathrm{~m}, 2 \mathrm{H}$, $\left.\mathrm{CH}_{2}\right), 1.04-1.11\left(\mathrm{~m}, 2 \mathrm{H}, \mathrm{CH}_{2}\right), 1.72-1.79(\mathrm{~m}, 2 \mathrm{H}$, $\left.\mathrm{CH}_{2}\right), 4.26\left(\mathrm{~s}, 3 \mathrm{H}, \mathrm{CH}_{3}\right), 4.63(\mathrm{t}, J=7.0 \mathrm{~Hz}, 2 \mathrm{H}$, $\left.\mathrm{CH}_{3}\right), 6.45(\mathrm{~s}, 2 \mathrm{H}, \mathrm{Ar}-\mathrm{H}), 7.77(\mathrm{~s}, 1 \mathrm{H}, \mathrm{Ar}-\mathrm{H}), 7.88$

(s, $1 \mathrm{H}, \mathrm{Ar}-\mathrm{H}$ ), 8.37 (d, J = 2.0 Hz, 2H, Ar-H), 8.42 (d, $J=2.0 \mathrm{~Hz}, 2 \mathrm{H}, \operatorname{Ar}-\mathrm{H}), 8.51$ (d, $J=$ $8.0 \mathrm{~Hz}, 2 \mathrm{H}, \mathrm{Ar}-\mathrm{H}), 8.75(\mathrm{t}, \mathrm{J}=8.0 \mathrm{~Hz}, 1 \mathrm{H}, \mathrm{Ar}-\mathrm{H}) ;{ }^{13} \mathrm{C} \mathrm{NMR}\left(75 \mathrm{MHz},\left(\mathrm{CD}_{3}\right)_{2} \mathrm{CO}\right)$ ठ: 12.6 , $21.7,27.9,30.4,38.5,51.8,113.0,116.8,119.8,119.9,120.5,122.1,122.8$ (q, ${ }^{2} J_{C, F}=34$ $\mathrm{Hz}), 124.2,124.5$ (q, $\left.{ }^{1} \mathrm{~J}_{\mathrm{C}, \mathrm{F}}=209 \mathrm{~Hz}\right), 127.6,131.9,139.8,141.5,147.4,147.6,163.9$; 
HRMS (APPI) calcd for $\mathrm{C}_{32} \mathrm{H}_{23} \mathrm{AuF}_{12} \mathrm{~N}_{7}{ }^{+}(\mathrm{M}-\mathrm{OTf})^{+}: \mathrm{m} / \mathrm{z}$ 930.14835, found 930.14861 (0.50 ppm). 


\section{S3: ${ }^{1} \mathrm{H}$ NMR and ${ }^{13} \mathrm{C}$ NMR Data}

Figure S1: ${ }^{1} \mathrm{H}$ NMR of 2,6-bis(1H-benzo[d]imidazol-2-yl)pyridine

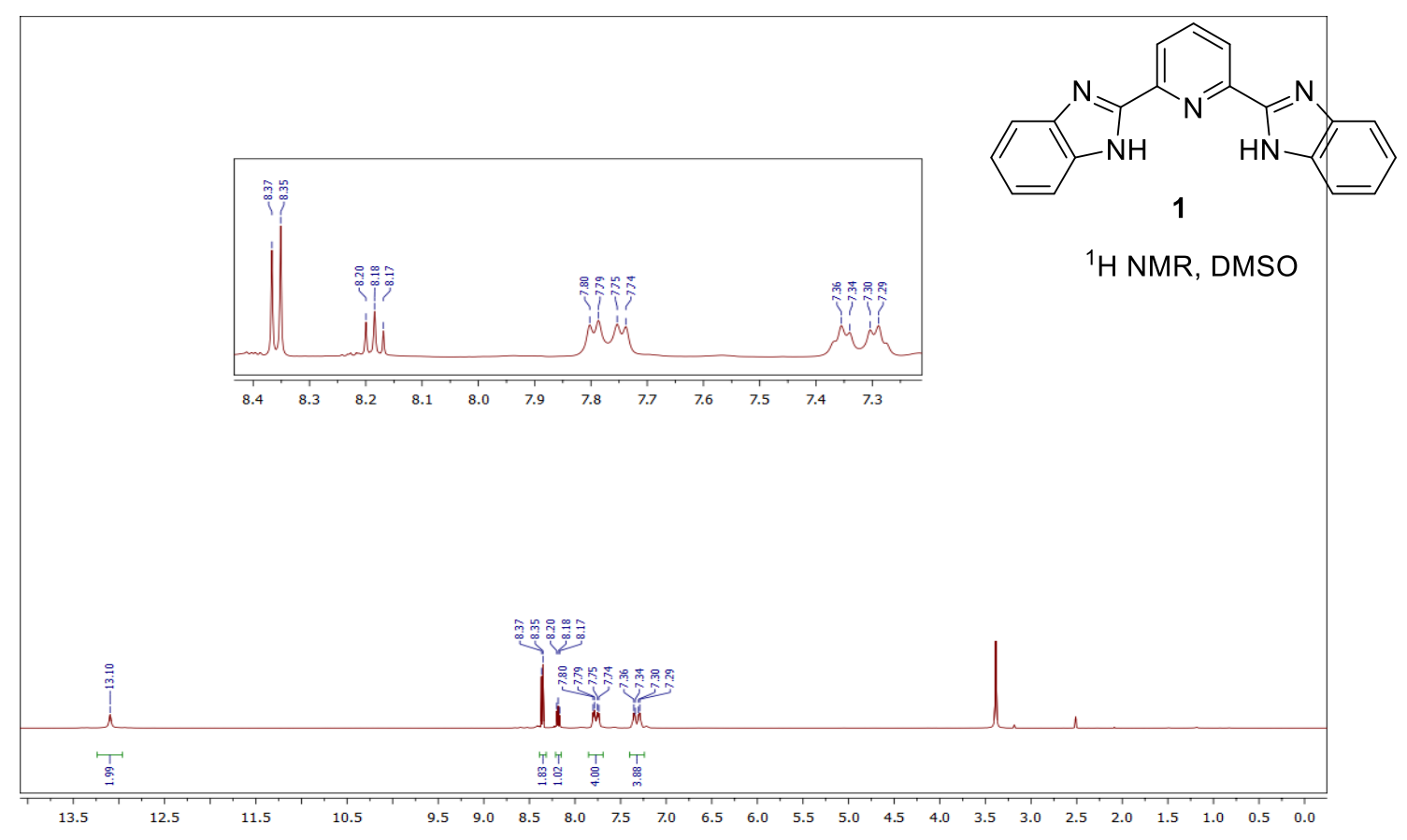

Figure S2: ${ }^{13} \mathrm{C}$ NMR of 2,6-bis(1H-benzo[d]imidazol-2-yl)pyridine

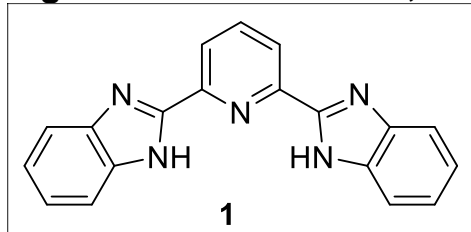

${ }^{13} \mathrm{C}$ NMR, DMSO

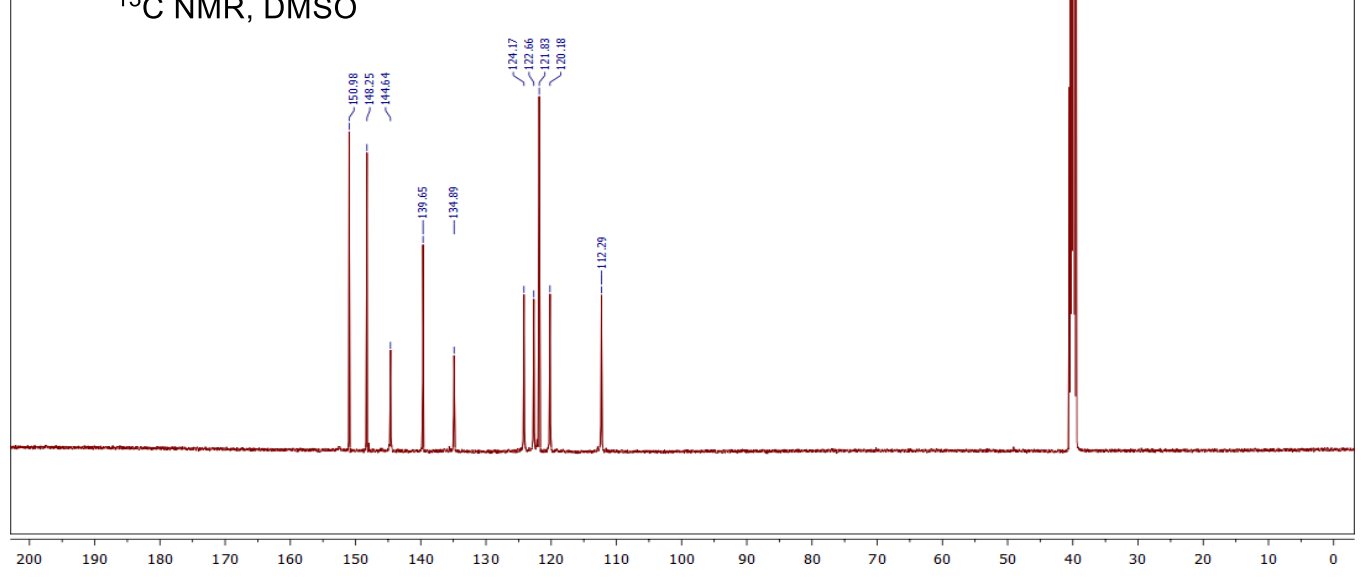


Figure S3: COSY of 2,6-bis(1H-benzo[d]imidazol-2-yl)pyridine

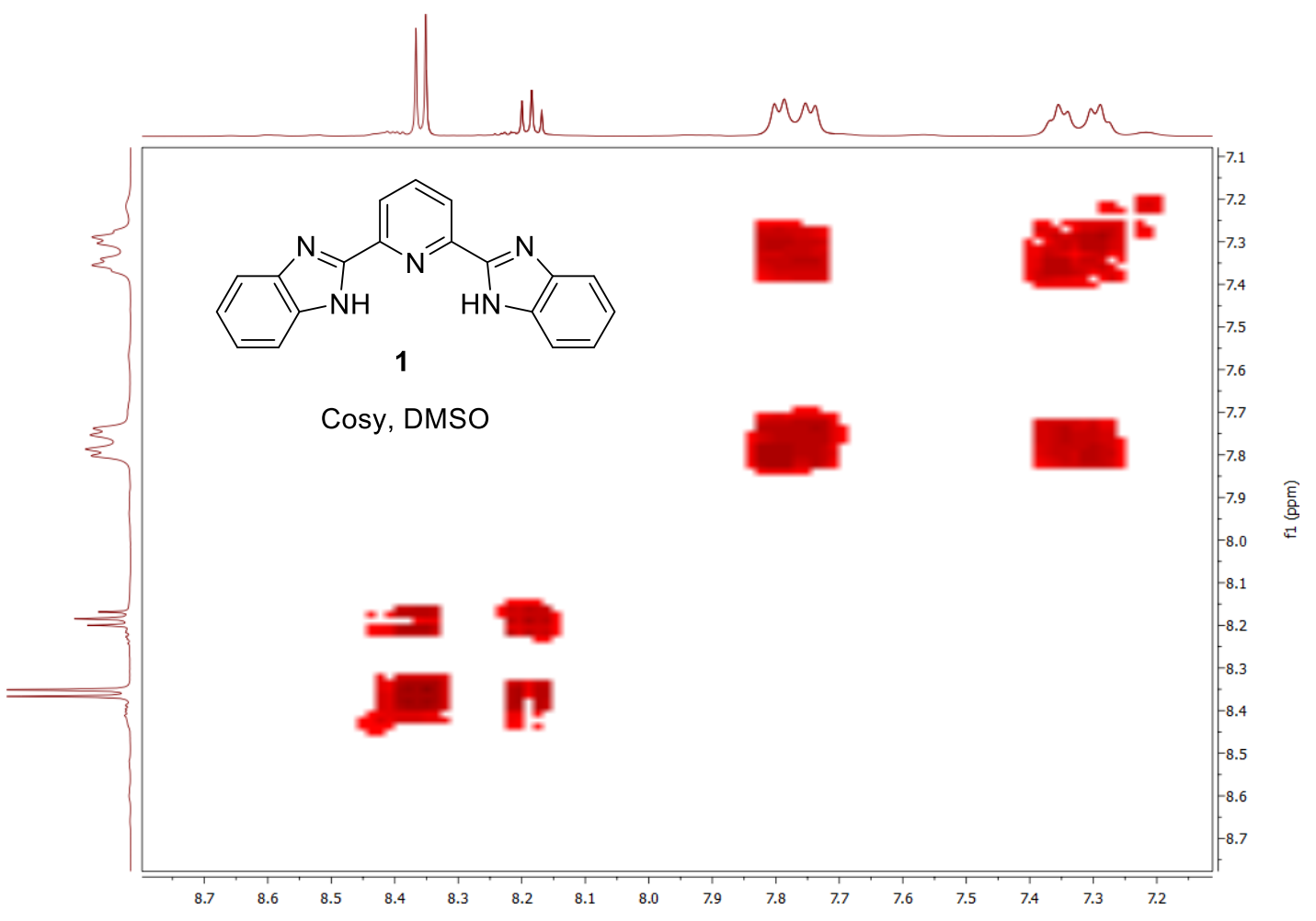

Figure S4: HSQC of 2,6-bis(1H-benzo[d]imidazol-2-yl)pyridine

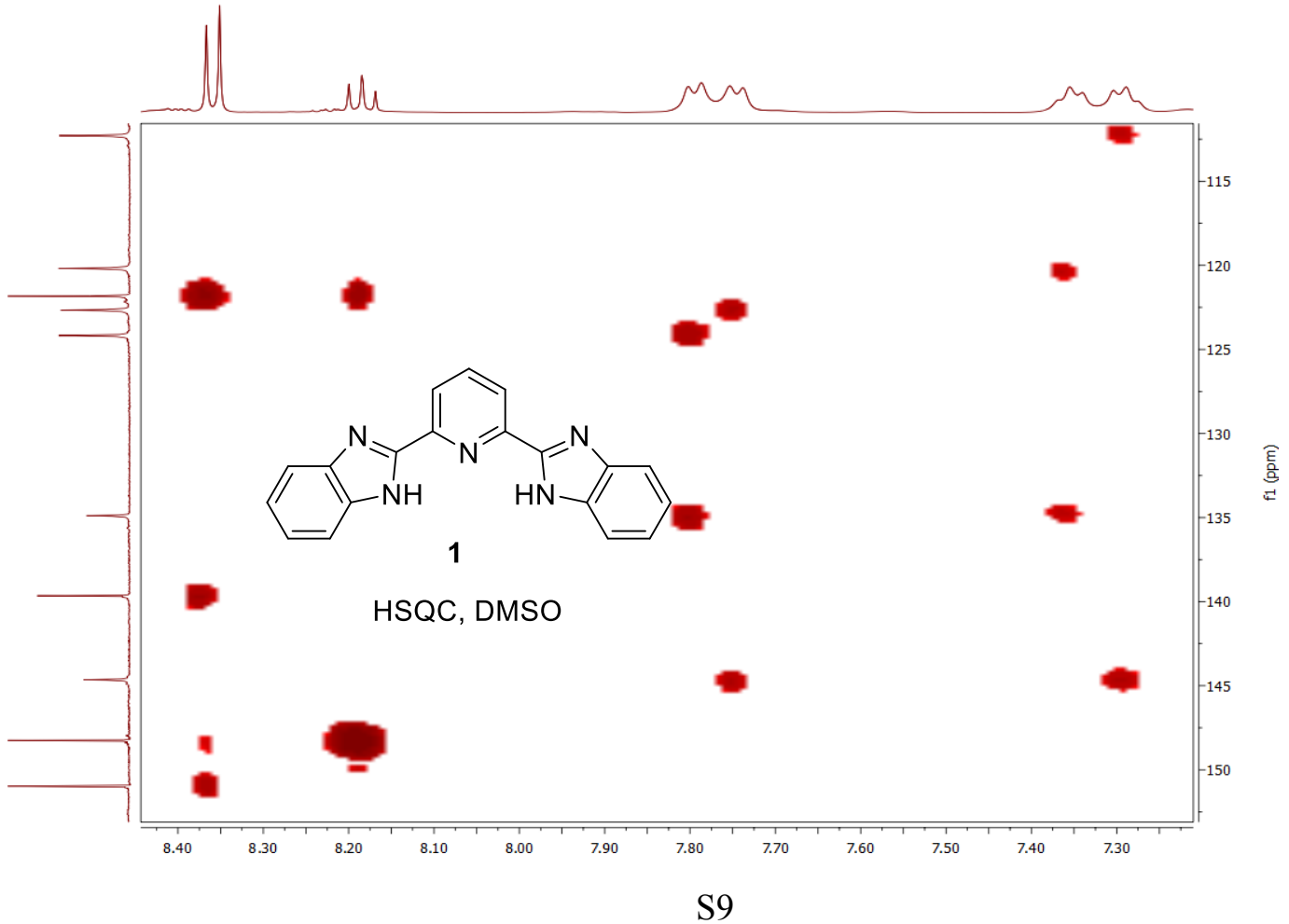


Figure S5: ${ }^{1} \mathrm{H}$ NMR of 2,6-bis(5-(trifluoromethyl)-1H-benzo[d]imidazol-2-yl)pyridine 2

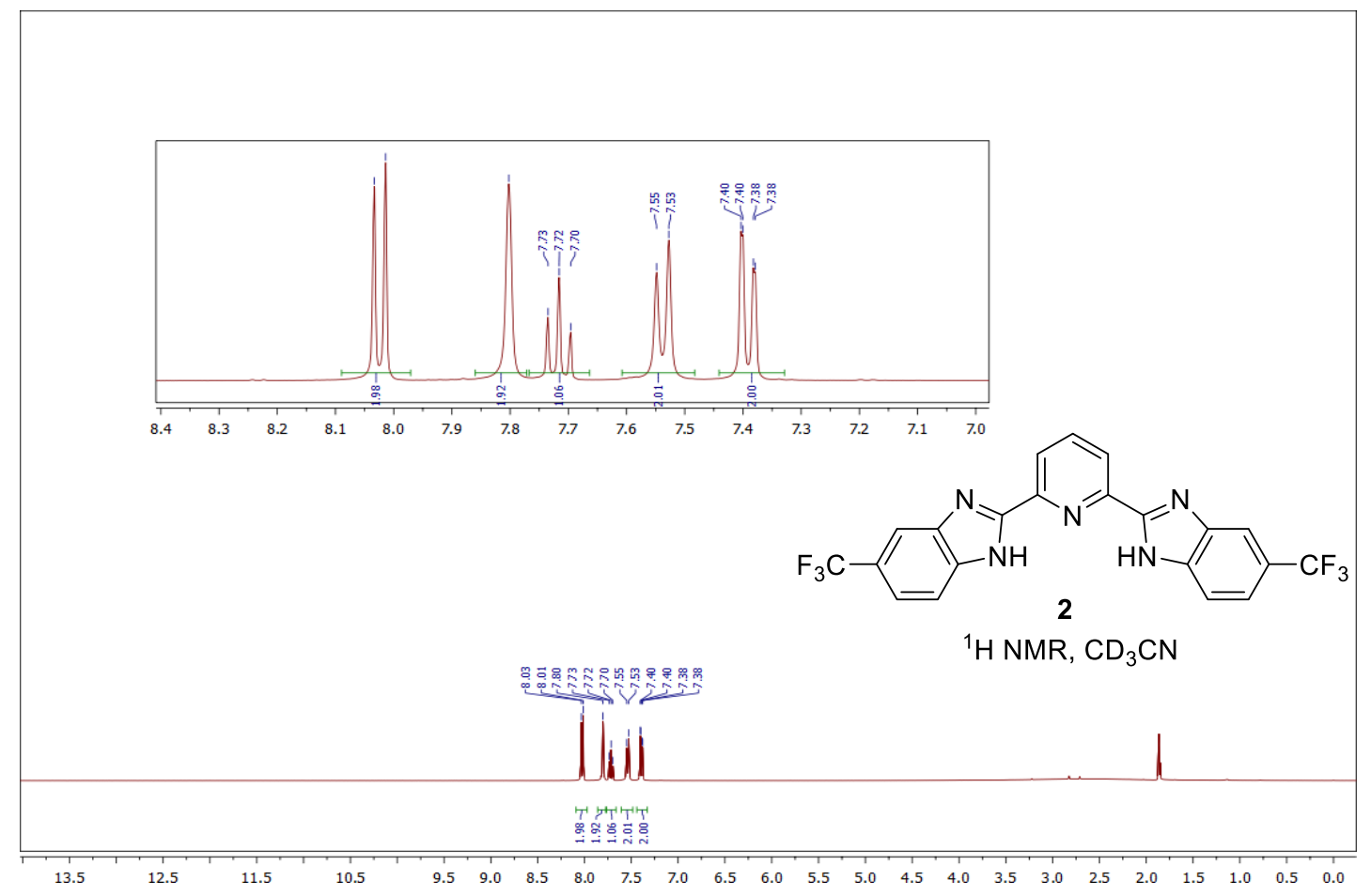

Figure S6: ${ }^{13} \mathrm{C}$ NMR of 2,6-bis(5-(trifluoromethyl)-1H-benzo[d]imidazol-2-yl)pyridine 2

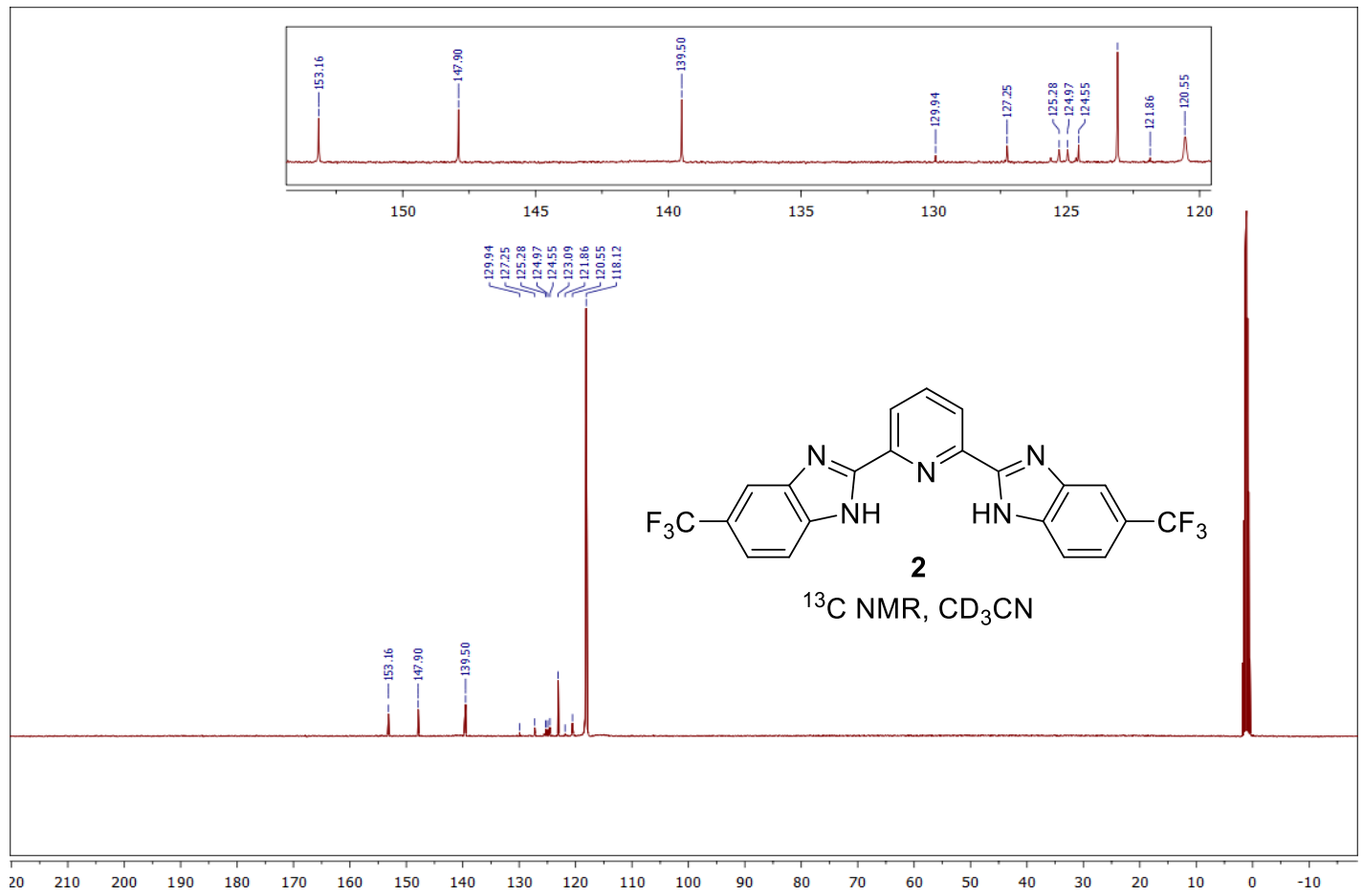


Figure S7: ${ }^{1} \mathrm{H}$ NMR of 2,6-bis(5-(trifluoromethyl)-1H-benzo[d]imidazol-2-yl)pyridine 2

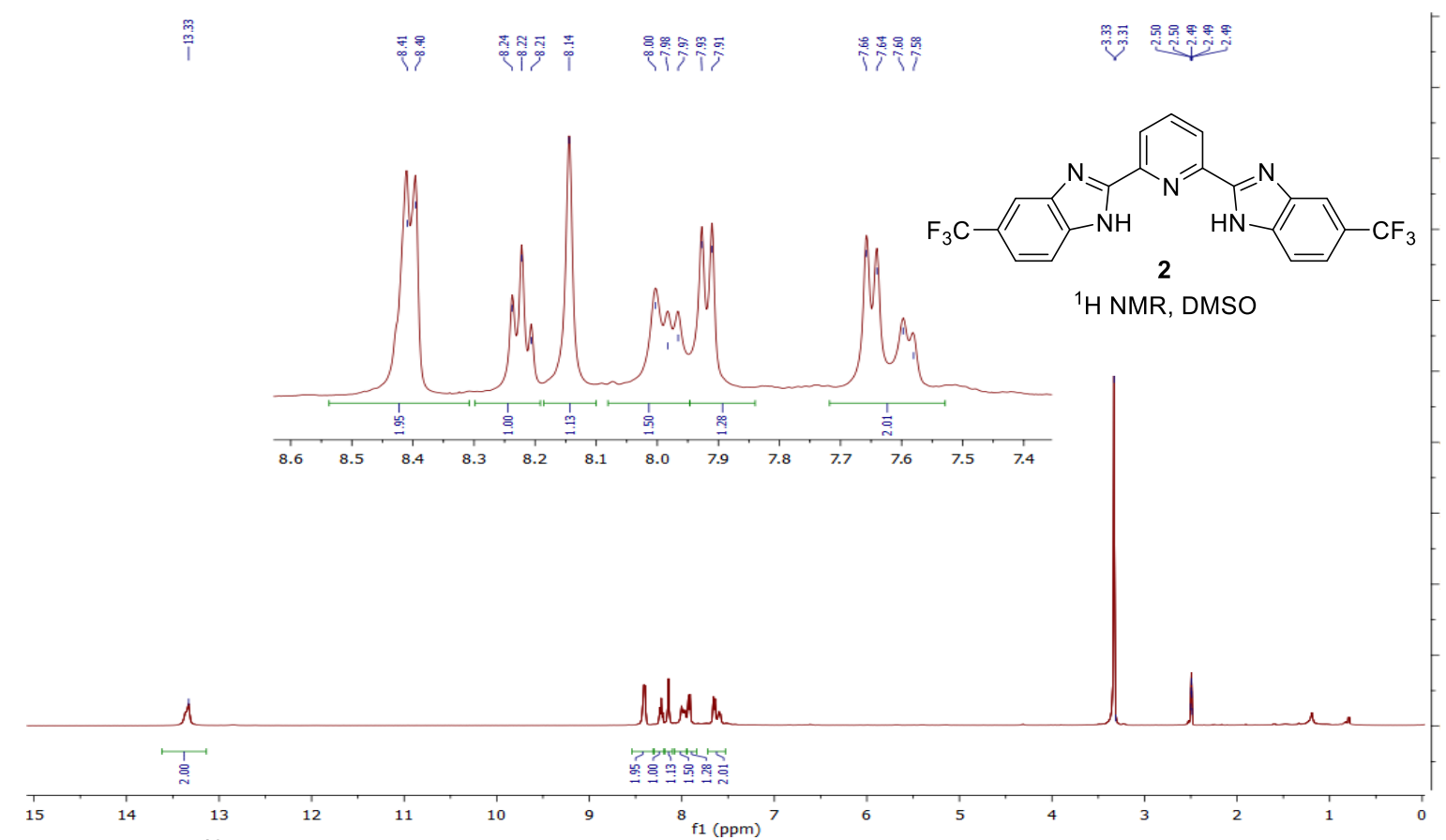

Figure S8: ${ }^{13} \mathrm{C}$ NMR of 2,6-bis(5-(trifluoromethyl)-1H-benzo[d]imidazol-2-yl)pyridine 2

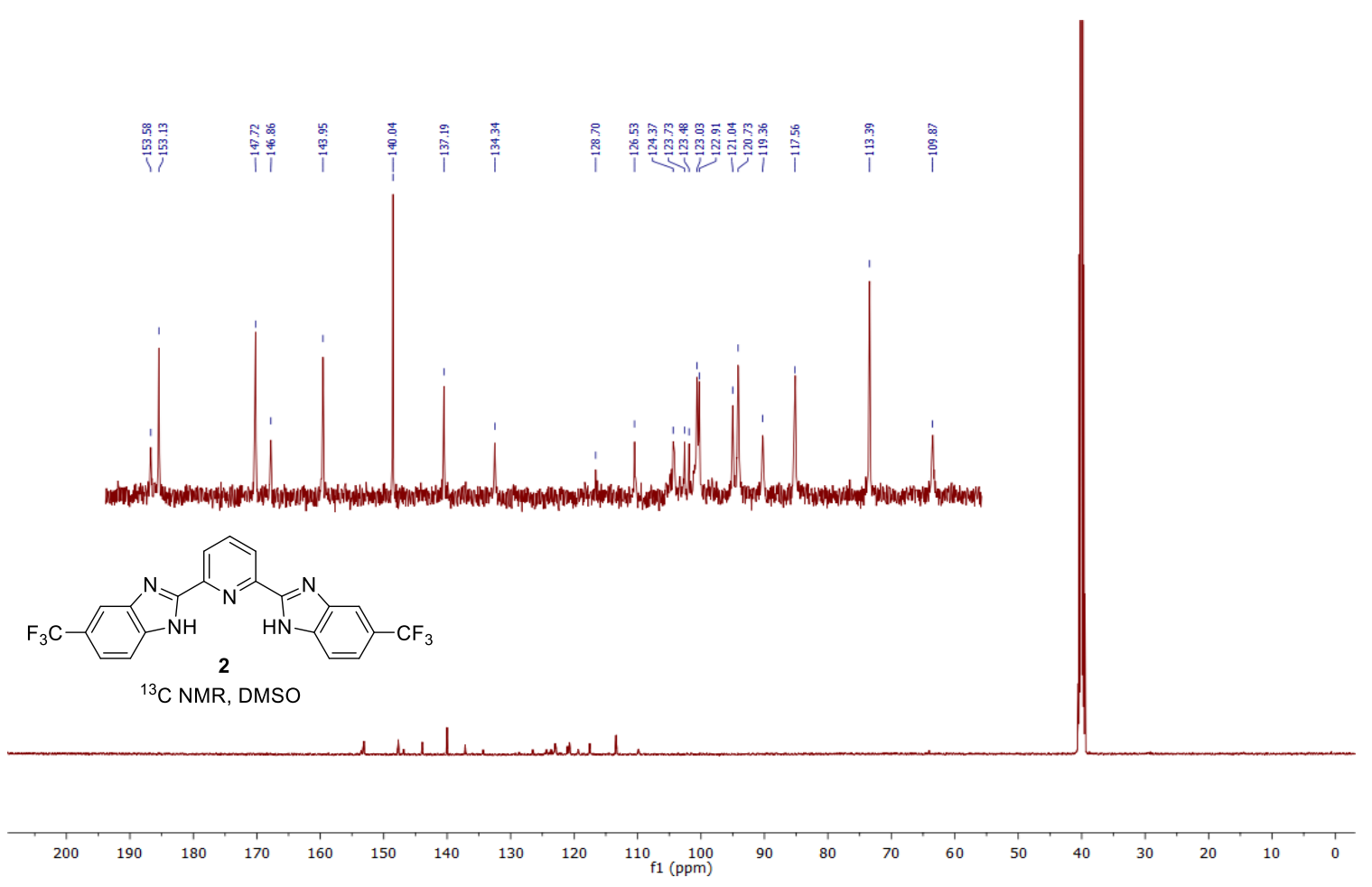


Figure S9: ${ }^{1} \mathrm{H}$ NMR of 2,6-bis(4,6-bis(trifluoromethyl)-1H-benzo[d]imidazol-2-yl)pyridine 3

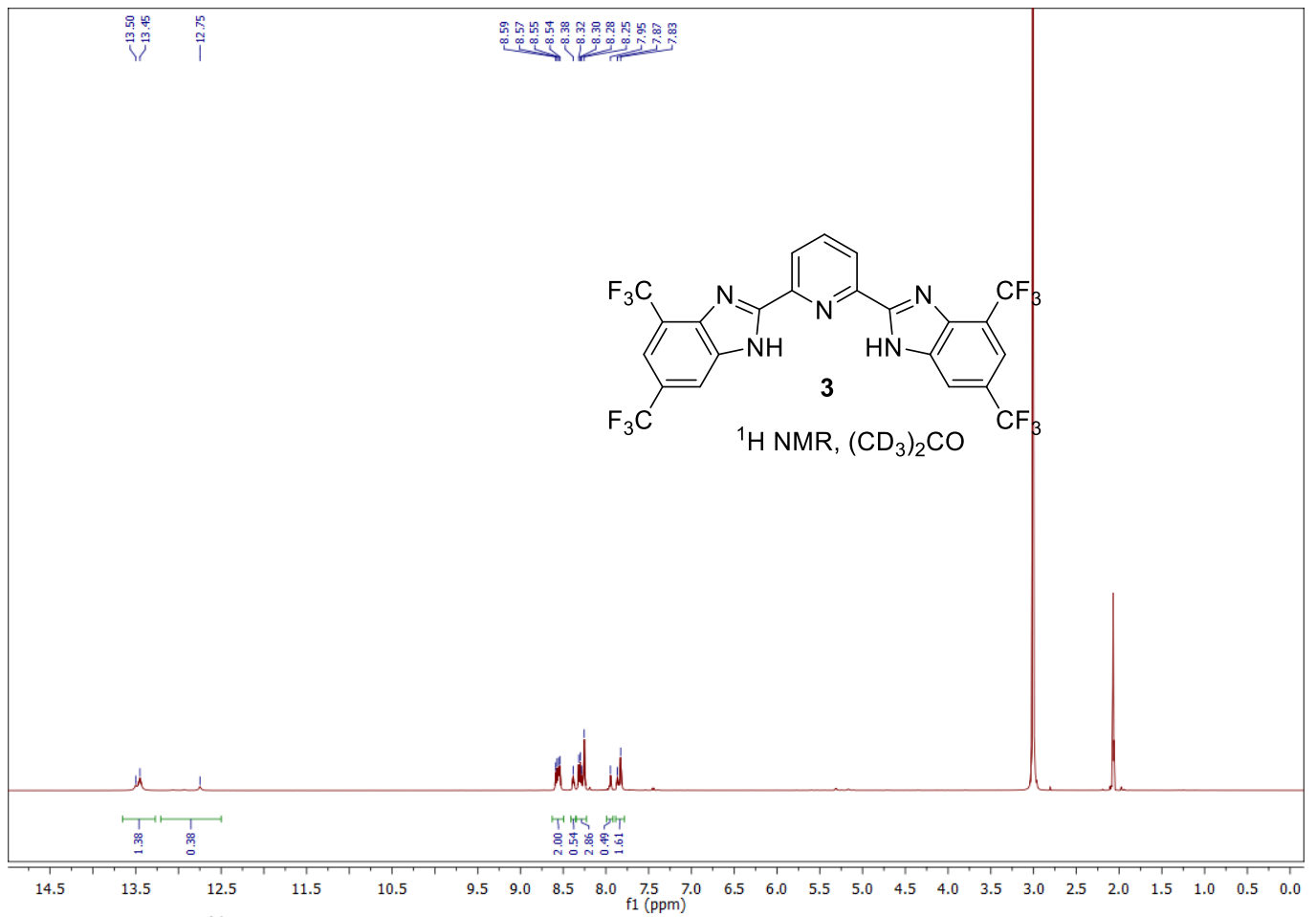

Figure S10: ${ }^{13} \mathrm{C}$ NMR of 2,6-bis(4,6-bis(trifluoromethyl)-1H-benzo[d]imidazol-2-yl)pyridine

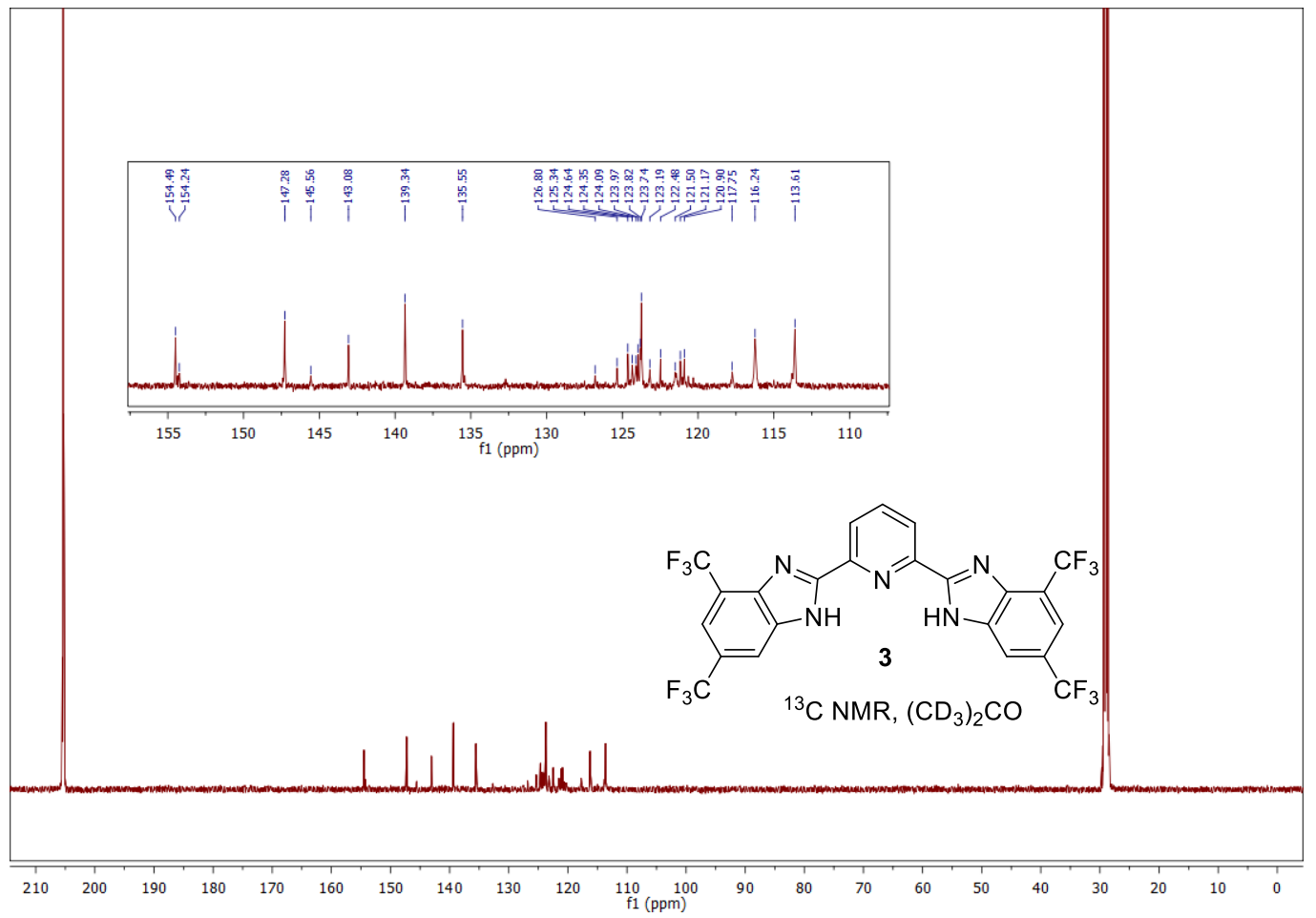


Figure S11: ${ }^{13} \mathrm{~F}$ NMR of 2,6-bis(4,6-bis(trifluoromethyl)-1H-benzo[d]imidazol-2-yl)pyridine

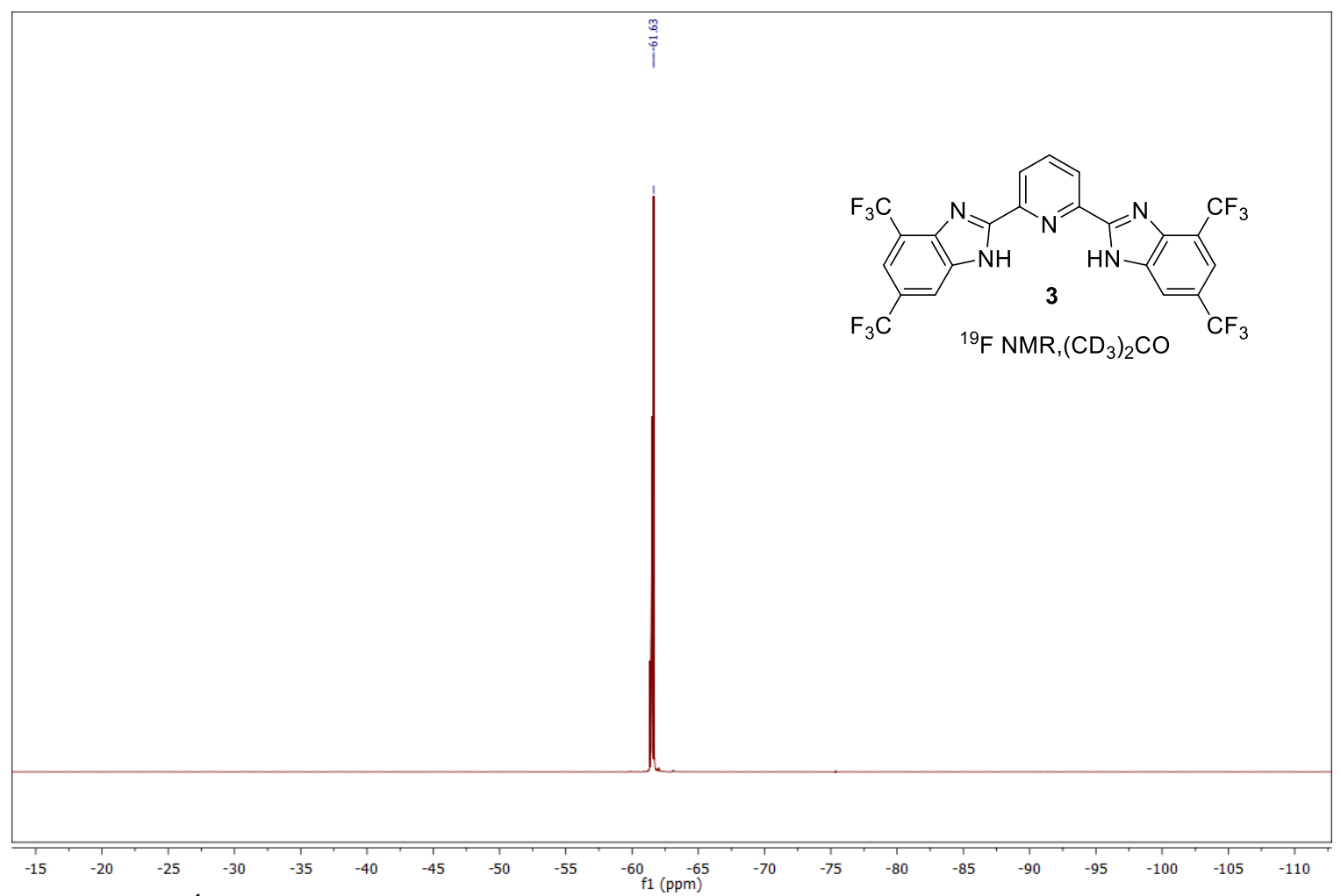

Figure S12: ${ }^{1} \mathrm{H}$ NMR of 2,6-bis(4,6-bis(trifluoromethyl)-1 H-benzo[d] imidazol-2-yl)pyridine 3

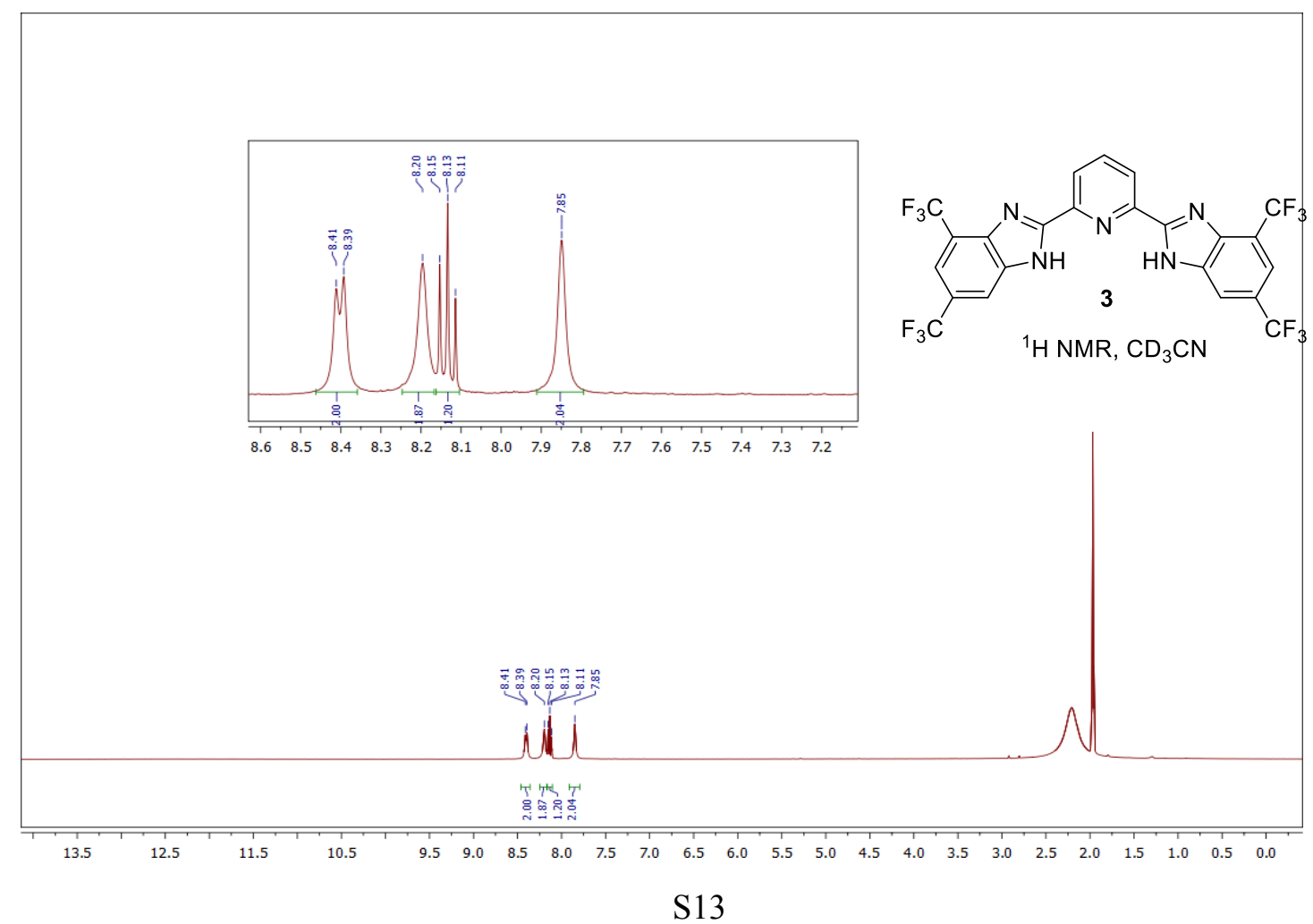


Figure S13: ${ }^{1} \mathrm{H}$ NMR of 2,6-bis(5,6-difluoro-1H-benzo[d]imidazol-2-yl)pyridine 4

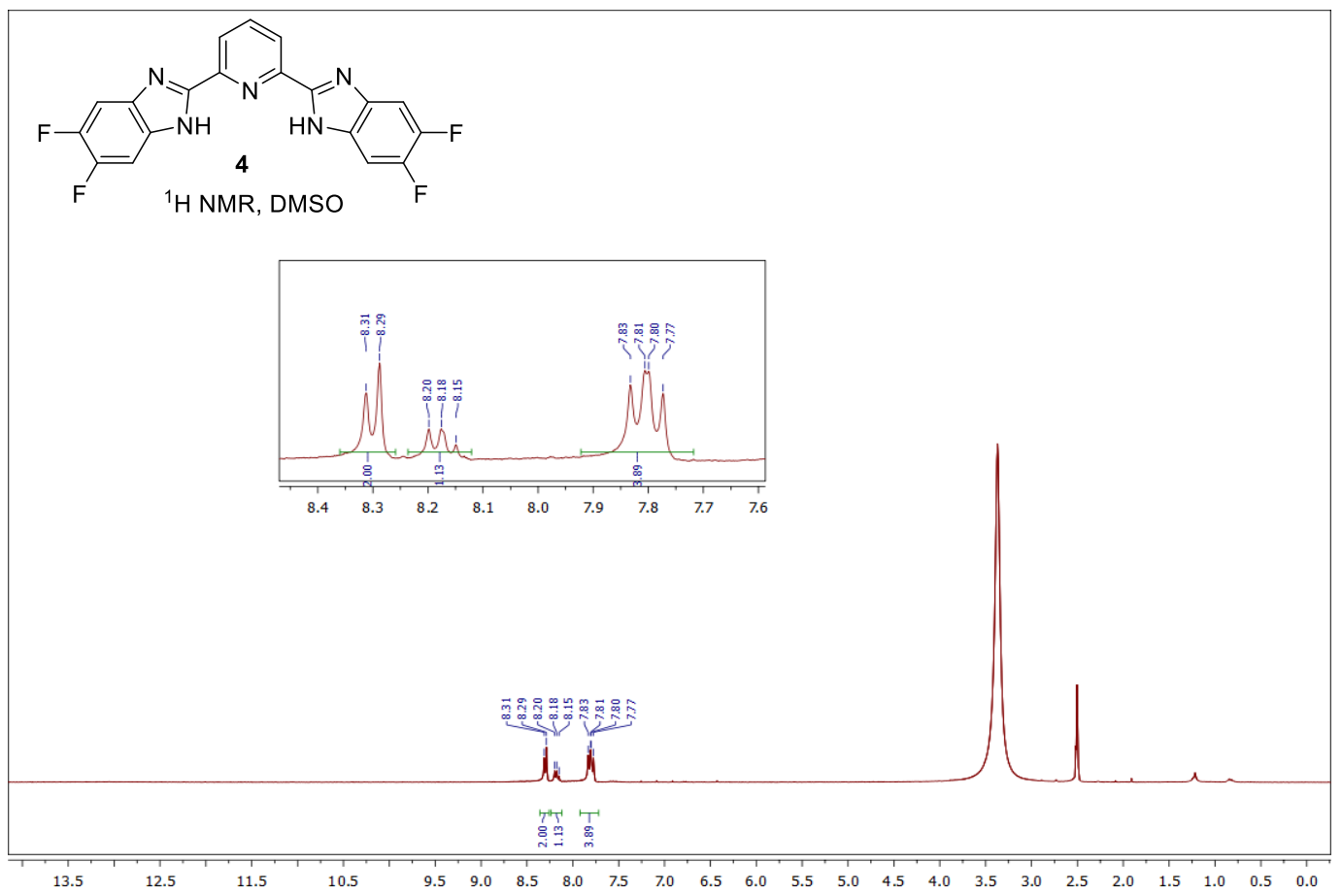

Figure S14: ${ }^{13} \mathrm{C}$ NMR of 2,6-bis(5,6-difluoro-1 $H$-benzo[d]imidazol-2-yl)pyridine 4

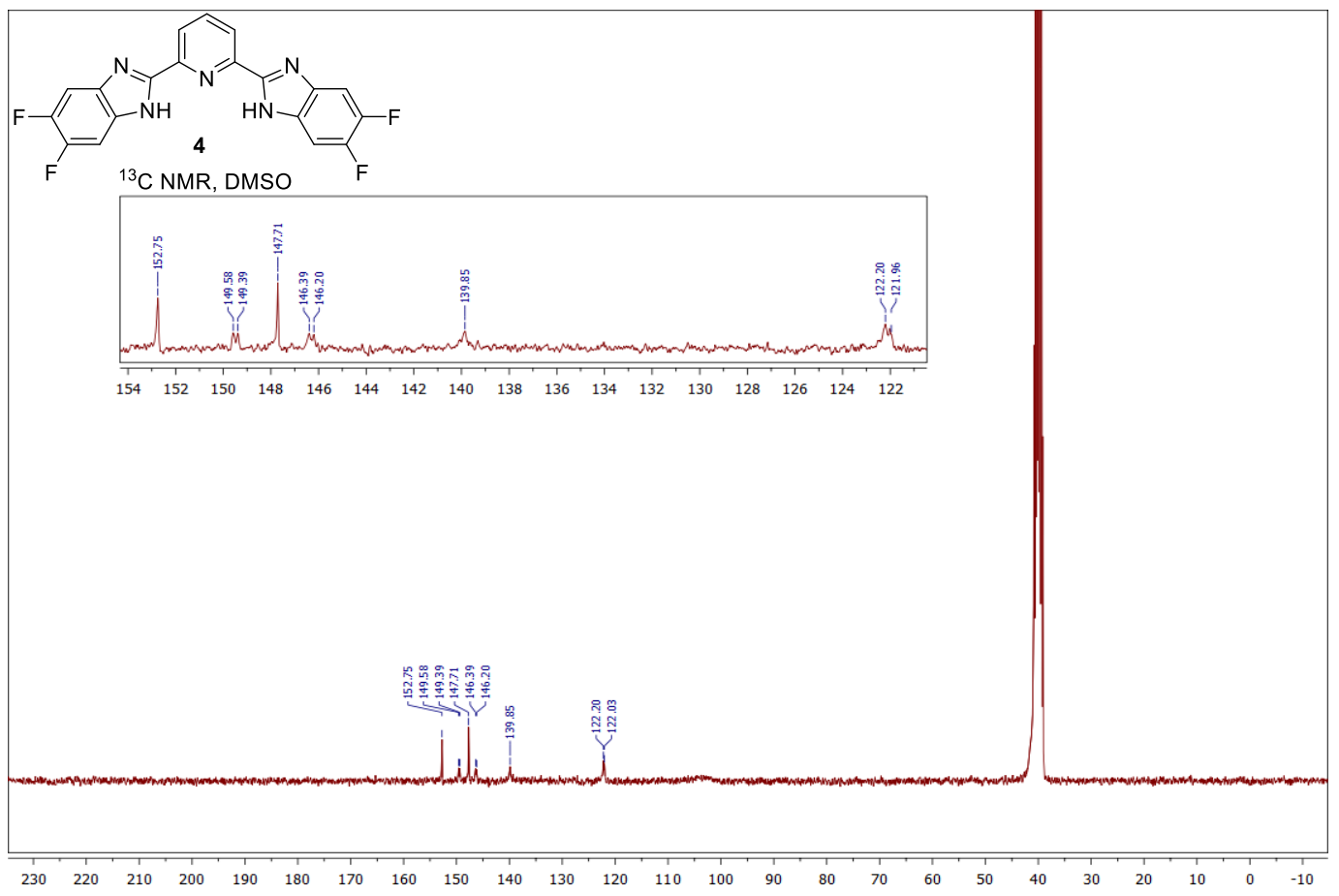


Figure S15: ${ }^{1} \mathrm{H}$ NMR of Complex 6

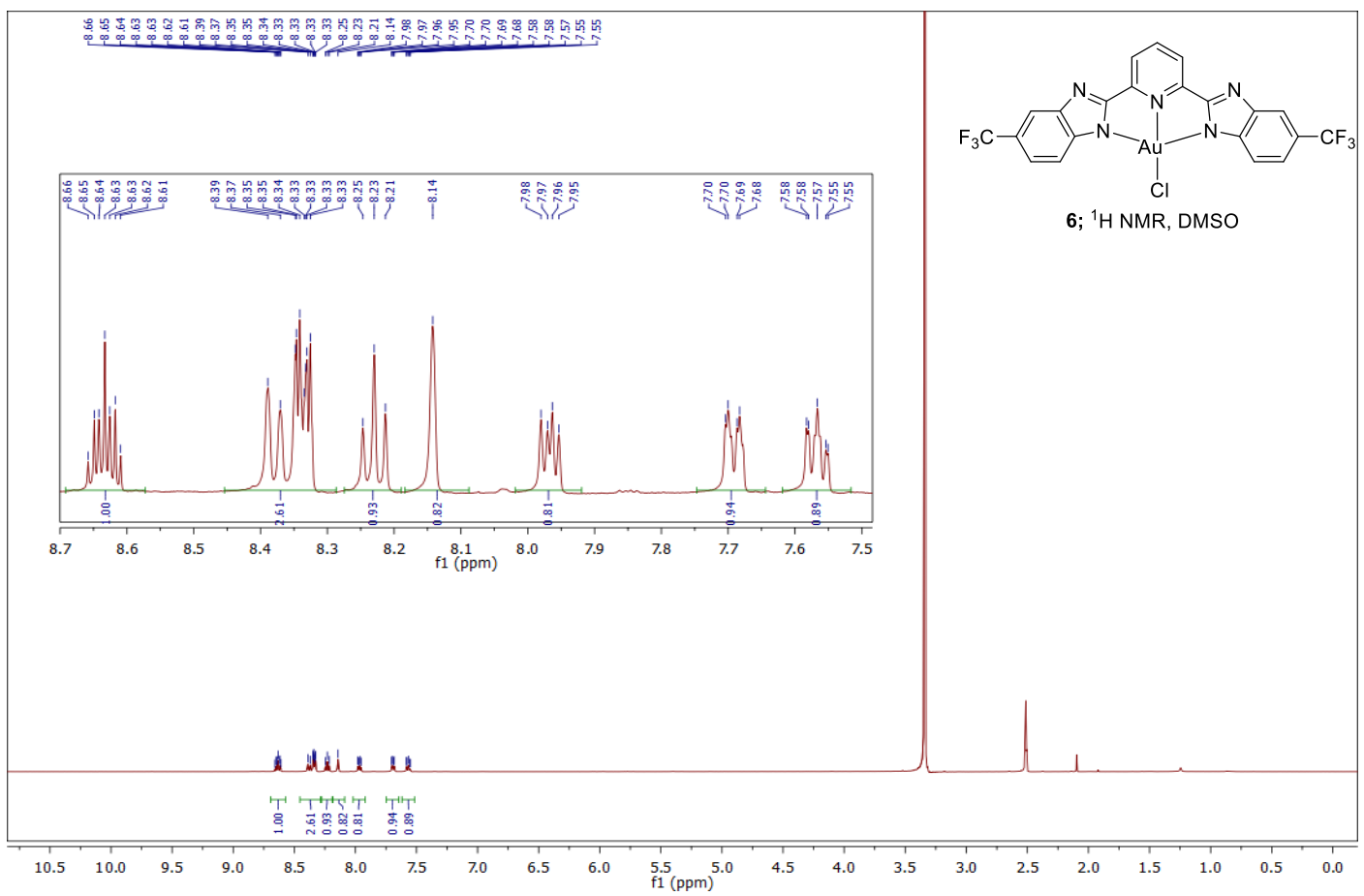

Figure S16: ${ }^{13} \mathrm{C}$ NMR of Complex 6

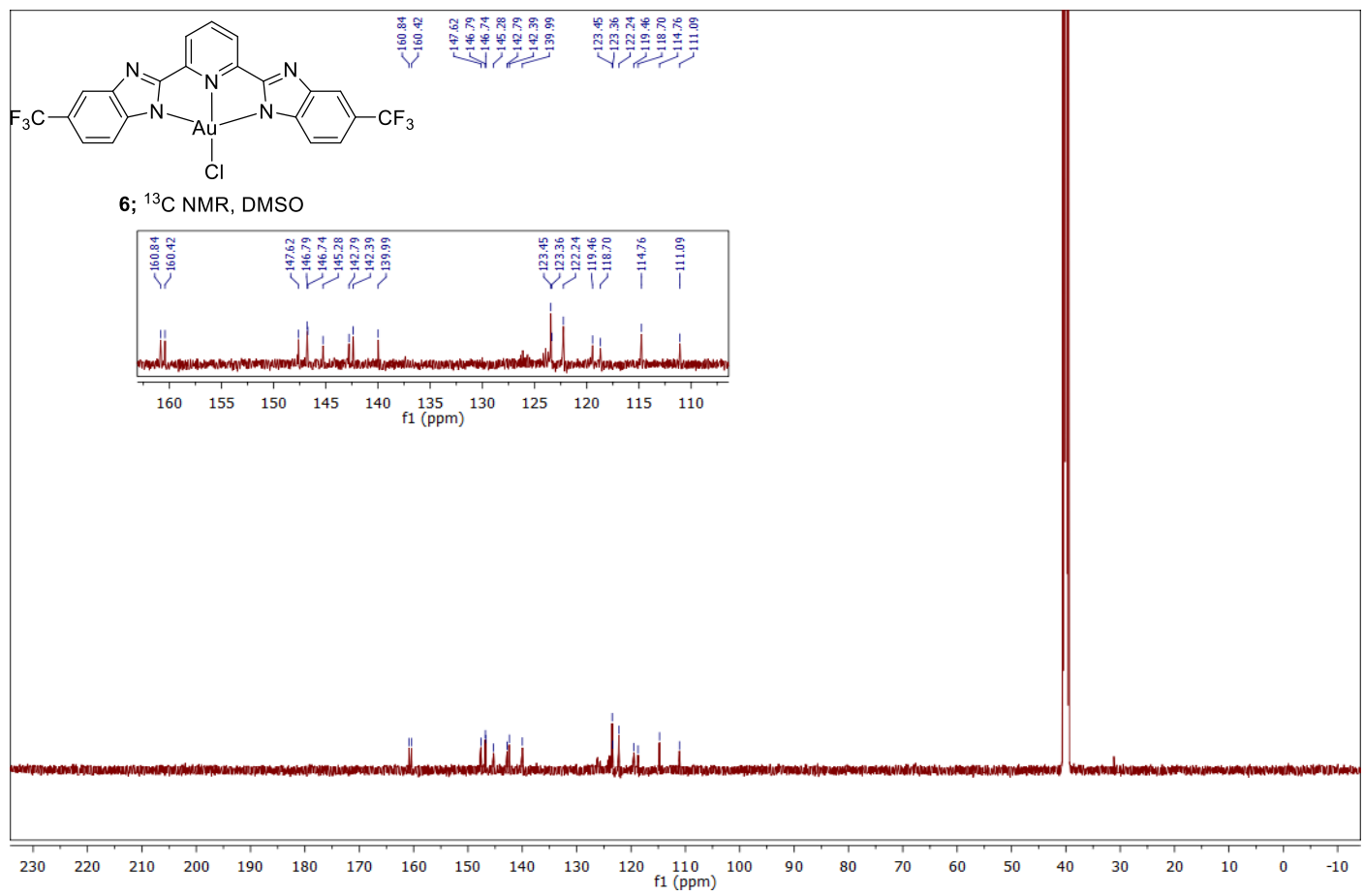


Figure S17: ${ }^{1} \mathrm{H}$ NMR of Complex 7

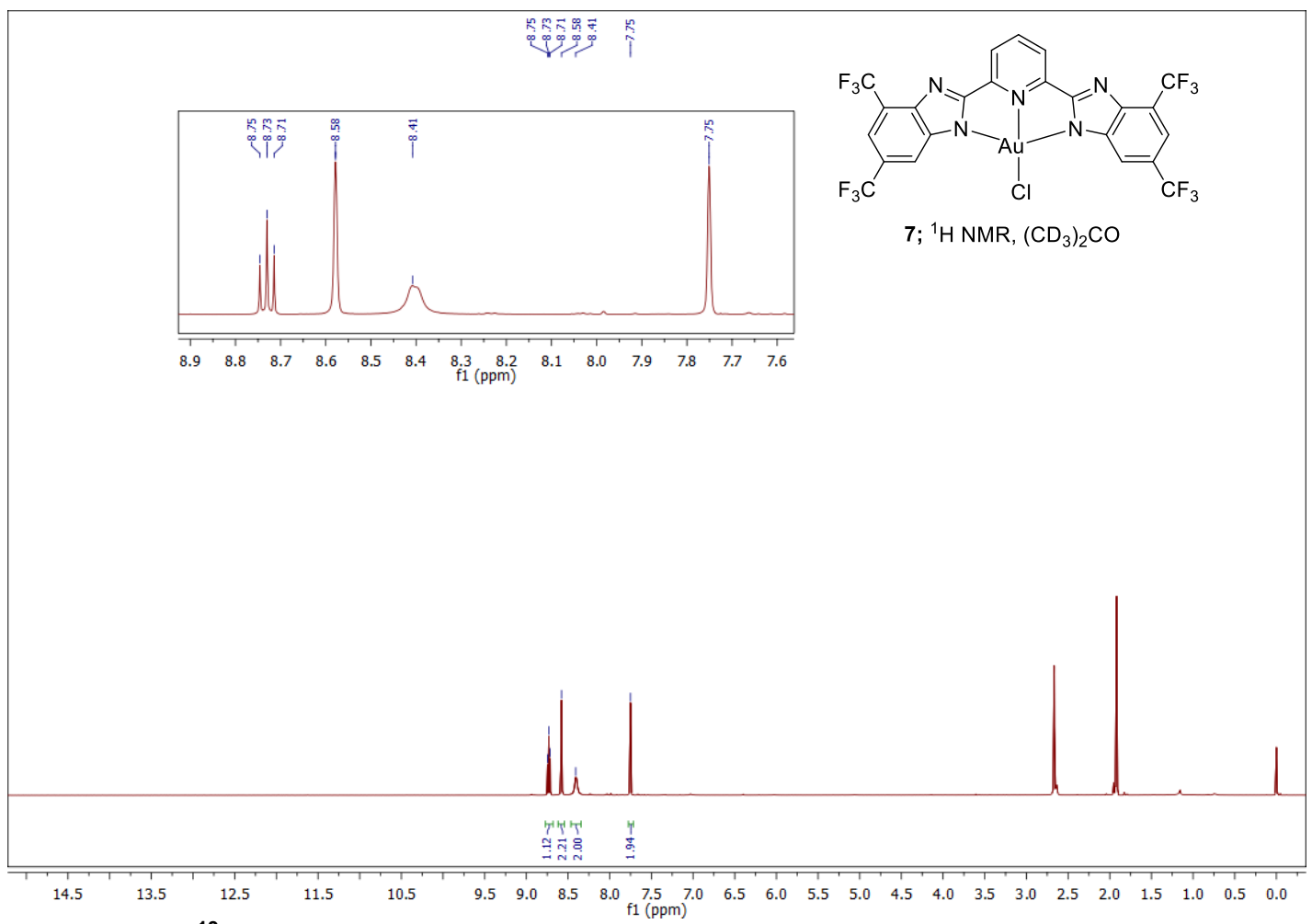

Figure S18: ${ }^{13} \mathrm{C}$ NMR of Complex 7

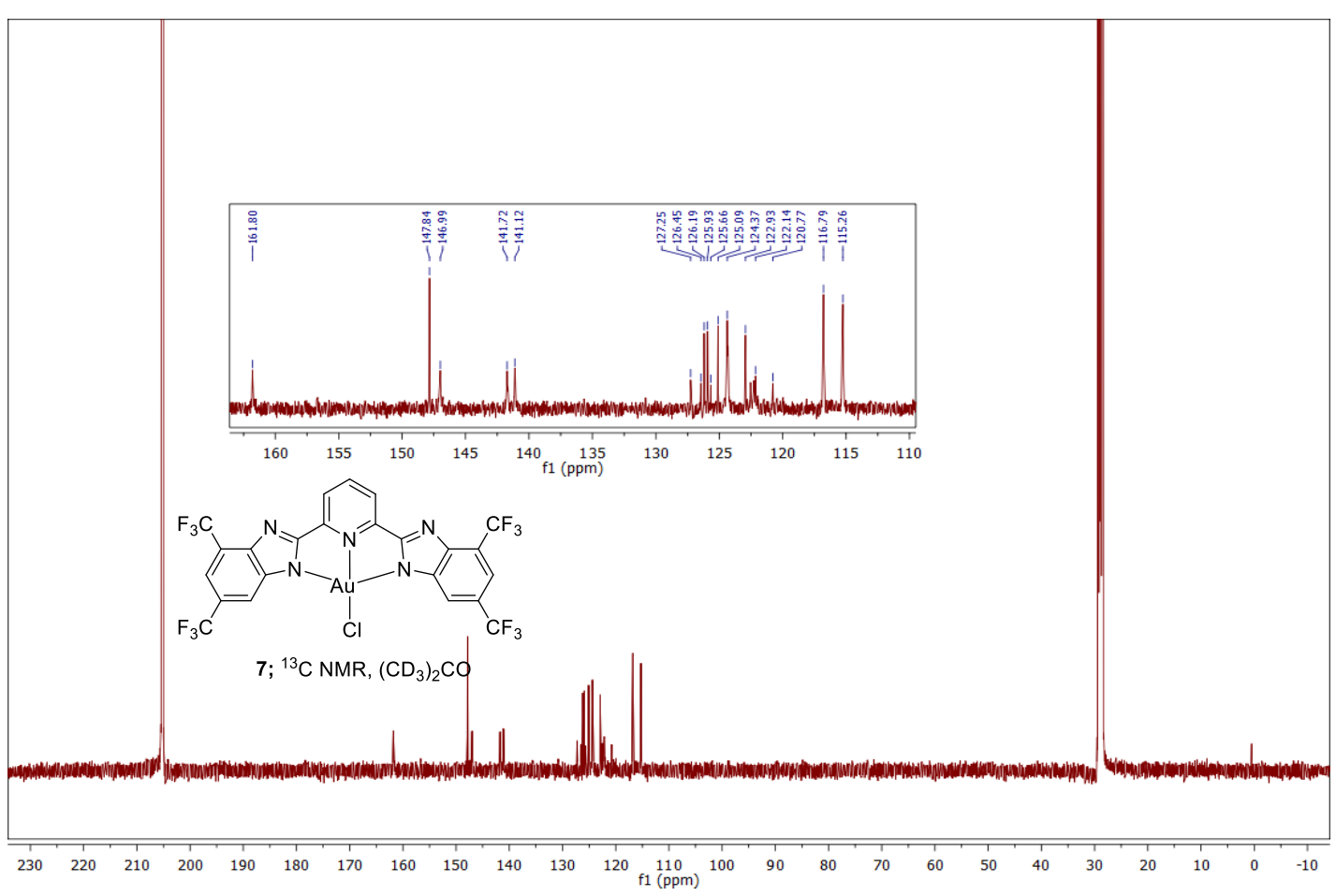


Figure S19: ${ }^{19} \mathrm{~F}$ NMR of Complex 7

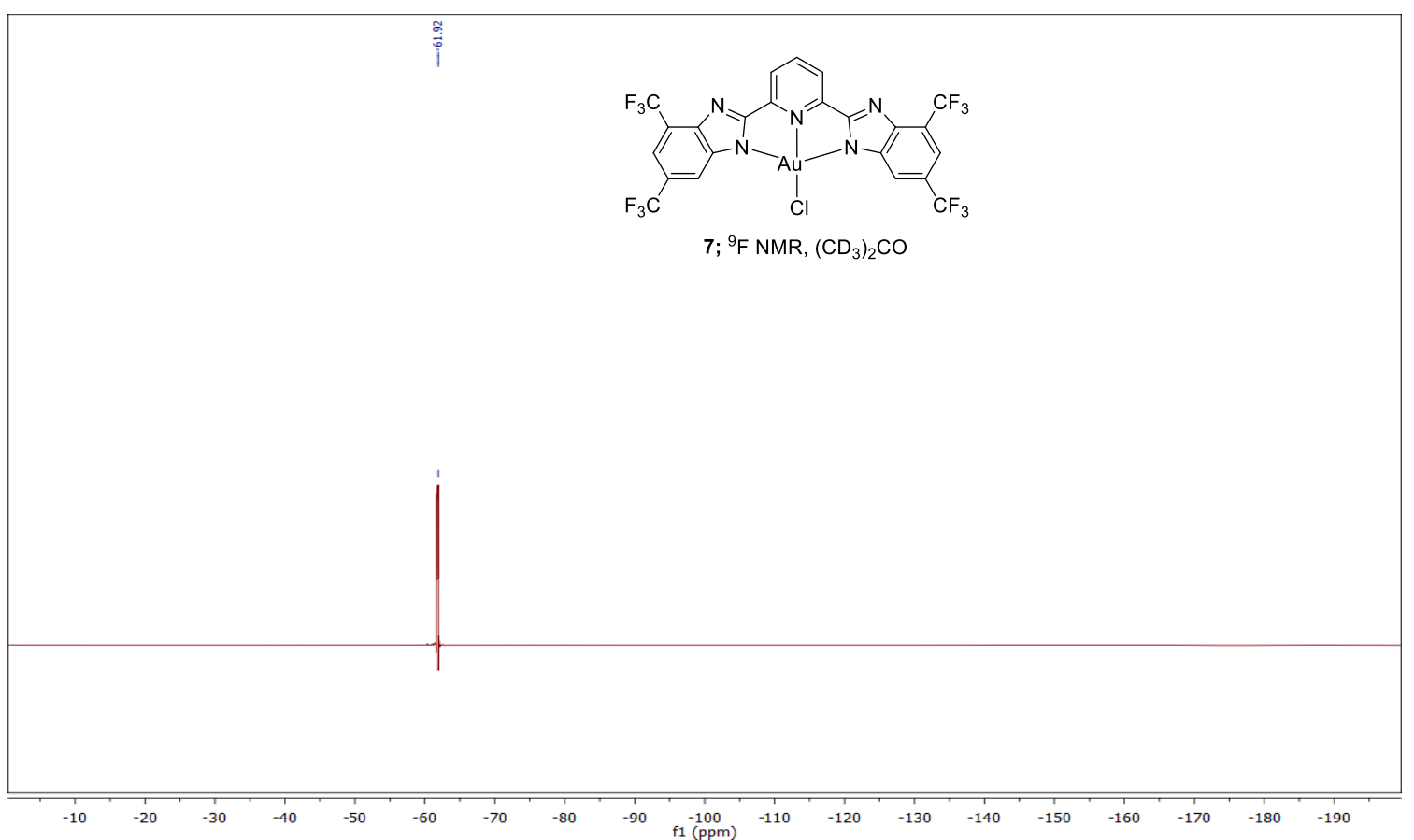


Figure S20: ${ }^{1} \mathrm{H}$ NMR of Complex 8 (triflate salt)

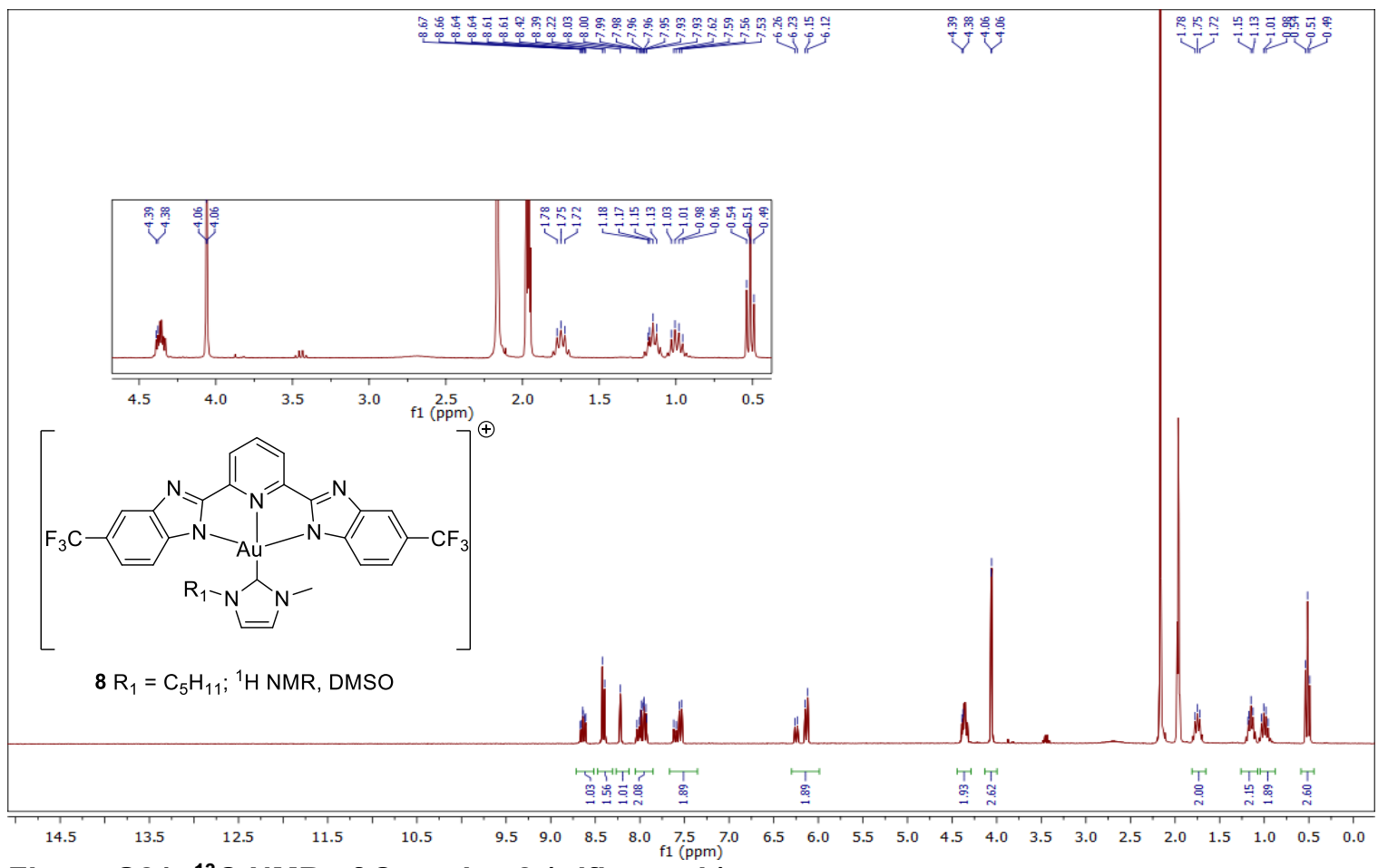

Figure S21: ${ }^{13} \mathrm{C}$ NMR of Complex 8 (triflate salt)

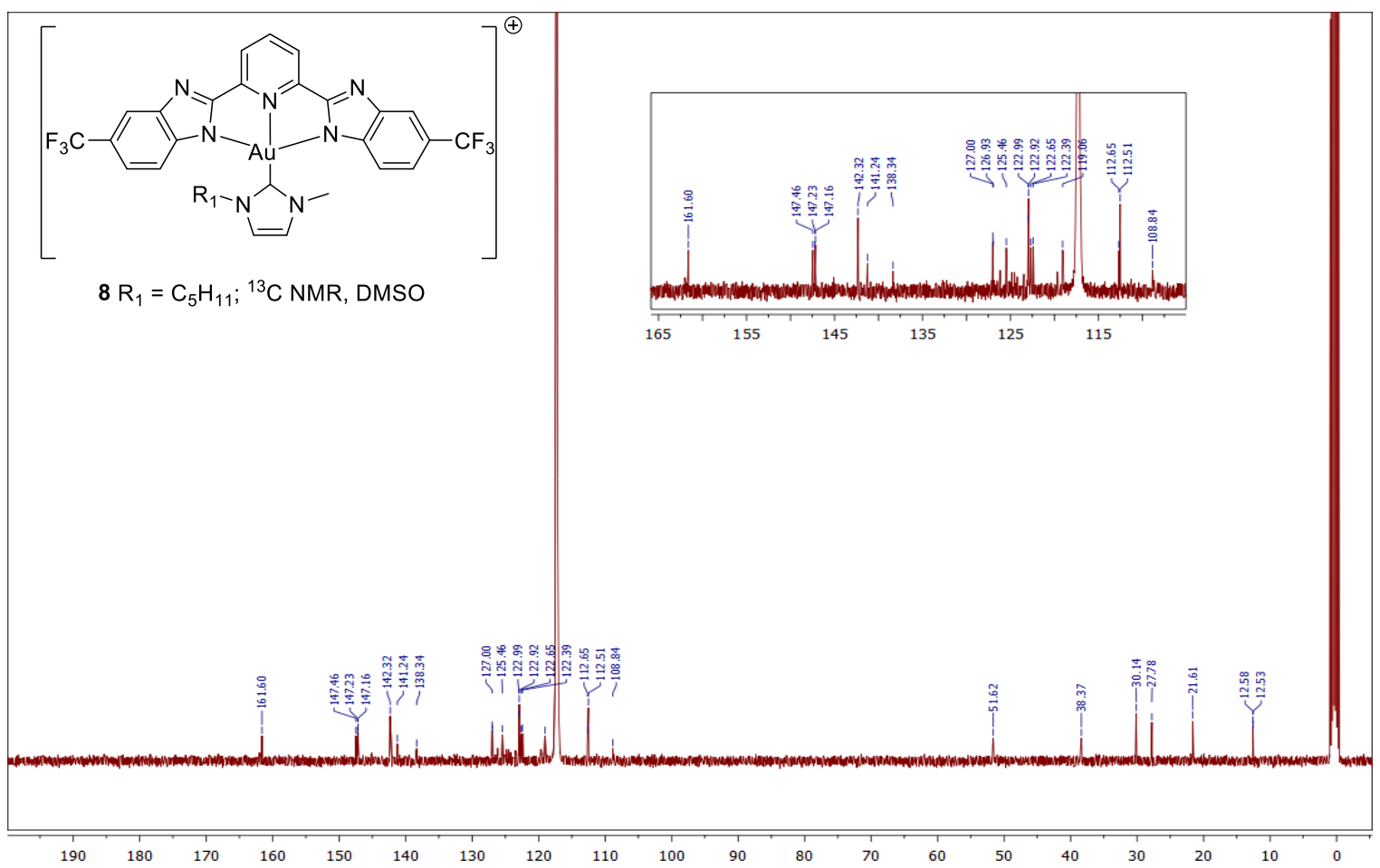


Figure S22: ${ }^{1} \mathrm{H}$ NMR of Complex 9 (triflate salt)

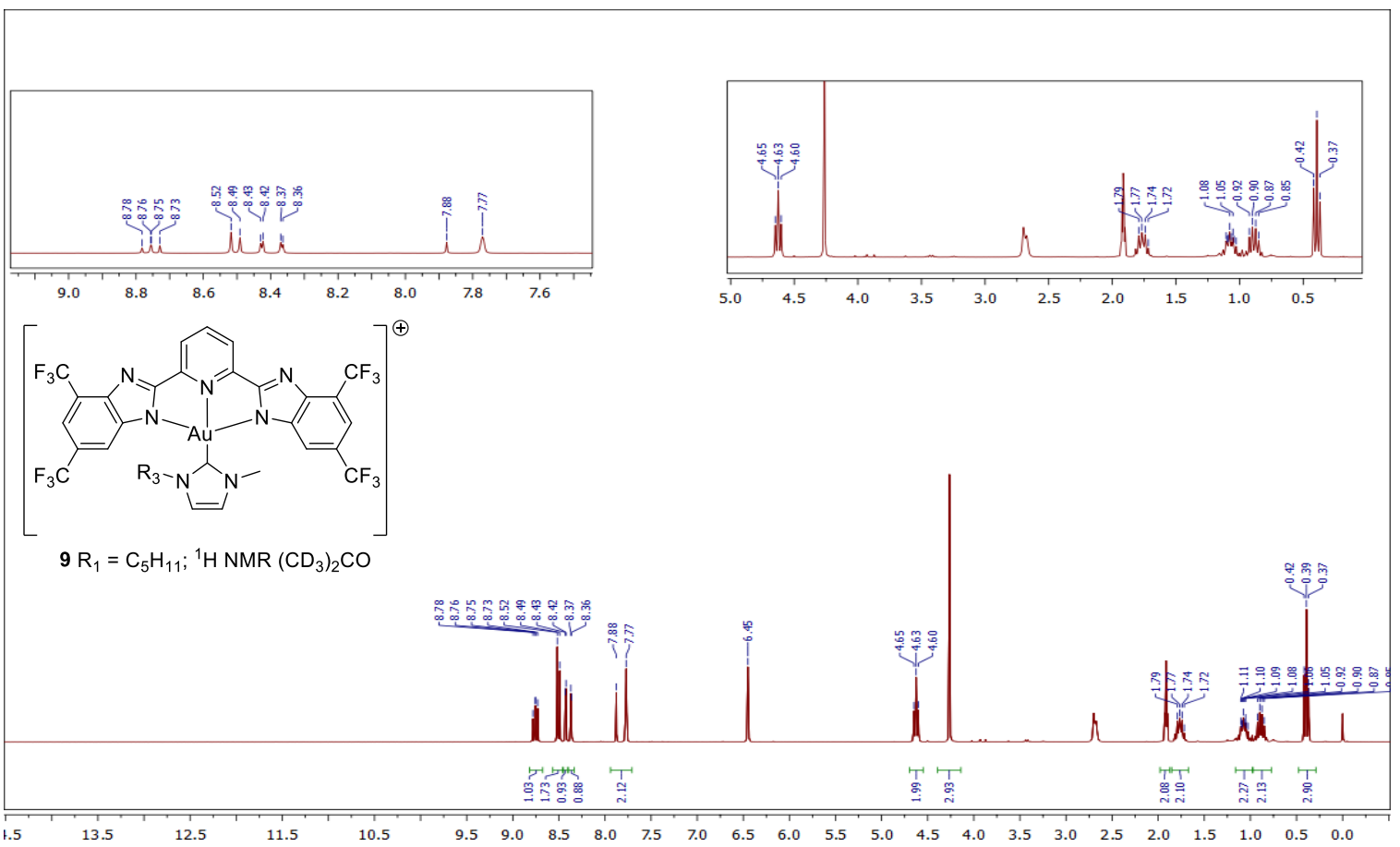

Figure S23: ${ }^{13} \mathrm{C}$ NMR of Complex 9 (triflate salt)

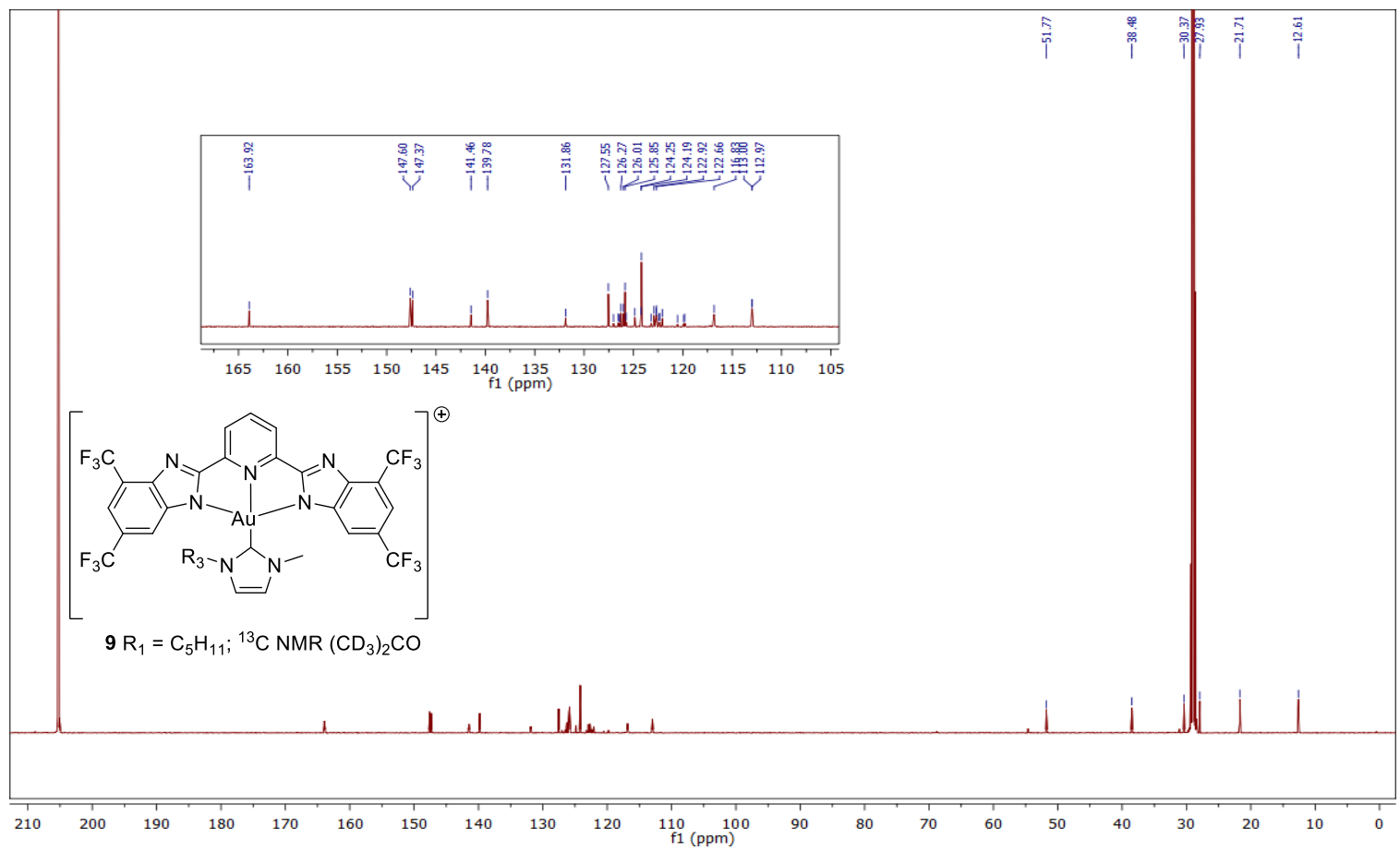


Figure S24: ${ }^{1} \mathrm{H}$ NMR of 1-pentyl-3-methyl imidazolium bromide

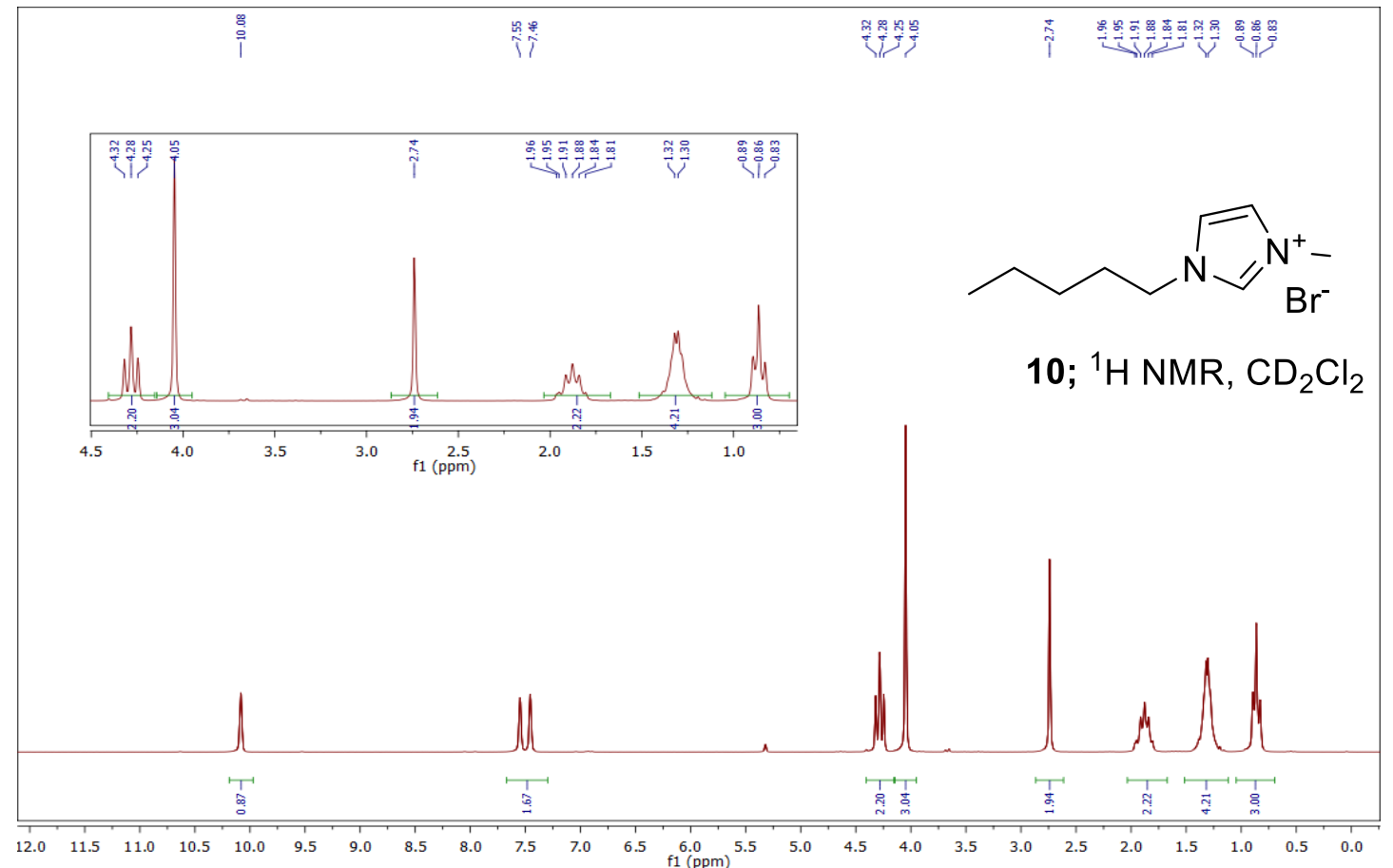

Figure S25: ${ }^{13} \mathrm{C}$ NMR of 1-pentyl-3-methyl imidazolium bromide

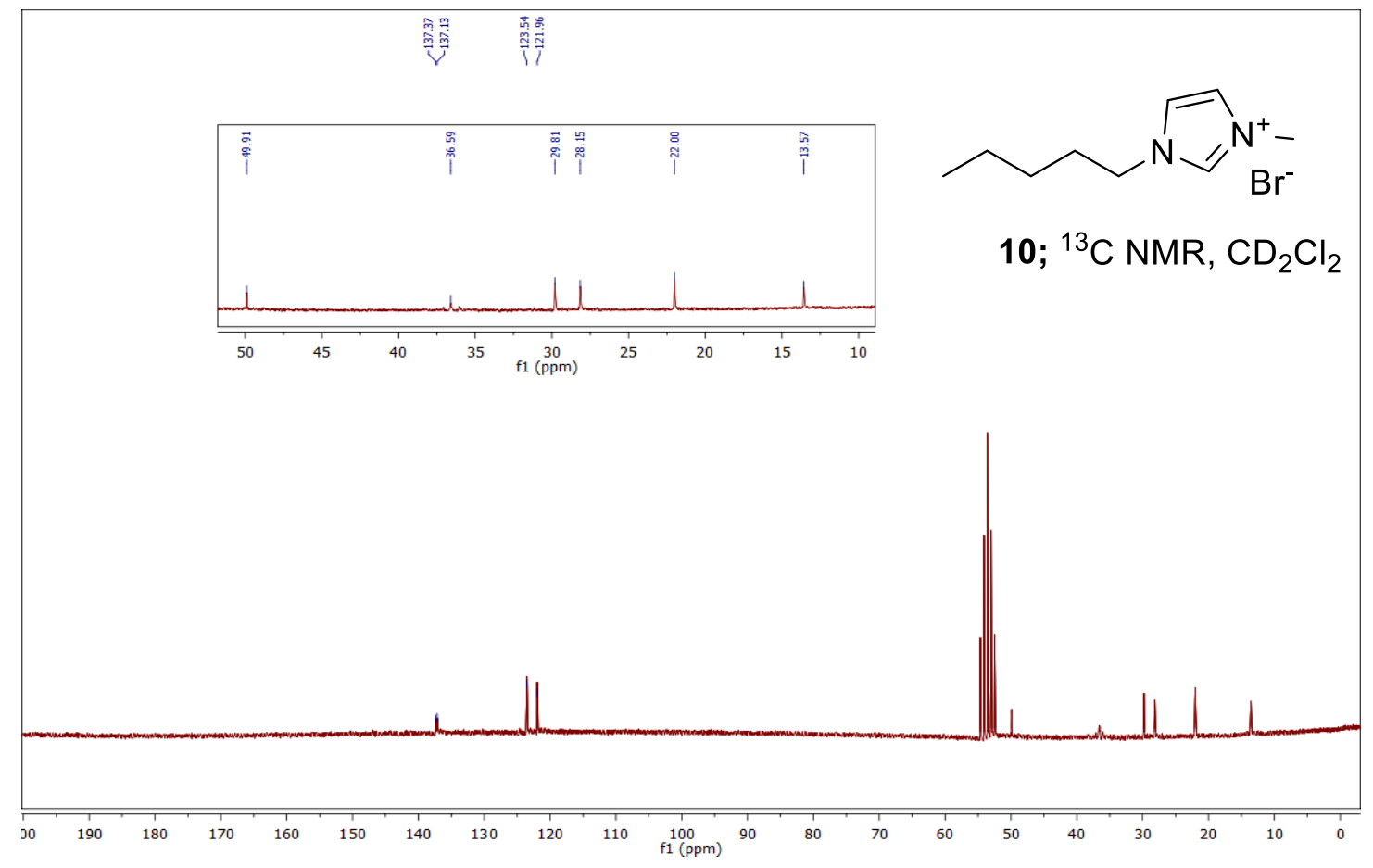


Figure S26: ${ }^{1} \mathrm{H}$ NMR of (1-methyl-3-pentyl-1,3-dihydro-2H-imidazol-2-ylidene)silver I bromide

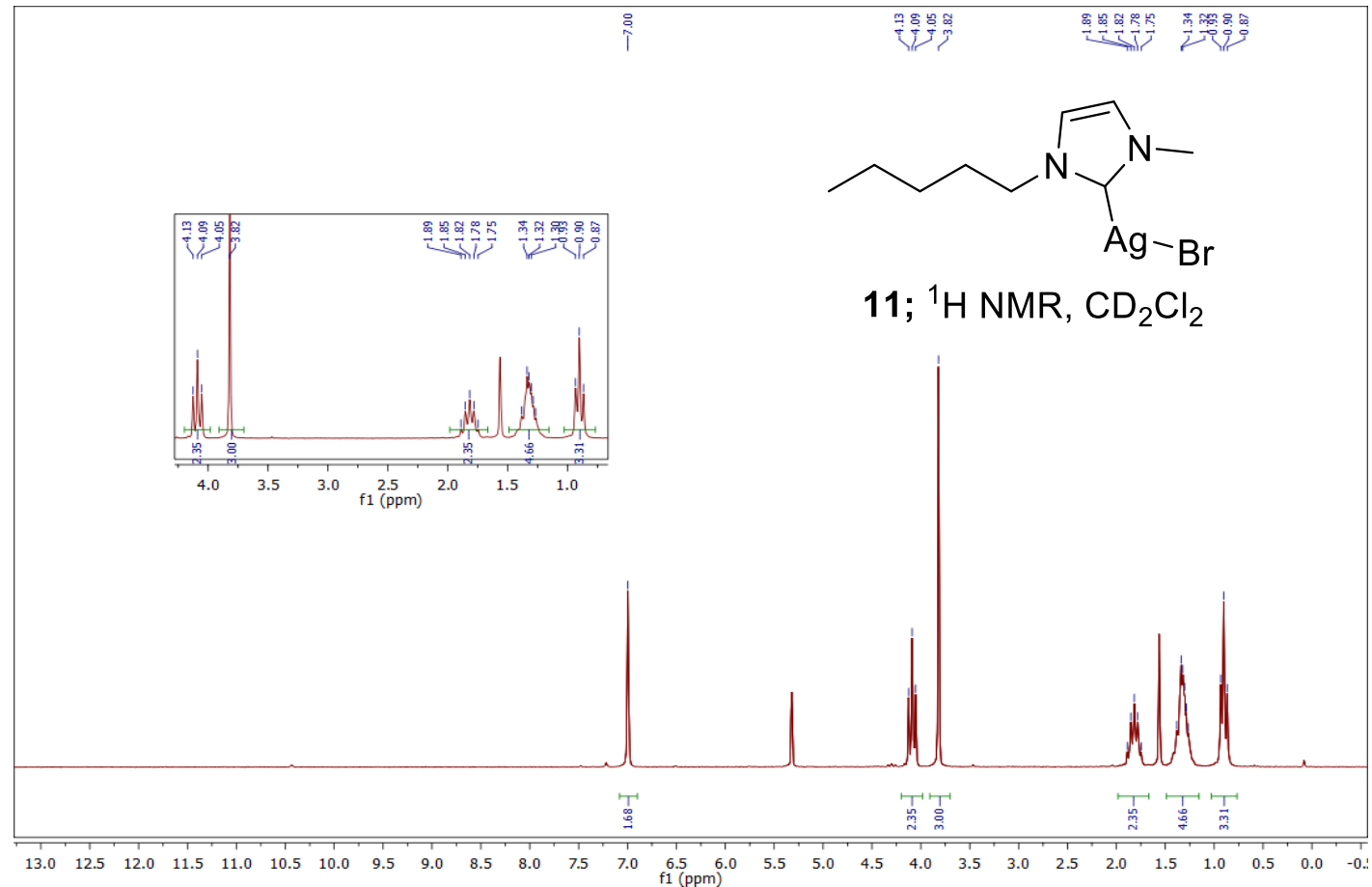

Figure S27: ${ }^{1} \mathrm{H}$ NMR of (1-methyl-3-pentyl-1,3-dihydro-2H-imidazol-2-ylidene)silver I bromide

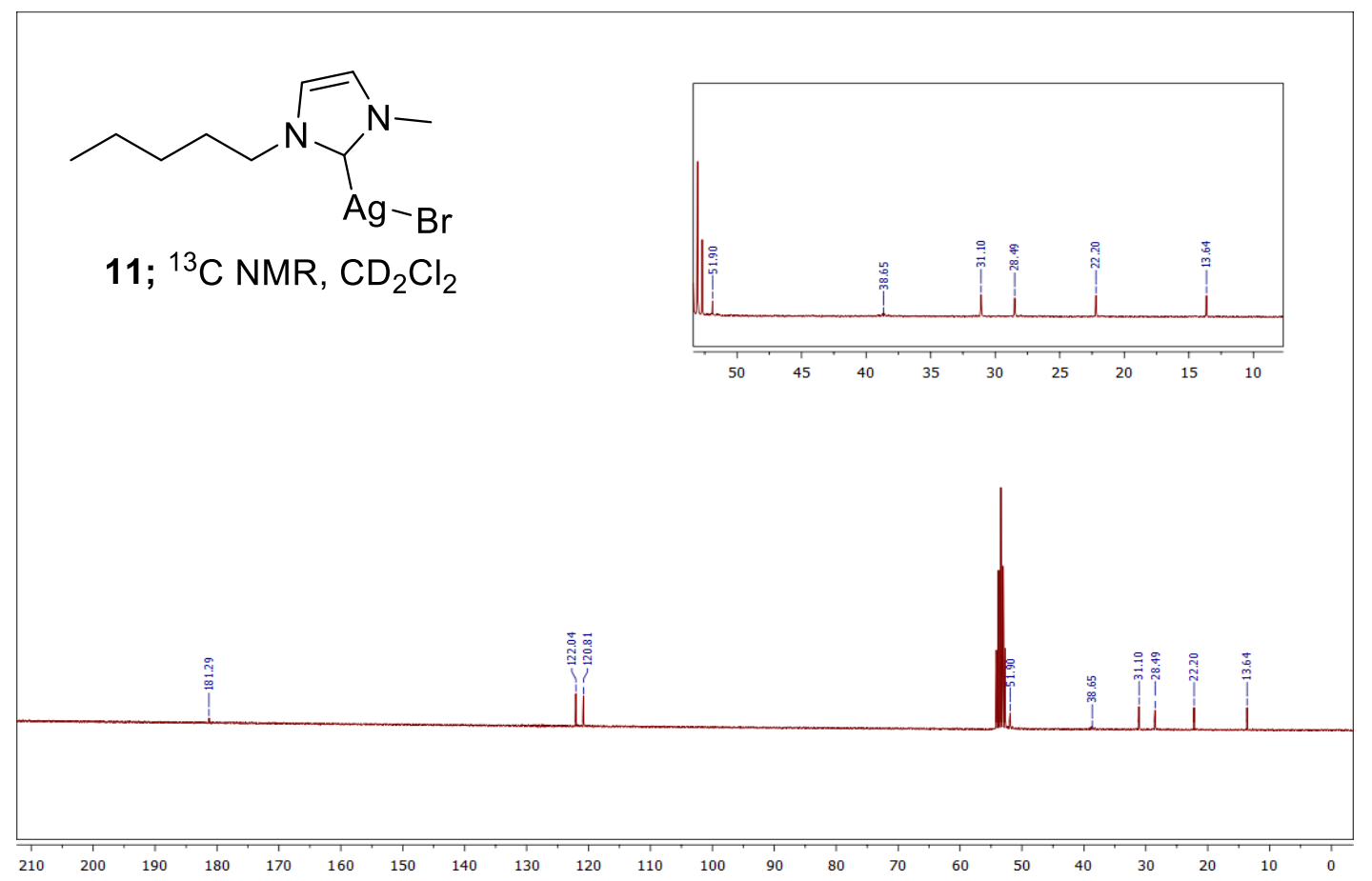




\section{S4: Mass spectra}

Figure S28: Low resolution mass spectra of 1

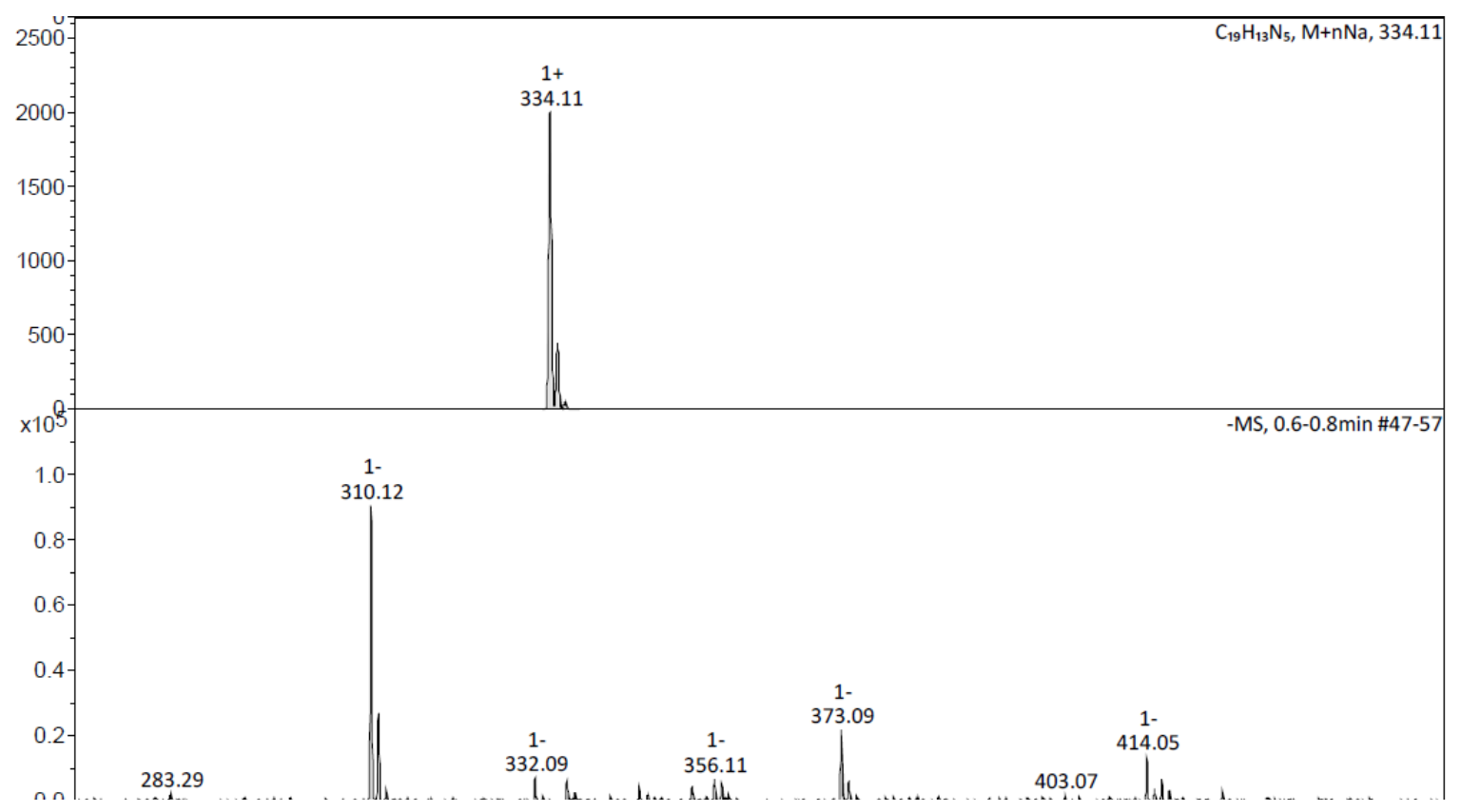

Figure S29: High resolution mass spectrum of 1

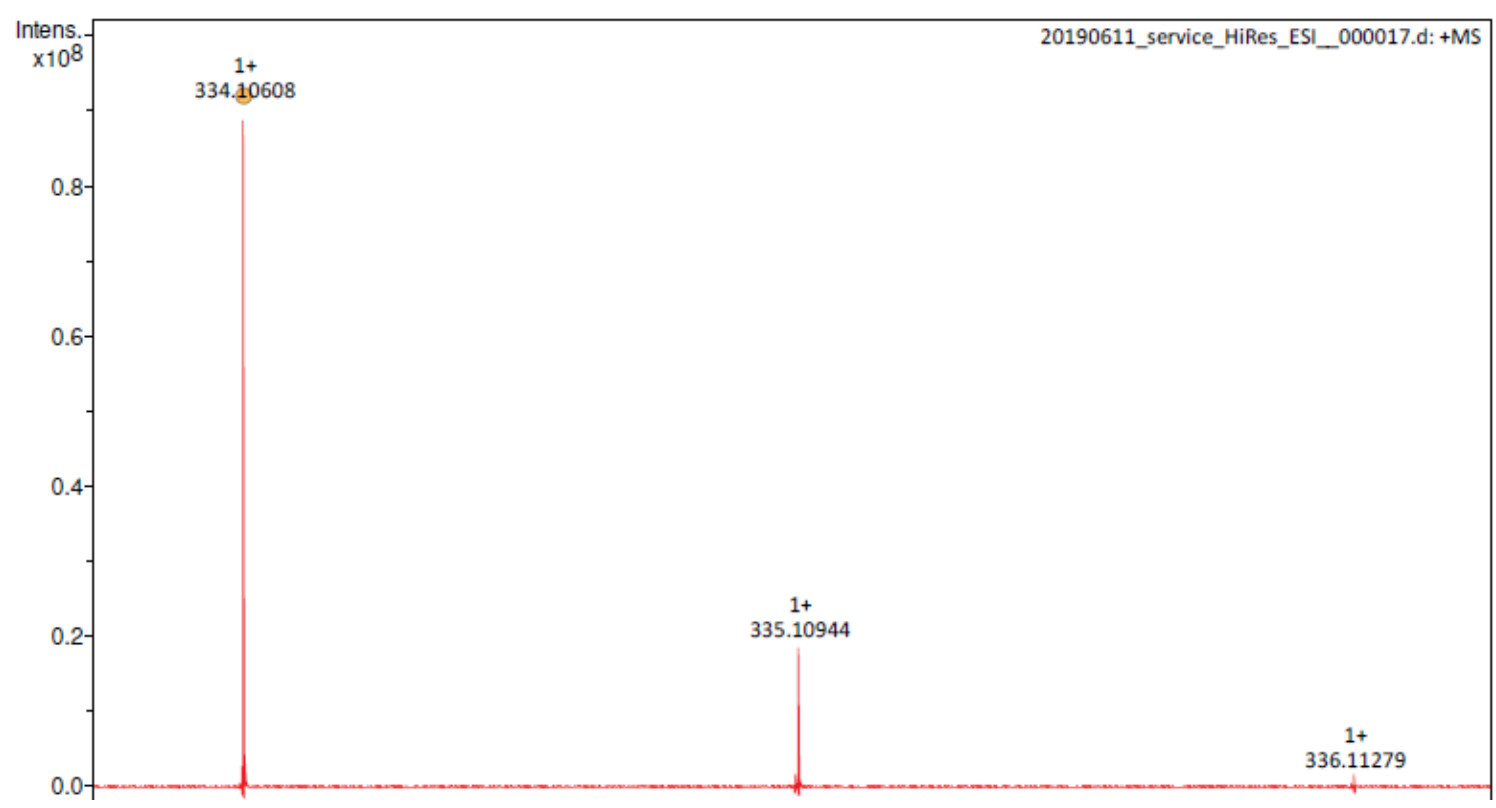


Figure S30: Low resolution mass spectra of 2

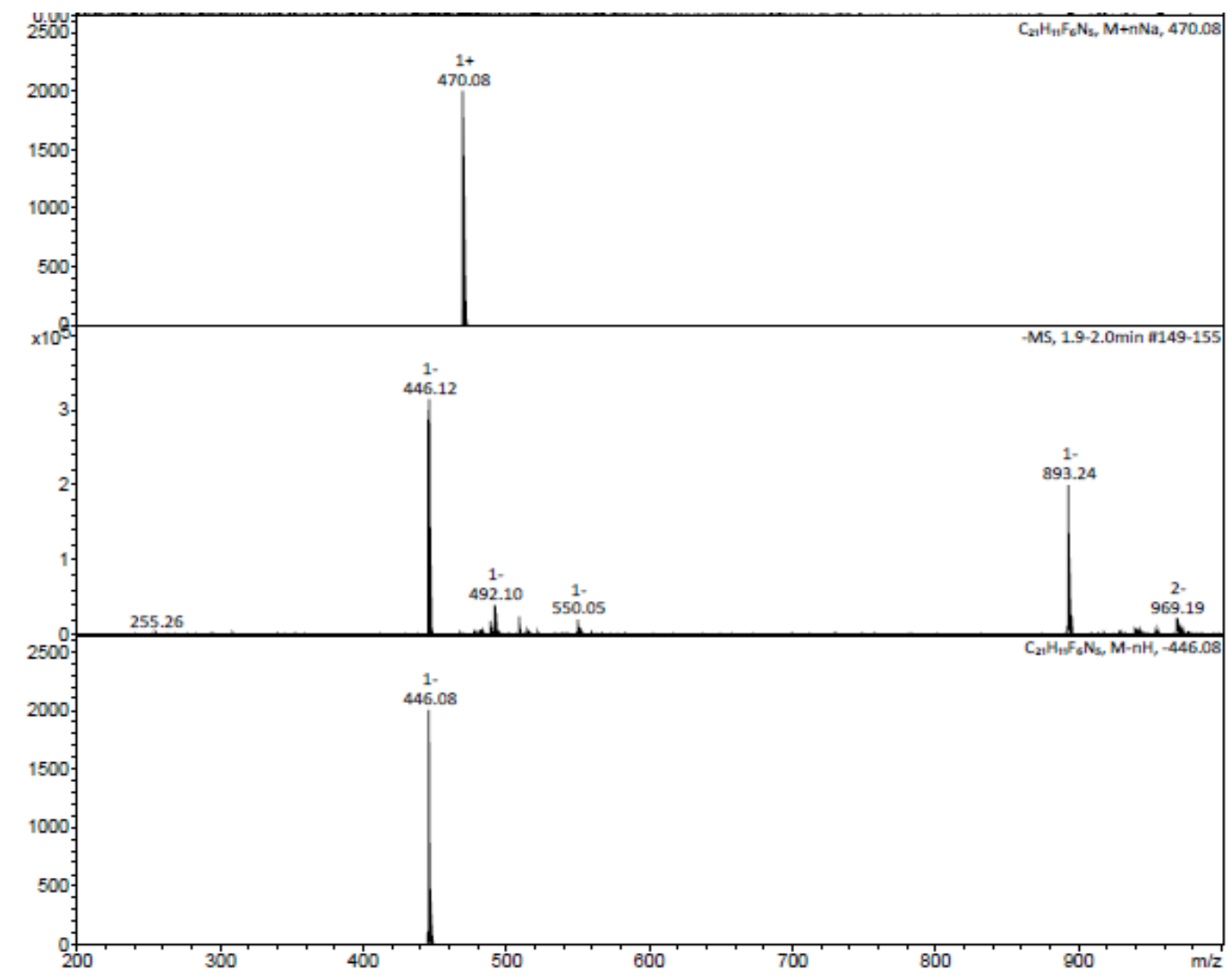

Figure S31: High resolution mass spectrum of 2

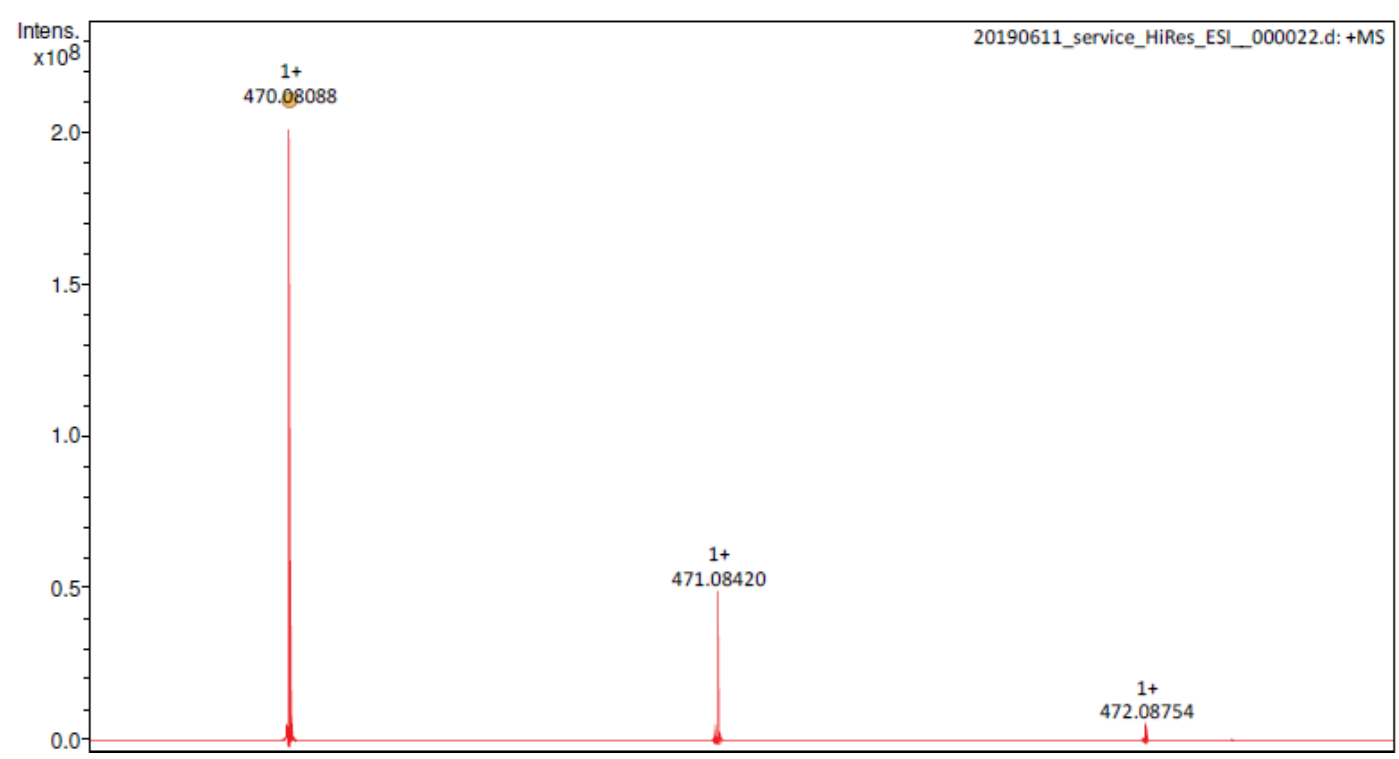


Figure S32: Low resoltion mass spectra of 3

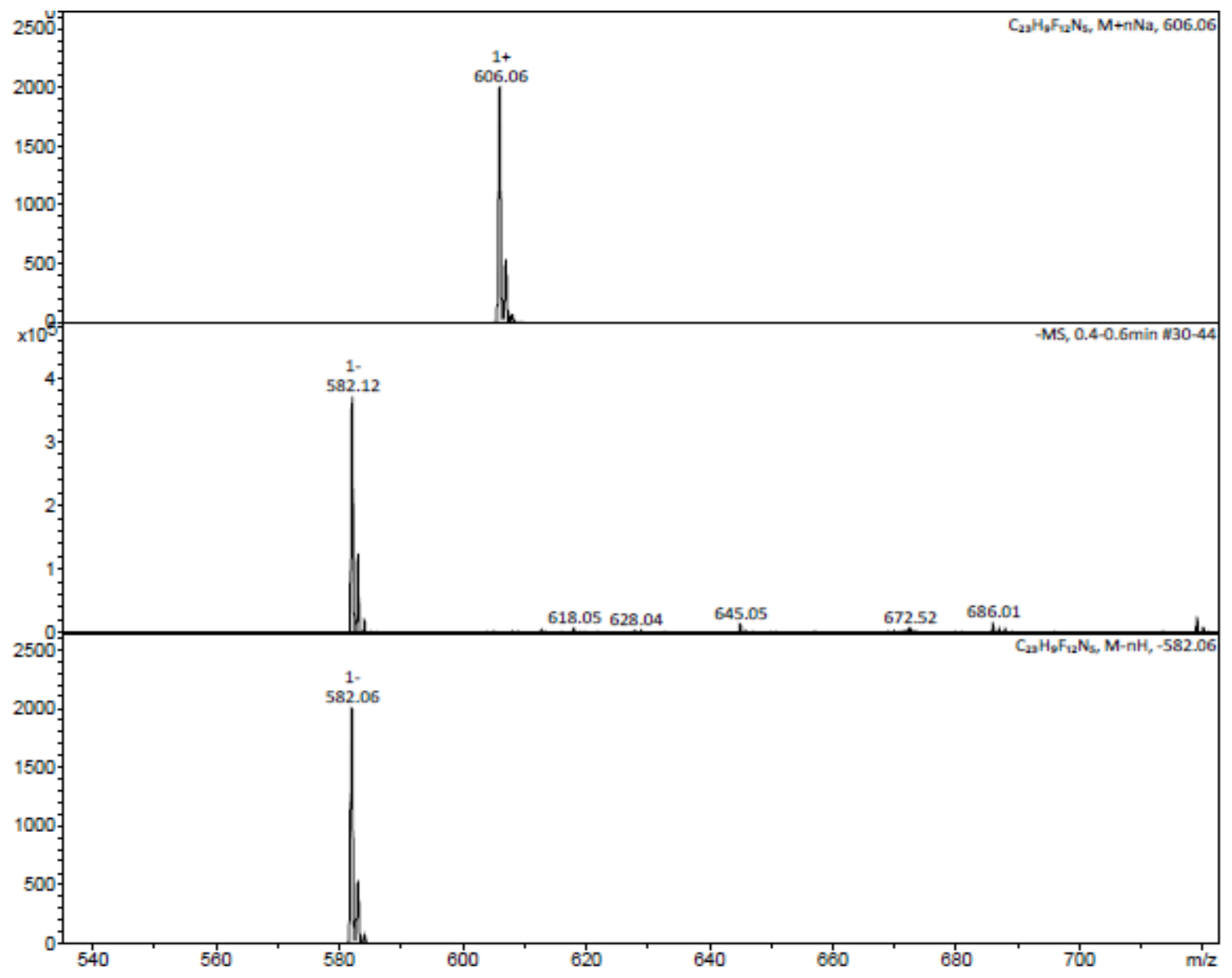

Figure S33: High resolution mass spectrum of 3

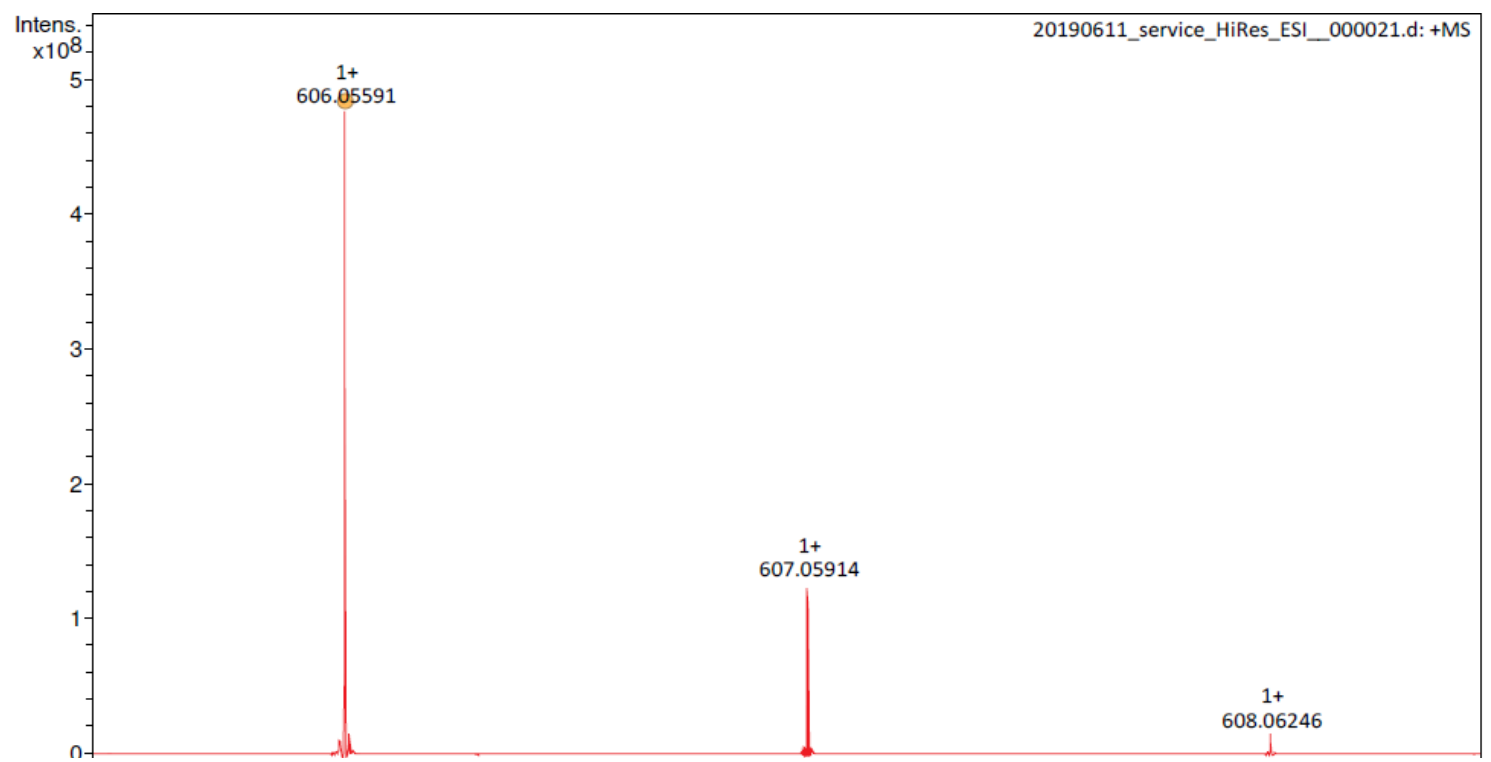


Figure S34: Low resolution mass spectra of 4

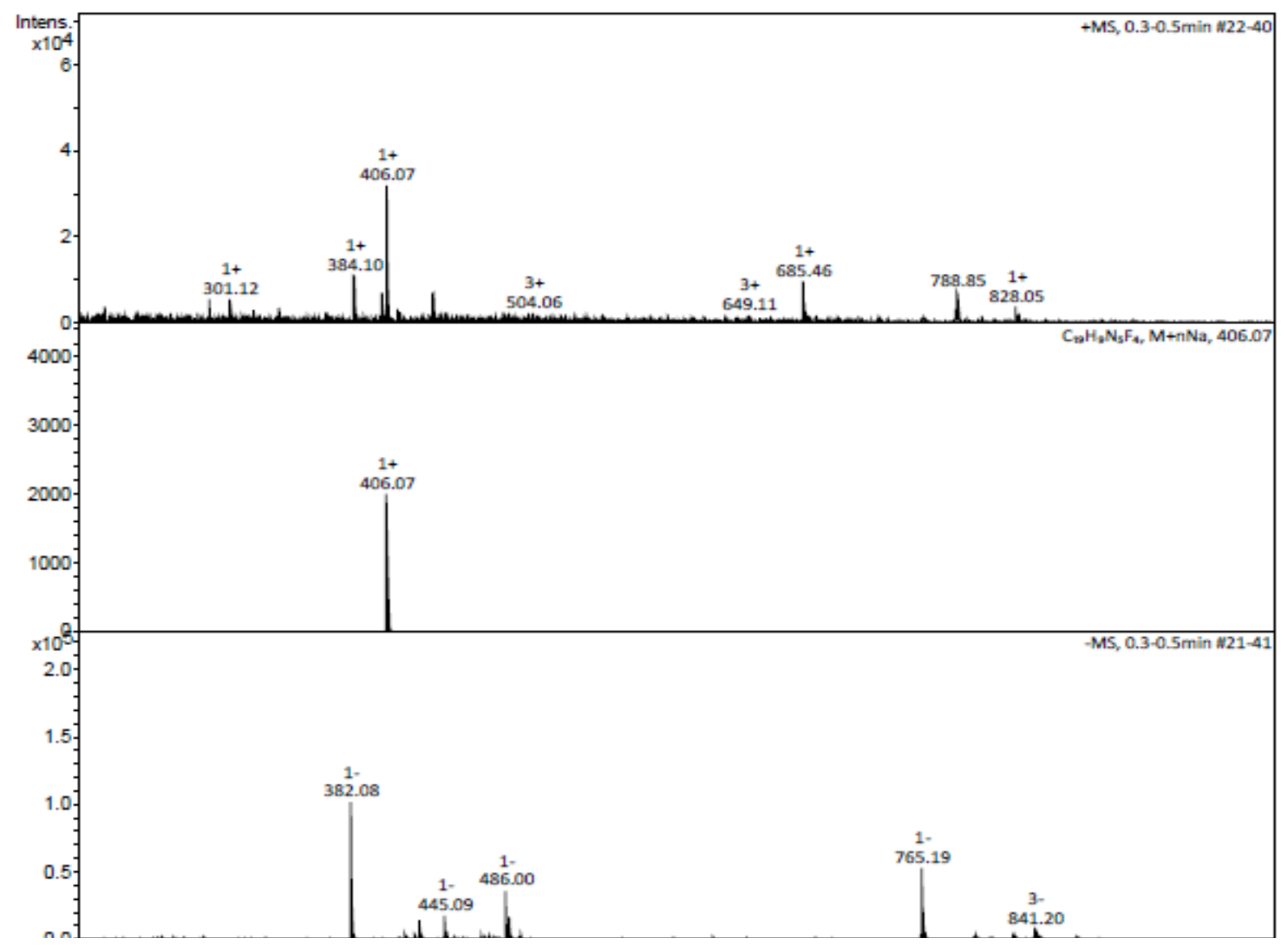

Figure S35: High resolution mass spectrum of 4

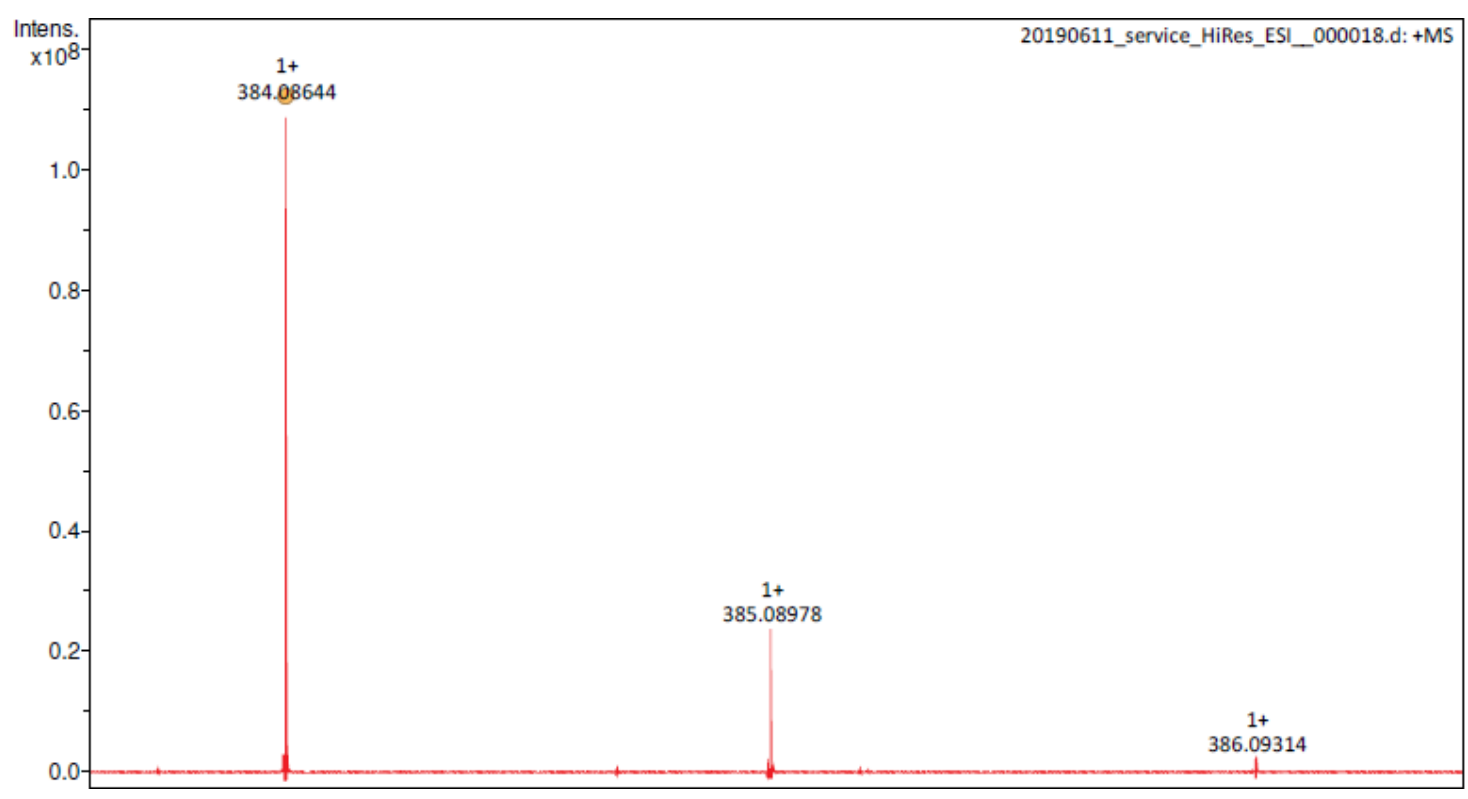


Figure S36: Low resolution mass spectra of 5

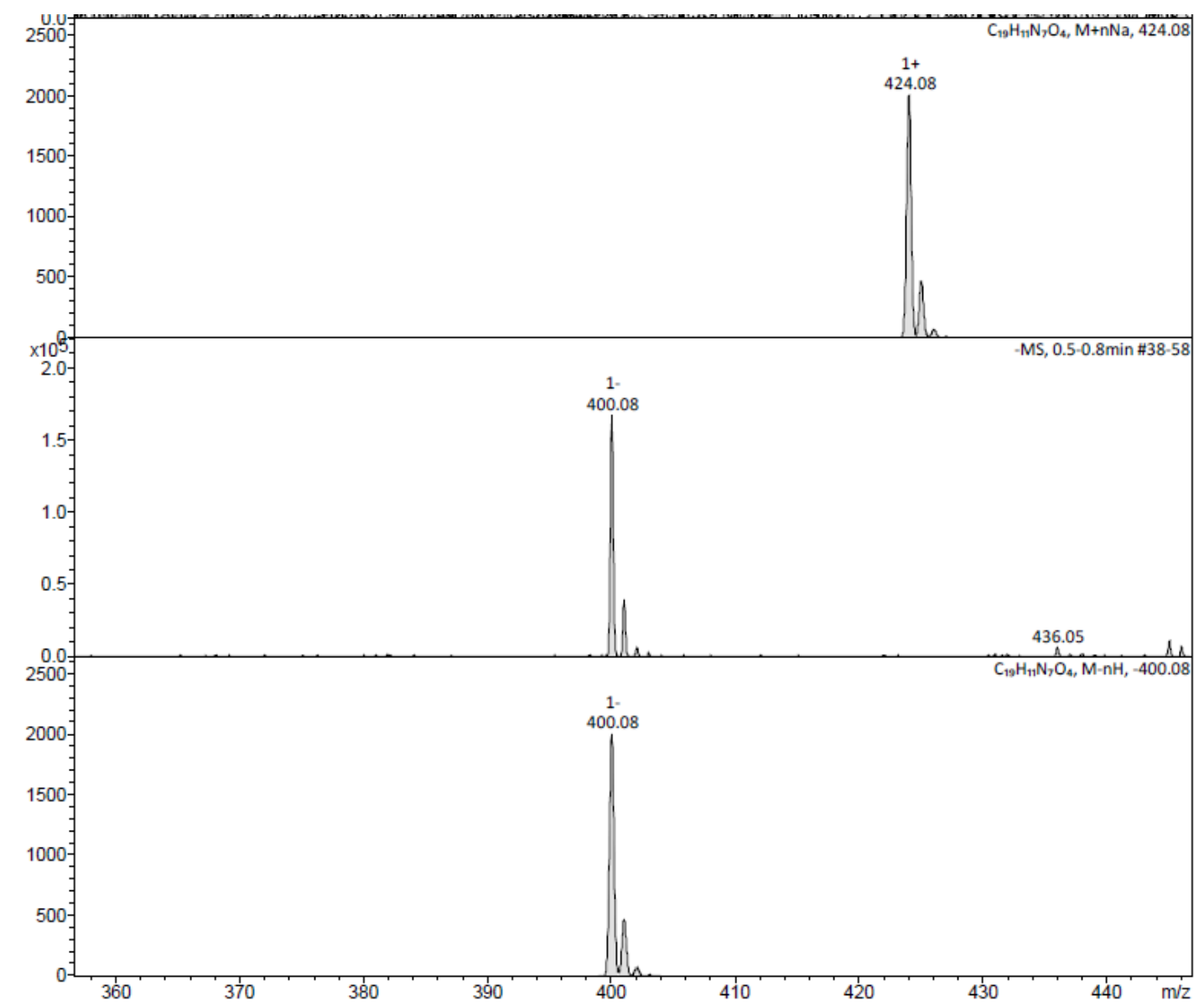

Figure S37: High resolution mass spectrum of 5

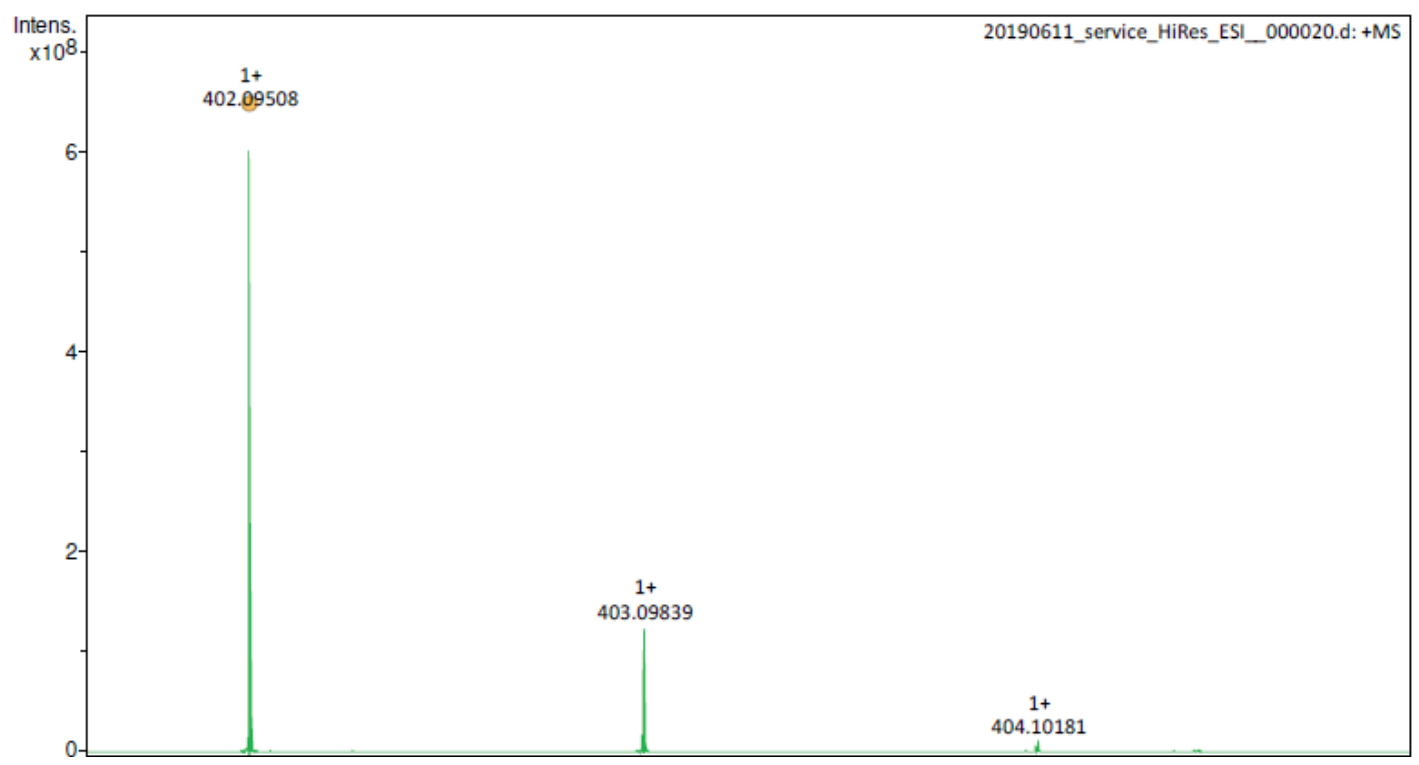


Figure S38: Low and high resolution mass spectra of complex 6

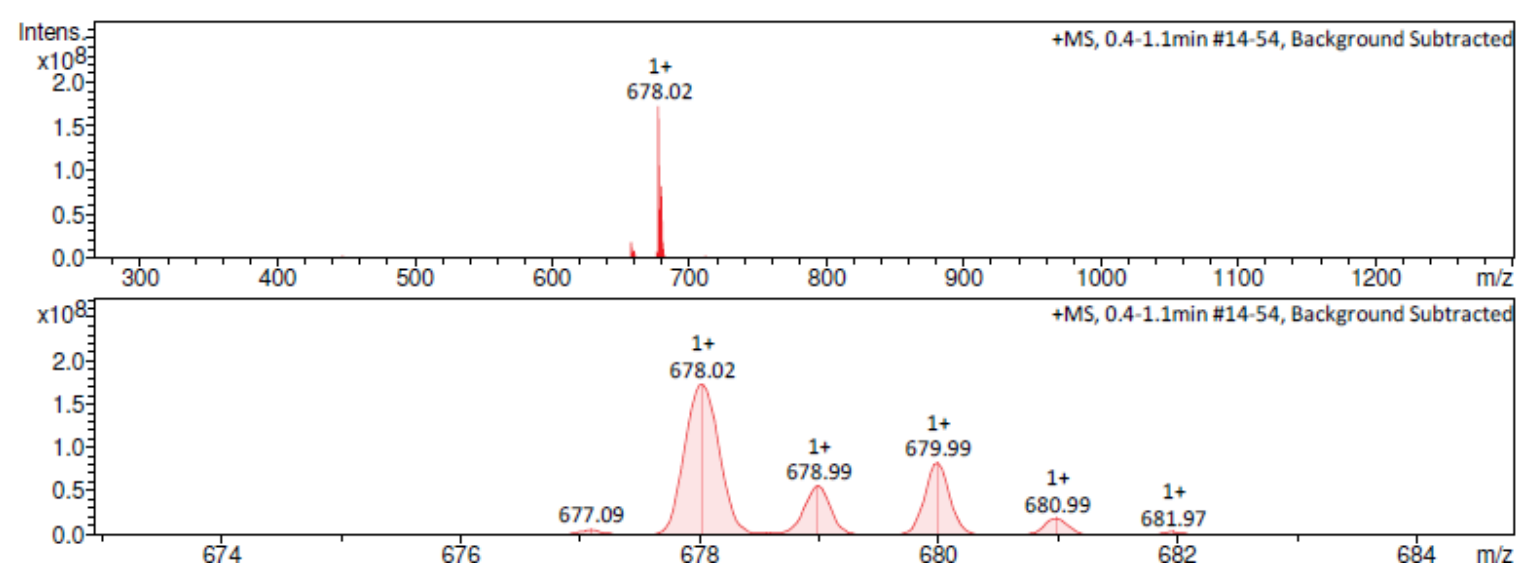

Figure S39: High resolution mass spectrum of complex 6

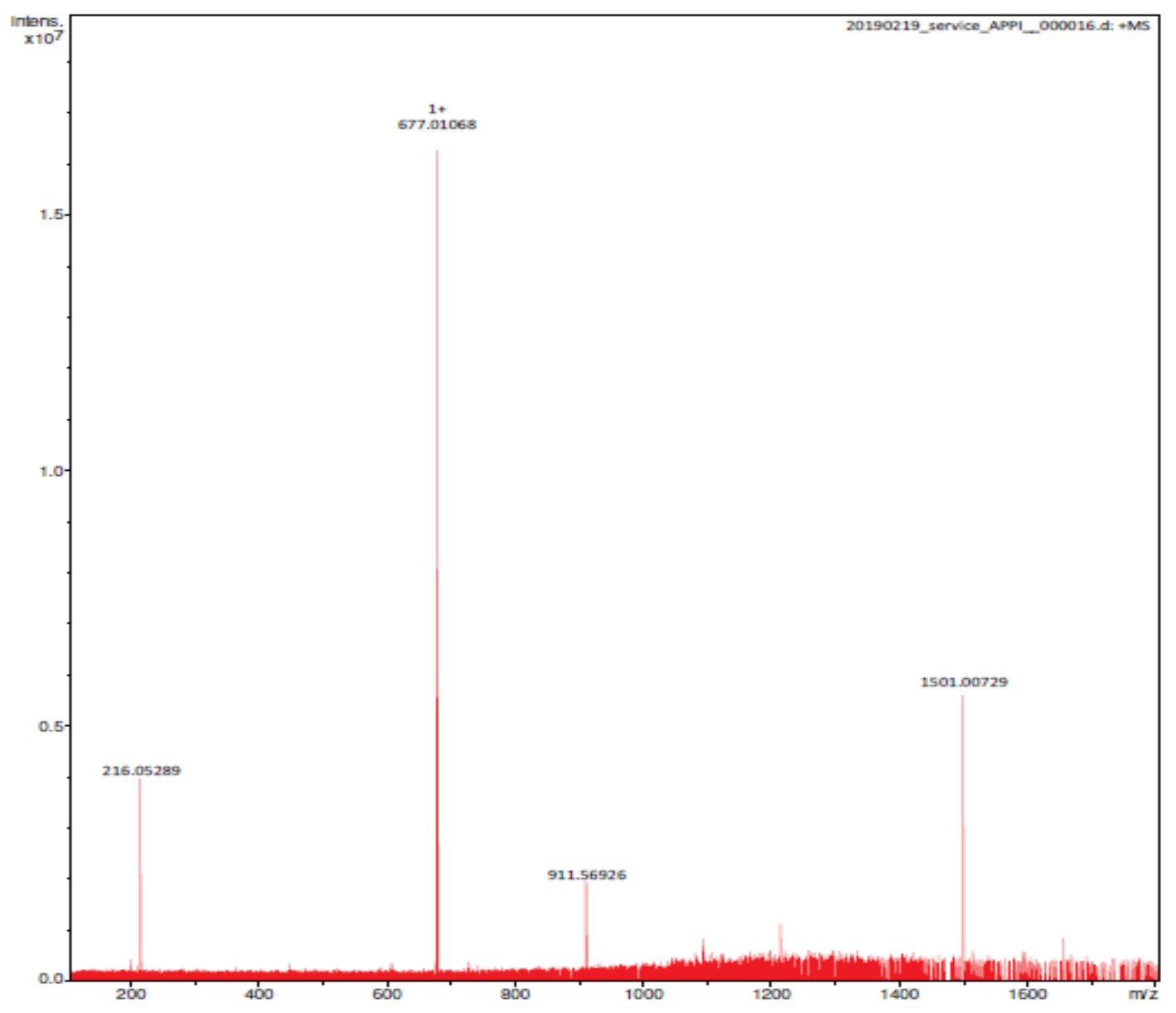


Figure S40: Low resolution mass spectra of complex 7

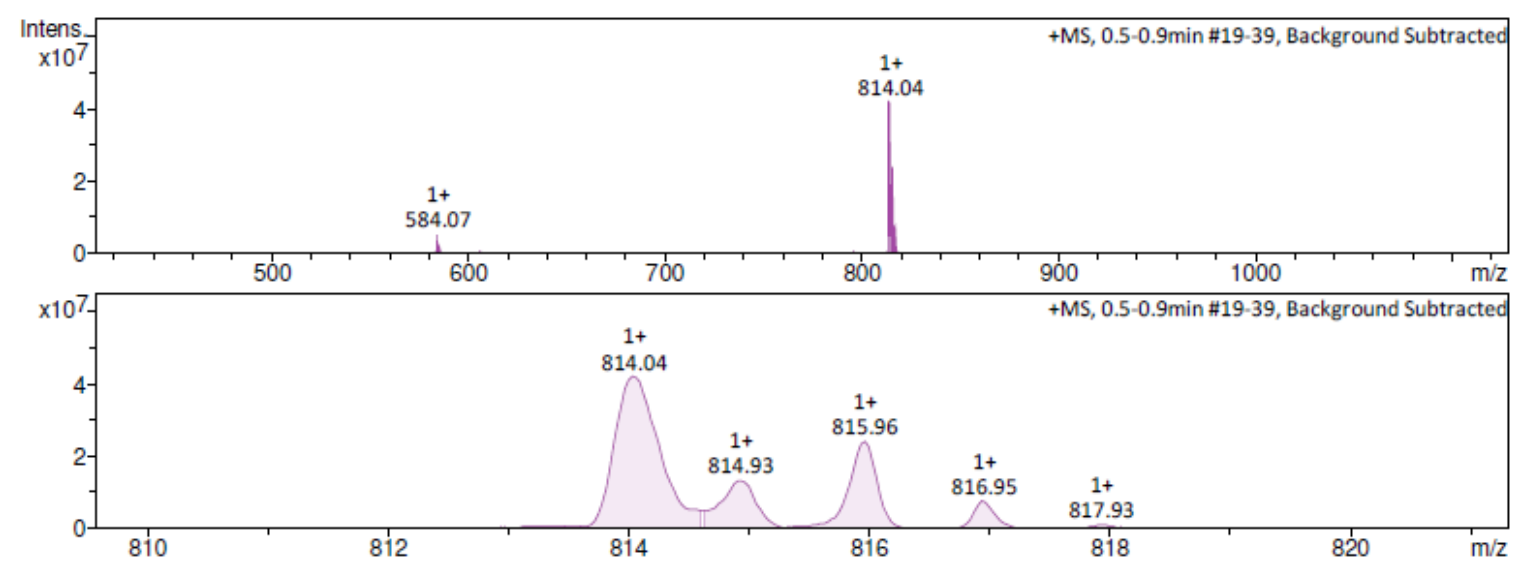

Figure S41: High resolution mass spectrum of complex 7

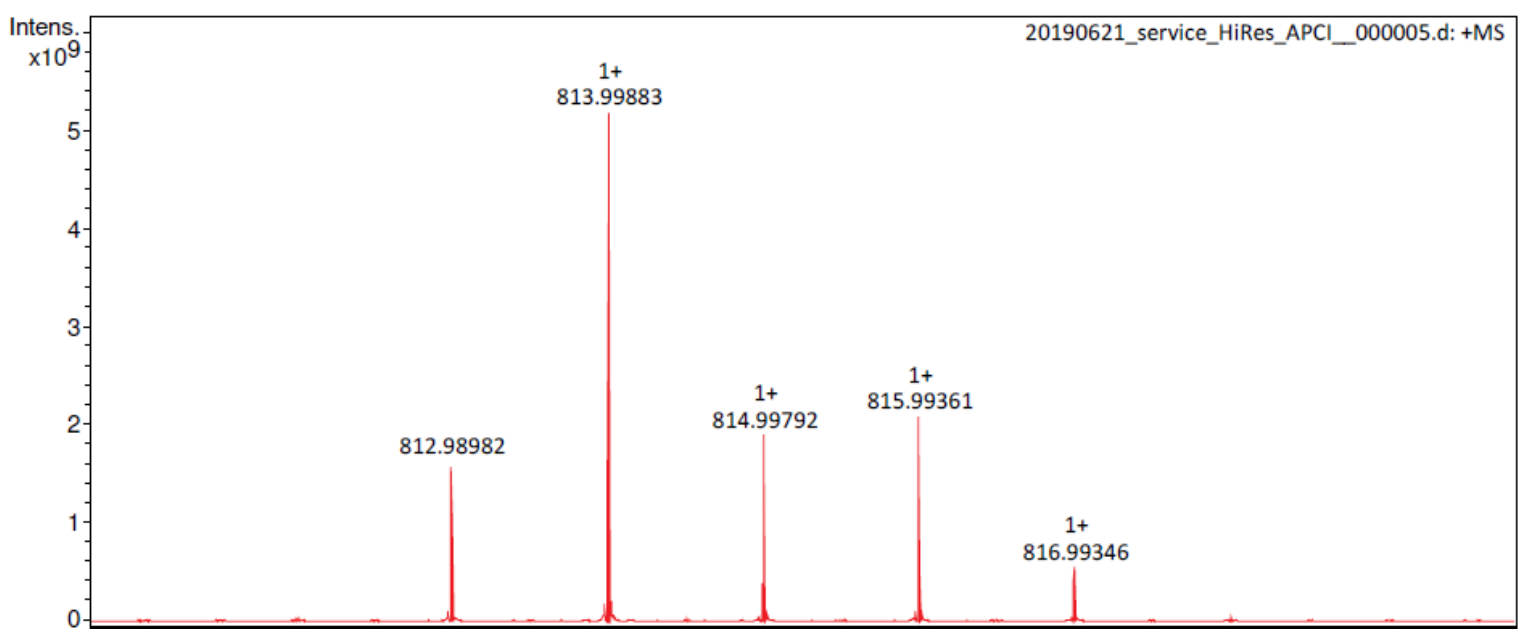


Figure S42: Low resolution mass spectra of complex 8
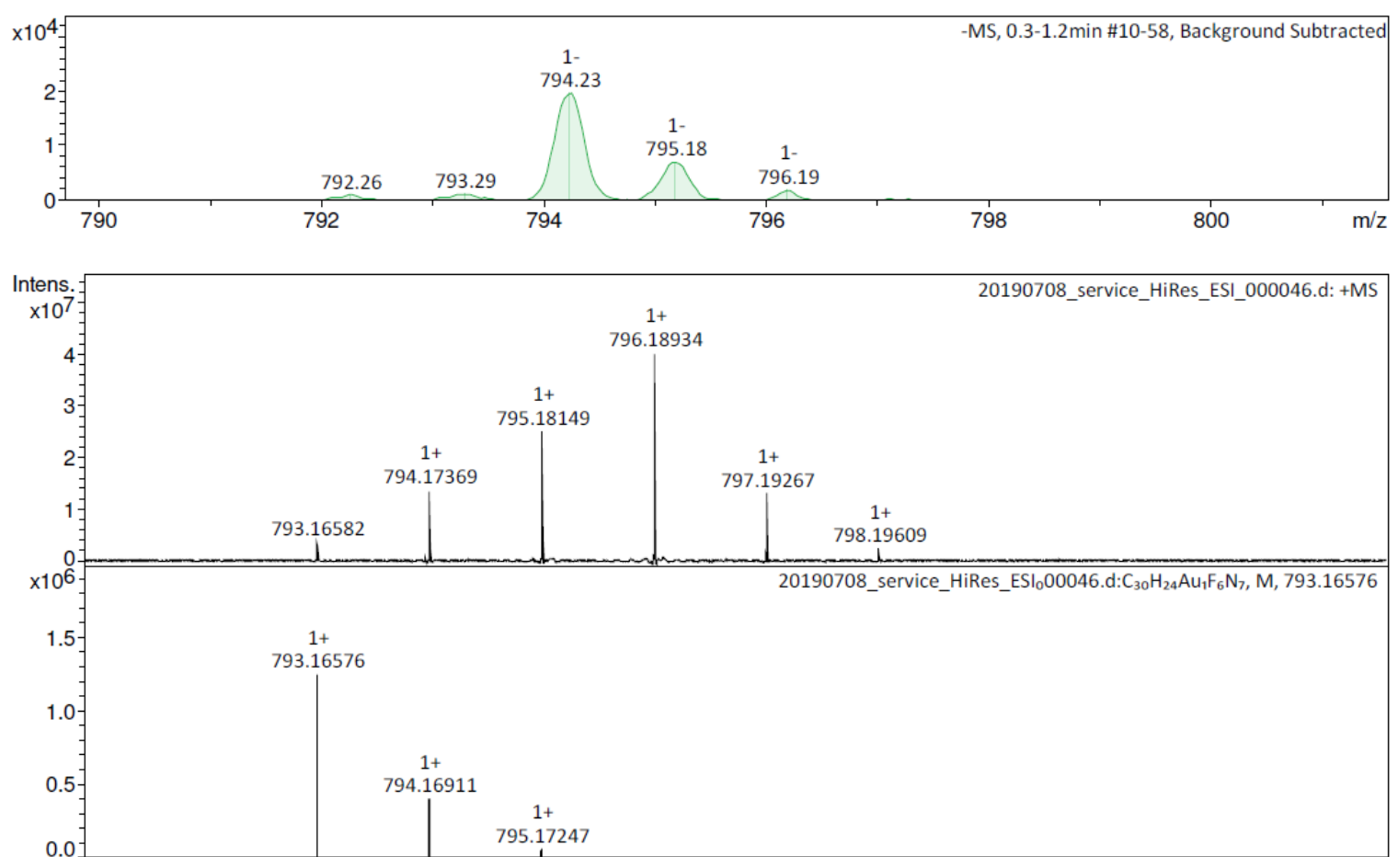

Figure S43: High resolution mass spectra of complex 8
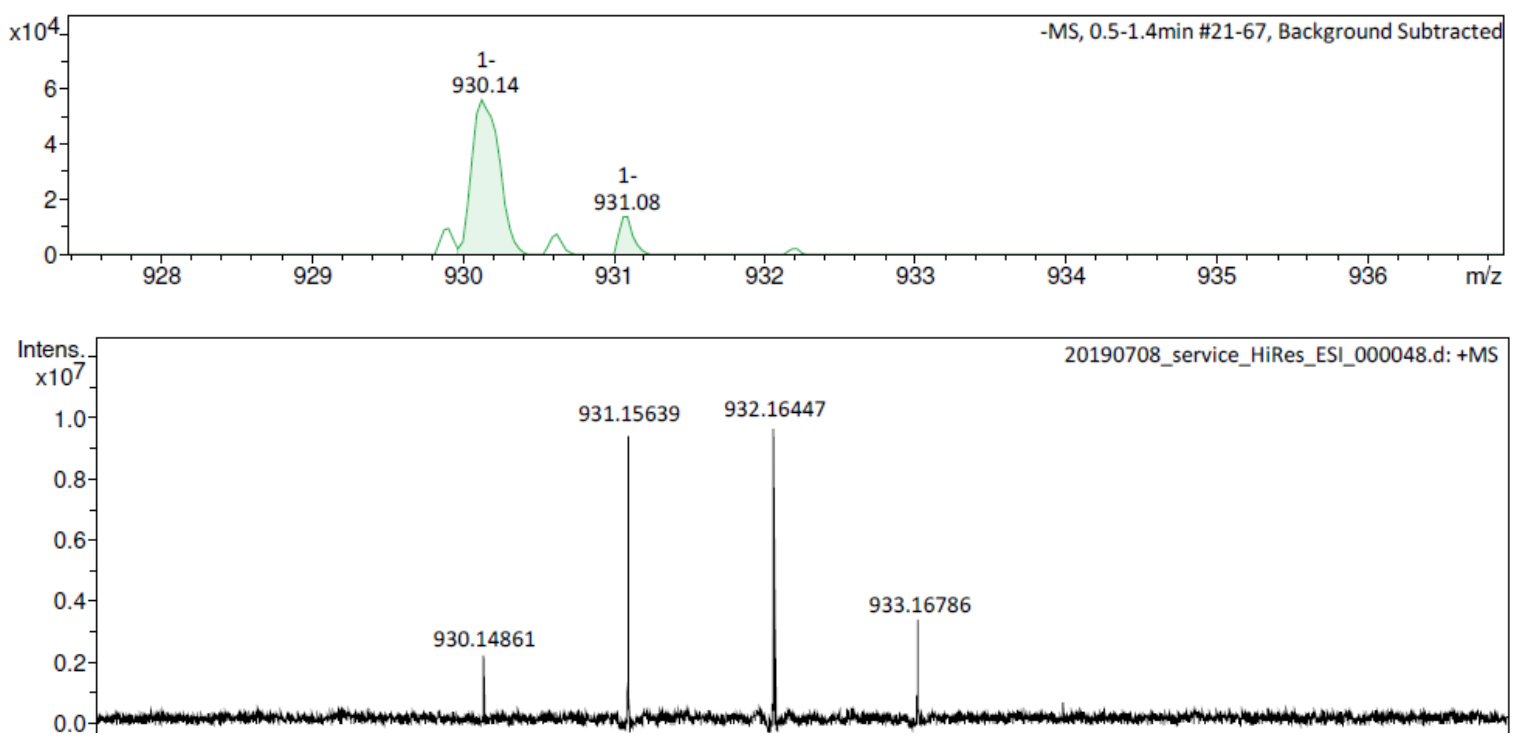


\section{S5: X-ray Crystallography}

\section{S5.1. X-ray of transporter 2 (CCDC 1999360)}

Single crystals of transporter $2\left(\mathrm{C}_{23} \mathrm{H}_{17} \mathrm{~N}_{5} \mathrm{OF}_{6} \mathrm{~S}\right)$ were obtained by slow evaporation of DMSO solution of 2. A suitable crystal was selected and in Paratone on a micromount on a SuperNova, Dual, $\mathrm{Cu}$ at home/near, Atlas diffractometer. The crystal was kept at $100 \mathrm{~K}$ during data collection. Using Olex $2^{[5]}$, the structure was solved with the ShelXS ${ }^{[6]}$ structure solution program using Direct Methods and refined with the ShelXL ${ }^{[7]}$ refinement package using Least Squares minimization.

Crystal Data for $\mathrm{C}_{23} \mathrm{H}_{17} \mathrm{~N}_{5} \mathrm{OF}_{6} \mathrm{~S}\left(M=525.47 \mathrm{~g} / \mathrm{mol}\right.$ ): orthorhombic, space group Pmc2 ${ }_{1}$ (no. 26), $a=6.841(2) \AA, b=17.047(3) \AA, c=19.783(4) \AA, V=2307.1(10) \AA^{3}, Z=4, T=100(2) \mathrm{K}$, $\mu(\mathrm{Cu} \mathrm{K \alpha})=1.943 \mathrm{~mm}^{-1}$, Dcalc $=1.513 \mathrm{~g} / \mathrm{cm}^{3}, 3102$ reflections measured $\left(8.94^{\circ} \leq 2 \Theta \leq 147.086^{\circ}\right)$, 3102 unique $\left(R_{\text {int }}=\right.$ ?, $\left.R_{\text {sigma }}=0.0551\right)$ which were used in all calculations. The final $R_{1}$ was 0.1265 $(\mathrm{I}>2 \sigma(\mathrm{I}))$ and $w R_{2}$ was 0.3804 (all data).

Figure S44: An Olex2 depiction of the complex 7 with the displacement ellipsoids shown at the $50 \%$ level.

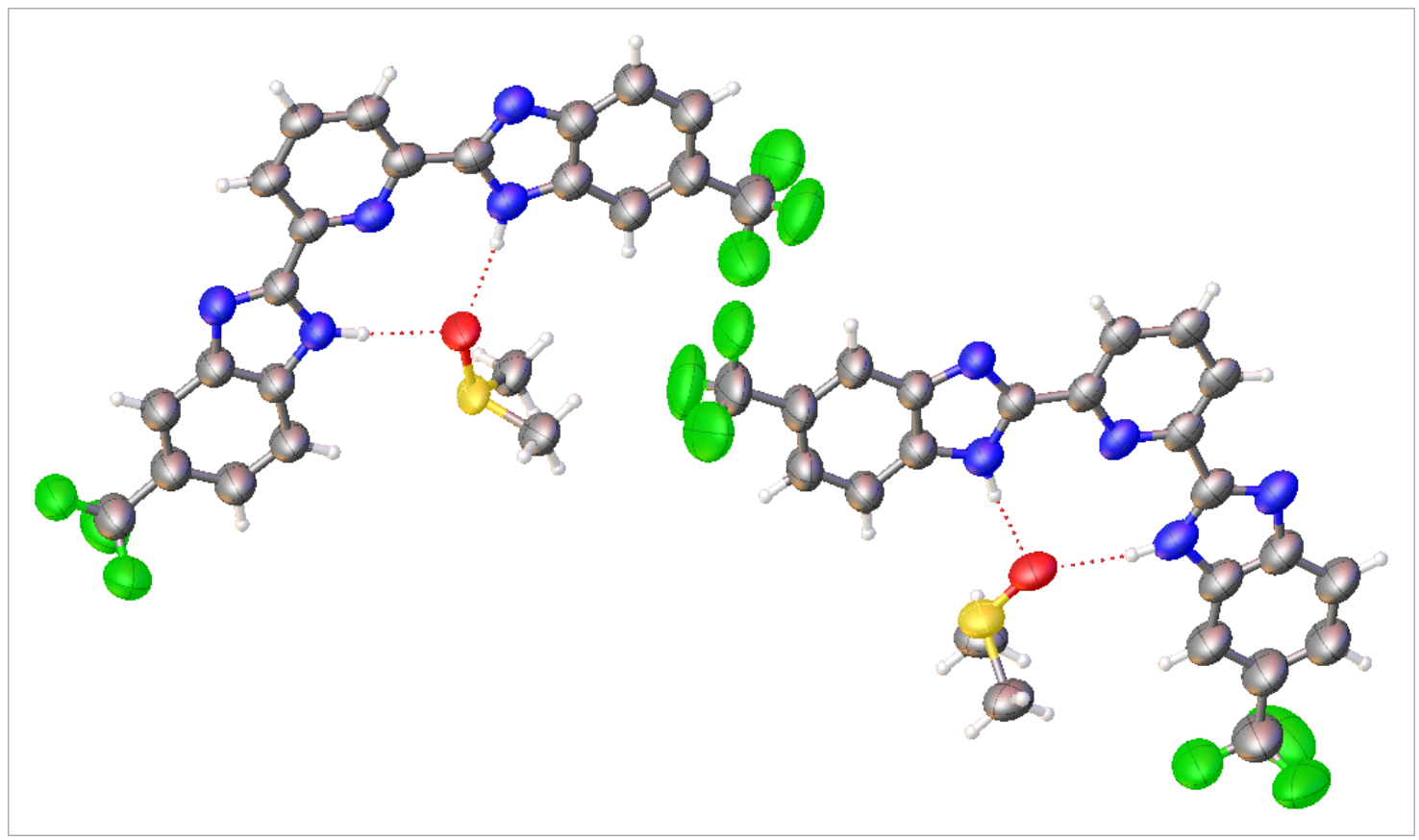




\section{S5.1. X-ray of complex 7 (CCDC 1999495)}

Single crystals of complex $7\left(\mathrm{C}_{26} \mathrm{H}_{13} \mathrm{AuClF}_{12} \mathrm{~N}_{5} \mathrm{O}\right)$ were obtained by slow evaporation of acetone solution of 7. A suitable crystal was selected and in Paratone on a micromount on a SuperNova, Dual, Cu at home/near, Atlas diffractometer. The crystal was kept at $100 \mathrm{~K}$ during data collection. Using Olex $2^{[5]}$, the structure was solved with the ShelXS ${ }^{[6]}$ structure solution program using Direct Methods and refined with the ShelXL ${ }^{[7]}$ refinement package using Least Squares minimization.

Crystal Data for $\mathrm{C}_{26} \mathrm{H}_{13} \mathrm{AuClF}_{12} \mathrm{~N}_{5} \mathrm{O}$ ( $\left.M=871.83 \mathrm{~g} / \mathrm{mol}\right)$ : orthorhombic, space group $\mathrm{P} 2{ }_{1}{ }_{2}{ }_{1}{ }_{1}$ (no. 19), $a=4.9760(2) \AA, b=18.4846(6) \AA, c=29.9692(9) \AA, V=2756.57(16) \AA^{3}, Z=4, T=$ $100(2) \mathrm{K}, \mu($ CuKa $)=11.990 \mathrm{~mm}^{-1}$, Dcalc $=2.101 \mathrm{~g} / \mathrm{cm}^{3}, 10634$ reflections measured $\left(9.57^{\circ} \leq 2 \Theta\right.$ $\left.\leq 146.43^{\circ}\right), 5220$ unique $\left(R_{\text {int }}=0.0665, R_{\text {sigma }}=0.0718\right)$ which were used in all calculations. The final $R_{1}$ was $0.0895(\mathrm{I}>2 \sigma(\mathrm{I}))$ and $w R_{2}$ was 0.2353 (all data).

Figure S45: An Olex2 depiction of the complex 7 with the displacement ellipsoids shown at the $50 \%$ level.

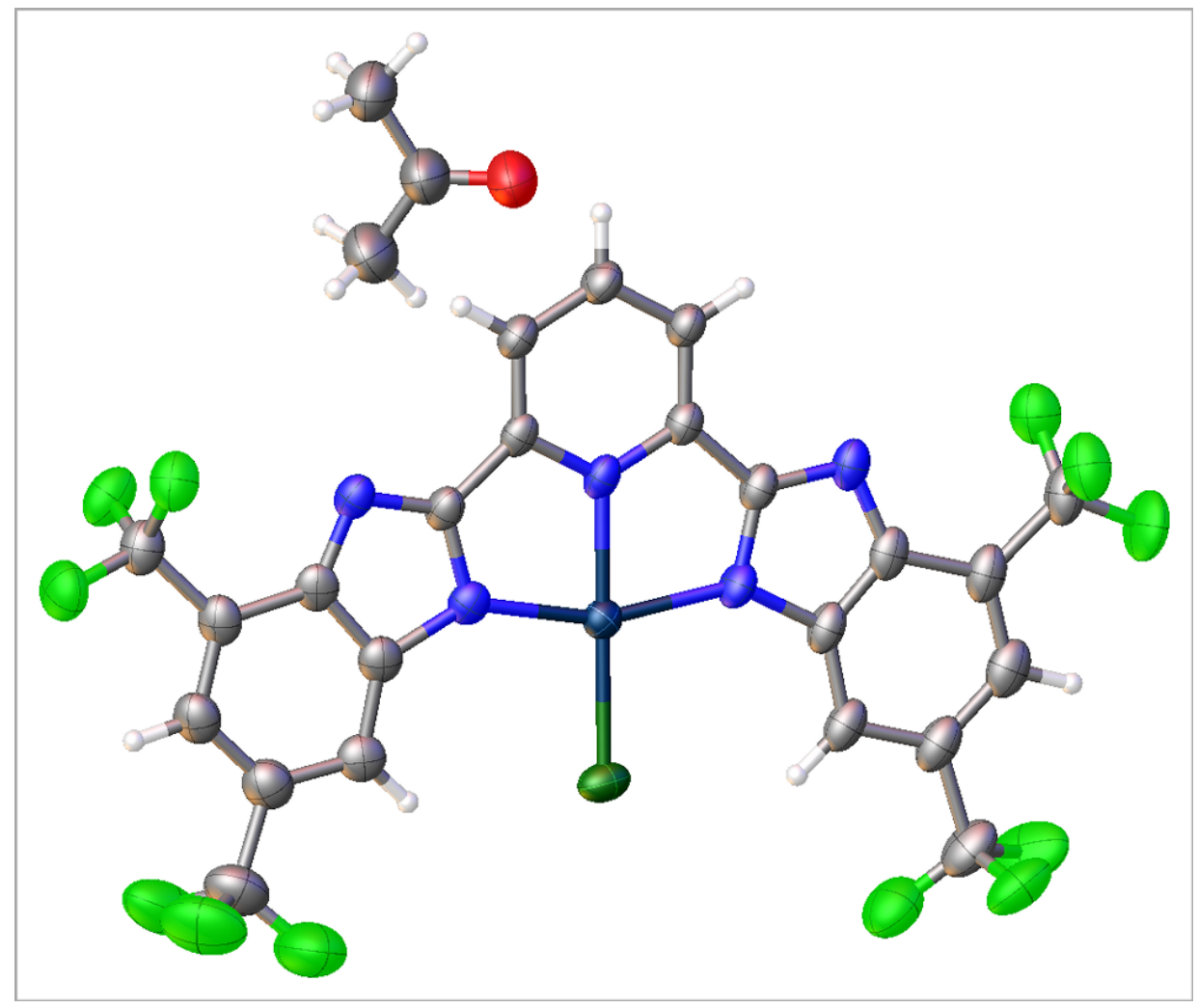




\section{S5.1. X-ray of transporter 8}

Single crystals of transporter $8\left(\mathrm{C}_{33} \mathrm{H}_{28} \mathrm{AuF}_{9} \mathrm{~N}_{8} \mathrm{O}_{3} \mathrm{~S}\right)$ were crystalized by a mixture of acetonitrile and diethyl ether solution of $\mathbf{8}$. A suitable crystal was selected and in Paratone on a micromount on a SuperNova, Dual, $\mathrm{Cu}$ at home/near, Atlas diffractometer. The crystal was kept at $100 \mathrm{~K}$ during data collection. Using Olex $2^{[5]}$, the structure was solved with the ShelXS ${ }^{[6]}$ structure solution program using Direct Methods and refined with the ShelXL ${ }^{[7]}$ refinement package using Least Squares minimization.

Crystal Data for $\mathrm{C}_{33} \mathrm{H}_{28} \mathrm{AuF}_{9} \mathrm{~N}_{8} \mathrm{O}_{3} \mathrm{~S}(\mathrm{M}=984.66 \mathrm{~g} / \mathrm{mol})$ : monoclinic, space group $\mathrm{P} 21 / \mathrm{n}$ (no. 14), $a=15.51220(20) \AA, b=16.2596(2) \AA, c=28.5690(3) \AA, \beta=95.2011(11)^{\circ}, V=7176.06(16) \AA^{3}$, $Z=8, T=100(2) \mathrm{K}, \mu(\mathrm{Cu} \mathrm{K \alpha})=9.101 \mathrm{~mm}^{-1}$, Dcalc $=1.823 \mathrm{~g} / \mathrm{cm}^{3}, 12657$ reflections measured $\left(7.894^{\circ} \leq 2 \Theta \leq 133.198^{\circ}\right), 12657$ unique $\left(R_{\text {int }}=\right.$ ?, $\left.R_{\text {sigma }}=0.0511\right)$ which were used in all calculations. The final $R_{1}$ was $0.0380(\mathrm{I}>2 \sigma(\mathrm{I}))$ and $w R_{2}$ was 0.0907 (all data).

Figure S46: An Olex2 depiction of the complex 7 with the displacement ellipsoids shown at the $50 \%$ level.

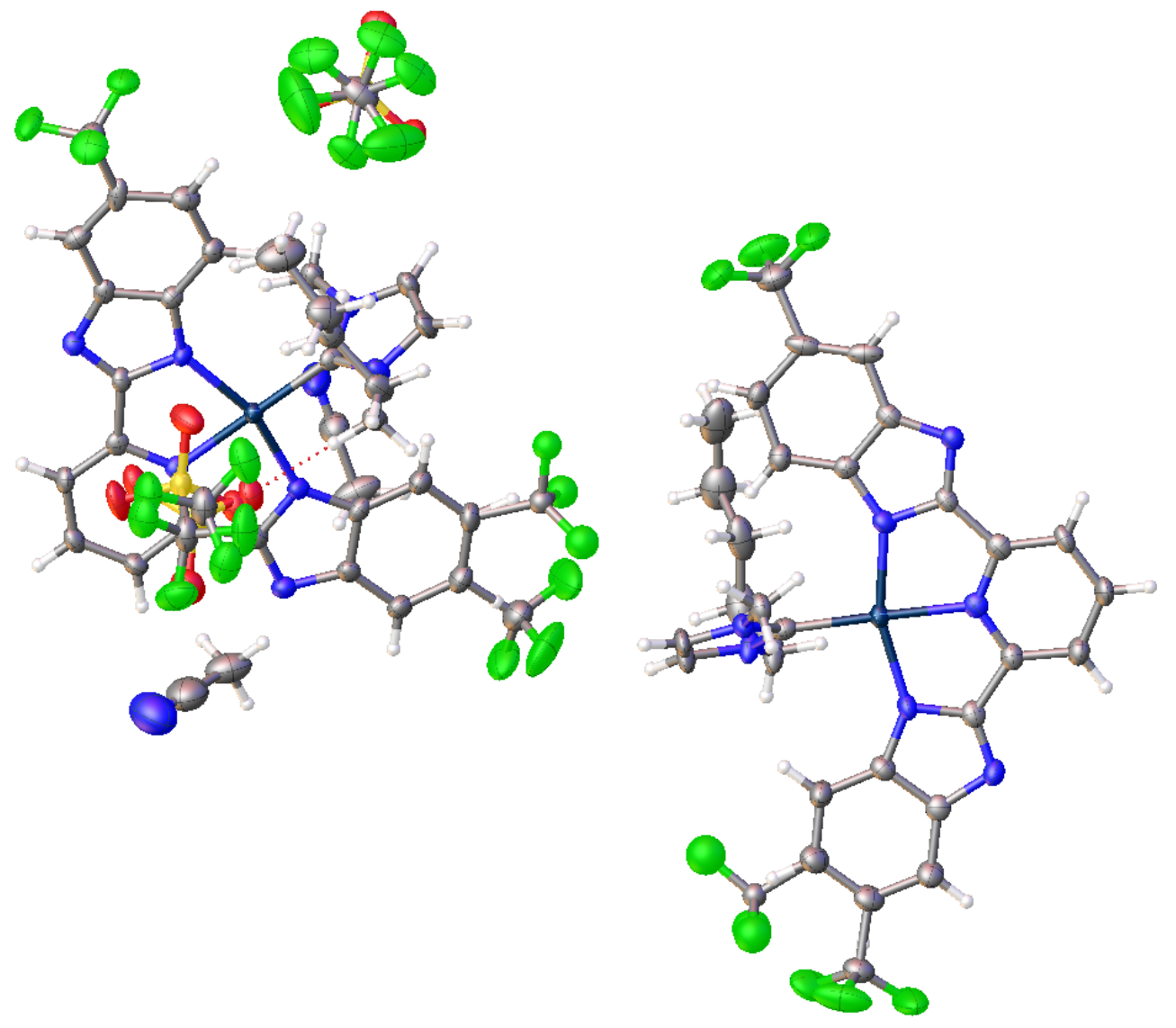




\section{S5.2. X-ray of complex 9}

Single crystals of complex 9 were obtained by slow vapour diffusion of $\mathrm{Et}_{2} \mathrm{O}$ into an acetone solution of 9. A suitable crystal was selected and in Paratone on a micromount on a SuperNova, Dual, Cu at home/near, Atlas diffractometer. The crystal was kept at $100 \mathrm{~K}$ during data collection. Using Olex $2^{[5]}$, the structure was solved with the ShelXS ${ }^{[6]}$ structure solution program using Direct Methods and refined with the ShelXL ${ }^{[7]}$ refinement package using Least Squares minimization.

Crystal Data for $\mathrm{C}_{36} \mathrm{H}_{29} \mathrm{~N}_{7} \mathrm{O}_{4} \mathrm{~F}_{15} \mathrm{SAu}(M=1137.69 \mathrm{~g} / \mathrm{mol}$ ): triclinic, space group P-1 (no. 2), $a=11.2877(3) \AA, b=14.0974(3) \AA, c=14.9642(4) \AA, \alpha=109.821(2)^{\circ}, \beta=107.118(2)^{\circ}, \quad y=$ 102.3303(19) $)^{\circ}, \quad V=2006.66(9) \AA^{3}, \quad Z=2, \quad T=100(2) \mathrm{K}, \mu(\mathrm{CuKa})=8.474 \mathrm{~mm}^{-1}, \quad$ Dcalc $=$ $1.883 \mathrm{~g} / \mathrm{cm}^{3}, 14400$ reflections measured $\left(6.848^{\circ} \leq 2 \Theta \leq 145.304^{\circ}\right), 7742$ unique $\left(R_{\text {int }}=0.0278\right.$, $\left.R_{\text {sigma }}=0.0382\right)$ which were used in all calculations. The final $R_{1}$ was $0.0337(\mathrm{I}>2 \sigma(\mathrm{I}))$ and $w R_{2}$ was 0.0859 (all data).

Figure S47: An Olex2 depiction of the complex 7 with the displacement ellipsoids shown at the $50 \%$ level.

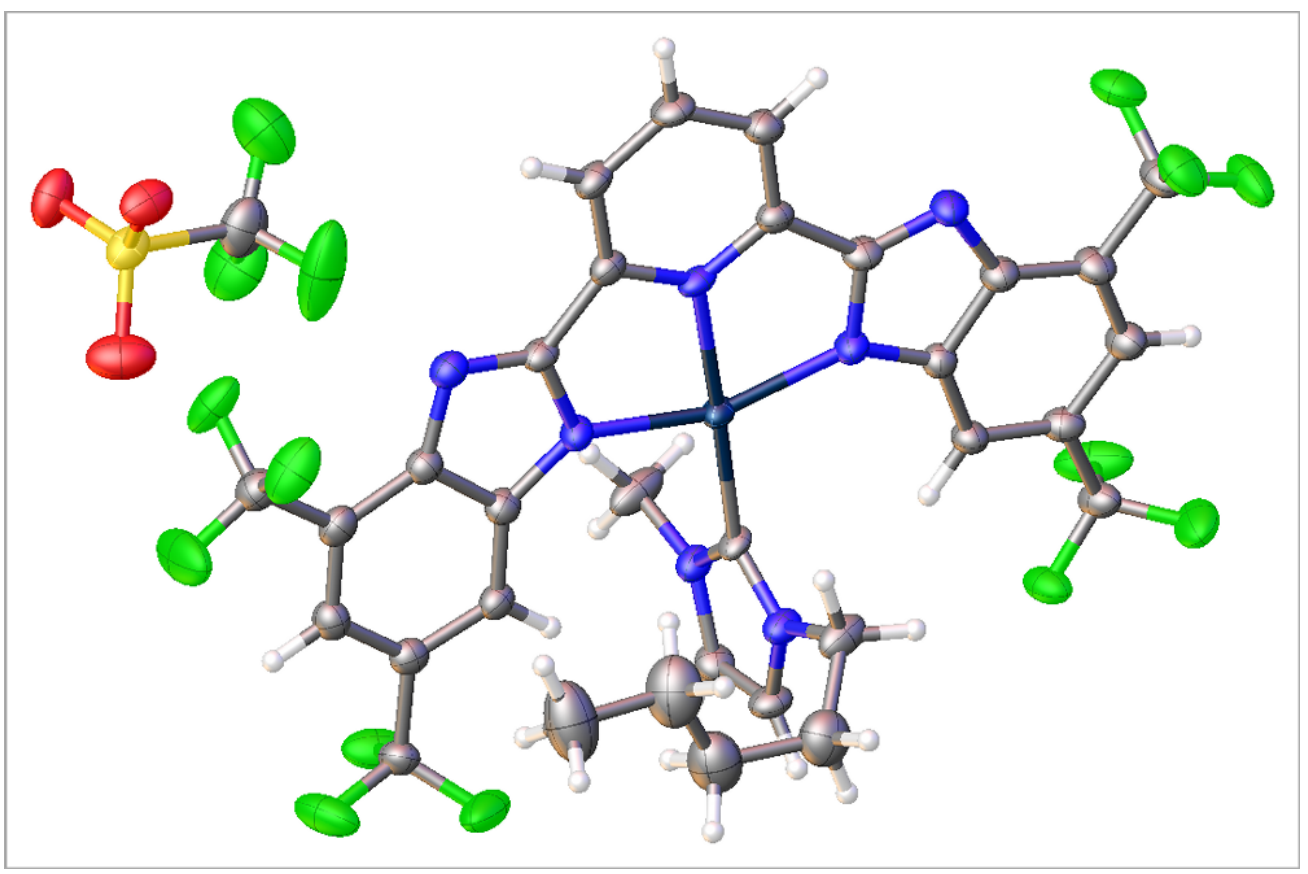




\section{S5.2. X-ray of 2.TEACI complex}

Single crystals of transporter 2 and chloride ion were obtained by slow vapour diffusion of $\mathrm{Et}_{2} \mathrm{O}$ into acetonitrile solution of $\mathbf{2}$ and 2 equivalents of TEACI. A suitable crystal was selected and in Paratone on a micromount on a SuperNova, Dual, $\mathrm{Cu}$ at home/near, Atlas diffractometer. The crystal was kept at $100 \mathrm{~K}$ during data collection. Using Olex $2^{[5]}$, the structure was solved with the ShelXS ${ }^{[6]}$ structure solution program using Direct Methods and refined with the ShelXL ${ }^{[7]}$ refinement package using Least Squares minimization.

Crystal Data for $\mathrm{C}_{50} \mathrm{H}_{48} \mathrm{ClF}_{12} \mathrm{~N}_{11} \mathrm{O}_{3}(M=1114.44 \mathrm{~g} / \mathrm{mol})$ : triclinic, space group P-1 (no. 2), $a=11.3690(16) \AA, b=11.800(2) \AA, c=19.141(3) \AA, \alpha=100.941(16)^{\circ}, \beta=90.546(14)^{\circ}, y=$ 104.405(14) $)^{\circ}, V=2437.5(7) \AA^{3}, Z=2, T=100(2) \mathrm{K}, \mu($ CuKa $)=1.605 \mathrm{~mm}^{-1}$, Dcalc $=1.518 \mathrm{~g} / \mathrm{cm}^{3}$, 19563 reflections measured $\left(7.892^{\circ} \leq 2 \Theta \leq 147.71^{\circ}\right), 9489$ unique $\left(R_{\text {int }}=0.1261, R_{\text {sigma }}=0.1672\right)$ which were used in all calculations. The final $R_{1}$ was $0.1404(\mathrm{I}>2 \sigma(\mathrm{I}))$ and $w R_{2}$ was 0.4052 (all data).

Figure S48: An Olex2 depiction of the 2. TEACl complex with the displacement ellipsoids shown at the $50 \%$ level.

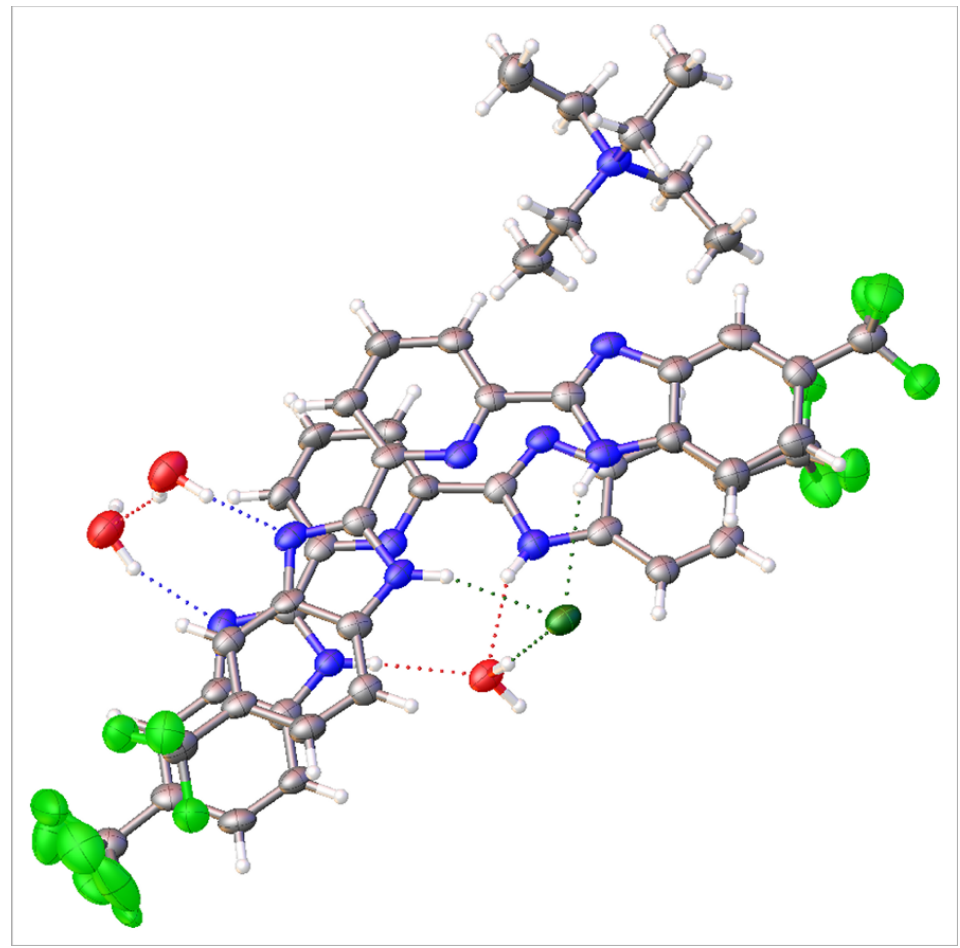




\section{S6: Anion Transport Studies}

\section{S6.1: General preparation for HPTS assays:}

Base-pulse 8-hydroxypyrene-1,3,6-trisulfonic acid trisodium salt (HPTS) assays were conducted using unilamellar 1-palmitoyl-2-oleoylphosphatidylcholine vesicles (POPC) with a mean diameter of $200 \mathrm{~nm}$ loaded with the $\mathrm{pH}$ sensitive fluorescence dye HPTS (1 $\mathrm{mM}) .{ }^{[8]}$ A chloroform solution of POPC $(\sim 30 \mathrm{mg} / \mathrm{mL})$ was evaoprated under vacuum and dried for at least $6 \mathrm{~h}$. The thin film was hydrated by the internal solution containing HPTS (1 $\mathrm{mM})$ and was subjected to nine freeze-thaw cycles followed by extrusion 25 times through a $200 \mathrm{~nm}$ polycarbonate membrane. Size exclusion chromatography using sephadex G-25 column and HPTS-free external solution as an eluent was conducted to remove unentrapped HPTS from the vesicles' solution. The internal and external solutions used were identical salt solution potassium gluconate (KGlu) or $\mathrm{KCl}$ (100 mM) buffered with $10 \mathrm{mM}$ HEPES at $\mathrm{pH}$ 7.0. Finally, for each measurement, external solution ( $\mathrm{KCl}$ or $\mathrm{KGlu}$ ) was used to dilute the lipid tock to obtain $2.5 \mathrm{~mL}$ lipid suspension containing $0.1 \mathrm{mM}$ lipid. A base pulse of $\mathrm{KOH}(25 \mu \mathrm{L}, 0.5 \mathrm{M})$ at a final concentration of $5 \mathrm{mM}$ was added to generate a transmembrane $\mathrm{pH}$ gradient. After the tested receptors were added, HPTS fluorescence ratio $\left(\lambda_{\mathrm{ex}}=460 \mathrm{~nm}, \lambda_{\mathrm{em}}=510 \mathrm{~nm}\right.$ divided by $\lambda_{\mathrm{ex}}=403 \mathrm{~nm}, \lambda_{\mathrm{em}}=510$ $\mathrm{nm}$ ) was recorded. Assisting ionophore (carbonyl cyanide phenylhydrazone (CCCP) or valinomycin) was used as a $5 \mu \mathrm{L}$ DMSO. Bovine serum albumins (BSA) was added to vesicles at $1 \mathrm{~mol} \%$ (with respect to lipid) and allowed to stir for 30 minutes to test if the transport is fatty acid independent, while, oleic acid ( $1 \mathrm{~mol} \%$ and $10 \mathrm{~mol} \%$ ) was used as a source of fatty acid to test if the transport is fatty acid dependent. Detergent $(25 \mu \mathrm{L})$ was added at 200 seconds to destroy the $\mathrm{pH}$ gradient to calibrate the assay.

Results are the average of at least three repeats and the fractional fluorescence intensity $\left(I_{f}\right)$ was determined using the following formula:

$$
I_{f}=\frac{R_{t}-R_{0}}{R_{d}-R_{0}}
$$


Where

- $R_{\mathrm{t}}$ is the fluorescence ratio at time $\mathrm{t}$.

- $R_{0}$ is the fluorescence ratio at time 0

- $R_{\mathrm{d}}$ is the fluorescence ratio after detergent addition.

Hill plots were determined for $\mathrm{KCl}$ and $\mathrm{KGlu}$ transport assays by conducting transport assays at different tested receptors concentrations. Receptor concentration vs fractional fluorescence intensity $I_{f}$ at $200 \mathrm{~s}$ (the endpoint of transport assay) were plotted and fitted to the Hill equation using Origin 2019b:

The following formula was used to calculate hill coefficients $(n)$ and $\mathrm{EC}_{50}(200 \mathrm{~s})$ values by fitting the curves to the following equation:

$$
y=y_{0}+\left(y_{\max }-y_{0}\right) \frac{x^{n}}{k^{n}+x^{n}}
$$

Where:

- $y$ is $I_{f}(200 \mathrm{~s})$ value of the ionophore at concentration $x$ (receptors concentration is expressed as ionophore to lipid molar ratio).

- $y_{0}$ is $l_{f}$ value at $200 \mathrm{~s}$, without addition of the ionophore.

- $y_{\max }$ is the maximum If value.

- $\quad n$ is the Hill coefficient, and $K$ is the $\mathrm{EC}_{50}(200 \mathrm{~s})$ value. 

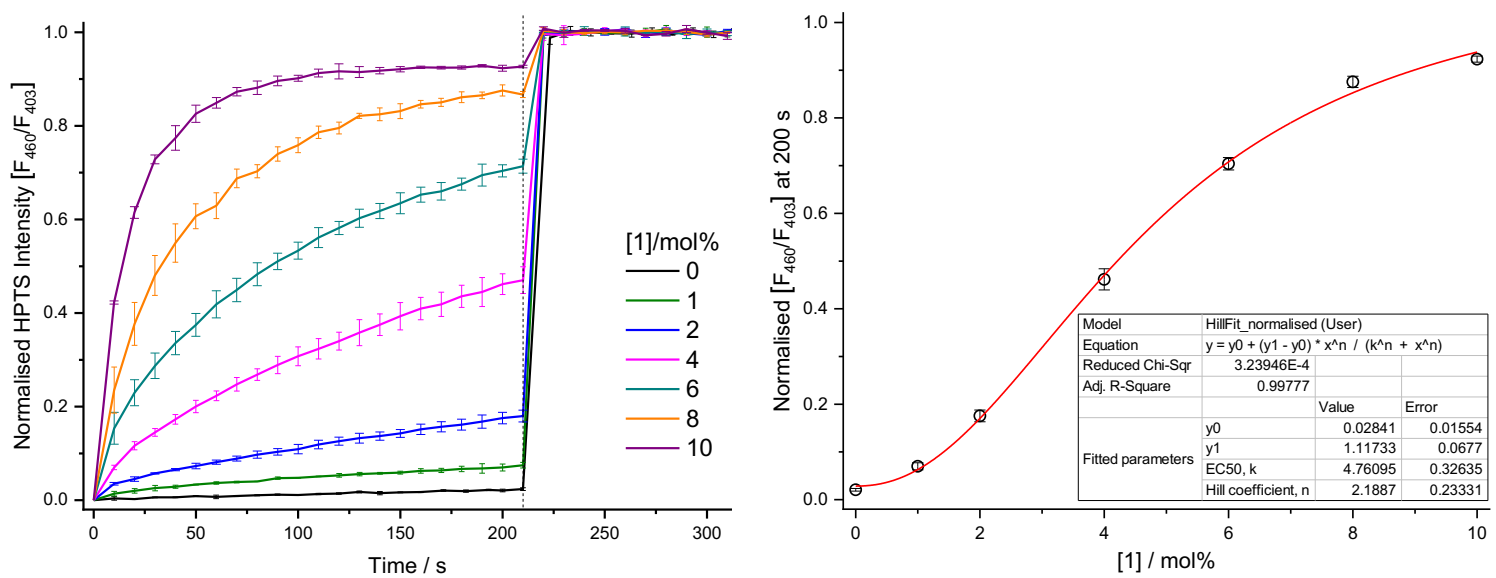

Figure S49: Hill plot analysis of $\mathrm{H}^{+} / \mathrm{Cl}^{-}$symport or $\mathrm{Cl}^{-} / \mathrm{OH}^{-}$antiport facilitated by compound 1 using $\mathrm{KCl}-\mathrm{KOH}$ assay from POPC vesicles loaded with $\mathrm{KCl}(100 \mathrm{mM})$, buffered to $\mathrm{pH} 7.0$ with HEPES $(10 \mathrm{mM})$. The test compound was added at 0 $s$ and detergent was added at $200 \mathrm{~s}$. Ionophore concentrations are shown as ionophore to lipid molar ratios. Error bars represent SD from at least three repeats.
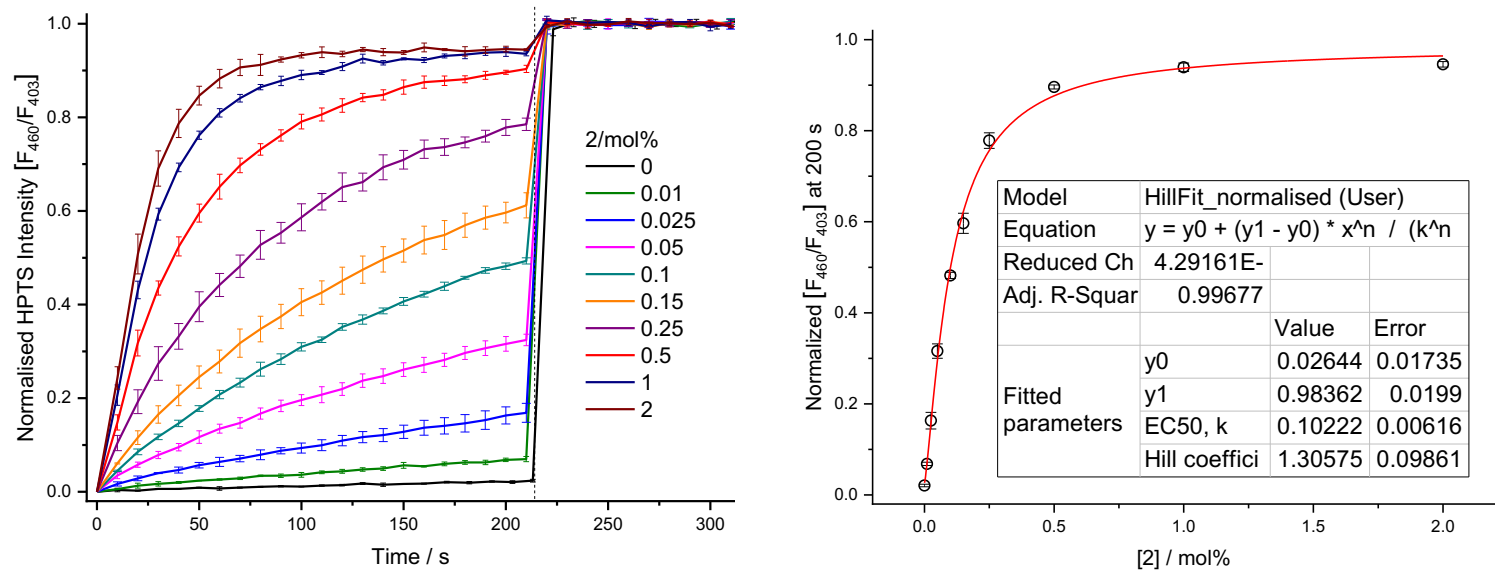

Figure S50: Hill plot analysis of $\mathrm{H}^{+} / \mathrm{Cl}^{-}$symport or $\mathrm{Cl}^{-} / \mathrm{OH}^{-}$antiport facilitated by compound 2 using $\mathrm{KCl}-\mathrm{KOH}$ assay from POPC vesicles loaded with $\mathrm{KCl}(100 \mathrm{mM})$, buffered to $\mathrm{pH} 7.0$ with HEPES $(10 \mathrm{mM})$. The test compound was added at $0 \mathrm{~s}$ and detergent was added at $200 \mathrm{~s}$. Ionophore concentrations are shown as ionophore to lipid molar ratios. Error bars represent SD from at least three repeats. 

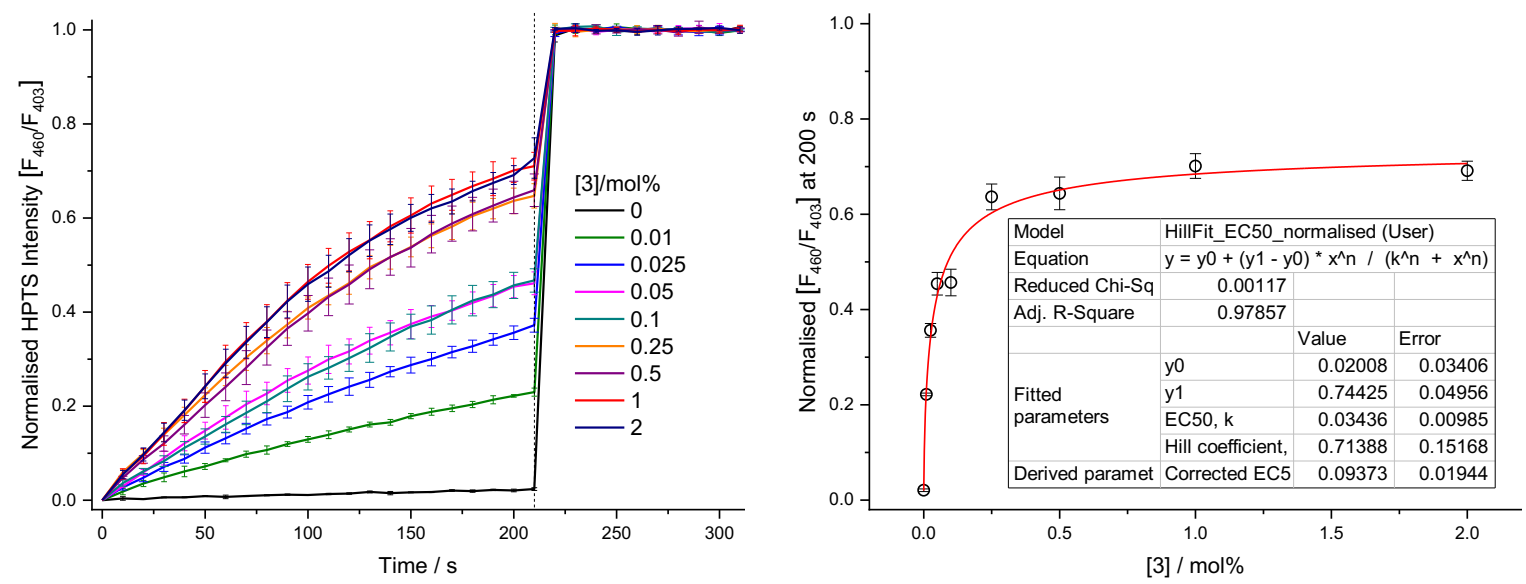

Figure S51: Hill plot analysis of $\mathrm{H}^{+} / \mathrm{Cl}^{-}$symport or $\mathrm{Cl} / \mathrm{OH}^{-}$antiport facilitated by compound 3 using $\mathrm{KCl}-\mathrm{KOH}$ assay from POPC vesicles loaded with $\mathrm{KCl}(100 \mathrm{mM})$, buffered to $\mathrm{pH} 7.0$ with HEPES $(10 \mathrm{mM})$. The test compound was added at $0 \mathrm{~s}$ and detergent was added at $200 \mathrm{~s}$. lonophore concentrations are shown as ionophore to lipid molar ratios. Error bars represent SD from at least three repeats.
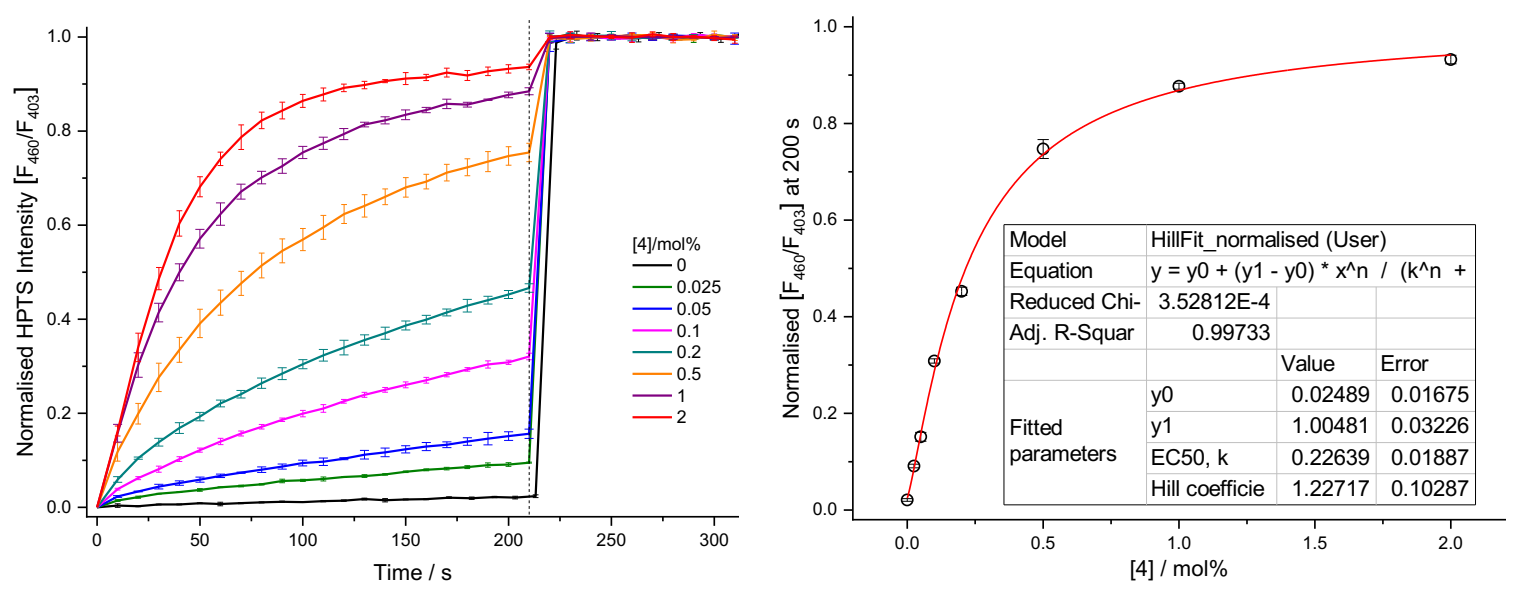

Figure S52: : Hill plot analysis of $\mathrm{H}^{+} / \mathrm{Cl}^{-}$symport or $\mathrm{Cl}^{-} / \mathrm{OH}^{-}$antiport facilitated by compound 4 using $\mathrm{KCl}-\mathrm{KOH}$ assay from POPC vesicles loaded with $\mathrm{KCl}(100 \mathrm{mM})$, buffered to $\mathrm{pH} 7.0$ with HEPES $(10 \mathrm{mM})$. The test compound was added at $0 \mathrm{~s}$ and detergent was added at $200 \mathrm{~s}$. lonophore concentrations are shown as ionophore to lipid molar ratios. Error bars represent SD from at least three repeats. 

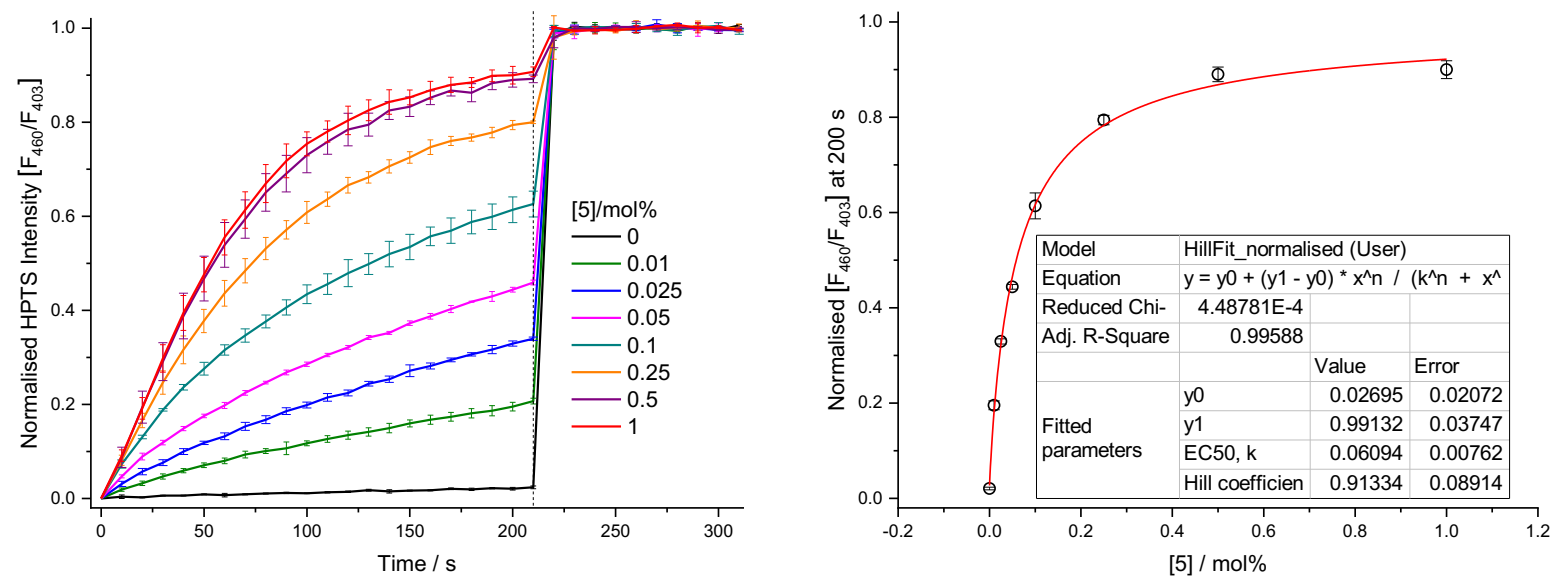

Figure S53: Hill plot analysis of $\mathrm{H}^{+} / \mathrm{Cl}^{-}$symport or $\mathrm{Cl} / \mathrm{OH}^{-}$antiport facilitated by compound 5 using $\mathrm{KCl}-\mathrm{KOH}$ assay from POPC vesicles loaded with $\mathrm{KCl}(100 \mathrm{mM})$, buffered to $\mathrm{pH} 7.0$ with HEPES $(10 \mathrm{mM})$. The test compound was added at $0 \mathrm{~s}$ and detergent was added at $200 \mathrm{~s}$. Ionophore concentrations are shown as ionophore to lipid molar ratios. Error bars represent SD from at least three repeats.

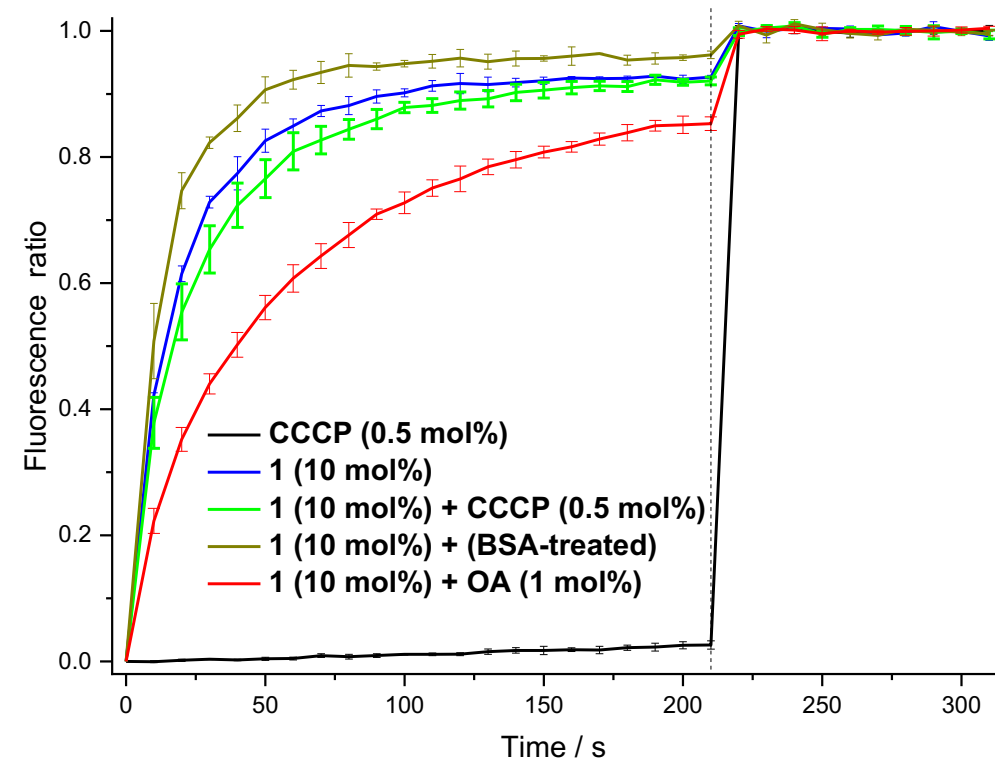

Figure S54: Using $\mathrm{KCl}-\mathrm{KOH}$ assay from POPC vesicles loaded with $\mathrm{KCl}(100 \mathrm{mM})$, buffered to $\mathrm{pH} 7.0$ with HEPES $(10 \mathrm{mM})$, different conditions were applied to determine the effect of addition of the protonphore cccp at $0.5 \mathrm{~mol} \%$ (to measure of chloride uniport solely), oleic acid at $1 \mathrm{~mol} \%$ (as a source of fatty acid) and BSA-treated lipid (to test if the transport is fatty acid dependent) on the rate of chloride transport of receptor 1 (10 mol\%). 


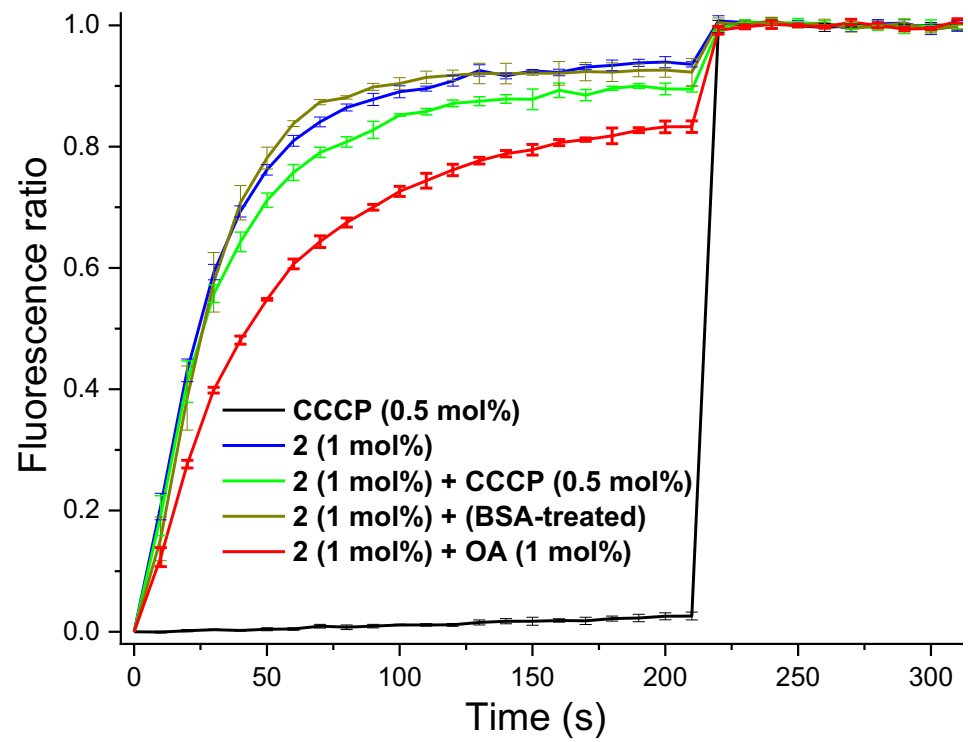

Figure S55: Using $\mathrm{KCl}-\mathrm{KOH}$ assay from POPC vesicles loaded with $\mathrm{KCl}(100 \mathrm{mM})$, buffered to $\mathrm{pH} 7.0$ with HEPES $(10 \mathrm{mM})$, different conditions were applied to determine the effect of addition of the protonphore cccp at $0.5 \mathrm{~mol} \%$ (as a measure of chloride uniport), oleic acid at $1 \mathrm{~mol} \%$ (as a source of fatty acid) and BSA-treated lipid (to test if the transport is fatty acid dependent) on the rate of chloride transport of receptor 2 ( $1 \mathrm{~mol} \%)$.

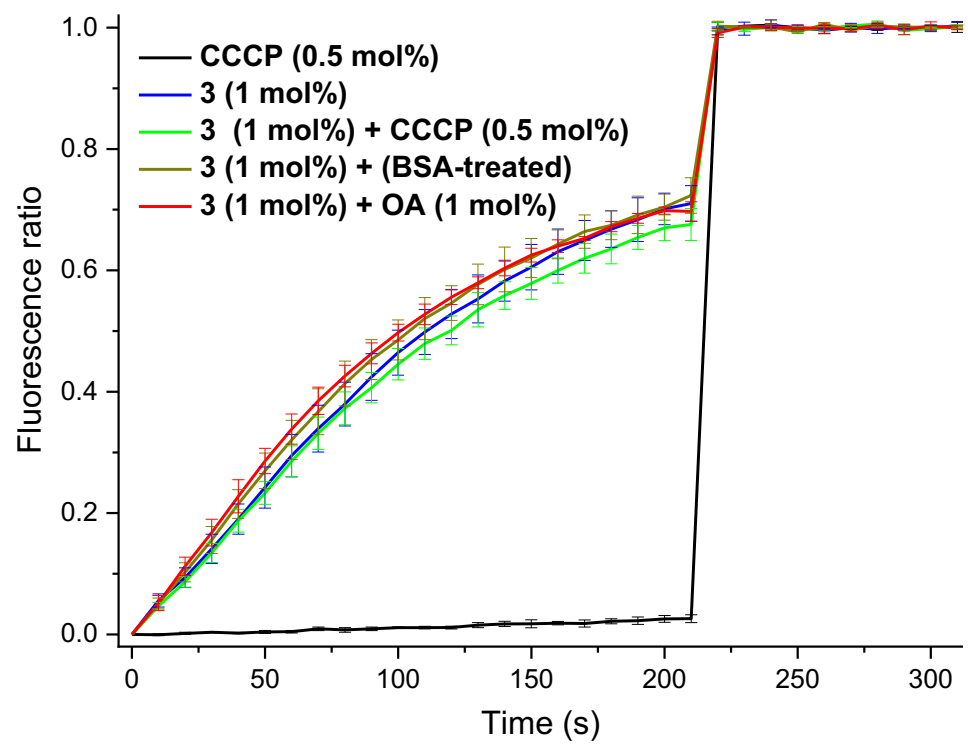

Figure S56: Using $\mathrm{KCl}-\mathrm{KOH}$ assay from POPC vesicles loaded with $\mathrm{KCl}(100 \mathrm{mM})$, buffered to $\mathrm{pH} 7.0$ with HEPES $(10 \mathrm{mM})$, different conditions were applied to determine the effect of addition of the protonphore cccp at $0.5 \mathrm{~mol} \%$ (as a measure of chloride uniport), oleic acid at $1 \mathrm{~mol} \%$ (as a source of fatty acid) and BSA-treated lipid (to test if the transport is fatty acid dependent) on the rate of chloride transport of receptor $3(1 \mathrm{~mol} \%)$. 


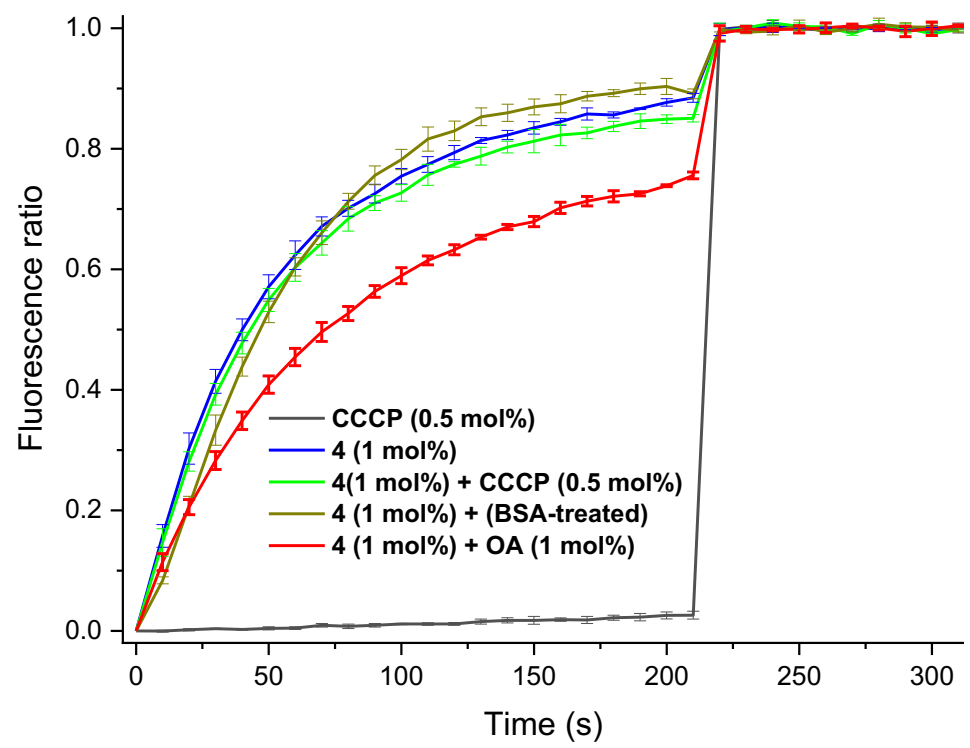

Figure S57: Using $\mathrm{KCl}-\mathrm{KOH}$ assay from POPC vesicles loaded with $\mathrm{KCl}(100 \mathrm{mM})$, buffered to $\mathrm{pH} 7.0$ with HEPES $(10 \mathrm{mM})$, different conditions were applied to determine the effect of addition of the protonphore cccp at $0.5 \mathrm{~mol} \%$ (as a measure of chloride uniport), oleic acid at $1 \mathrm{~mol} \%$ (as a source of fatty acid) and BSA-treated lipid (to test if the transport is fatty acid dependent) on the rate of chloride transport of receptor 4 (1 mol\%).

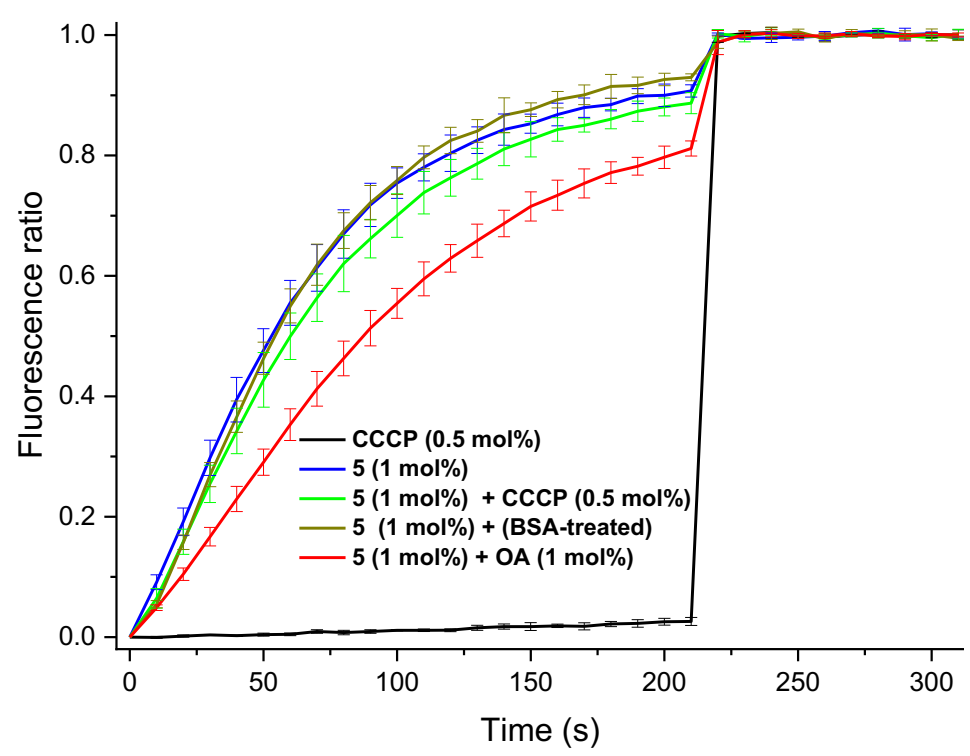

Figure S58: Using $\mathrm{KCl}-\mathrm{KOH}$ assay from POPC vesicles loaded with $\mathrm{KCl}(100 \mathrm{mM})$, buffered to $\mathrm{pH} 7.0$ with HEPES $(10 \mathrm{mM})$, different conditions were applied to determine the effect of addition of the protonphore cccp at $0.5 \mathrm{~mol} \%$ (as a measure of chloride uniport), oleic acid at $1 \mathrm{~mol} \%$ (as a source of fatty acid) and BSA-treated lipid (to test if the transport is fatty acid dependent) on the rate of chloride transport of receptor 5 ( $1 \mathrm{~mol} \%)$. 

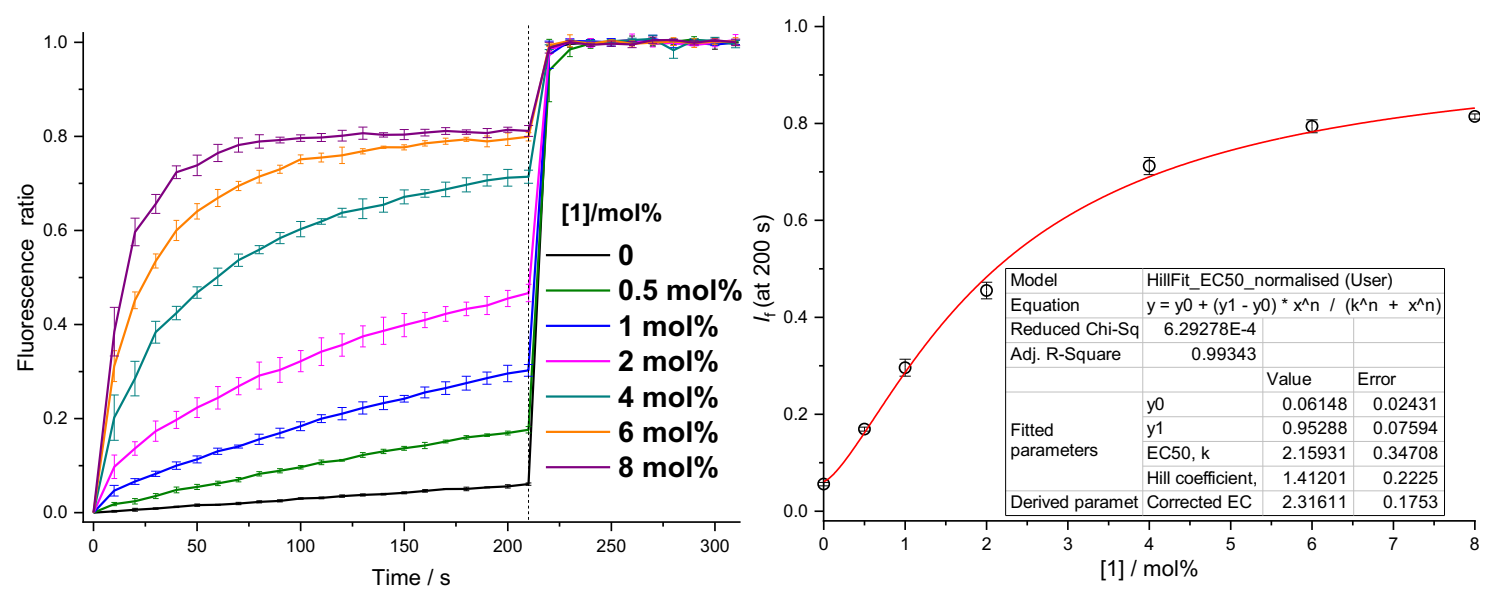

Figure S59: Hill plot analysis of $\mathrm{H}^{+}$flux facilitated by compound 1 using $\mathrm{KGlu}-\mathrm{KOH}$ assay from POPC vesicles loaded with KGlu (100 mM), buffered to pH 7.0 with HEPES (10 mM). The test compound was added at $0 \mathrm{~s}$ and detergent was added at $200 \mathrm{~s}$. Ionophore concentrations are shown as ionophore to lipid molar ratios. Error bars represent SD from at least three repeats.
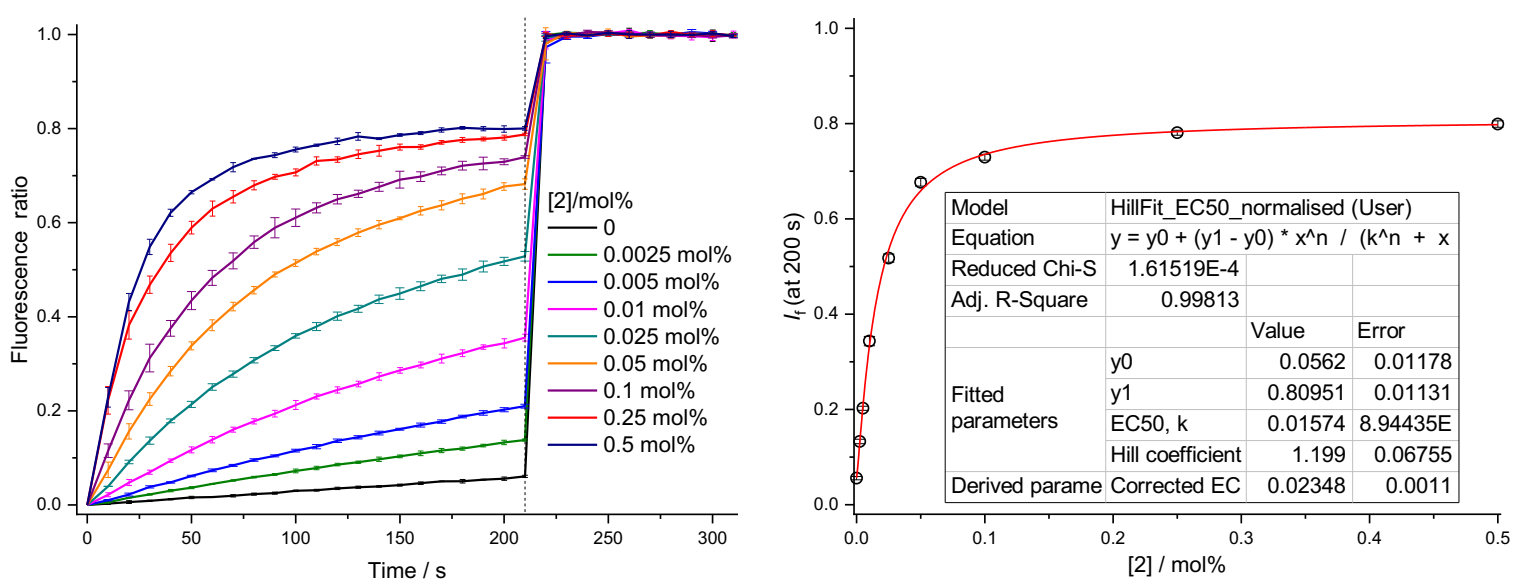

Figure S60: Hill plot analysis of $\mathrm{H}^{+}$flux facilitated by compound 2 using $\mathrm{KGlu}-\mathrm{KOH}$ assay from POPC vesicles loaded with KGlu (100 mM), buffered to $\mathrm{pH} 7.0$ with HEPES $(10 \mathrm{mM})$. The test compound was added at $0 \mathrm{~s}$ and detergent was added at $200 \mathrm{~s}$. Ionophore concentrations are shown as ionophore to lipid molar ratios. Error bars represent SD from at least three repeats. 

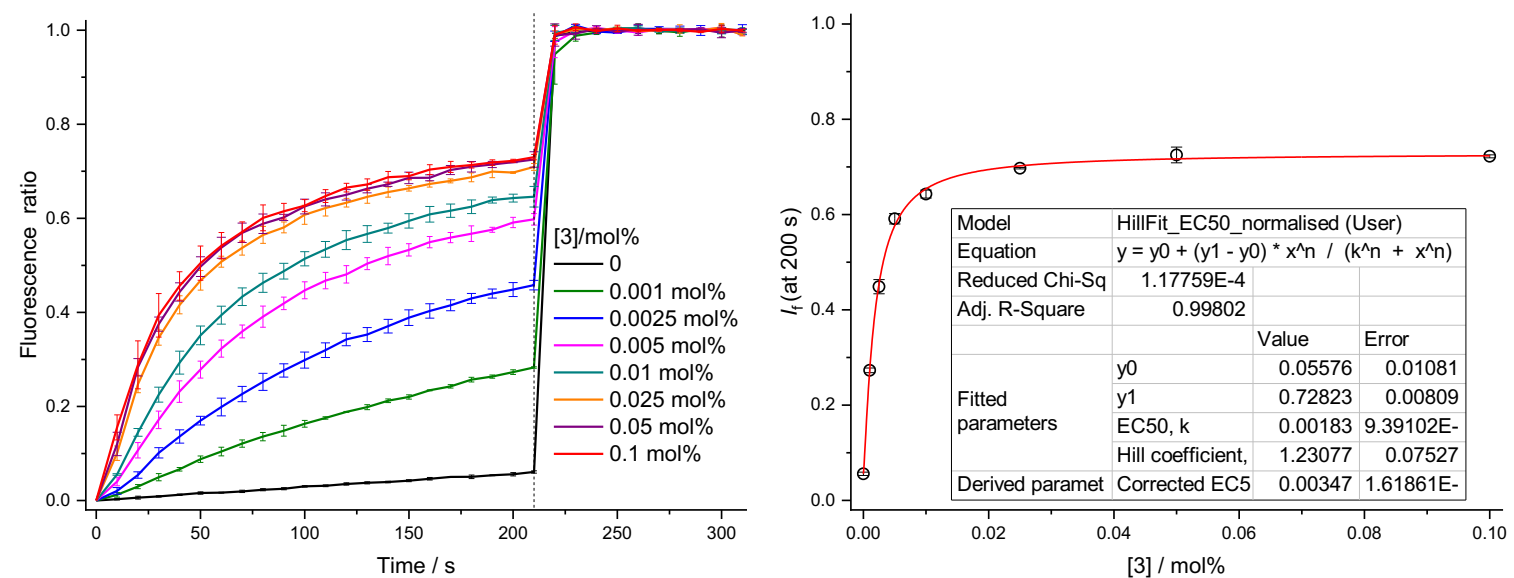

Figure S61: Hill plot analysis of $\mathrm{H}^{+}$flux facilitated by compound 3 using $\mathrm{KGlu}-\mathrm{KOH}$ assay from POPC vesicles loaded with KGlu $(100 \mathrm{mM})$, buffered to $\mathrm{pH} 7.0$ with HEPES $(10 \mathrm{mM})$. The test compound was added at $0 \mathrm{~s}$ and detergent was added at $200 \mathrm{~s}$. Ionophore concentrations are shown as ionophore to lipid molar ratios. Error bars represent SD from at least three repeats.
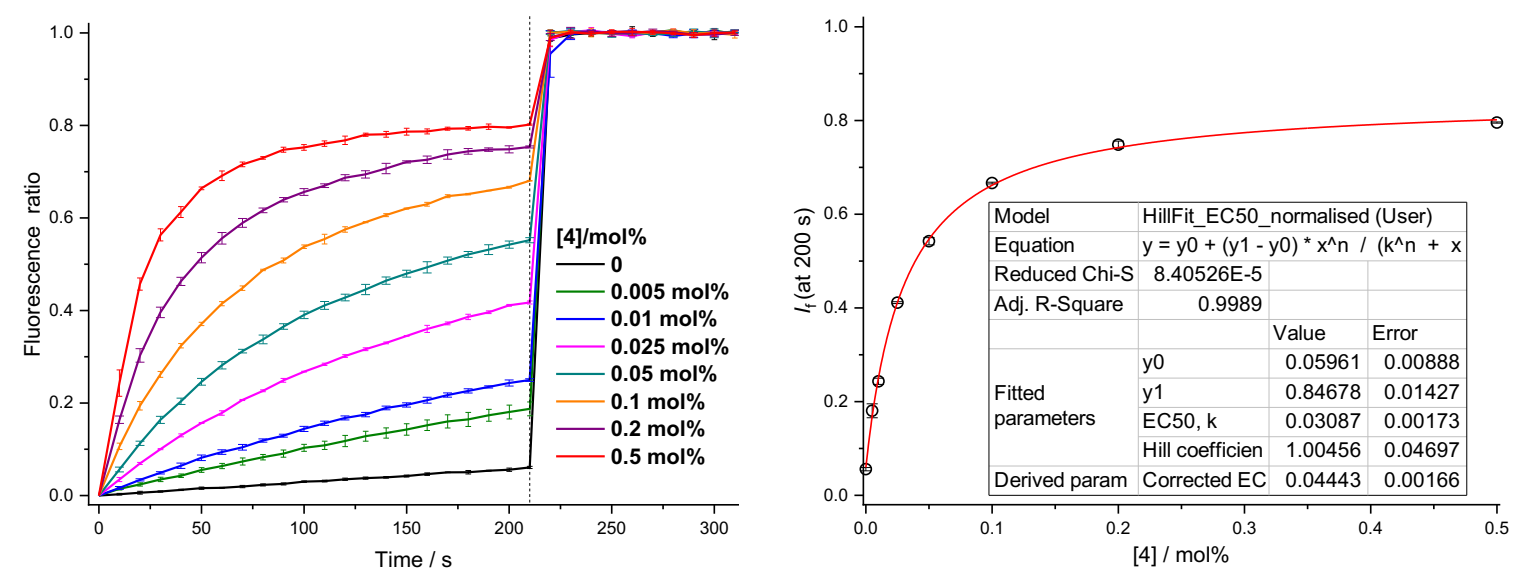

Figure S62: Hill plot analysis of $\mathrm{H}^{+}$flux facilitated by compound $\mathbf{4}$ using $\mathrm{KGlu}-\mathrm{KOH}$ assay from POPC vesicles loaded with KGlu $(100 \mathrm{mM})$, buffered to $\mathrm{pH} 7.0$ with HEPES $(10 \mathrm{mM})$. The test compound was added at $0 \mathrm{~s}$ and detergent was added at $200 \mathrm{~s}$. Ionophore concentrations are shown as ionophore to lipid molar ratios. Error bars represent SD from at least three repeats. 

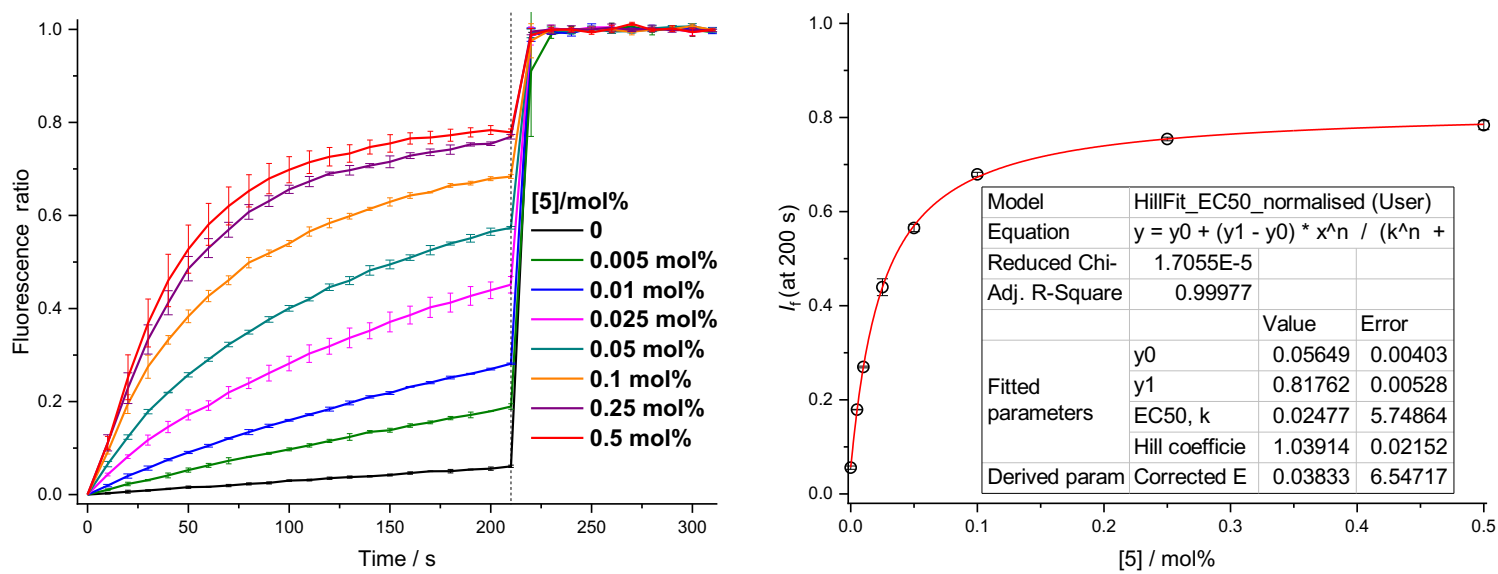

Figure S63: Hill plot analysis of $\mathrm{H}^{+}$flux facilitated by compound $\mathbf{5}$ using $\mathrm{KGlu}-\mathrm{KOH}$ assay from POPC vesicles loaded with KGlu $(100 \mathrm{mM})$, buffered to $\mathrm{pH} 7.0$ with HEPES (10 mM). The test compound was added at $0 \mathrm{~s}$ and detergent was added at $200 \mathrm{~s}$. lonophore concentrations are shown as ionophore to lipid molar ratios. Error bars represent SD from at least three repeats.

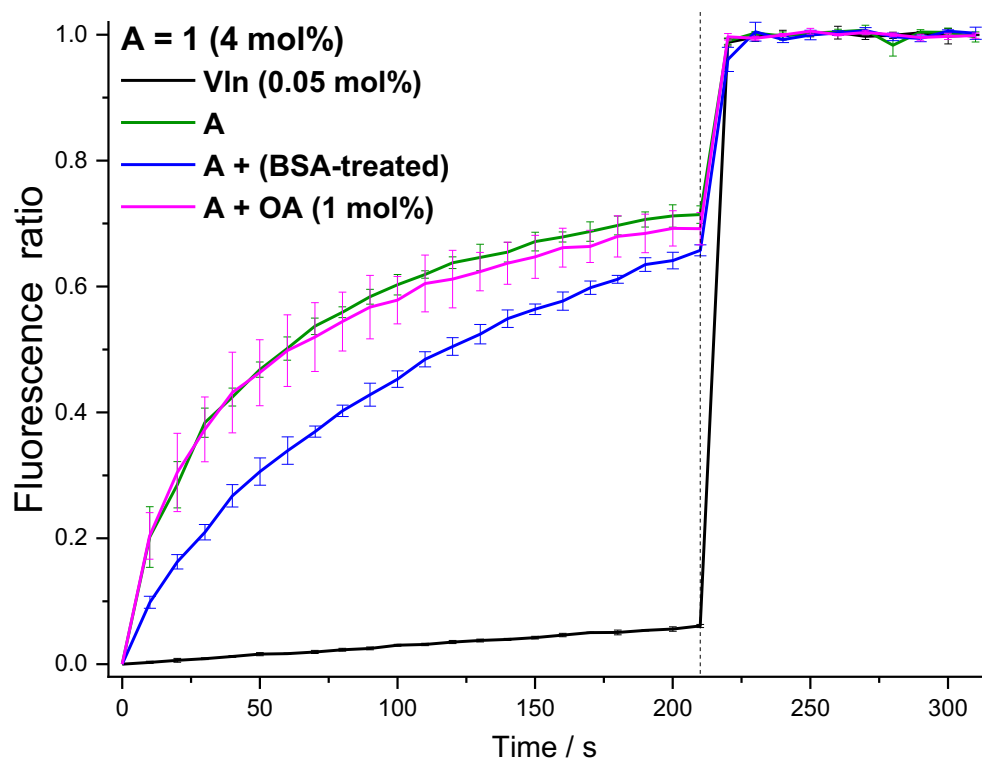

Figure S64: Using KGlu-KOH assay from POPC vesicles loaded with KGlu (100 mM), buffered to $\mathrm{pH} 7.0$ with HEPES $(10 \mathrm{mM})$, different conditions were applied to determine the effect of addition of the ionophore valinomycin at $0.05 \mathrm{~mol} \%$ (as a measure of $\mathrm{H}^{+}$flux), oleic acid at $1 \mathrm{~mol} \%$ (as a source of fatty acid) and BSA-treated lipid (to test if the transport is fatty acid dependent) on the rate of chloride transport of receptor 1 (4 mol\%). 


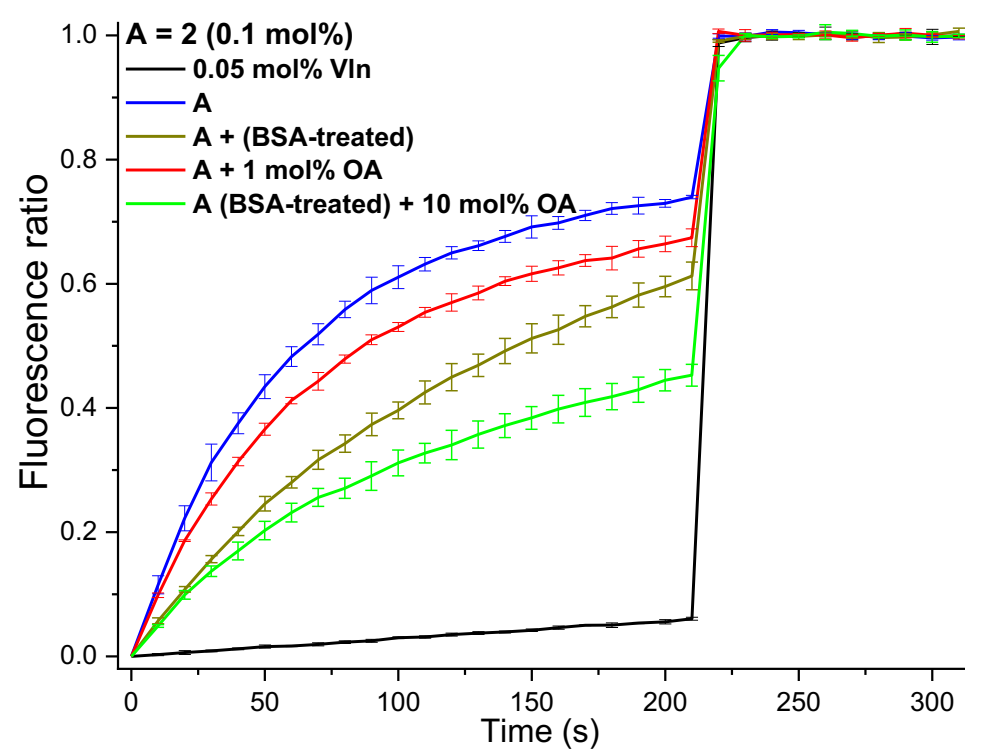

Figure S65: Using KGlu-KOH assay from POPC vesicles loaded with KGlu (100 mM), buffered to $\mathrm{pH} 7.0$ with HEPES $(10 \mathrm{mM})$, different conditions were applied to determine the effect of addition of the ionophore valinomycin at $0.05 \mathrm{~mol} \%$ (as a measure of $\mathrm{H}^{+}$flux), oleic acid at 1 mol\%AND $10 \mathrm{~mol} \%$ (as a source of fatty acid) and BSA-treated lipid (to test if the transport is fatty acid dependent) on the rate of chloride transport of receptor $2(0.1 \mathrm{~mol} \%)$.

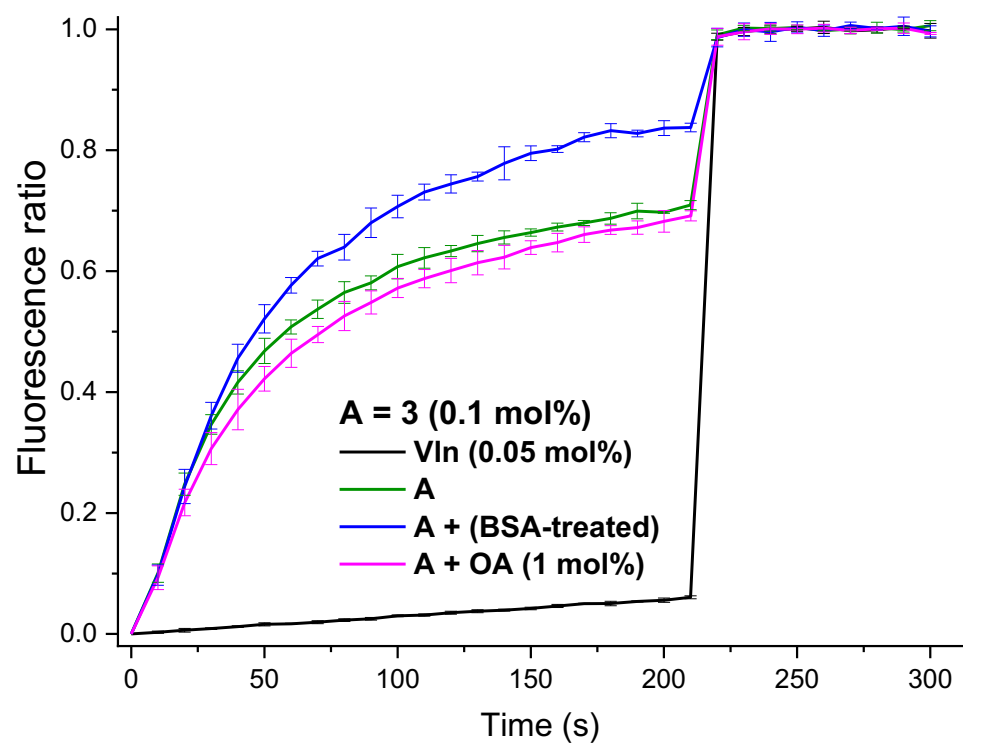

FigureS66: Using KGlu-KOH assay from POPC vesicles loaded with KGlu (100 mM), buffered to $\mathrm{pH} 7.0$ with HEPES $(10 \mathrm{mM})$, different conditions were applied to determine the effect of addition of the ionophore valinomycin at $0.05 \mathrm{~mol} \%$ (as a measure of $\mathrm{H}^{+}$flux), oleic acid at $1 \mathrm{~mol} \%$ (as a source of fatty acid) and BSA-treated lipid (to test if the transport is fatty acid dependent) on the rate of chloride transport of receptor $3(0.1 \mathrm{~mol} \%)$. 


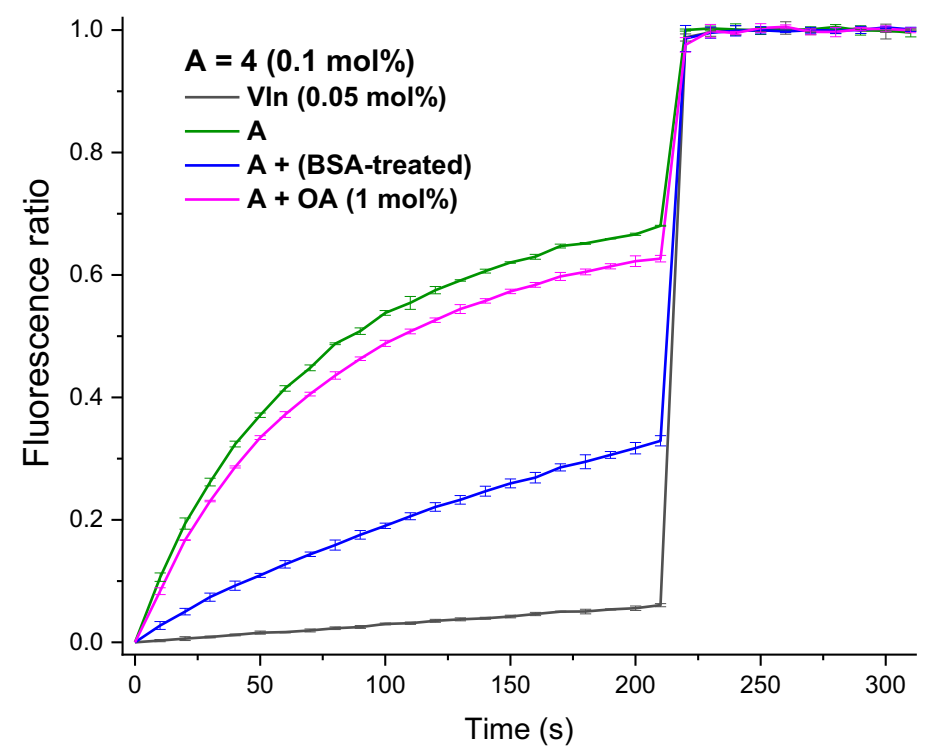

Figure S67: Using KGlu-KOH assay from POPC vesicles loaded with KGlu (100 mM), buffered to $\mathrm{pH} 7.0$ with HEPES $(10 \mathrm{mM})$, different conditions were applied to determine the effect of addition of the ionophore valinomycin at $0.05 \mathrm{~mol} \%$ (as a measure of $\mathrm{H}^{+}$flux), oleic acid at $1 \mathrm{~mol} \%$ (as a source of fatty acid) and BSA-treated lipid (to test if the transport is fatty acid dependent) on the rate of chloride transport of receptor $4(0.1 \mathrm{~mol} \%)$.

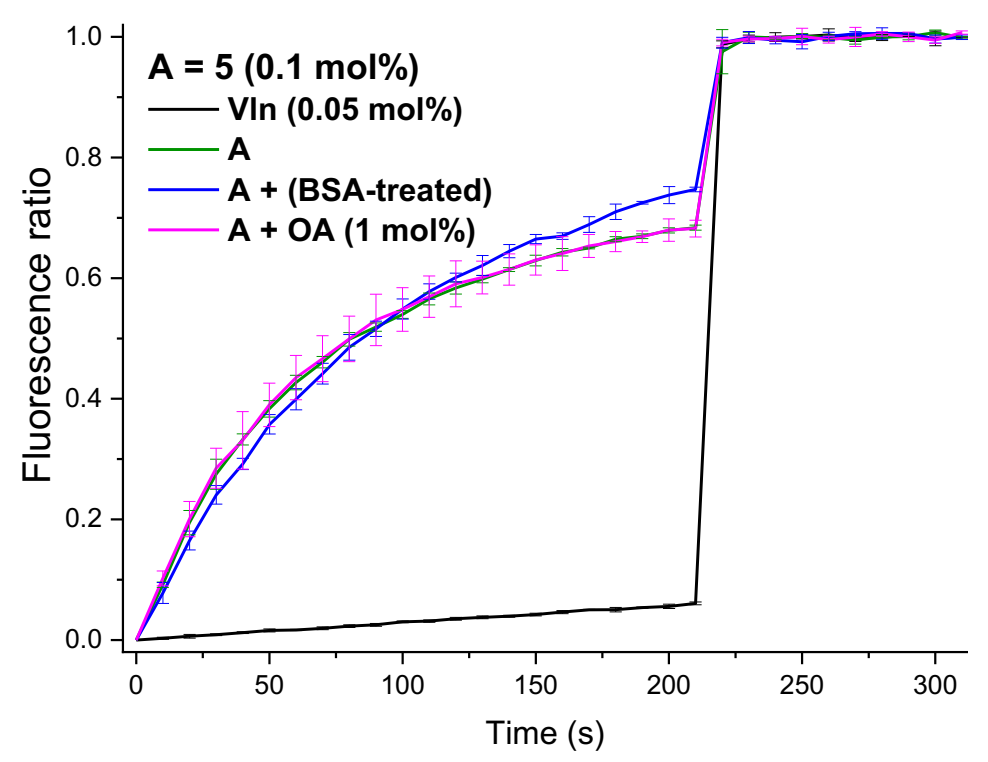

Figure S68: Using KGlu-KOH assay from POPC vesicles loaded with KGlu (100 mM), buffered to $\mathrm{pH} 7.0$ with HEPES $(10 \mathrm{mM})$, different conditions were applied to determine the effect of addition of the ionophore valinomycin at $0.05 \mathrm{~mol} \%$ (as a measure of $\mathrm{H}^{+}$flux), oleic acid at $1 \mathrm{~mol} \%$ (as a source of fatty acid) and BSA-treated lipid (to test if the transport is fatty acid dependent) on the rate of chloride transport of receptor $5(0.1 \mathrm{~mol} \%)$. 


\section{S6.2: Chloride vs anions selectivity assay:}

Unilamellar vesicles were prepared in a similar pattern to $\mathrm{KCl}-\mathrm{KOH}$ assay reported in section 6.1. Internal solution of each experiment contained $\mathrm{KCl}(100 \mathrm{mM})$ and HPTS (1 $\mathrm{mM}$ ) while five external solutions were used at $100 \mathrm{mM}$ with different salts $\mathrm{KCl}, \mathrm{KBr}, \mathrm{KI}$, $\mathrm{KNO}_{3}$ and $\mathrm{KClO}_{4}$. Both external and internal solutions are buffered with HEPES (10 mM) to $\mathrm{pH}$ 7.0. HPTS is a $\mathrm{pH}$ responsive probe that has two wavelengths corresponding to the acidic and basic forms, $403 \mathrm{~nm}$ and 460, respectively. Fluorescence ratio between acidic and basic forms was measured for the five receptors against chloride/anion ( $\mathrm{X}^{-}$) selectivity. If the anion $X^{-}$is being transported into the vesicles, it would transport as $\mathrm{HX}$ and so lowered the $\mathrm{pH}$ inside the vesicles, resulting in a negative Normalized HPTS value. However, if the chloride is being transported out along with the proton gradient, so the $\mathrm{pH}$ inside the vesicles would be decreased, causing a positive Normalized HPTS value.

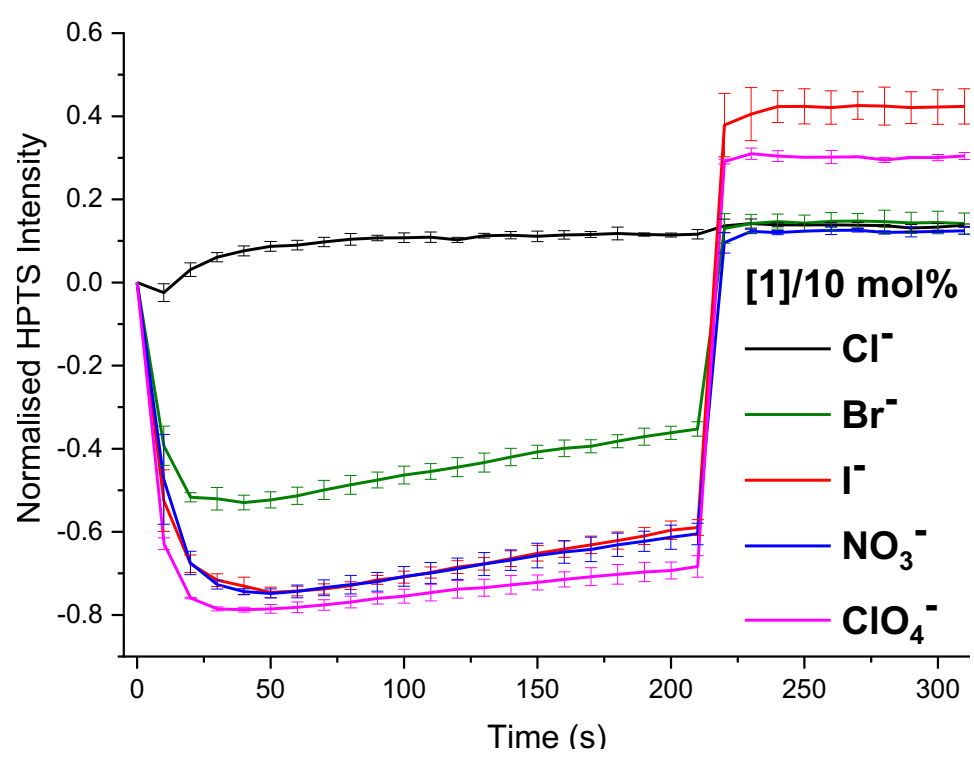

Figure S69: Observed fluorescence ratio response due to $\mathrm{HCl}$ influx in the presence of compounds $1(10 \mathrm{~mol} \%)$ into vesicles loaded with $\mathrm{KCl}(100 \mathrm{mM})$ and suspended in $\mathrm{KCl}, \mathrm{KBr}, \mathrm{KI}$, $\mathrm{KNO}_{3}$ and $\mathrm{KClO}_{4}(100 \mathrm{mM})$. All external and internal solutions were buffered to $\mathrm{pH} 7$ with HEPES $(10 \mathrm{mM})$. 


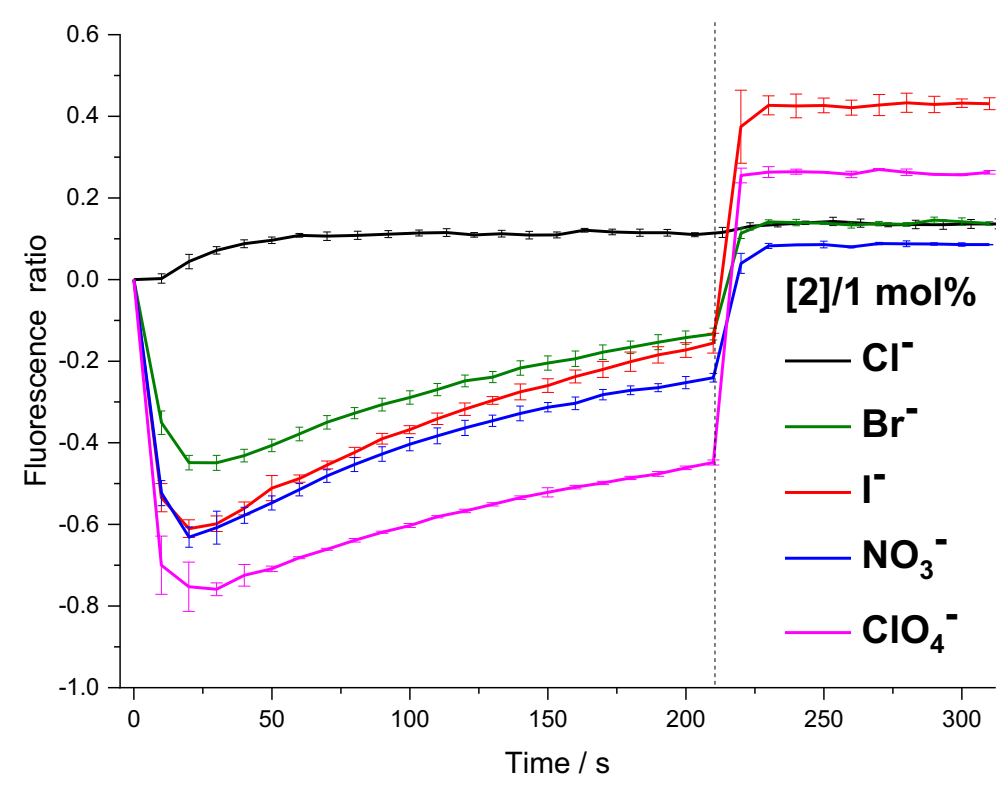

Figure S70: Observed fluorescence ratio response due to $\mathrm{HCl}$ influx in the presence of compounds $2(1 \mathrm{~mol} \%)$ into vesicles loaded with $\mathrm{KCl}(100 \mathrm{mM})$ and suspended in $\mathrm{KCl}, \mathrm{KBr}, \mathrm{KI}$, $\mathrm{KNO}_{3}$ and $\mathrm{KClO}_{4}(100 \mathrm{mM})$. All external and internal solutions were buffered to $\mathrm{pH} 7$ with HEPES (10 mM).

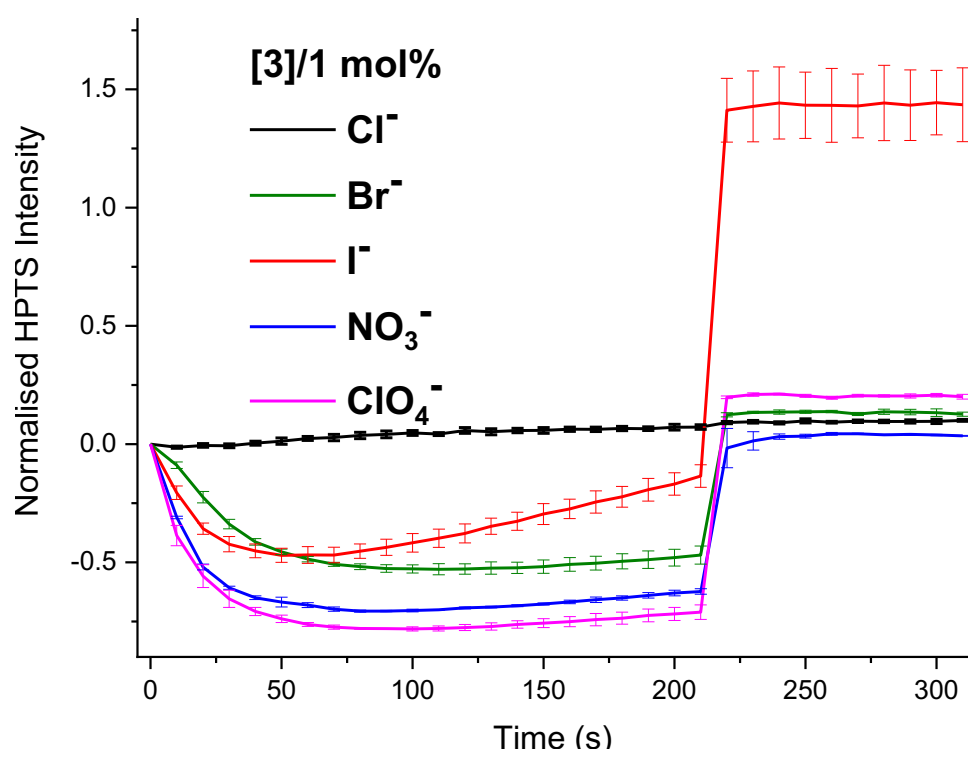

Figure S71: Observed fluorescence ratio response due to $\mathrm{HCl}$ influx in the presence of compounds $3(1 \mathrm{~mol} \%)$ into vesicles loaded with $\mathrm{KCl}(100 \mathrm{mM})$ and suspended in $\mathrm{KCl}, \mathrm{KBr}, \mathrm{KI}$, $\mathrm{KNO}_{3}$ and $\mathrm{KClO}_{4}(100 \mathrm{mM})$. All external and internal solutions were buffered to $\mathrm{pH} 7$ with HEPES $(10 \mathrm{mM})$. 


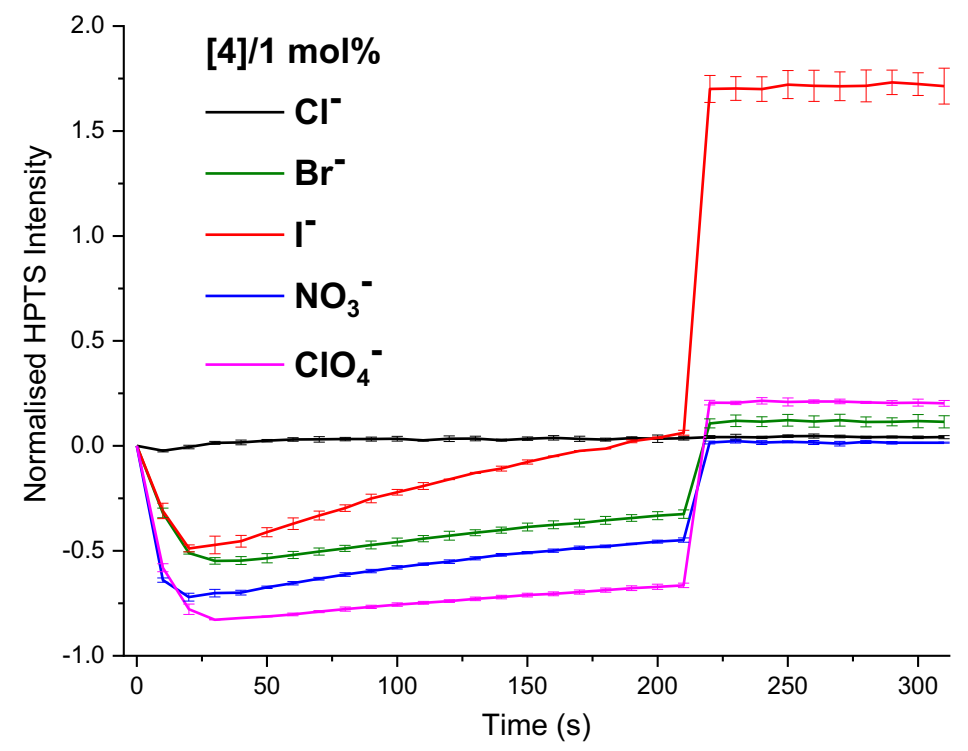

Figure S72: Observed fluorescence ratio response due to $\mathrm{HCl}$ influx in the presence of compounds $4(1 \mathrm{~mol} \%)$ into vesicles loaded with $\mathrm{KCl}(100 \mathrm{mM})$ and suspended in $\mathrm{KCl}, \mathrm{KBr}, \mathrm{KI}$, $\mathrm{KNO}_{3}$ and $\mathrm{KClO}_{4}(100 \mathrm{mM})$. All external and internal solutions were buffered to $\mathrm{pH} 7$ with HEPES $(10 \mathrm{mM})$.

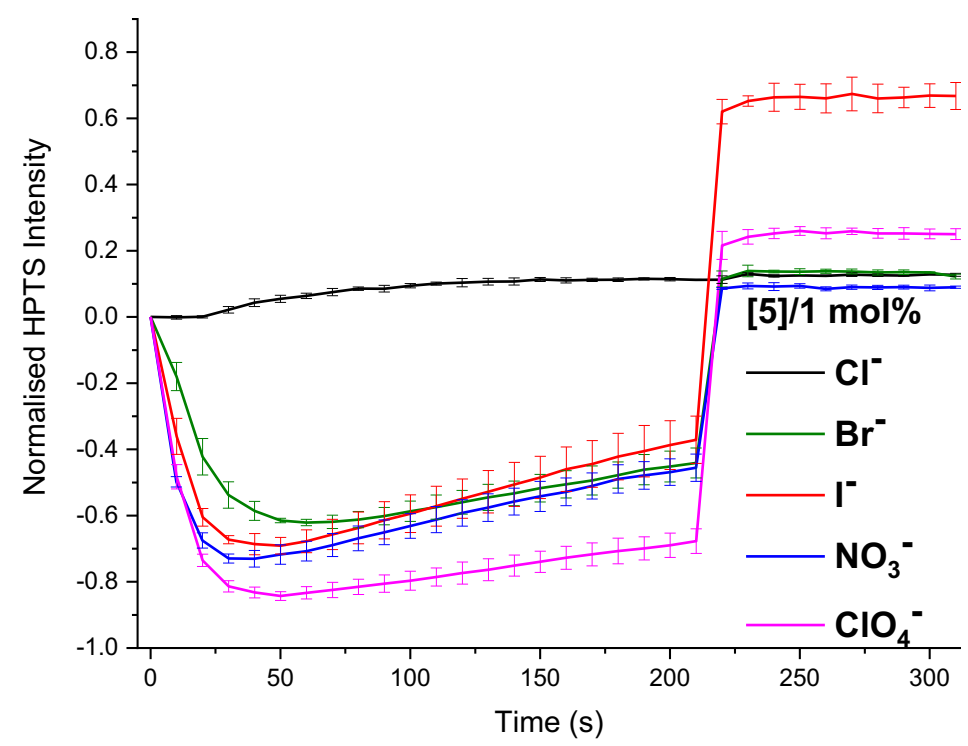

Figure S73: Observed fluorescence ratio response due to $\mathrm{HCl}$ influx in the presence of compounds $5(1 \mathrm{~mol} \%)$ into vesicles loaded with $\mathrm{KCl}(100 \mathrm{mM})$ and suspended in $\mathrm{KCl}, \mathrm{KBr}, \mathrm{KI}$, $\mathrm{KNO}_{3}$ and $\mathrm{KClO}_{4}(100 \mathrm{mM})$. All external and internal solutions were buffered to $\mathrm{pH} 7$ with HEPES $(10 \mathrm{mM})$. 


\section{S6.3: Potassium over sodium selectivity:}

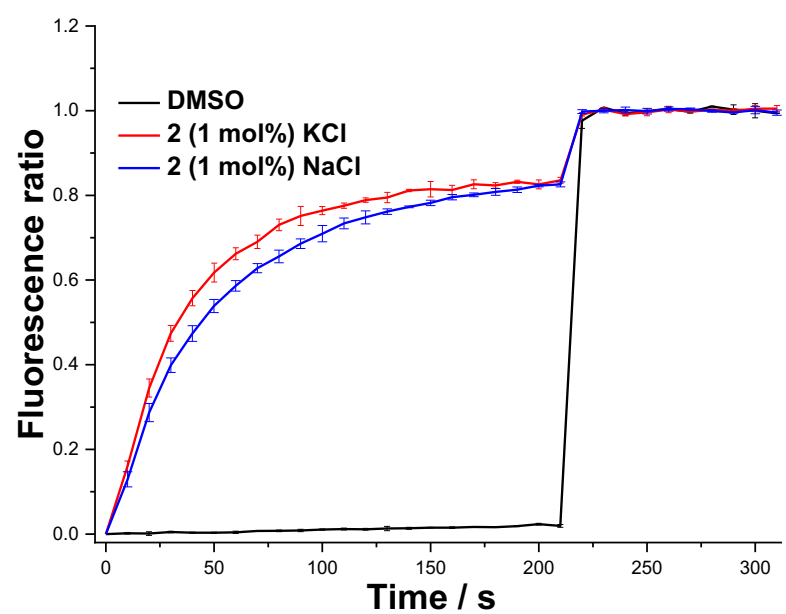

Figure S74: Observed fluorescence ratio response due to $\mathrm{HCl}$ influx or $\mathrm{Cl}^{-} / \mathrm{OH}^{-}$antiport facilitated by compound $2(1 \mathrm{~mol} \%)$ using $\mathrm{KCl}-\mathrm{KOH}$ and $\mathrm{NaCl}-\mathrm{KOH}$ assays from POPC vesicles loaded with $\mathrm{KCl}(100 \mathrm{mM})$ or $\mathrm{NaCl}$, respectively. All external and internal solutions were buffered to $\mathrm{pH} 7.0$ with HEPES $(10 \mathrm{mM})$. The test compound was added at $0 \mathrm{~s}$ and detergent was added at $200 \mathrm{~s}$. Ionophore concentrations are shown as ionophore to lipid molar ratios. Error bars represent SD from at least three repeats.

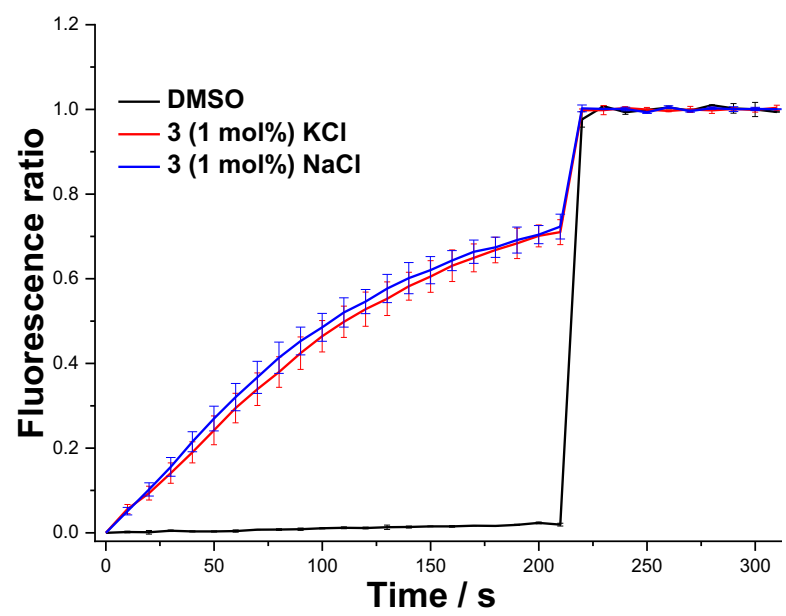

Figure S75: Observed fluorescence ratio response due to $\mathrm{HCl}$ influx or $\mathrm{Cl}^{-} / \mathrm{OH}^{-}$antiport facilitated by compound $3(1 \mathrm{~mol} \%)$ using $\mathrm{KCl}-\mathrm{KOH}$ and $\mathrm{NaCl}-\mathrm{KOH}$ assays from POPC vesicles loaded with $\mathrm{KCl}(100 \mathrm{mM})$ or $\mathrm{NaCl}$, respectively. All external and internal solutions were buffered to $\mathrm{pH} 7.0$ with HEPES $(10 \mathrm{mM})$. The test compound was added at $0 \mathrm{~s}$ and detergent was added at $200 \mathrm{~s}$. Ionophore concentrations are shown as ionophore to lipid molar ratios. Error bars represent SD from at least three repeats. 


\section{S6.4: Calcein leakage assays:}

A chloroform solution of POPC was evaoprated under vacuum and dried for at least $6 \mathrm{~h}$ as reported. ${ }^{[9]}$ The thin film was hydrated by the internal solution containing calcein disodium salt $(100 \mathrm{mM})$ and $\mathrm{NaCl}(100 \mathrm{mM})$ buffered to $\mathrm{pH} 7.4$ with HEPES (10 mM). Then, the lipid suspension was subjected to nine freeze-thaw cycles followed by extrusion 25 times through a $200 \mathrm{~nm}$ polycarbonate membrane. Size exclusion chromatography using sephadex G-25 column and calcein-free external solution, containing $\mathrm{NaCl}$ (100 $\mathrm{mM}$ ) and $\mathrm{Na}_{2} \mathrm{SO}_{4}(100 \mathrm{mM})$ buffered to $\mathrm{pH} 7.4$ with HEPES $(10 \mathrm{mM})$.

The resulting suspension of dye-encapsulated LUVs with a mean diameter of $200 \mathrm{~nm}$ was diluted with the external solution to obtain $2.5 \mathrm{~mL}$ lipid suspension containing a 0.1 $\mathrm{mM}$ lipid concentration. After the tested receptors 1-5 were added at $1 \mathrm{~mol} \%$, calcein fluorescence $\left(\lambda_{\mathrm{ex}}=490 \mathrm{~nm}, \lambda_{\mathrm{em}}=520 \mathrm{~nm}\right)$ was recorded at $25^{\circ} \mathrm{C}$. Detergent $(25 \mu \mathrm{L})$ was added at 200 seconds to lyse the vesicle and to calibrate the assay.

The fractional calcein release $(\mathrm{FR})$ was calculated as follows (with $I_{t}=$ fluorescence intensity at time $\mathrm{t}, I_{0}=$ fluorescence intensity at time 0 and $I_{\max }=$ fluorescence intensity after addition of detergent):

$$
F R=\frac{I_{t}-I_{0}}{I_{\max }-I_{o}}
$$




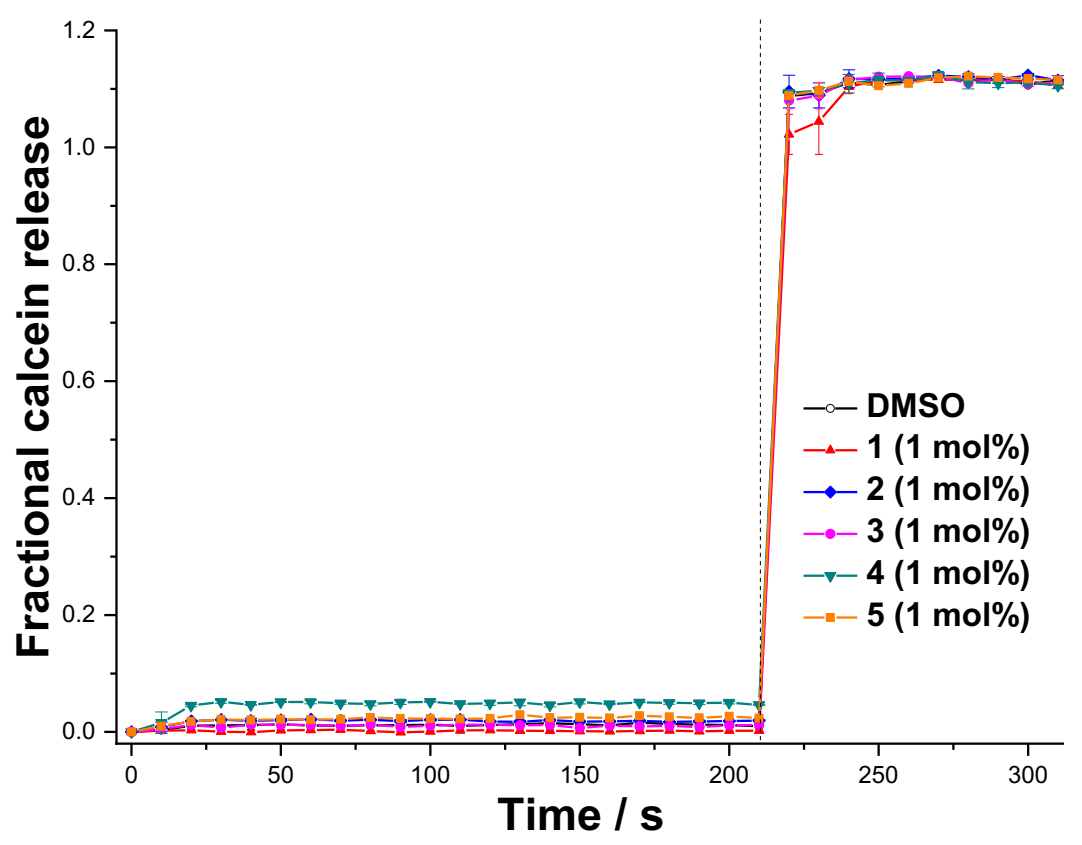

Figure S76: Calcein leakage by 1-5 (1 mol\% carrier-to-lipid) from unilamellar POPC vesicles loaded with calcein disodium salt $(100 \mathrm{mM})$ and $\mathrm{NaCl}(100 \mathrm{mM})$ buffered to $\mathrm{pH} 7.4$ with HEPES $(10 \mathrm{mM})$. At $\mathrm{t}=10 \mathrm{~s}$, a DMSO solution of the transporter was added to start the experiment. At the end of the experiment, detergent was added to lyse the vesicles. The results are shown as the fraction of calcein leaked from the vesicles. 


\section{S6.5: Ion selective electrode (ISE) assays:}

a- $\mathrm{Cl} / \mathrm{NO}_{3}$ exchange assay:

Unilamellar vesicles were prepared as reported. ${ }^{[8]}$ Briefly, $~ 30 \mathrm{mg}$ of POPC (1-palmitoyl2-oleoylphosphatidylcholine) was dissolved in $1 \mathrm{~mL}$ chloroform in a round-bottomed flask and the solvent was removed in vacuo to form a thin lipid layer. The thin film was dried under high vacuum for at least $6 \mathrm{~h}$ and suspended on the internal solution $(4 \mathrm{~mL})$ and vortexed using a lab dancer to form large multilamellar vesicles, which was subjected to nine freeze-thaw cycles alternating between water (at room temperature) and liquid nitrogen. Further, the formed lipid was left to rest for 30 minutes and then subjected to extrusion through a $200 \mathrm{~nm}$ polycarbonate membranes 25 times to form the unilamellar vesicles. The formed vesicles were subjected to dialysis for $4 \mathrm{~h}$ in the desired external solution to remove any unencapsulated internal salts. Finally, using the required external solution, the lipid was diluted to $1.0 \mathrm{mM}$. The $\mathrm{pH}$ of the internal and external solutions was maintained at 7.2 using phosphate buffer with a $500 \mathrm{mM}$ total ionic strength. Test compound in DMSO $(40 \mu \mathrm{L})$ was added to start the experiment and chloride selective electrode was used to monitor the chloride efflux. Detergent ( $50 \mu \mathrm{L})$ was added after 300 seconds to lyse the vesicles, while the $100 \%$ chloride efflux reading was taken at 420 seconds.

- Hill plots for $\mathrm{Cl} / \mathrm{NO}_{3}$ :

Hill plots were performed for $\mathrm{Cl} / \mathrm{NO}_{3}$ exchange assay by conducting transport assay at different concentrations of tested compounds. Receptor concentration vs chloride efflux at $270 \mathrm{~s}$ (the endpoint of transport assay) were plotted and fitted to the Hill equation using Origin 2019b:

$$
y=V_{\max } \frac{x^{n}}{k^{n}+x^{n}}=100 \% \frac{x^{n}}{\left(E C_{50}\right)^{n}+x^{n}}
$$

Where $y$ is the chloride efflux at $270 \mathrm{~s}(\%)$ and $\mathrm{x}$ is the tested compound concentration (mol\% relative to lipid concentration)

$V_{\max }$ is the maximum efflux possible and considered as $100 \%$ as this is experimentally the maximum chloride efflux possible.

$k\left(E C_{50}\right.$ value $)$ is the carrier concentration needed to reach $V_{\max } / 2$. 
Each data point on each Hill plot are an average of at least two repeated runs. Error bars represent standard deviation about the mean.

Hill plots for receptors $\mathbf{1}$ and $\mathbf{5}$ were not performed due to low potency and solubility issues of ionophore 1 and 5 , respectively.

b- $\mathrm{KCl}$ efflux - cationohore coupling:

In this assay, $300 \mathrm{mM}$ total ionic strength of both $\mathrm{K}$ gluconate external solution and $\mathrm{KCl}$ internal solution were marinated. The vesicles were made in a similar way to $\mathrm{Cl} / \mathrm{NO}_{3}$ exchange assay except that gel filtration, using sephadex, replaced dialysis to allow exchange of any unencapsulated $\mathrm{KCl}$ for KGlu. External KGlu solution (10 mL) was used to dilute the lipid solution obtained after sephadex to obtain a lipid stock of known concentration. A cationohore, monensin or Valinomycin, $(10 \mu \mathrm{L}, 0.5 \mathrm{mM})$ was added first to the lipid solution at concentration $0.1 \mathrm{~mol} \%$ with respect to lipid concentration. Then, receptor was added after 30 seconds of the cationohore addition to start the experiment. 


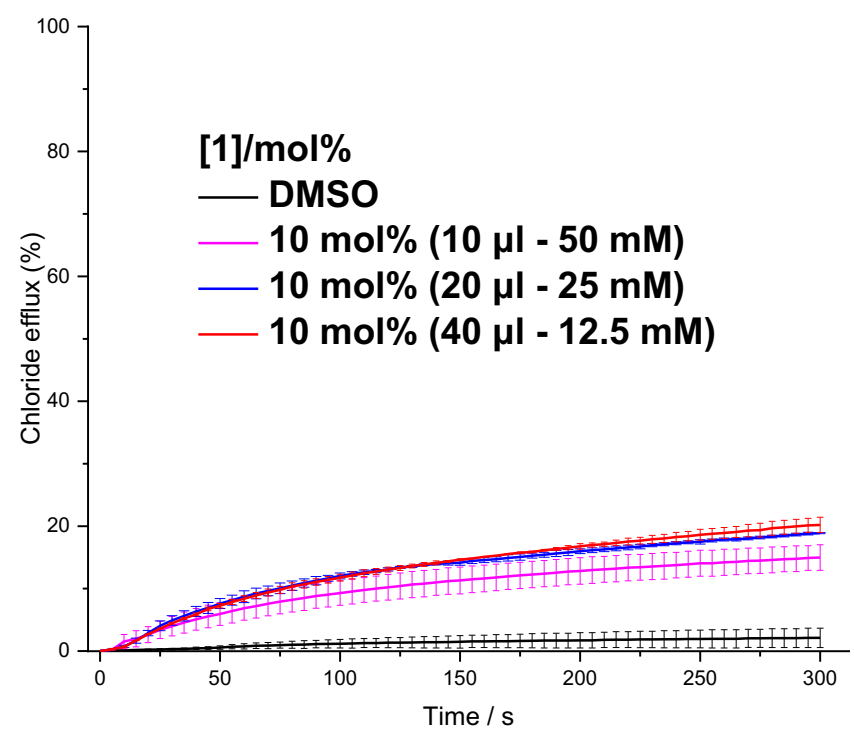

Figure S77: Chloride efflux mediated by receptor 1 (10 mol\% w.r.t. lipid), added from stock DMSO solutions of the receptor at varying concentration with different loading volume. The compound was added to POPC vesicles containing $489 \mathrm{mM} \mathrm{KCl}$ and suspended in $489 \mathrm{mM} \mathrm{KNO}$, both buffered to $\mathrm{pH} 7.2$ with phosphate buffer. At the end of the experiment the vesicles were lysed with detergent to achieve $100 \%$ chloride efflux. Each point represents the average of 3 trials.

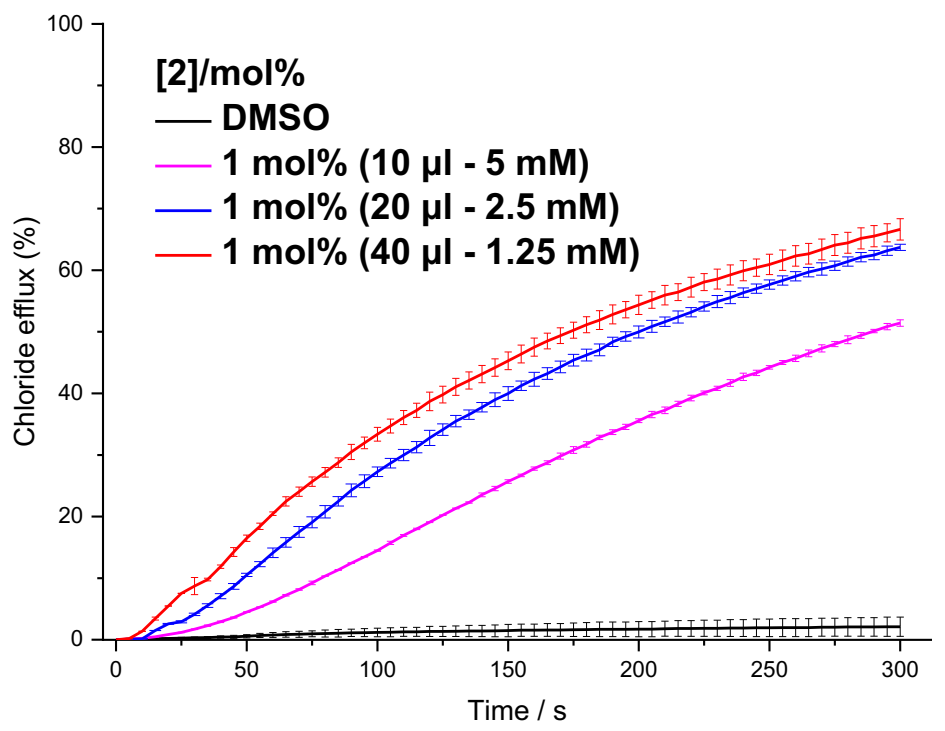

Figure S78: Chloride efflux mediated by receptor 2 ( $1 \mathrm{~mol} \%)$, added from stock DMSO solutions of the receptor at varying concentration with different loading volume. The compound was added to POPC vesicles containing $489 \mathrm{mM} \mathrm{KCl}$ and suspended in $489 \mathrm{mM} \mathrm{KNO}_{3}$, both buffered to $\mathrm{pH}$ 7.2 with phosphate buffer. At the end of the experiment the vesicles were lysed with detergent to achieve $100 \%$ chloride efflux. Each point represents the average of 3 trials. 


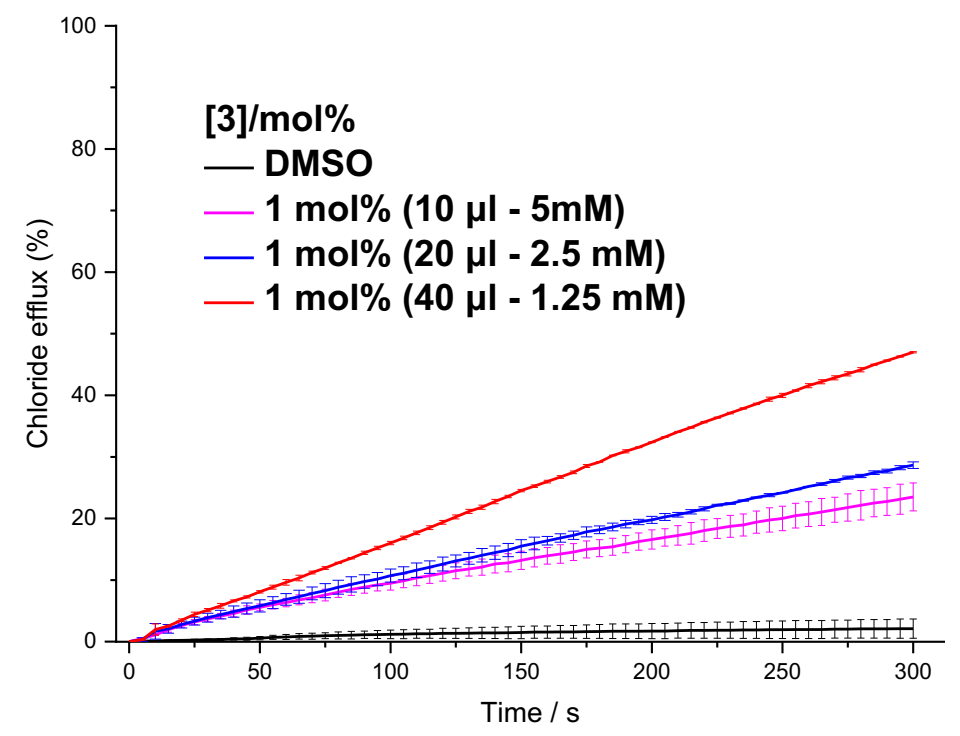

Figure S79: Chloride efflux mediated by receptor 3 (1 mol\%), added from stock DMSO solutions of the receptor at varying concentration with different loading volume. The compound was added to POPC vesicles containing $489 \mathrm{mM} \mathrm{KCl}$ and suspended in $489 \mathrm{mM} \mathrm{KNO}_{3}$, both buffered to $\mathrm{pH}$ 7.2 with phosphate buffer. At the end of the experiment the vesicles were lysed with detergent to achieve $100 \%$ chloride efflux. Each point represents the average of 3 trials.

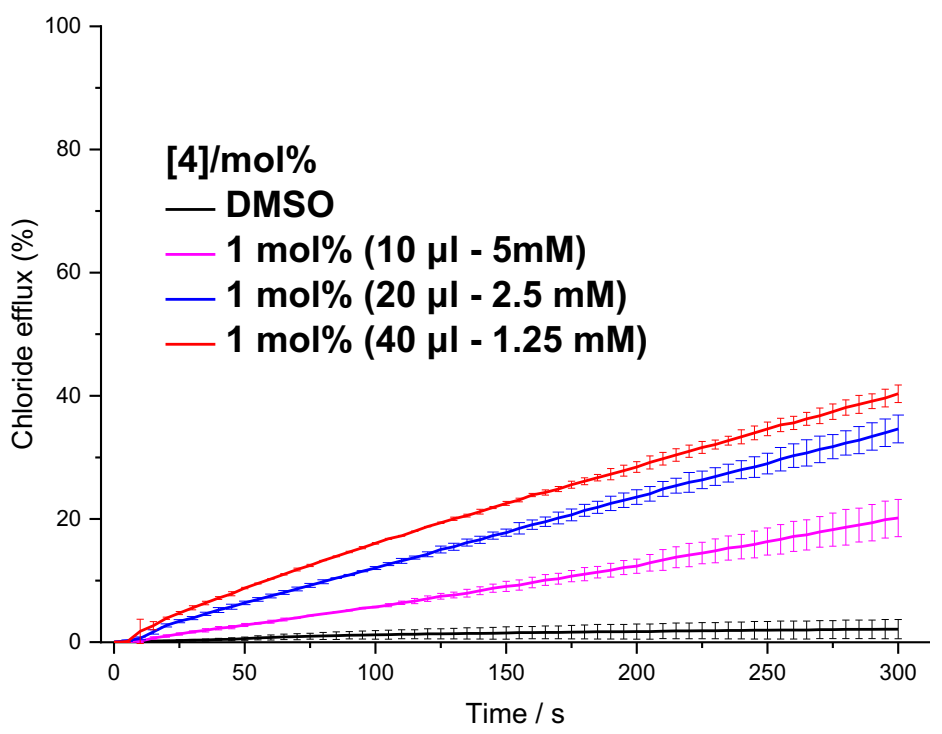

Figure S80: Chloride efflux mediated by receptor 4 (1 mol\%), added from stock DMSO solutions of the receptor at varying concentration with different loading volume. The compound was added to POPC vesicles containing $489 \mathrm{mM} \mathrm{KCl}$ and suspended in $489 \mathrm{mM} \mathrm{KNO}_{3}$, both buffered to $\mathrm{pH}$ 7.2 with phosphate buffer. At the end of the experiment the vesicles were lysed with detergent to achieve $100 \%$ chloride efflux. Each point represents the average of 3 trials. 

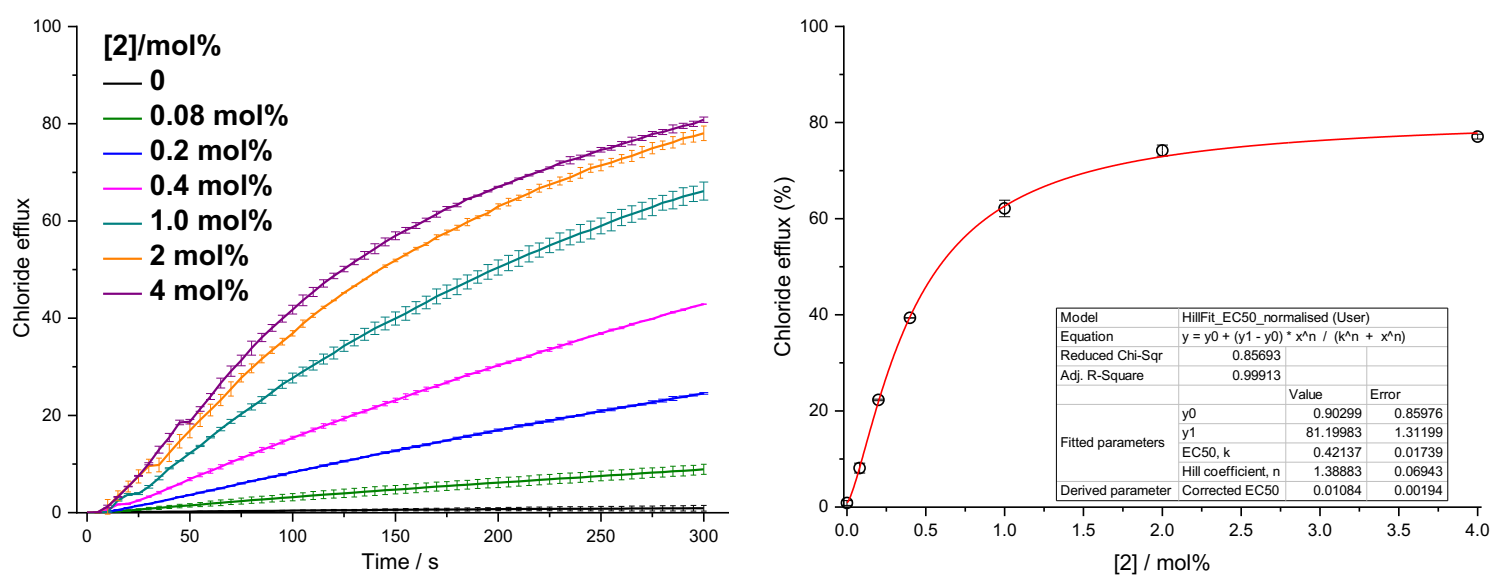

Figure S81: Hill plot analysis of chloride efflux facilitated by compound $\mathbf{2}$ from unilamellar POPC vesicles that were loaded with a $489 \mathrm{mM} \mathrm{KCl}$ solution buffered to $\mathrm{pH} 7.2$ with $5 \mathrm{mM}$ phosphate, and were suspended in a $489 \mathrm{mM} \mathrm{KNO}_{3}$ solution buffered to $\mathrm{pH} 7.2$ with $5 \mathrm{mM}$ phosphate salts. DMSO was used as a control. Each point is the average of three repeats.
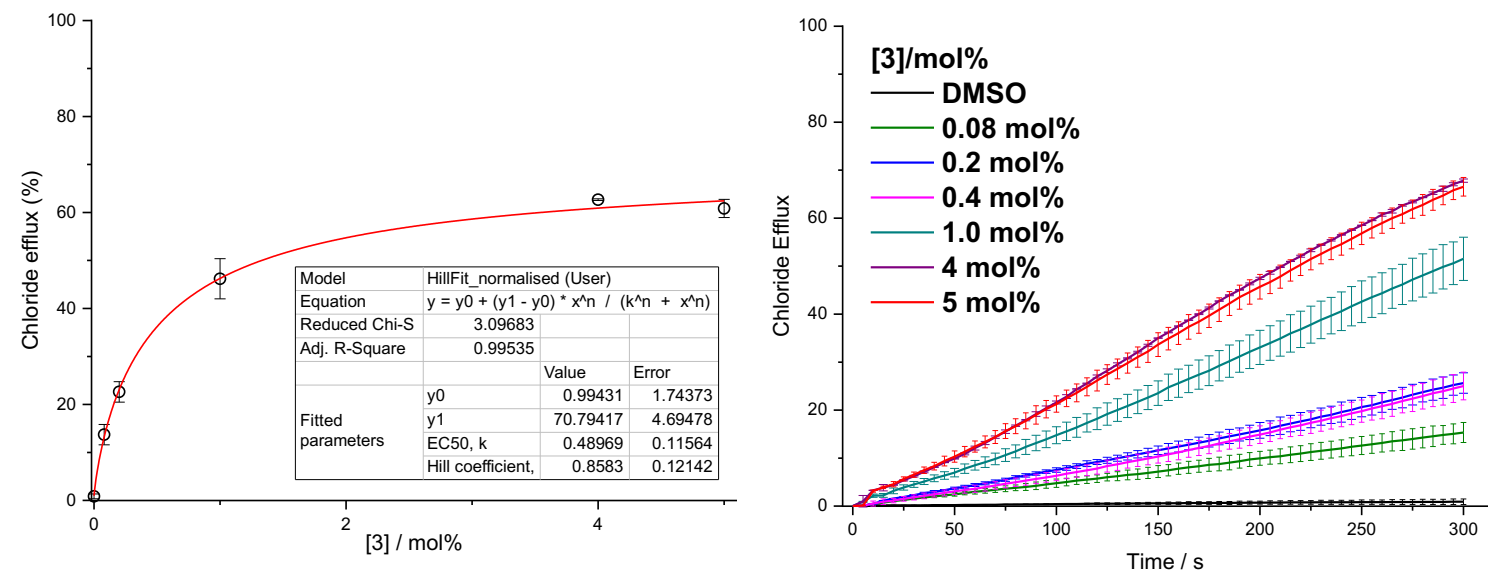

Figure S82: Hill plot analysis of chloride efflux facilitated by compound 3 from unilamellar POPC vesicles that were loaded with a $489 \mathrm{mM} \mathrm{KCl}$ solution buffered to $\mathrm{pH} 7.2$ with $5 \mathrm{mM}$ phosphate, and were suspended in a $489 \mathrm{mM} \mathrm{KNO}_{3}$ solution buffered to $\mathrm{pH} 7.2$ with $5 \mathrm{mM}$ phosphate salts. DMSO was used as a control. Each point is the average of three repeats. 

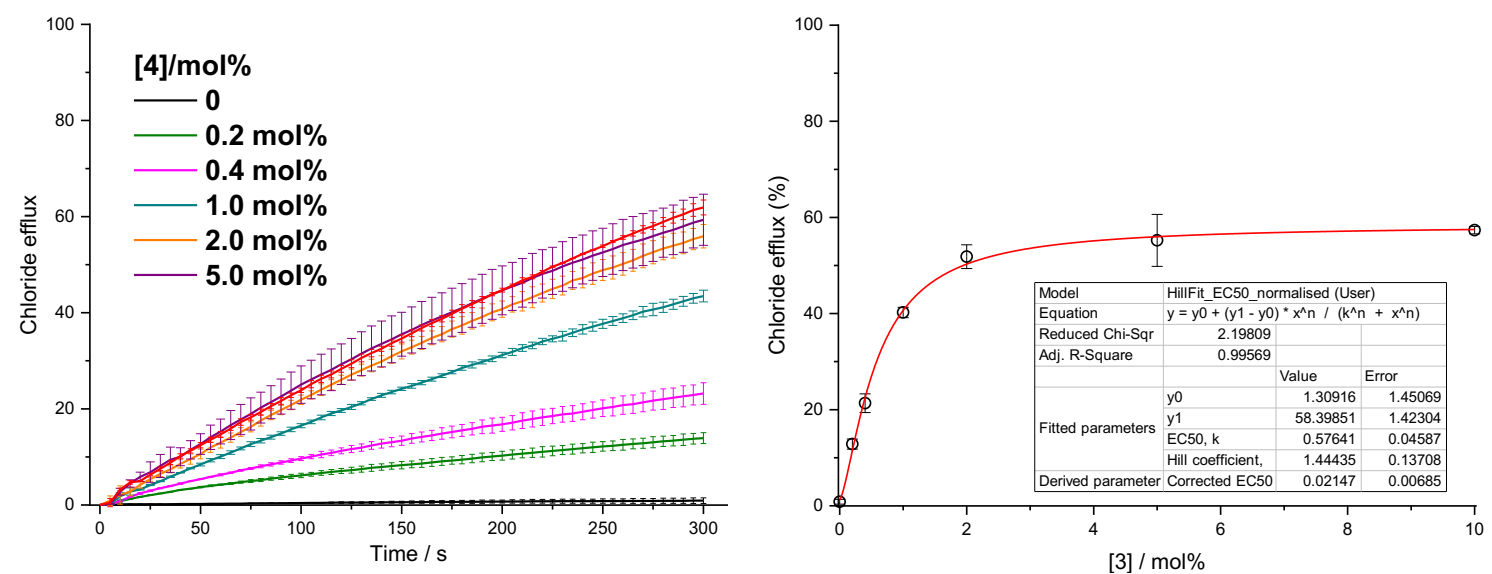

Figure S83: Hill plot analysis of chloride efflux facilitated by compound $\mathbf{4}$ from unilamellar POPC vesicles that were loaded with a $489 \mathrm{mM} \mathrm{KCl}$ solution buffered to $\mathrm{pH} 7.2$ with $5 \mathrm{mM}$ phosphate, and were suspended in a $489 \mathrm{mM} \mathrm{KNO}_{3}$ solution buffered to $\mathrm{pH} 7.2$ with $5 \mathrm{mM}$ phosphate salts. DMSO was used as a control. Each point is the average of three repeats.

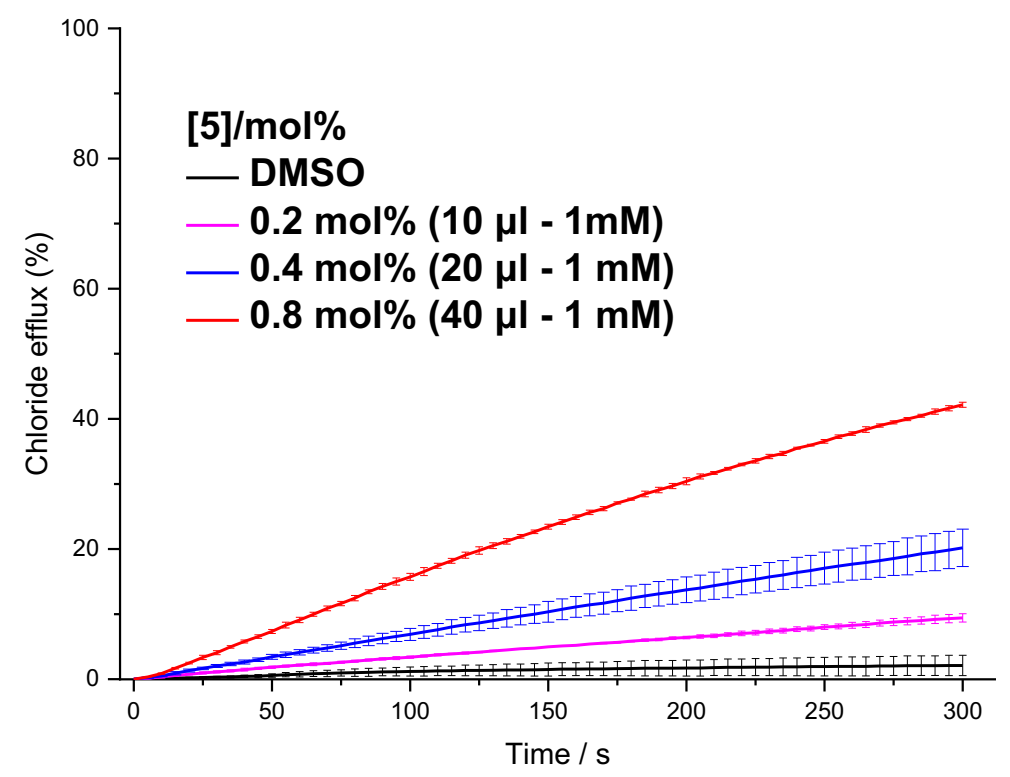

Figure S84: Chloride efflux mediated by different mol\% of transporter $\mathbf{5}$, from unilamellar POPC vesicles that were loaded with a $489 \mathrm{mM} \mathrm{KCl}$ solution buffered to $\mathrm{pH} 7.2$ with $5 \mathrm{mM}$ phosphate, and were suspended in a $489 \mathrm{mM} \mathrm{KNO}_{3}$ solution buffered to $\mathrm{pH} 7.2$ with $5 \mathrm{mM}$ phosphate salts. DMSO was used as a control. Each point is the average of three repeats. The maximim observed solubility of compound 5 in DMSO was $1 \mathrm{mM}$, so higher concentration could not be performed to get hill plot. 


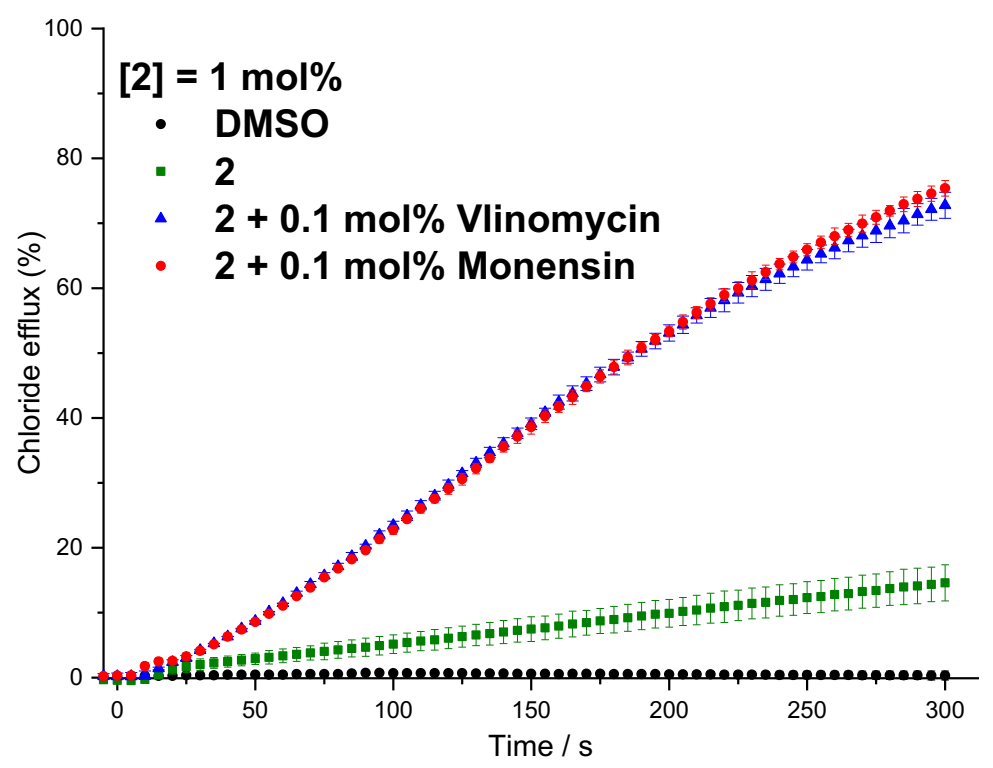

Figure S85: Electrogenic or electroneutral transport mediated by compound 2 ( $1 \mathrm{~mol} \%$ with respect to lipid) in the presence of monensin or valinomycin $(0.1 \mathrm{~mol} \%$ with respect to lipid). Unilamellar POPC vesicles were loaded with a $300 \mathrm{mM} \mathrm{KCl}$ solution buffered to $\mathrm{pH} 7.2$ with $5 \mathrm{mM}$ phosphate and were suspended in a $300 \mathrm{mM}$ KGlu solution buffered to $\mathrm{pH} 7.2$ with $5 \mathrm{mM}$ phosphate salts. DMSO was used as a control. Each point is the average of three repeats.

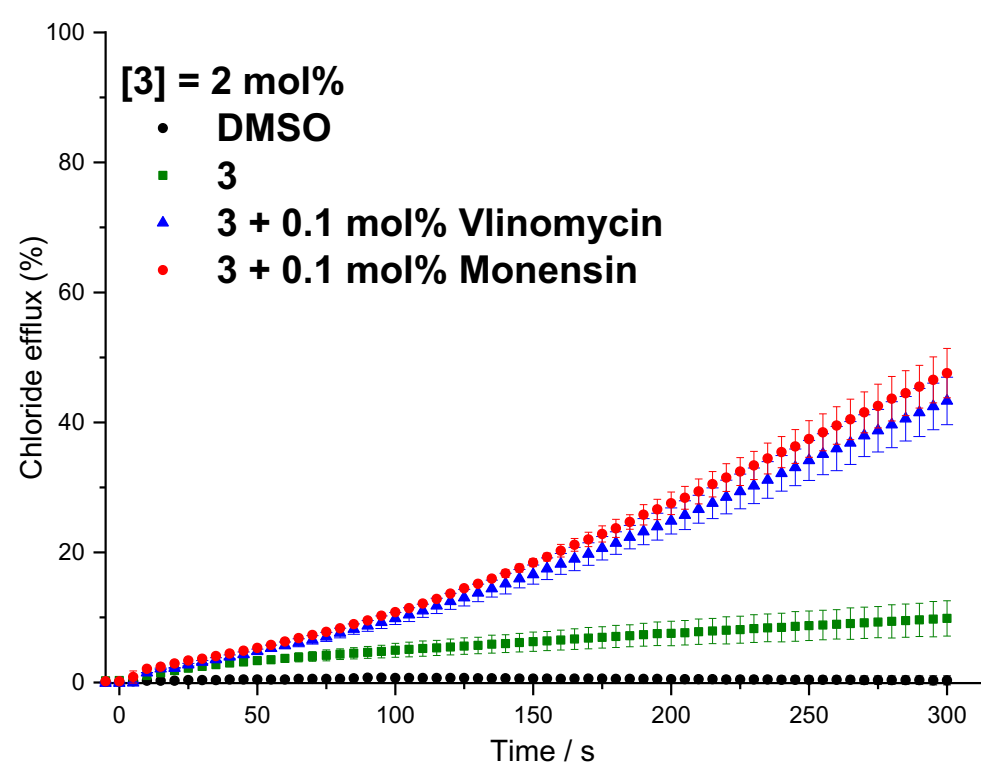

Figure S86: Electrogenic or electroneutral transport mediated by compound 3 ( $1 \mathrm{~mol} \%$ with respect to lipid) in the presence of monensin or valinomycin $(0.1 \mathrm{~mol} \%$ with respect to lipid). Unilamellar POPC vesicles were loaded with a $300 \mathrm{mM} \mathrm{KCl}$ solution buffered to $\mathrm{pH} 7.2$ with $5 \mathrm{mM}$ phosphate and were suspended in a $300 \mathrm{mM}$ KGlu solution buffered to $\mathrm{pH} 7.2$ with $5 \mathrm{mM}$ phosphate salts. DMSO was used as a control. Each point is the average of three repeats. 


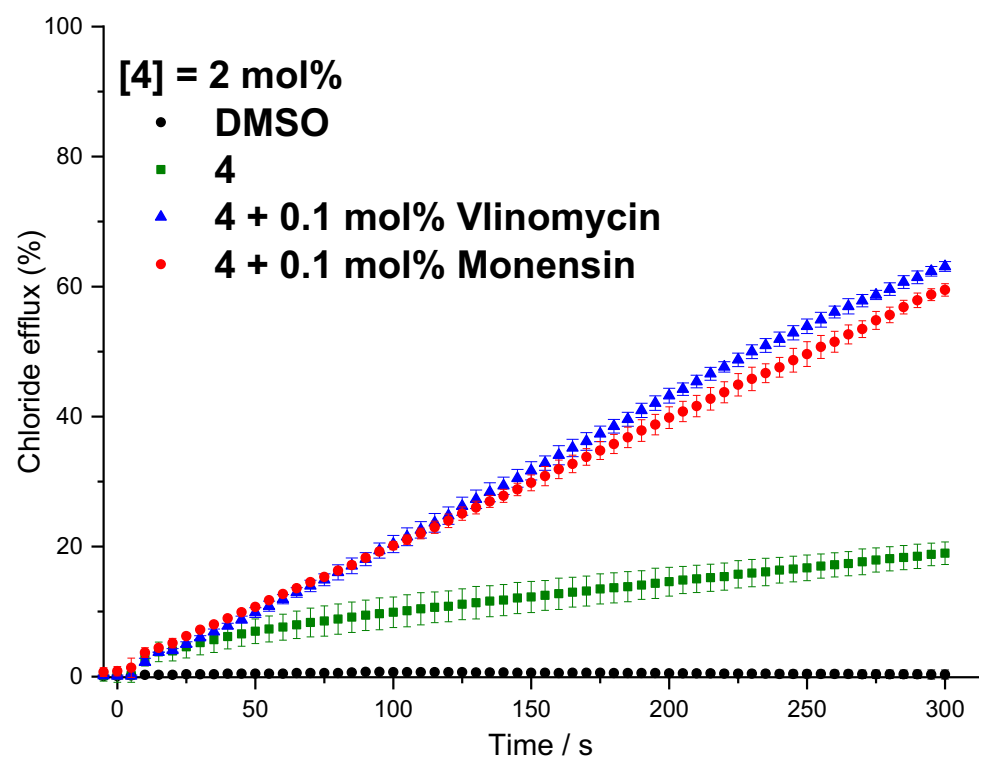

Figure S87: Electrogenic or electroneutral transport mediated by compound 4 (1 mol\% with respect to lipid) in the presence of monensin or valinomycin $(0.1 \mathrm{~mol} \%$ with respect to lipid). Unilamellar POPC vesicles were loaded with a $300 \mathrm{mM} \mathrm{KCl}$ solution buffered to $\mathrm{pH} 7.2$ with $5 \mathrm{mM}$ phosphate and were suspended in a $300 \mathrm{mM}$ KGlu solution buffered to $\mathrm{pH} 7.2$ with $5 \mathrm{mM}$ phosphate salts. DMSO was used as a control. Each point is the average of three repeats. 


\section{S6.6: Fluorescence titration studies with TBACI}

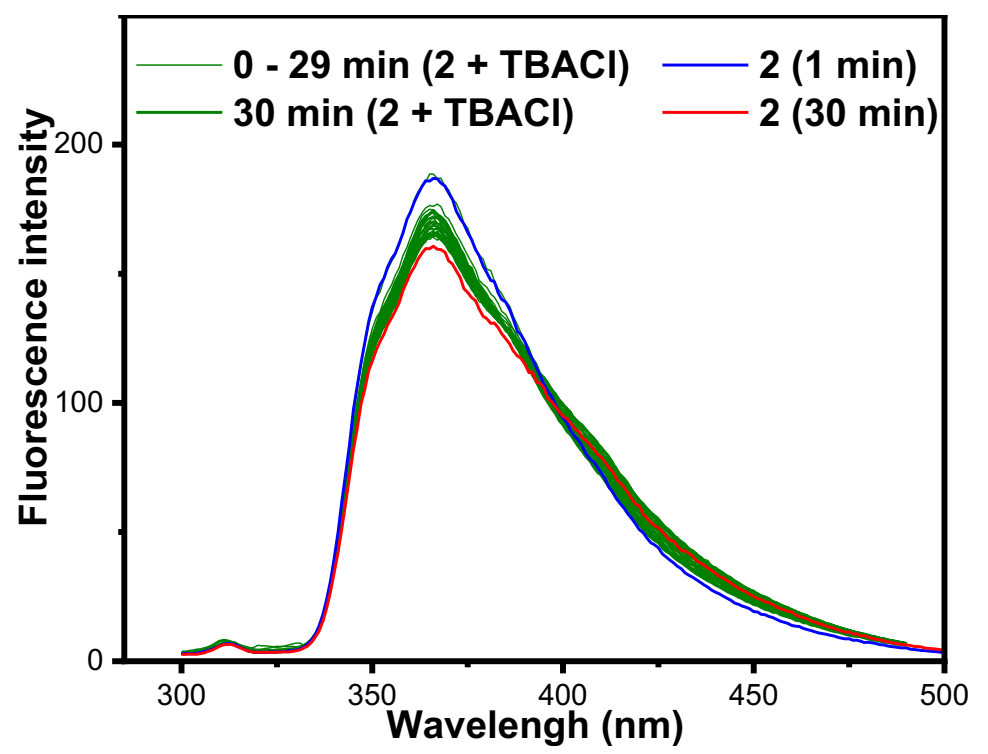

Figure S88: Fluorescent changes over 30 minutes $(E x=285 \mathrm{~nm})$ of compound $2(1.0 \mu \mathrm{M})$ upon addition of 100 equivalents of TBACl $(100 \mu \mathrm{M})$ in DMSO.

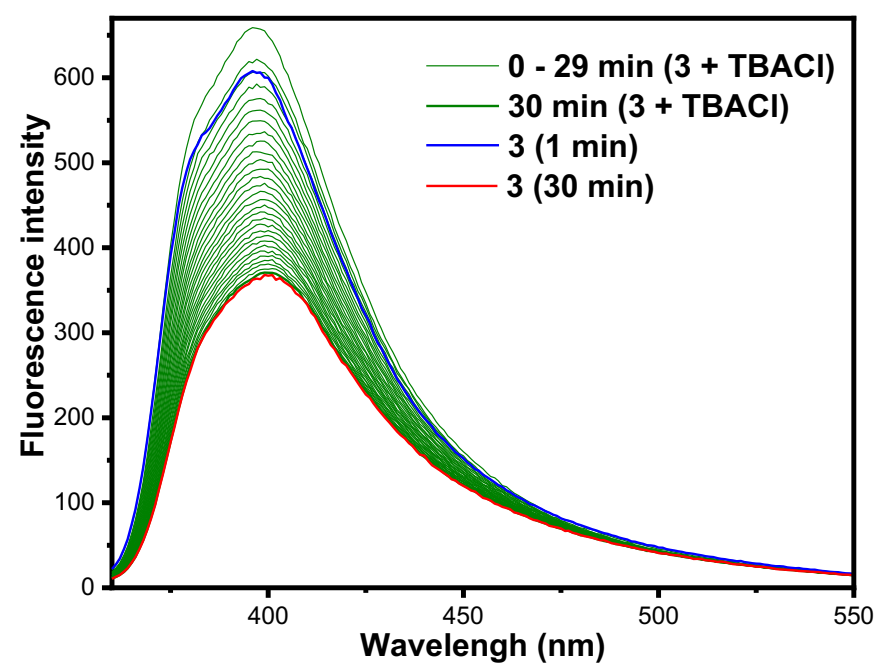

Figure S89: Fluorescent changes over 30 minutes $(E x=350 \mathrm{~nm})$ of compound $3(1.0 \mu \mathrm{M})$ upon addition of 100 equivalents of TBACl $(100 \mu \mathrm{M})$ in DMSO. 


\section{S7: ${ }^{1} \mathrm{H}$ NMR Titration Binding Studies with TBACI}

Proton NMR titrations binding studies were performed on Bruker $400 \mathrm{MHz}$ FT-NMR spectrometer at $298 \mathrm{~K}$. Solution of receptors $1-4$ in DMSO- $d_{6} / 0.5 \% \mathrm{H}_{2} \mathrm{O}$ were prepared in $2 \mathrm{mM}$. The guest anion, tetra- $n$-butylammonium (TBA) chloride, was prepared with the same receptor solution, to ensure the overall receptor concentration stays constant whilst the guest anion concentration changes. Using small aliquots of the guest salt, the receptor solution was titrated, and after each addition, chemical shifts were reported in ppm in refence to residual solvent peaks. The isotherm based on the $\mathrm{NH}$ and $\mathrm{CH}$ chemical shifts were globally fitted using the online fitting program Bindfit. ${ }^{[10]}$ 

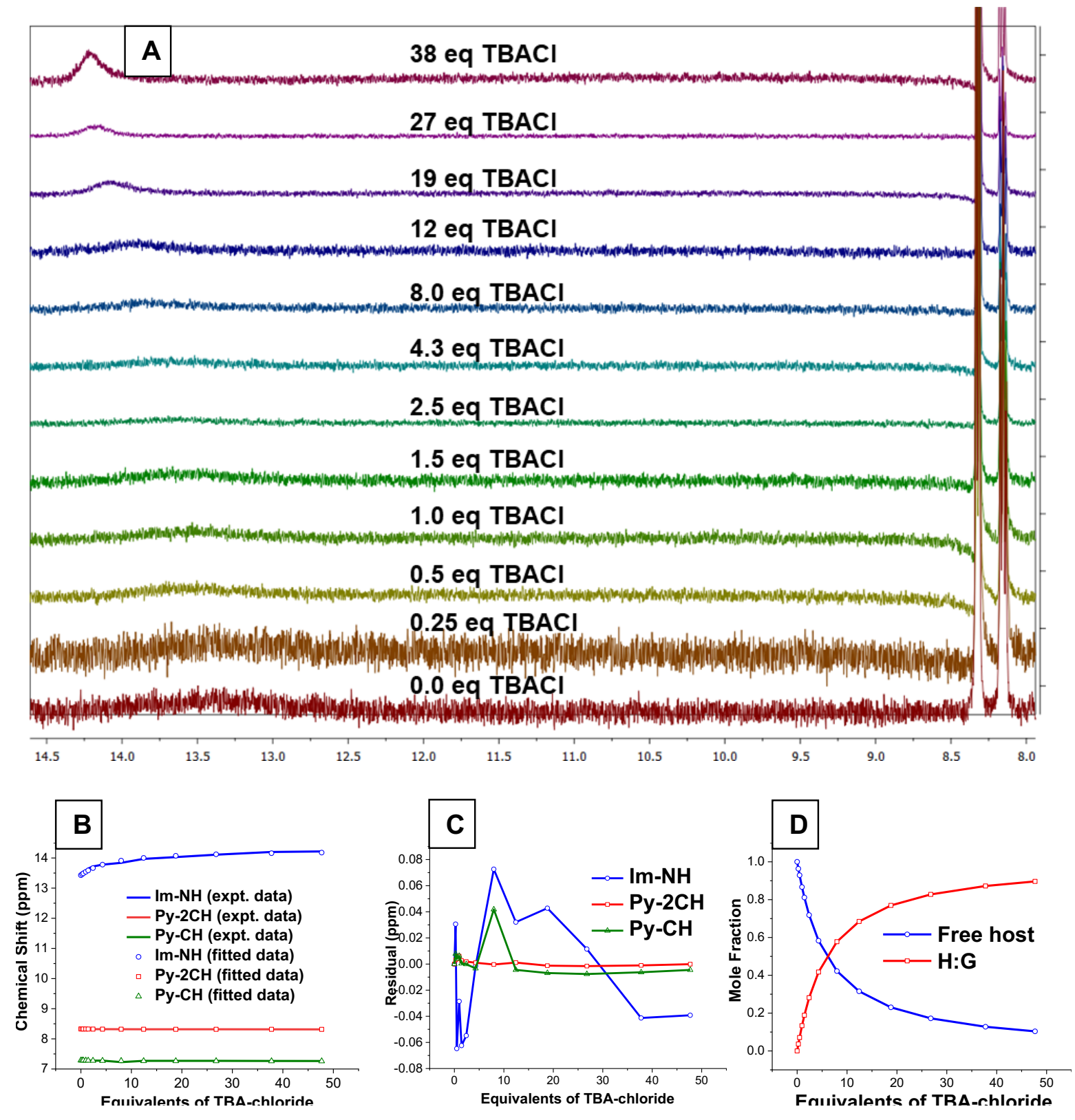

Figure S90: ${ }^{1} \mathrm{H}$ NMR spectroscopic titration of receptor $1(2 \mathrm{mM})$ with TBACl in DMSO- $d_{6}$ with $\mathbf{0 . 5 \%}$ water at $298 \mathrm{~K}$. (a) Stack plot. (b) Fitplot for $\mathrm{NH}$ proton at $\delta=13.43 \mathrm{ppm}$ (proton1, Im-NH) and $\mathrm{CH}$ protons at $\delta=8.33 \mathrm{ppm}$ (proton2, $\mathrm{Py}-2 \mathrm{CH}$ ) and $\delta=7.29 \mathrm{ppm}$ (proton3, $\mathrm{Py}-\mathrm{CH}$ ), using global analysis with $1: 1\left(\mathrm{~K}_{\mathrm{a}}=92 \mathrm{M}^{-1}\right.$, error: $\left.13 \%\right)$. (c) Plot of the residuals for $\delta=13.43 \mathrm{ppm}$ (proton1), $\delta=8.33 \mathrm{ppm}$ (proton2) and $\delta=7.29 \mathrm{ppm}$ (proton3) using global analysis. (d) Calculated mole fractions. 


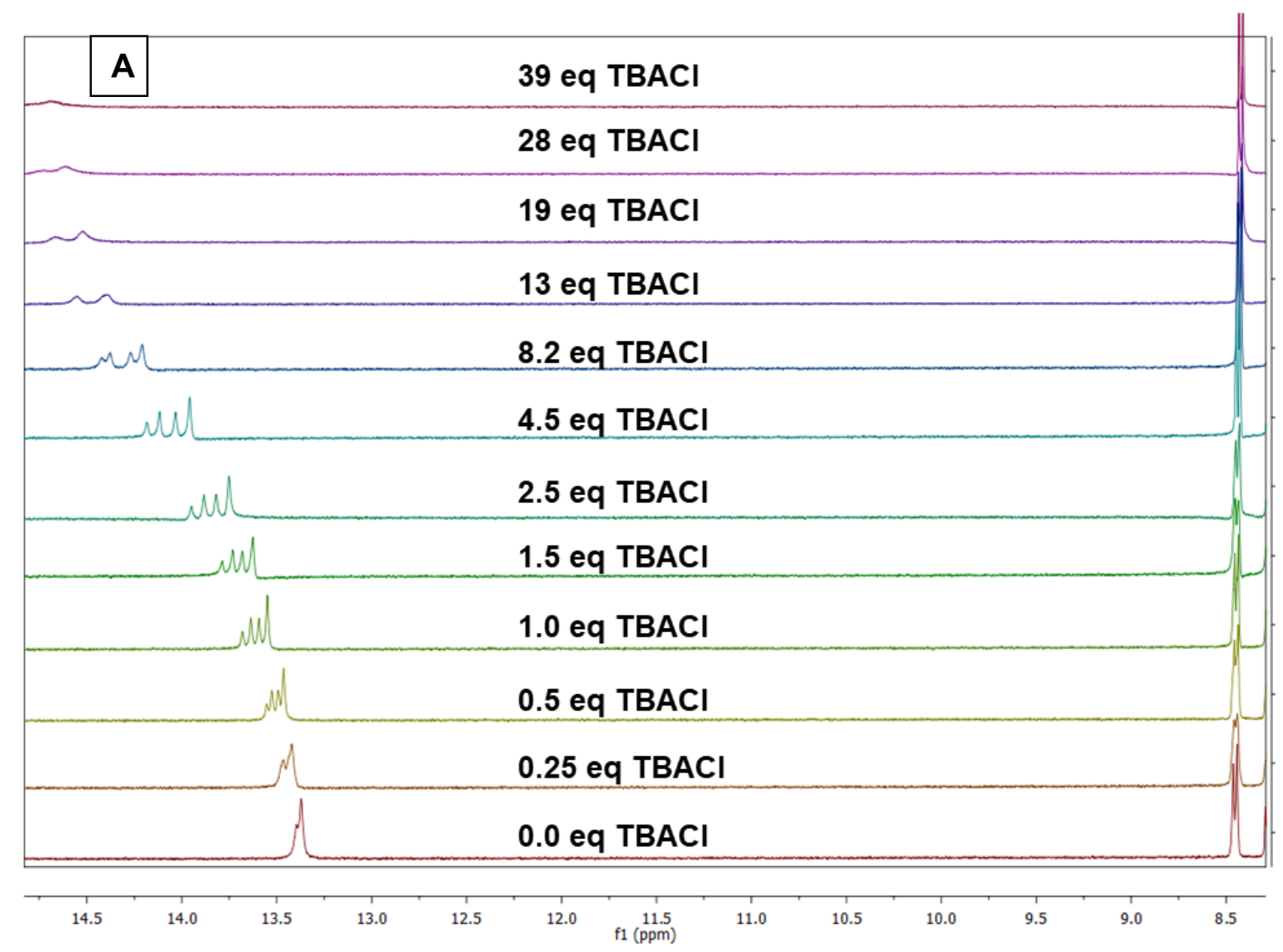
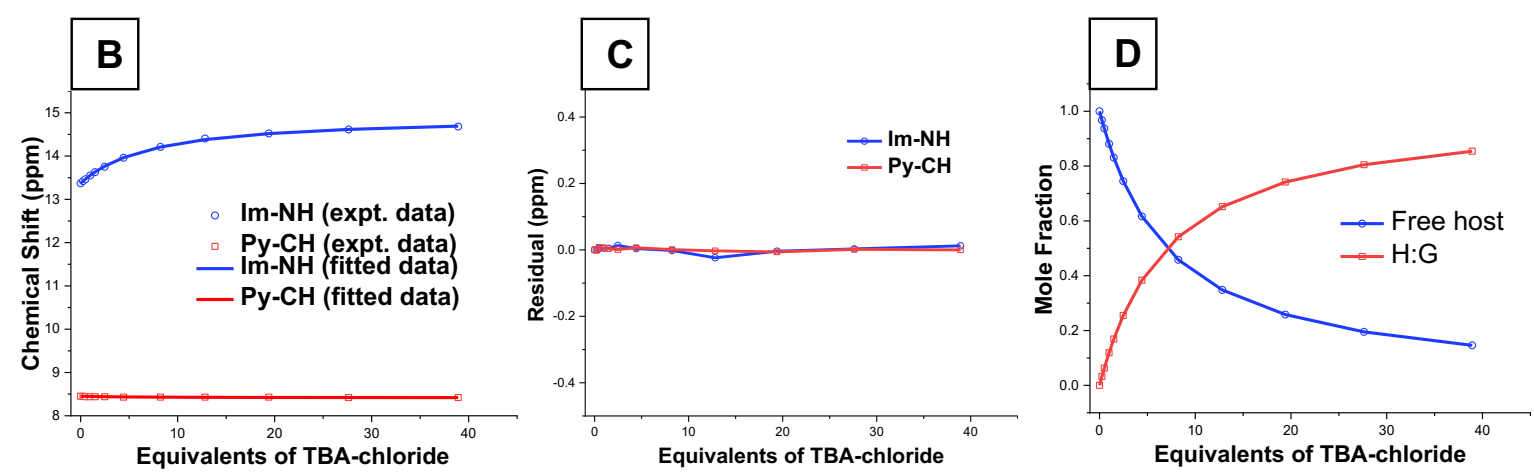

Figure S91: ${ }^{1} \mathrm{H}$ NMR spectroscopic titration of receptor $2(2 \mathrm{mM})$ with TBACl in DMSO-d 6 with $0.5 \%$ water at $298 \mathrm{~K}$. (a) Stack plot. (b) Fitplot for $\mathrm{NH}$ proton at $\delta=13.37 \mathrm{ppm}$ (proton1, Im$\mathrm{NH}$ ) and $\mathrm{CH}$ proton at $\delta=8.45 \mathrm{ppm}$ (proton2, $\mathrm{Py}-\mathrm{CH}$ ) using global analysis with 1:1 host:guest stoichiometry $\left(\mathrm{K}_{\mathrm{a}}=77 \mathrm{M}^{-1}\right.$, error: $\left.2 \%\right)$. (c) Plot of the residuals for $\delta=13.37 \mathrm{ppm}$ (proton1) and $\delta$ $=8.45 \mathrm{ppm}$ (proton2) using global analysis. (d) Calculated mole fractions. 

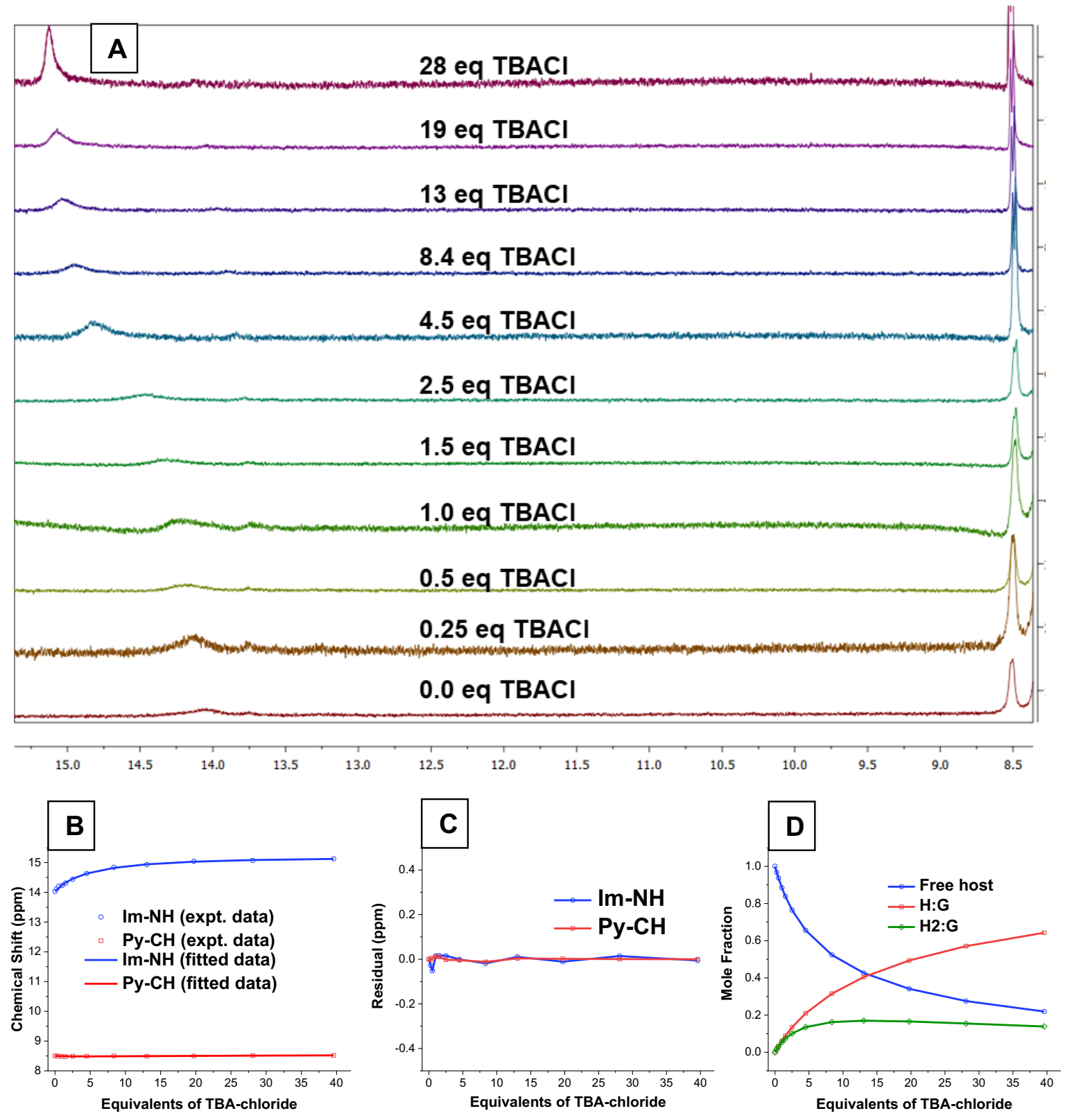

Figure S92: ${ }^{1} \mathrm{H}$ NMR spectroscopic titration of receptor $3(2 \mathrm{mM})$ with TBACl in DMSO$d_{6}$ with $\mathbf{0 . 5 \%}$ water at $298 \mathrm{~K}$. (a) Stack plot. (b) Fitplot for $\mathrm{NH}$ proton at $\delta=14.03 \mathrm{ppm}$ (proton1, Im- $\mathrm{NH}$ ) and $\mathrm{CH}$ proton at $\delta=8.50 \mathrm{ppm}$ (proton2, $\mathrm{Py}-\mathrm{CH}$ ), using global analysis with $1: 1\left(\mathrm{~K}_{\mathrm{a}}=133 \mathrm{M}^{-1}\right.$, error: $6 \%$ ). (c) Plot of the residuals for $\delta=14.03 \mathrm{ppm}$ (proton1) and $\delta=$ $8.50 \mathrm{ppm}$ (proton2) using global analysis. (d) Calculated mole fractions. 

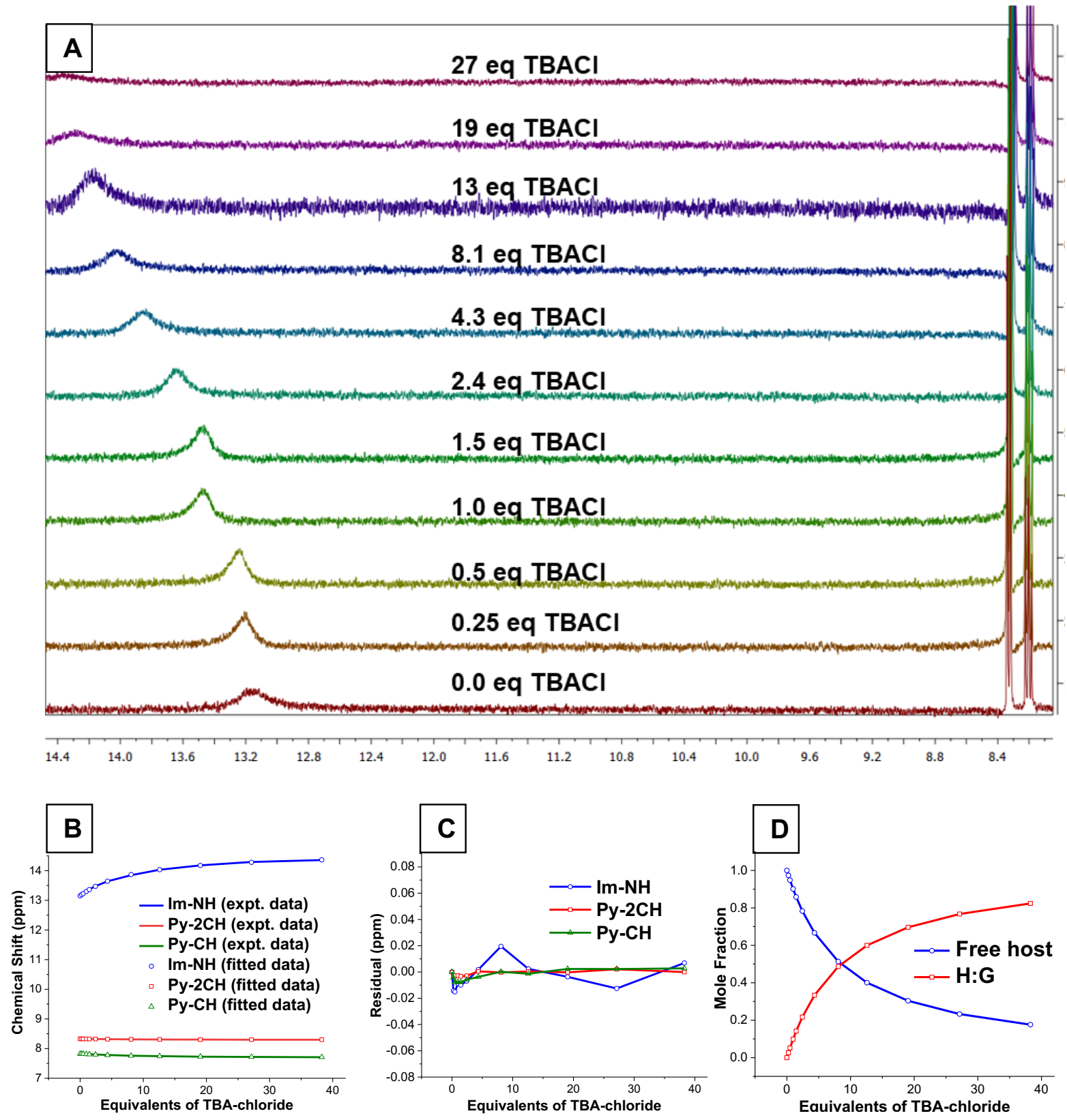

Figure S93: ${ }^{1} \mathrm{H}$ NMR spectroscopic titration of receptor $1(2 \mathrm{mM})$ with TBACl in DMSO- $d_{6}$ with $0.5 \%$ water at $298 \mathrm{~K}$. (a) Stack plot. (b) Fitplot for $\mathrm{NH}$ proton at $\delta=13.15 \mathrm{ppm}$ (proton1, Im-NH) and $\mathrm{CH}$ protons at $\delta=8.33 \mathrm{ppm}$ (proton2, $\mathrm{Py}-2 \mathrm{CH}$ ) and $\delta=7.83 \mathrm{ppm}$ (proton3, $\mathrm{Py}-\mathrm{CH}$ ), using global analysis with 1:1 host:guest stoichiometry $\left(K_{a}=63 \mathrm{M}^{-1}\right.$, error: $\left.2 \%\right)$. (c) Plot of the residuals for $\delta=13.15 \mathrm{ppm}$ (proton1), $\delta=8.33 \mathrm{ppm}$ (proton2) and $\delta=7.83 \mathrm{ppm}$ (proton3) using global analysis. (d) Calculated mole fractions. 


\section{S8: Reduction kinetic studies:}

Three reducing agents were used in the current study, namely: the tripetide glutathione (GSH), dithiothreitol (DTT) and $\mathrm{HCl}$ of tris(2-carboxyethyl)phosphine (TCEP) (Figure S65). The reduction kinetic study of complexes 6-9 has been done in organic solvent (DMSO), in liposomes with external addition of the reducing agent and in GSH encapsulated liposomes and was monitored by UV-Vis and fluorescence spectroscopies (Figure S65). Absorbance studies UV-Vis spectra were recorded on an Agilent Cary 100 UV-Vis spectrophotometer, equipped with a temperature-controlled multicell holder and a temperature control unit. Fluorescence spectra were conducted on an Agilent Cary Eclipse fluorescence spectrophotometer, equipped with a temperature control unit and a magnetic stirrer. In the current study GSH was used in 6:1, relative to tested compound, while DTT and TCEP were used in 3:1, relative to tested compound.
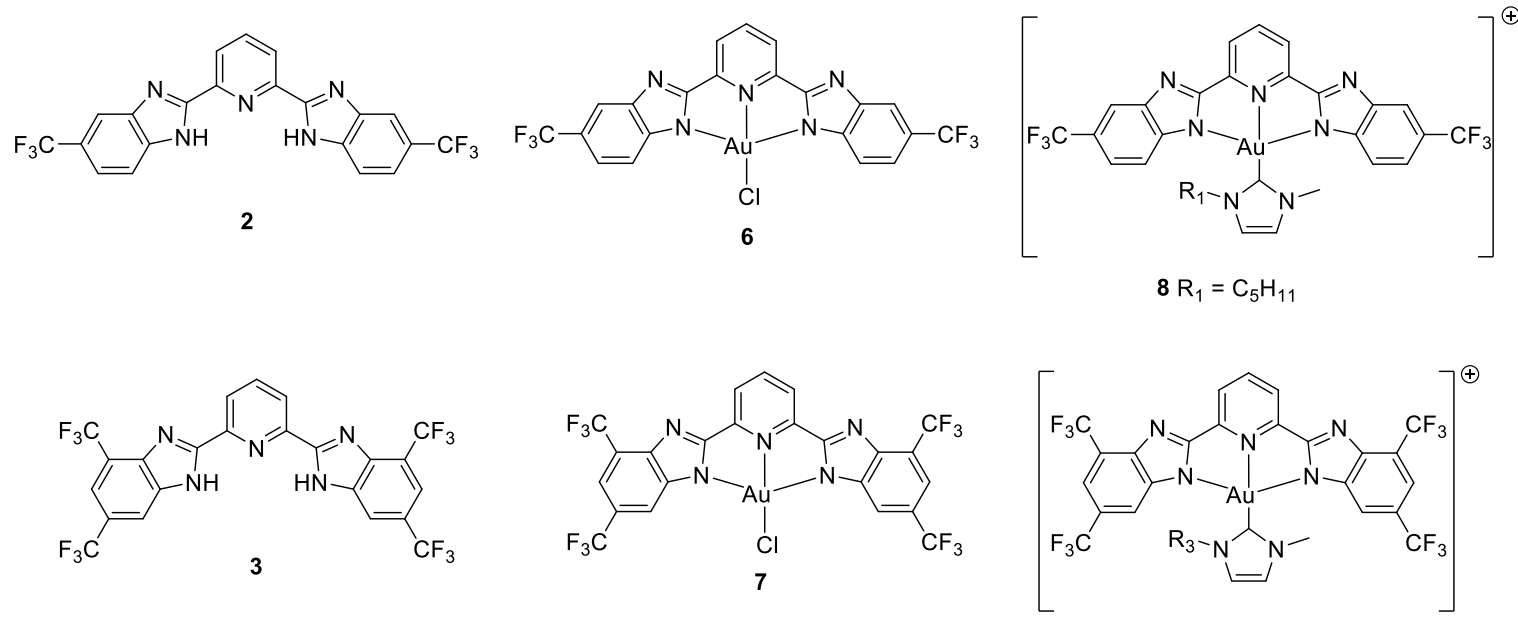

$9 \mathrm{R}_{1}=\mathrm{C}_{5} \mathrm{H}_{11}$

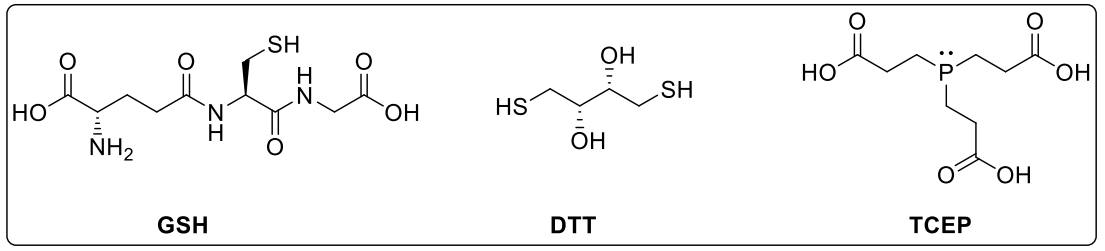

Figure S94: Chemical structure of tested compounds and the three reducing agents used in the current study. 
Reactivity with different thiols was quantified by measuring the increase in fluorescence at $365 \mathrm{~nm}$ (285 nm excitation) for probe 2 and complexes 6 and 8 and at $400 \mathrm{~nm}$ (350 $\mathrm{nm}$ excitation) for probe 3 and complexes 7 and 9 over time and the increase in absorbance at $325 \mathrm{~nm}$ for all tested compounds over time. Receptors 2 and $\mathbf{3}$ and complexes 6-9 were dissolved in DMSO at room temperature to afford the probe concentration at $0.5 \mathrm{mM}$. GSH, DTT and TCEP stock solutions (3.0 mM, $1.5 \mathrm{mM}, 1.5 \mathrm{mM}$, respectively) WERE freshly prepared in deionized water for the DMSO experiments and in the external solution for the experiments conducted in liposomes. Generally, $5 \mu \mathrm{L}(0.5$ $\mathrm{mM}$, final concentation $=1 \mu \mathrm{M}$ ) of tested compounds were used in fluorescenc experiments, while low absorbance was detected for this concentration in UV-Vis, the loading volume of tested compounds was changed to $25 \mu \mathrm{L}(0.5 \mathrm{mM}$, final concentation $=5 \mu \mathrm{M}$ ). The absorbance or the emission spectra at a certain wavelength (noted for each compound above) was plotted against the wavelength using OriginPro 9.1.

\section{S7.1: Reduction kinetics in DMSO:}

UV-Vis: In a quartz cuvette and at $25^{\circ} \mathrm{C}$, tested compound $(25 \mu \mathrm{L}, 0.5 \mathrm{mM})$ was added to DMSO $2450 \mu \mathrm{L}$. The reducing agent $(25 \mu \mathrm{L}) \mathrm{GSH}(3.0 \mathrm{mM})$, DTT $(3.0 \mathrm{mM})$ or TCEP $(3.0 \mathrm{mM})$ was added with stirred for 30 seconds. A baseline absorbance was measured on $2475 \mu \mathrm{L}$ DMSO and $25 \mu \mathrm{L}$ of the reducing agent, and spectra were collected at 1 minute intervals over a period of 15 minutes. After 15 minutes, there was no significant change in the absorbance intensity. Results are the average of at least two repeats.

Fluorescence: DMSO $(2490 \mu \mathrm{L})$ and tested compound $(5 \mu \mathrm{L})$ were added to a standard disposable cuvette with stirring and at $25^{\circ} \mathrm{C}$. Reducing agent $(5 \mu \mathrm{L}) \mathrm{GSH}(3.0 \mathrm{mM})$, DTT (3.0 mM) or TCEP (3.0 mM) was added and stirred for 30 seconds and spectra was collected every minute for 30 minutes. Results are the average of at least three repeats.

\section{S7.2: Reduction kinetics in liposomes:}

Unilamellar $\mathrm{KCl}(100 \mathrm{mM})$ vesicles, buffered with $10 \mathrm{mM} \mathrm{HEPES}$ at $\mathrm{pH}$ 7.0, were prepared as mentioned earlier in section S6.1, however, without addition of HPTS probes. The and UV-Vis and fluorescence properties of receptors $\mathbf{2}$ and $\mathbf{3}$ were exploited to monitor the 
reduction kinetics of complexes 6-9. It was noted that both transporters 2 and 3 reatined their UV-Vis properties and had the same $\lambda_{\max }$ as in organic solvent experiment (DMSO, S7.1). Transporter 2 retained its fluorescence nature in the $\mathrm{KCl}$-liposomes, while transporter 3 fluoresecne properties diminshed, when it was added to liposomes. Thus reduction kinetics of complexes 6 and 7 was monitored by UV-Vis and fluoresence, while reduction complexes 8 and 9 to the free anion transporter 3 could be only monitored by UV-Vis.

UV-Vis: In a quartz cuvette and at $25^{\circ} \mathrm{C}$, tested compound ( $\left.25 \mu \mathrm{L}, 0.5 \mathrm{mM}\right)$ was added to $2450 \mu \mathrm{L}$ of the liposomal suspension. The reducing agent $(25 \mu \mathrm{L}) \mathrm{GSH}(3.0 \mathrm{mM})$, DTT (3.0 mM) or TCEP $(3.0 \mathrm{mM})$ was added with stirred for 30 seconds. A baseline absorbance was measured on $2450 \mu \mathrm{L}$ liposomal suspension $25 \mu \mathrm{L}$ of reducing agent, and $25 \mu \mathrm{L}$ DMSO. Spectra were collected at 5 minutes intervals over a period of 3-4 $\mathrm{h}$. Results are the average of at least two repeats.

Fluorescence: Fluorescence spectra were recorded on excitation wavelength $285 \mathrm{~nm}$ and emission wavelengths $300-500\left(\lambda_{\mathrm{ex}}=285 \mathrm{~nm}, \lambda_{\mathrm{em}}=300-500 \mathrm{~nm}\right)$. Liposomal suspension $(2490 \mu \mathrm{L})$ and tested compound $(5 \mu \mathrm{L})$ were added to a standard disposable cuvette with stirring and at $25^{\circ} \mathrm{C}$. Reducing agent $(5 \mu \mathrm{L}) \mathrm{GSH}(3.0 \mathrm{mM})$, DTT $(3.0 \mathrm{mM})$ or TCEP (3.0 $\mathrm{mM}$ ) was added and stirred for 30 seconds and spectra was collected at 1 minutes and for 1.5-3.0 h. Results are the average of at least three repeats.

\section{S7.3: Reduction kinetics in encapsolated GSH Liposomes:}

Assuming the fraction of the interior volume of $200 \mathrm{~nm}$ unilammelar vesicles was $0.059 \%$, two different $10 \mathrm{mM}$ (final concentration in $2.5 \mathrm{~mL}=6 \mu \mathrm{M}$ ) and $50 \mathrm{mM}$ (final concentration in $2.5 \mathrm{~mL}=30 \mu \mathrm{M}) \mathrm{GSH}$ encapsulated $\mathrm{KCl}$ liposomes, buffered with $10 \mathrm{mM}$ HEPES at $\mathrm{pH} 7.0$, were made. A chloroform solution of POPC $(\sim 30 \mathrm{mg} / \mathrm{mL})$ was evaporated under vacuum and dried for at least $6 \mathrm{~h}$. The thin film was hydrated by the internal solution containing GSH (10 mM or $50 \mathrm{mM}$ ) and was was subjected to nine freeze-thaw cycles 
followed by extrusion 25 times through a $200 \mathrm{~nm}$ polycarbonate membrane. Size exclusion chromatography using sephadex G-25 column and HPTS-free external solution as an eluent was conducted to remove unentrapped GSH from the vesicles' solution. The internal and external solutions used were $\mathrm{KCl}(100 \mathrm{mM})$ buffered with $10 \mathrm{mM}$ HEPES at $\mathrm{pH}$ 7.0. Finally, for each measurement, external solution $(\mathrm{KCl})$ was used to dilute the lipid tock to obtain $2.5 \mathrm{~mL}$ lipid suspension containing $0.1 \mathrm{mM}$ lipid. A base pulse of $\mathrm{KOH}$ ( 25 $\mu \mathrm{L}, 0.5 \mathrm{M}$ ) at final concentration $5 \mathrm{mM}$ was added to generate a transmembrane $\mathrm{pH}$ gradient. After the tested receptors were added, compound 2 fluorescence spectra of excitation $=285 \mathrm{~nm}$ and emission $=300-500$ were recorded. Compound 3 and its complexes 7 and 9 were excluded as receptor 3 fluorescence properties diminished fluorescence in liposomal solution.

UV-Vis and fluorescence reduction kinetic experiments were used as in section S7.2, where $50 \mathrm{mM} \mathrm{GSH}$ encapsulated vesicles used in UV-Vis study and $10 \mathrm{mM} \mathrm{GSH}$ encapsulated vesicles used in fluorescence studies, correseponding to 1:6 compound:GSH in each case. 


\section{- Reduction kinetics of complex 6 with GSH}

FigureS95: Monitoring of reduction of complex 6 by GSH in A- DMSO and in B- Liposomes using UV-Vis and fluorescence spectroscopies. Fluorescence and UV-Vis readings are averages of at least two replicates, always with standard deviations less than $10 \%$.

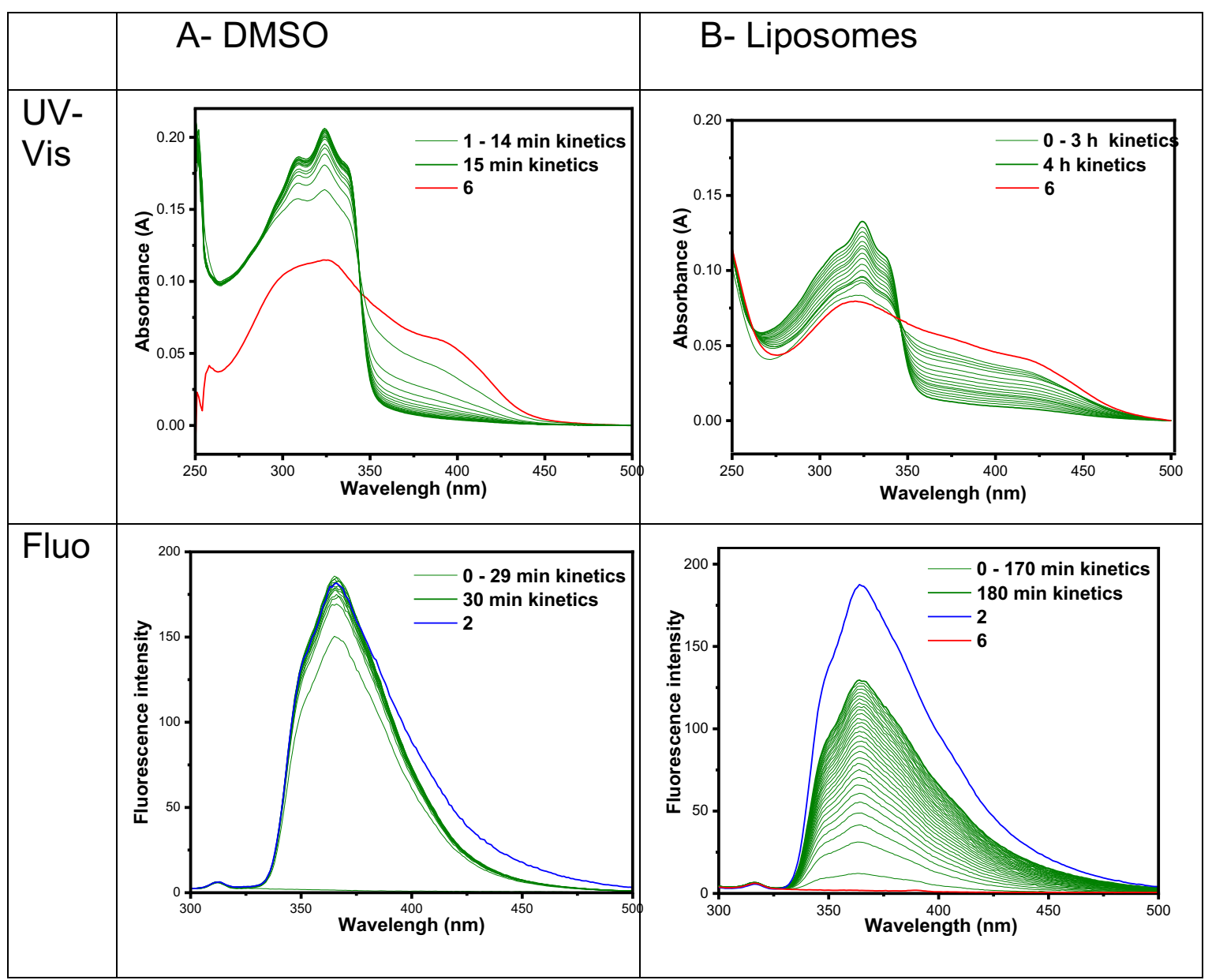




\section{- Reduction kinetics of complex 7 with GSH}

FigureS96: Monitoring of reduction of complex 7 by GSH in A- DMSO and in B- Liposomes using UV-Vis and fluorescence spectroscopies. Fluorescence and UV-Vis readings are averages of at least two replicates, always with standard deviations less than $10 \%$.

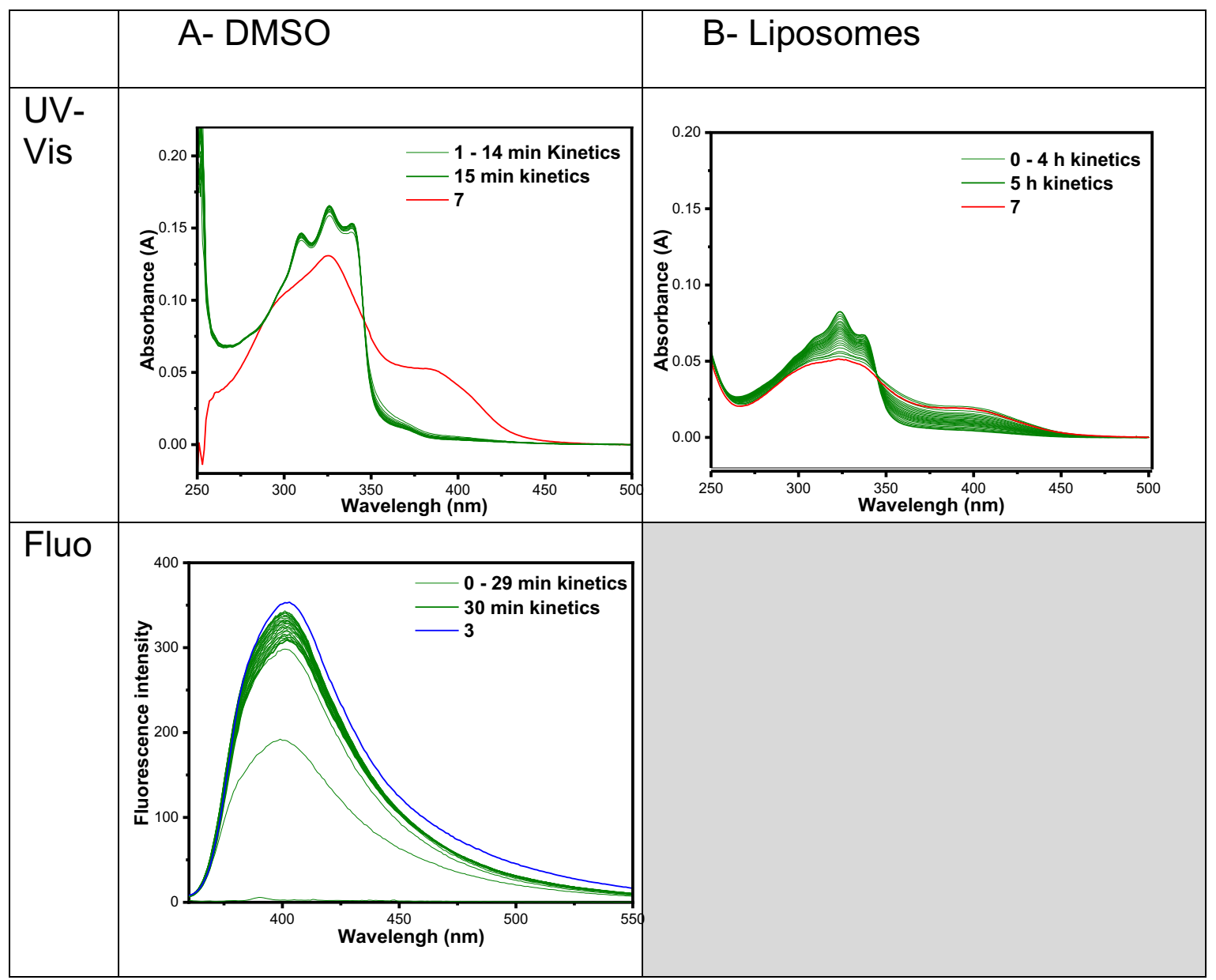




\section{- Reduction kinetics of complex 8 with GSH}

FigureS97: Monitoring of reduction of complex 8 by GSH in A- DMSO and in B- Liposomes using UV-Vis and fluorescence spectroscopies. Fluorescence and UV-Vis readings are averages of at least two replicates, always with standard deviations less than $10 \%$.

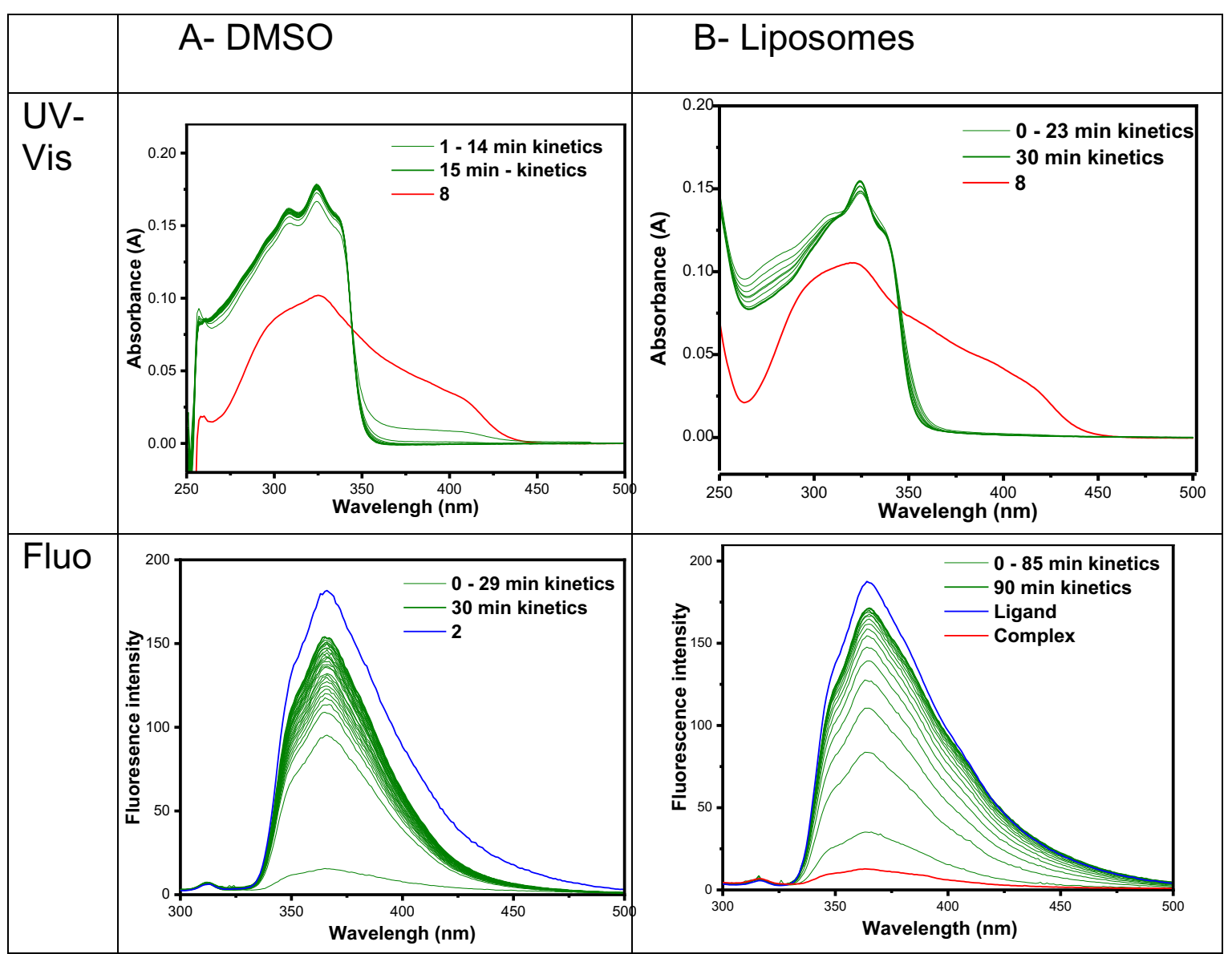




\section{- Reduction kinetics of complex 9 with GSH}

FigureS98: Monitoring of reduction of complex 9 by GSH in A- DMSO and in B- Liposomes using UV-Vis and fluorescence spectroscopies. Fluorescence and UV-Vis readings are averages of at least two replicates, always with standard deviations less than $10 \%$.

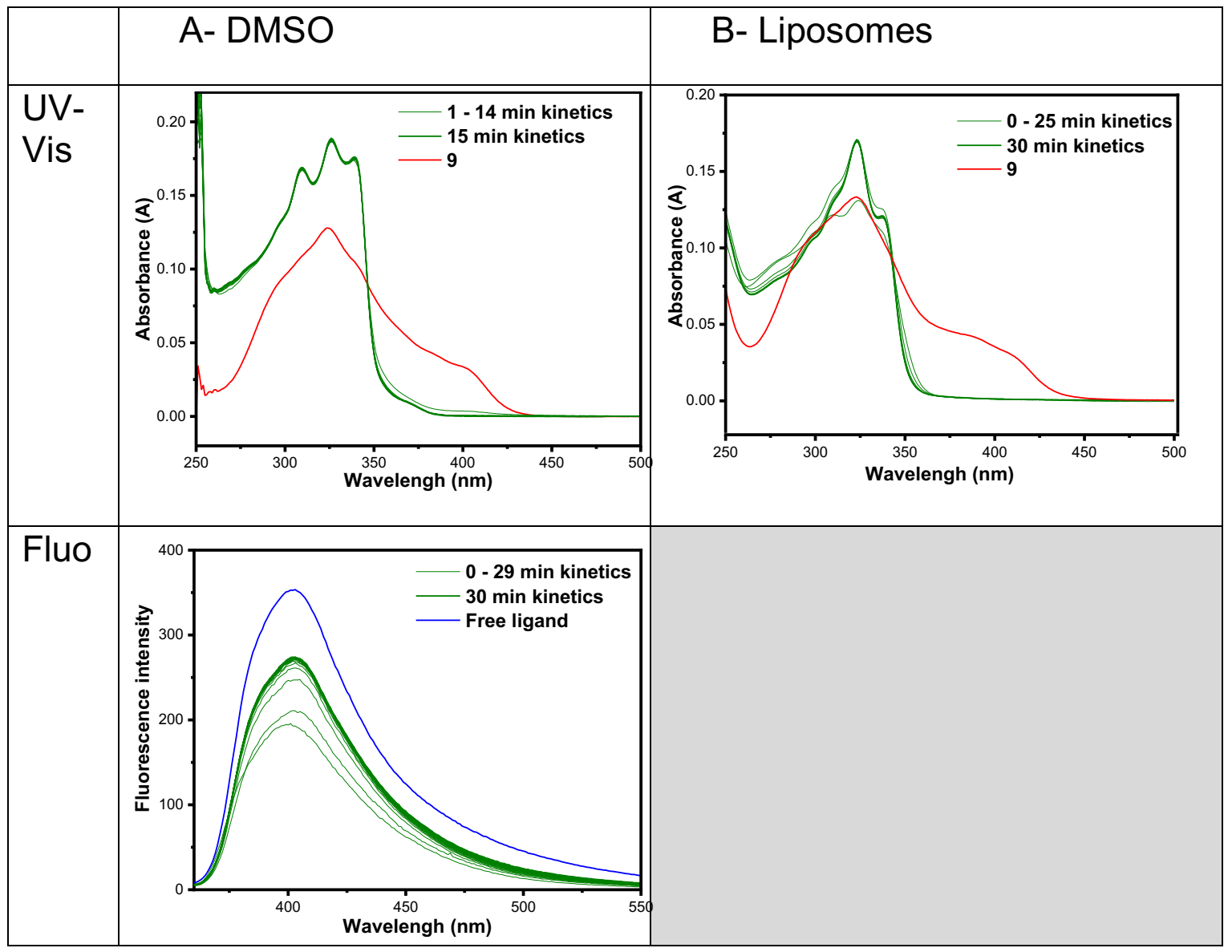


- Reduction kinetics of complex 6 with GSH in GSH encapsulated liposomes

FigureS99: Monitoring of reduction of complex 6 by GSH encapsulated Liposomes using A- UV$V$ is and b- fluorescence spectroscopies. Fluorescence and UV-Vis readings are averages of at least two replicates, always with standard deviations less than $10 \%$.

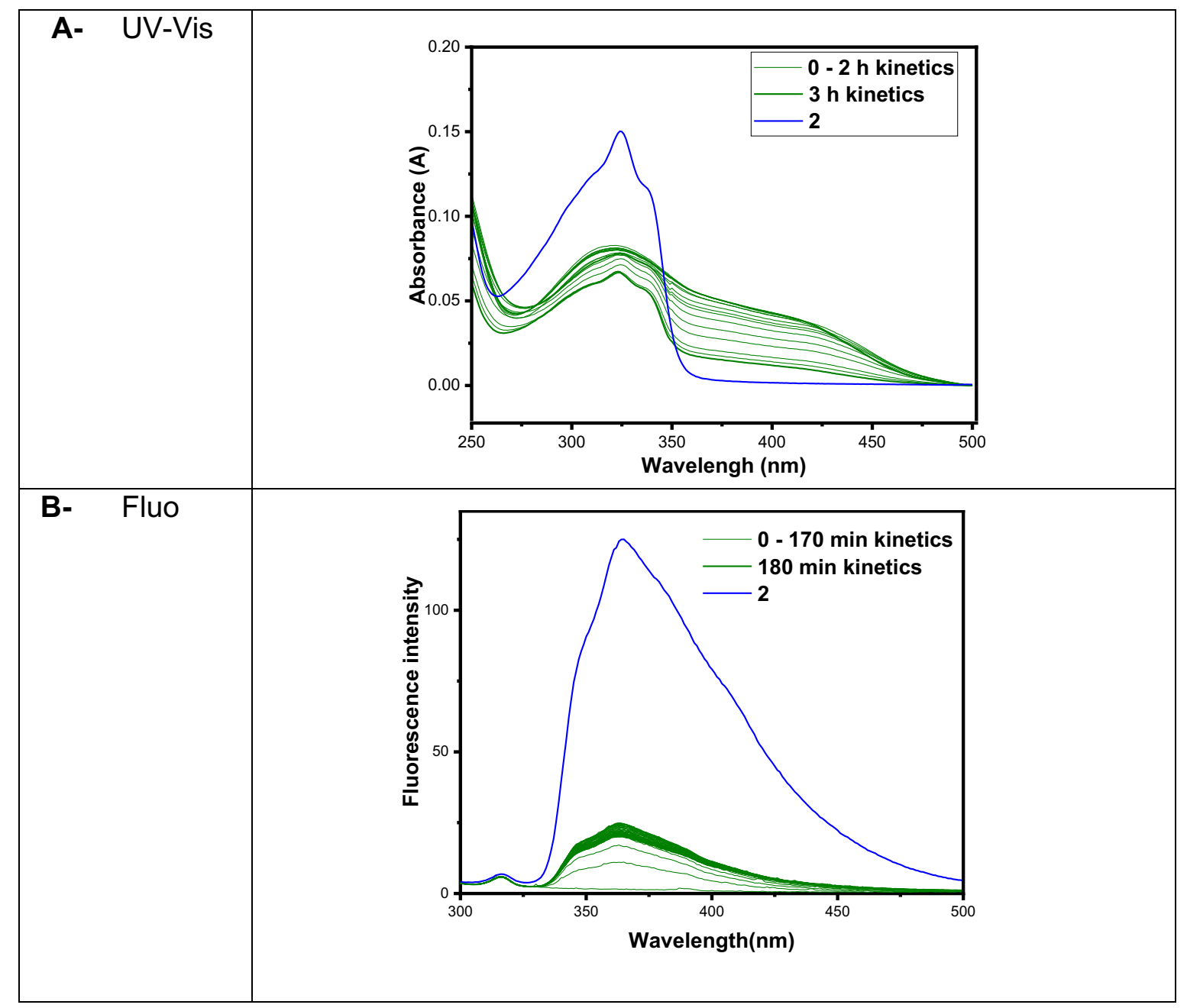


- Reduction kinetics of complex 7 with GSH in GSH encapsulated liposomes

FigureS100: Monitoring of reduction of complex 7 by GSH encapsulated Liposomes using UVVic spectroscopy. UV-Vis readings are averages of at least two replicates, always with standard deviations less than $10 \%$.

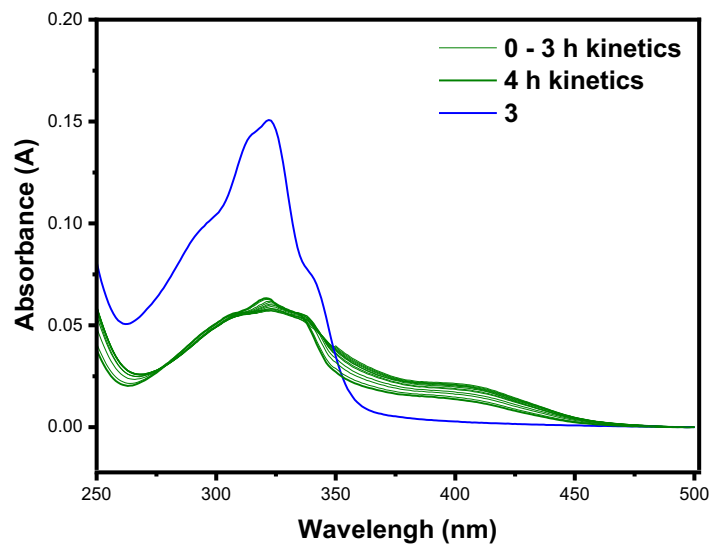

S76 
- Reduction kinetics of complex 8 with GSH in GSH encapsulated liposomes

FigureS101: Monitoring of reduction of complex 8 by GSH encapsulated Liposomes using A- UV$V$ is and b- fluorescence spectroscopies. Fluorescence and UV-Vis readings are averages of at least two replicates, always with standard deviations less than $10 \%$.

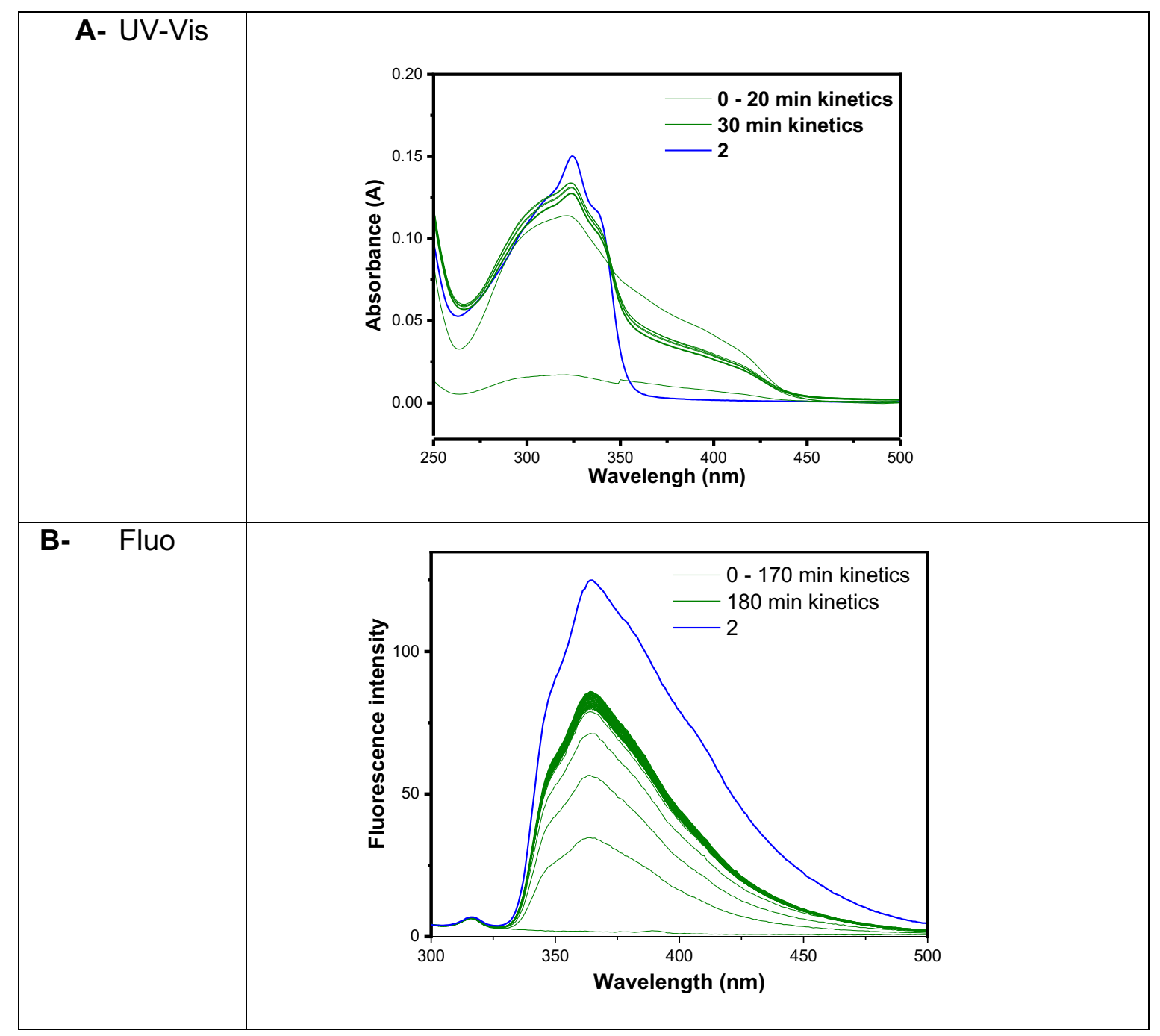


- Reduction kinetics of complex 9 with GSH in GSH encapsulated liposomes

FigureS102: Monitoring of reduction of complex 9 by GSH encapsulated Liposomes using UVVis spectroscopy. UV-Vis readings are averages of at least two replicates, always with standard deviations less than $10 \%$.

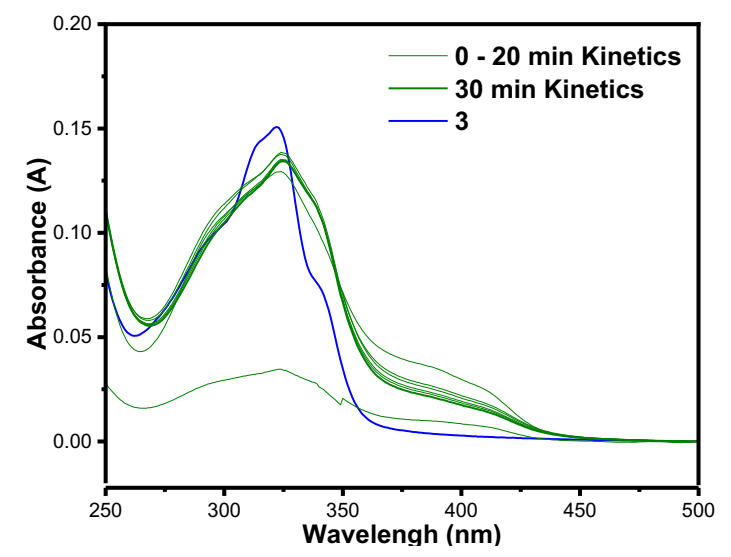


- Reduction kinetics of complex 6 with GSH (Fluorescence):

FigureS103: Comparison of complex 6 reduction by different modes of GSH addition in Liposomes using fluorescence spectroscopy. Fluorescence readings are averages of at least three replicates, always with standard deviations less than $5 \%$.

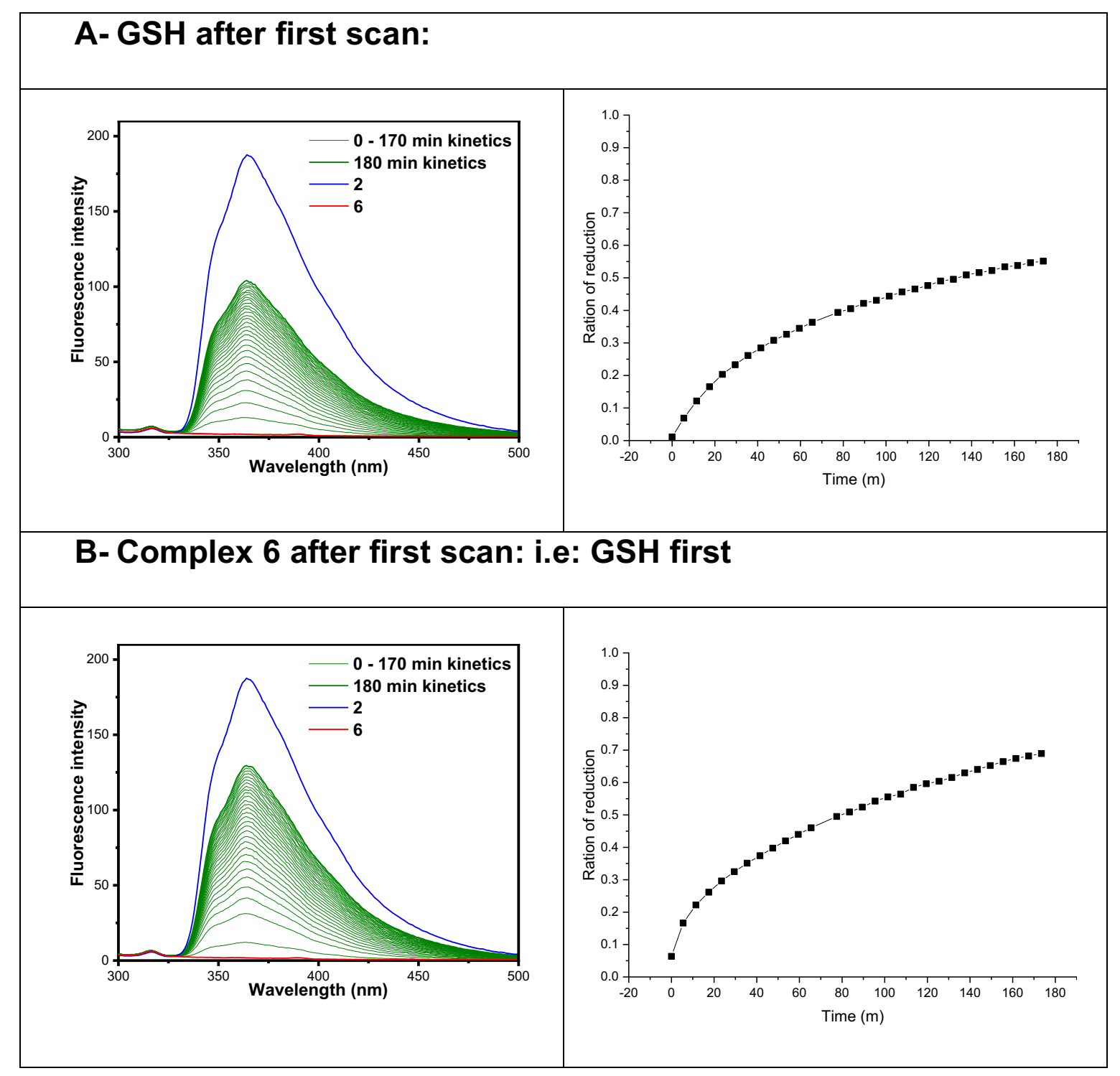




\section{- Reduction kinetics of complex 6 with DTT}

FigureS104: Monitoring of reduction of complex 6 by DTT in A- DMSO and in B- Liposomes using UV-Vis and fluorescence spectroscopies. Fluorescence and UV-Vis readings are averages of at least two replicates, always with standard deviations less than $10 \%$.

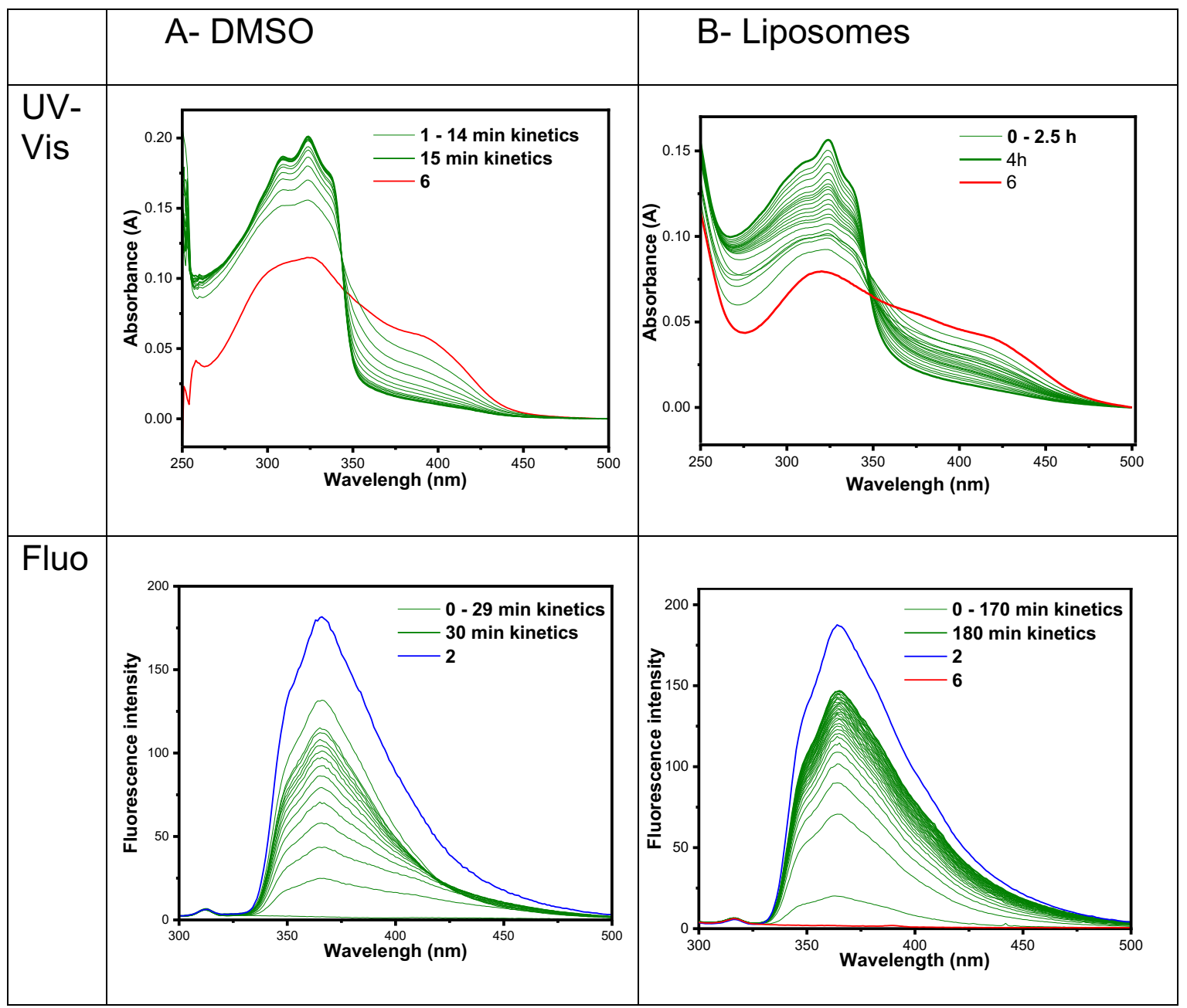




\section{- Reduction kinetics of complex 7 with DTT}

FigureS105: Monitoring of reduction of complex 7 by DTT in A- DMSO and in B- Liposomes using UV-Vis and fluorescence spectroscopies. Fluorescence and UV-Vis readings are averages of at least two replicates, always with standard deviations less than $10 \%$.

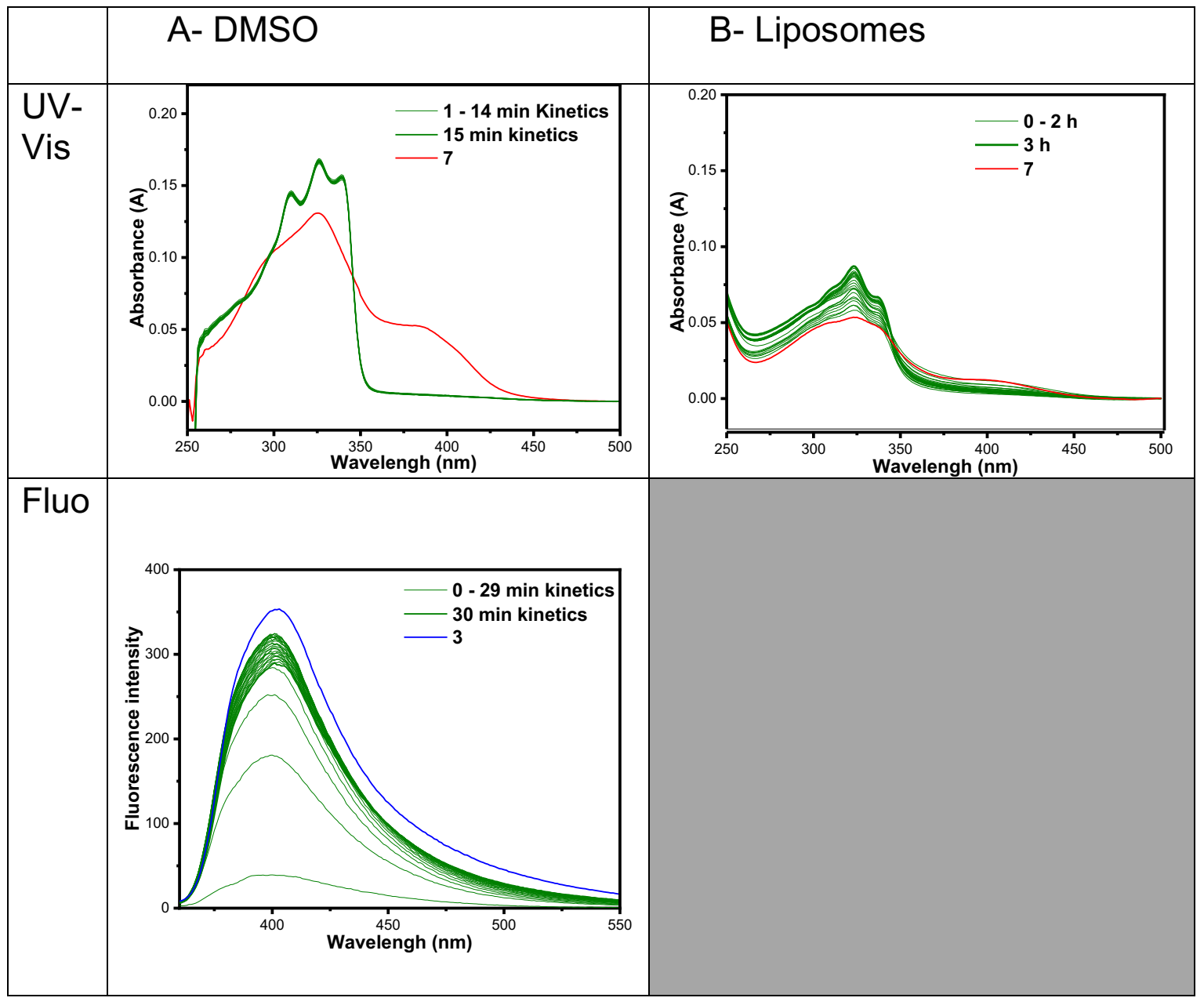




\section{- Reduction kinetics of complex 8 with DTT}

Figure S106: Monitoring of reduction of complex 8 by DTT in A- DMSO and in B- Liposomes using UV-Vis and fluorescence spectroscopies. Fluorescence and UV-Vis readings are averages of at least two replicates, always with standard deviations less than $10 \%$.

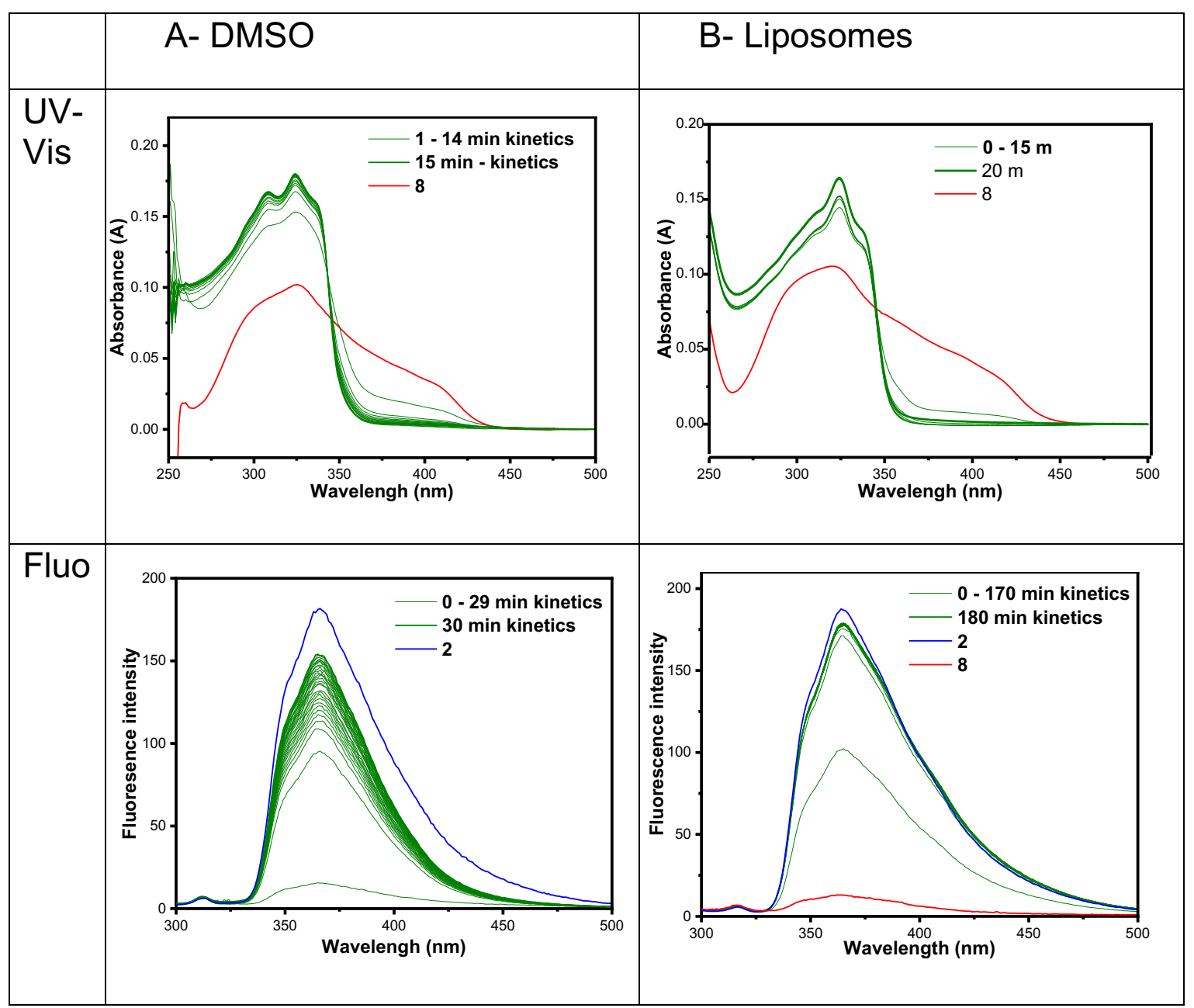




\section{- Reduction kinetics of complex 9 with DTT}

FigureS107: Monitoring of reduction of complex 9 by DTT in A- DMSO and in B- Liposomes using UV-Vis and fluorescence spectroscopies. Fluorescence and UV-Vis readings are averages of at least two replicates, always with standard deviations less than $10 \%$.

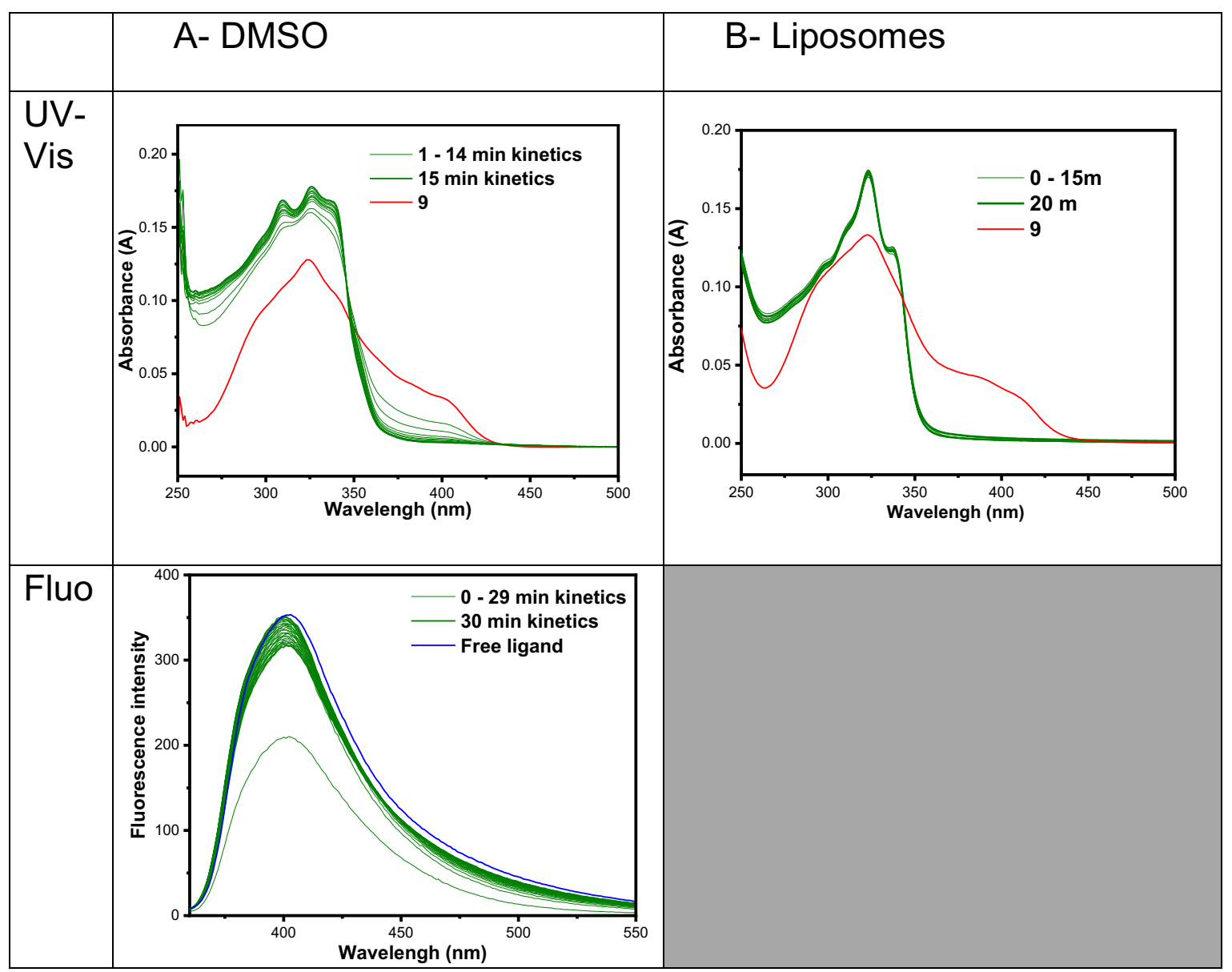




\section{- Reduction kinetics of complex 6 with TCEP}

FigureS108: Monitoring of reduction of complex 6 by TCEP in A- DMSO and in B- Liposomes using UV-Vis and fluorescence spectroscopies. Fluorescence and UV-Vis readings are averages of at least two replicates, always with standard deviations less than $10 \%$.

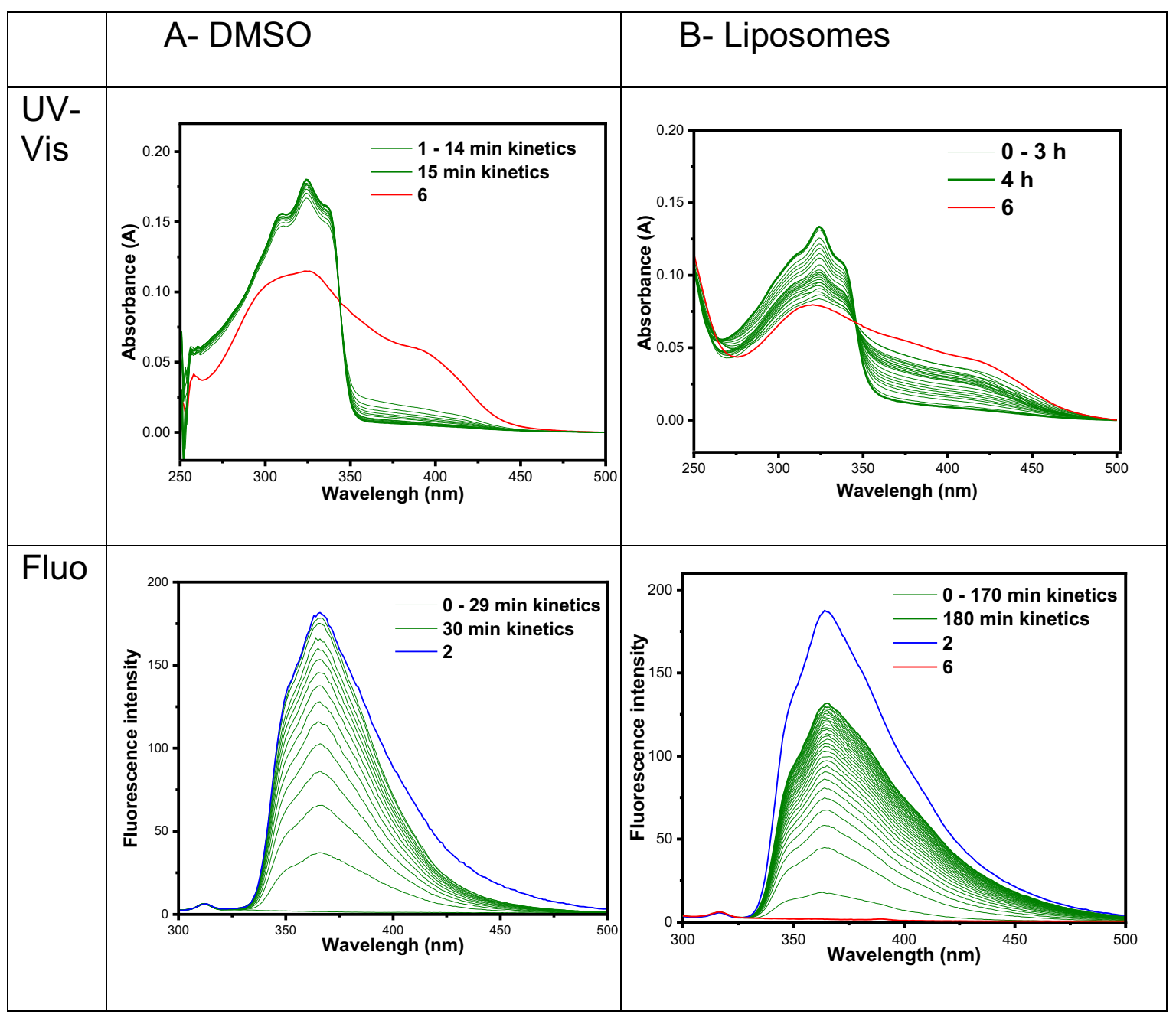




\section{- Reduction kinetics of complex 7 with TCEP}

FigureS109: Monitoring of reduction of complex 7 by TCEP in A- DMSO and in B- Liposomes using UV-Vis and fluorescence spectroscopies. Fluorescence and UV-Vis readings are averages of at least two replicates, always with standard deviations less than $10 \%$.

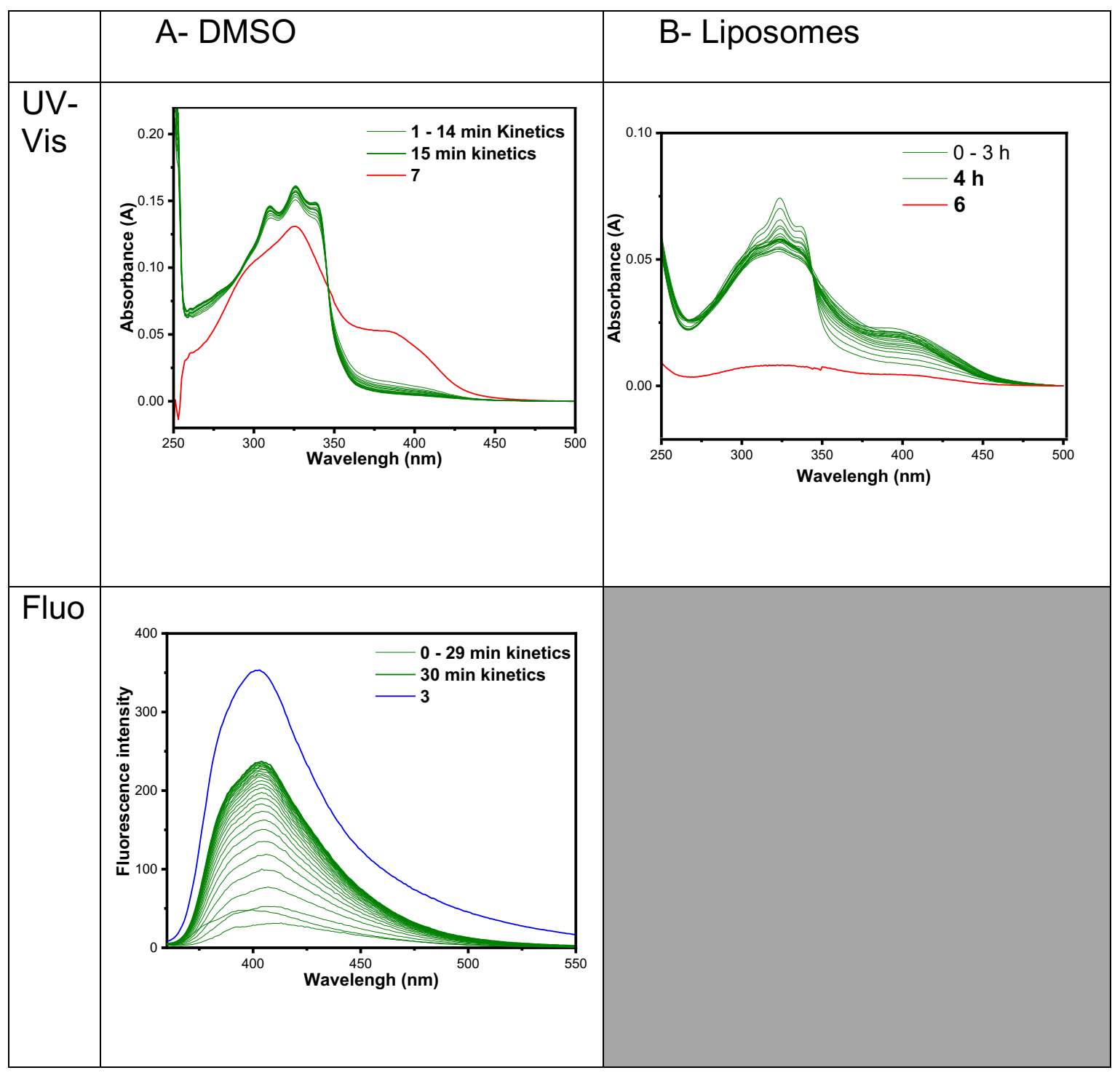




\section{- Reduction kinetics of complex 8 with TCEP}

FigureS110: Monitoring of reduction of complex 8 by TCEP in A- DMSO and in B- Liposomes using UV-Vis and fluorescence spectroscopies. Fluorescence and UV-Vis readings are averages of at least two replicates, always with standard deviations less than $10 \%$.

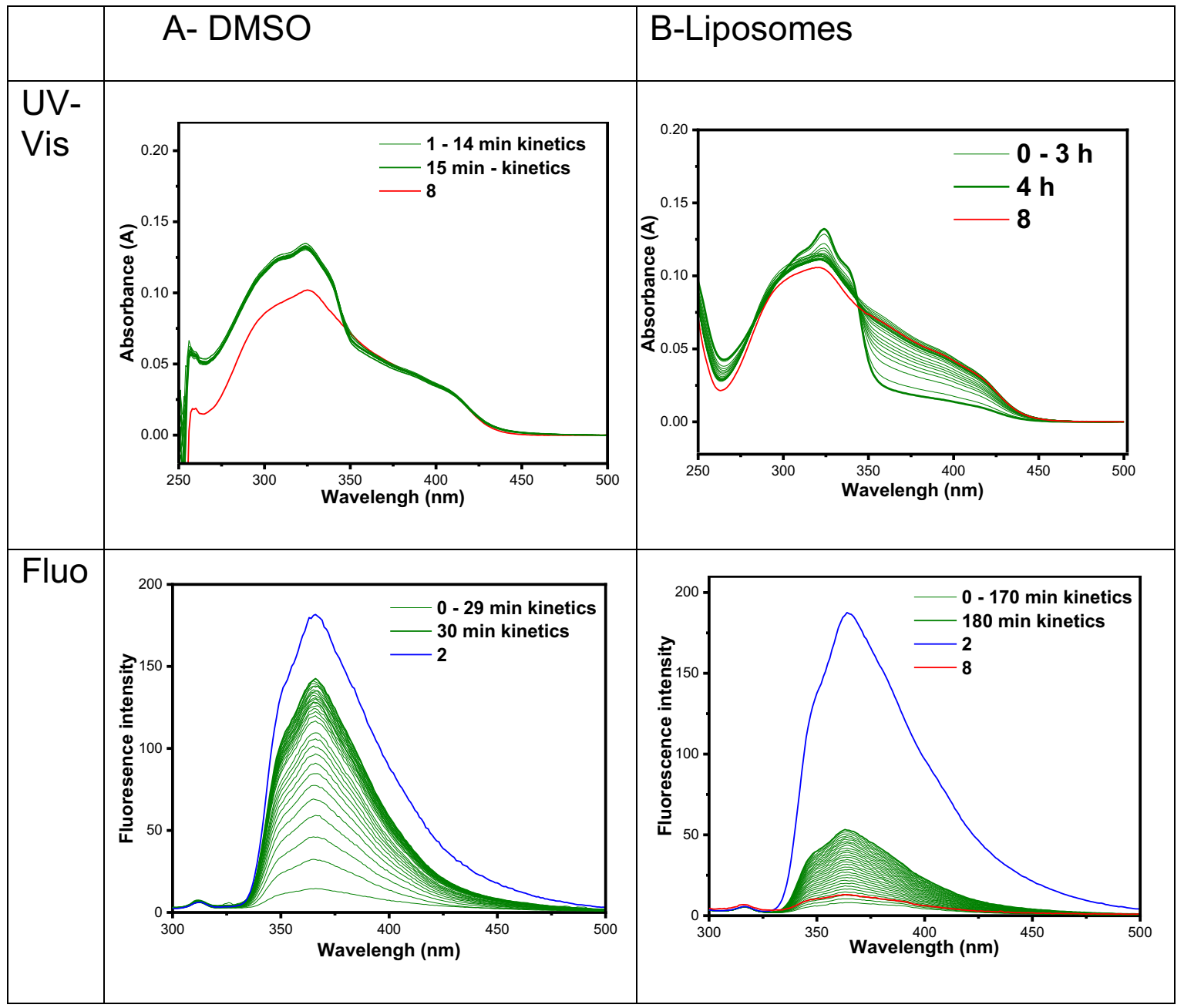




\section{- Reduction kinetics of complex 9 with TCEP}

FigureS111: Monitoring of reduction of complex 9 by TCEP in A- DMSO and in B- Liposomes using UV-Vis and fluorescence spectroscopies. Fluorescence and UV-Vis readings are averages of at least two replicates, always with standard deviations less than $10 \%$.

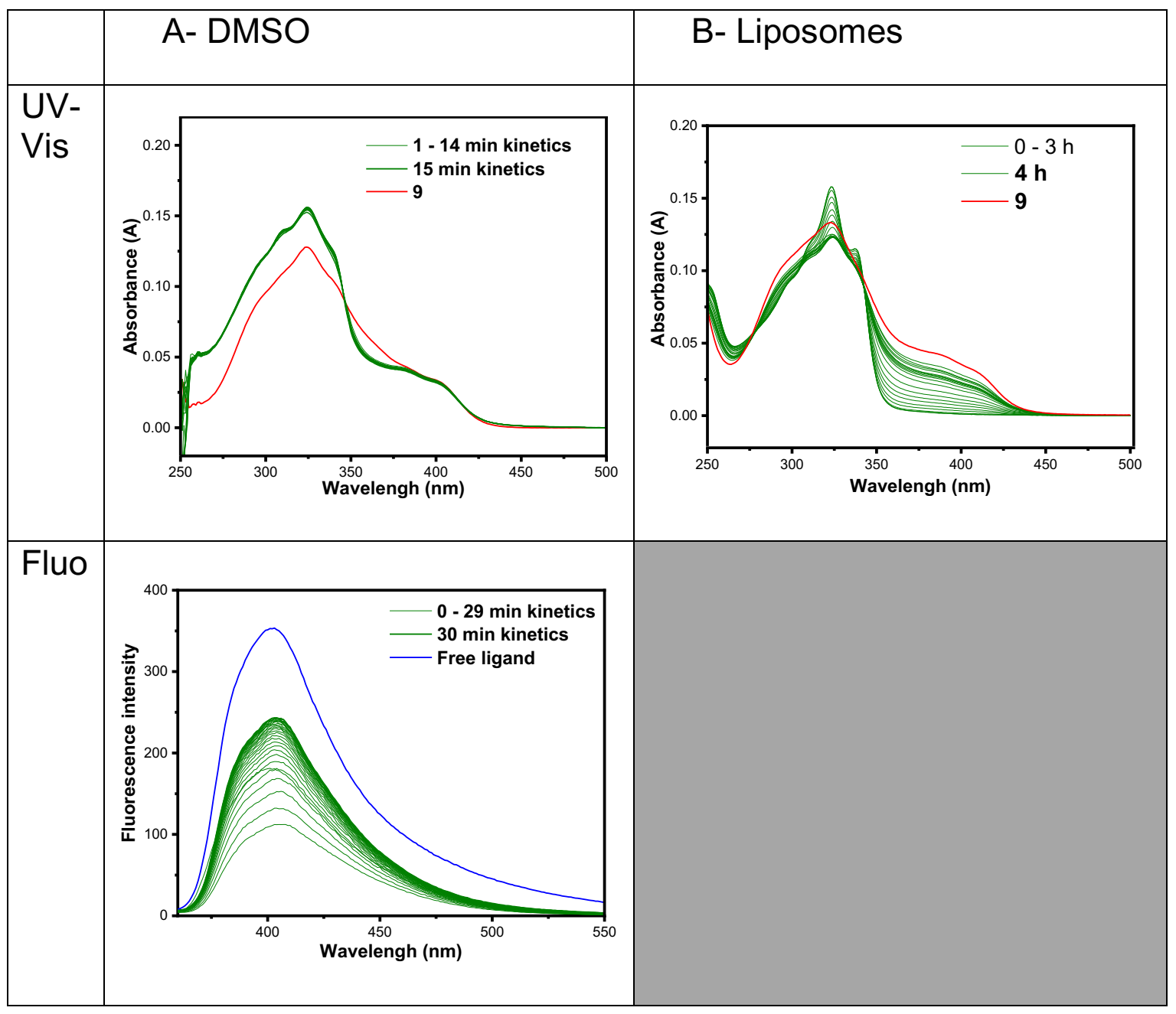


FigureS112: Summary of reduction kinetics of complexes 6-9 by GSH in DMSO, Liposomes and GSH encapsulated liposomes using UV-Vis and fluorescence spectroscopies. Fluorescence and UV-Vis readings are averages of at least two replicates, always with standard deviations less than $10 \%$.

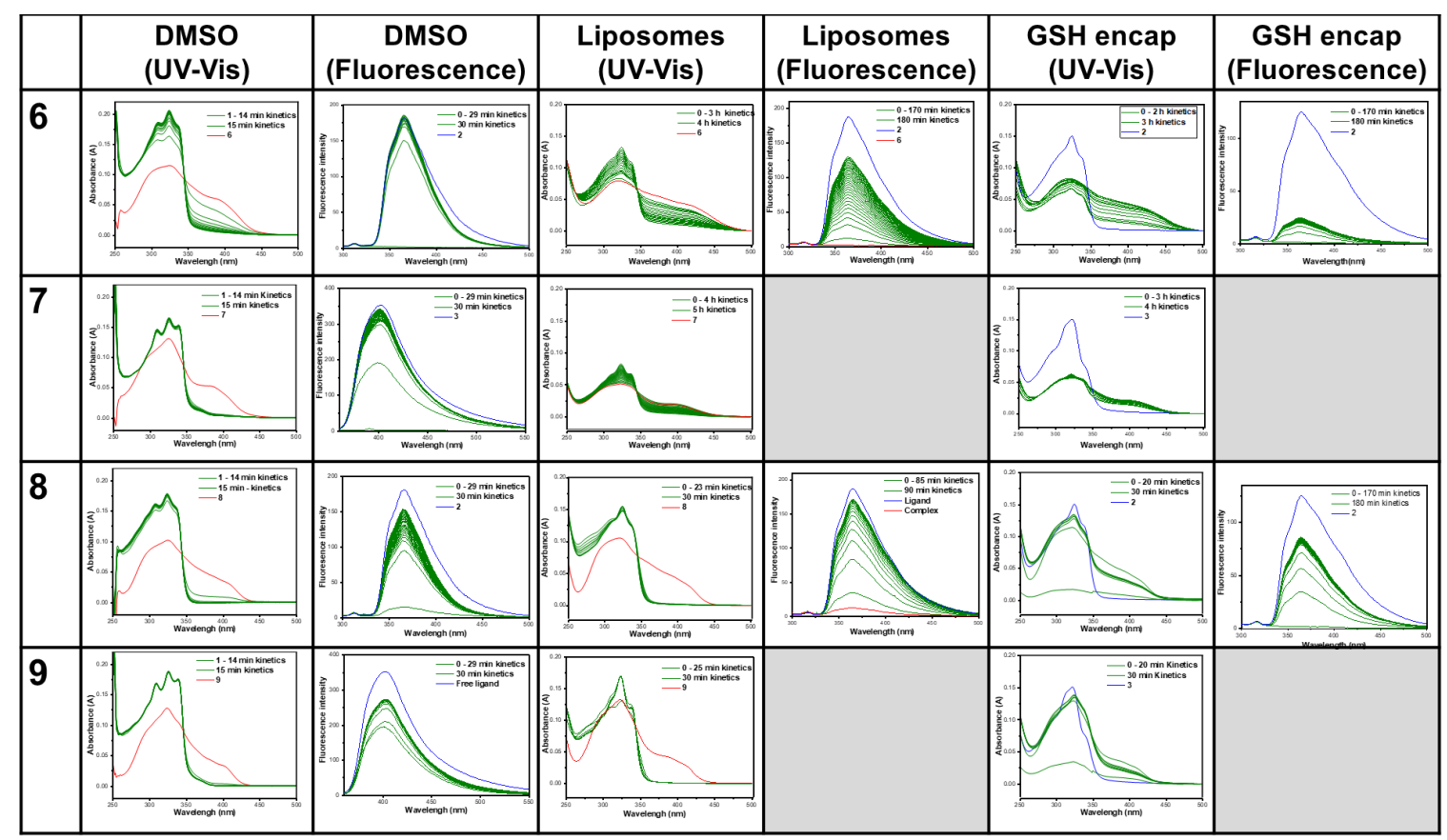




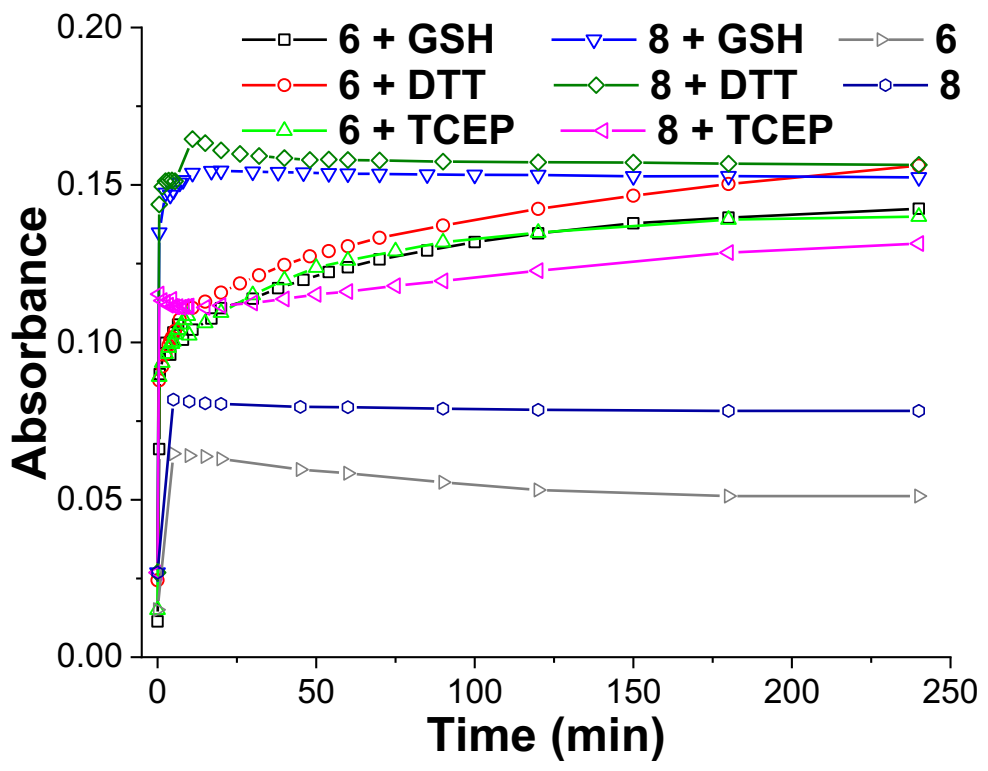

Figure S113: Reduction of complexes 6 and 8 in organic solvent (DMSO) by thiols; namely GSH (reduced gluathione) and DTT (dithiothreitol), and TCEP (tris(2-carboxyethyl)phosphine hydrochloride). Probes were present at $5 \mu \mathrm{M}$, GSH was present at $30 \mu \mathrm{M}$, while DTT and TCEP were kept at $15 \mu \mathrm{M}$ at the final volume. UV-Vis readings are averages of two replicates, always with standard deviations less than $10 \%$. 


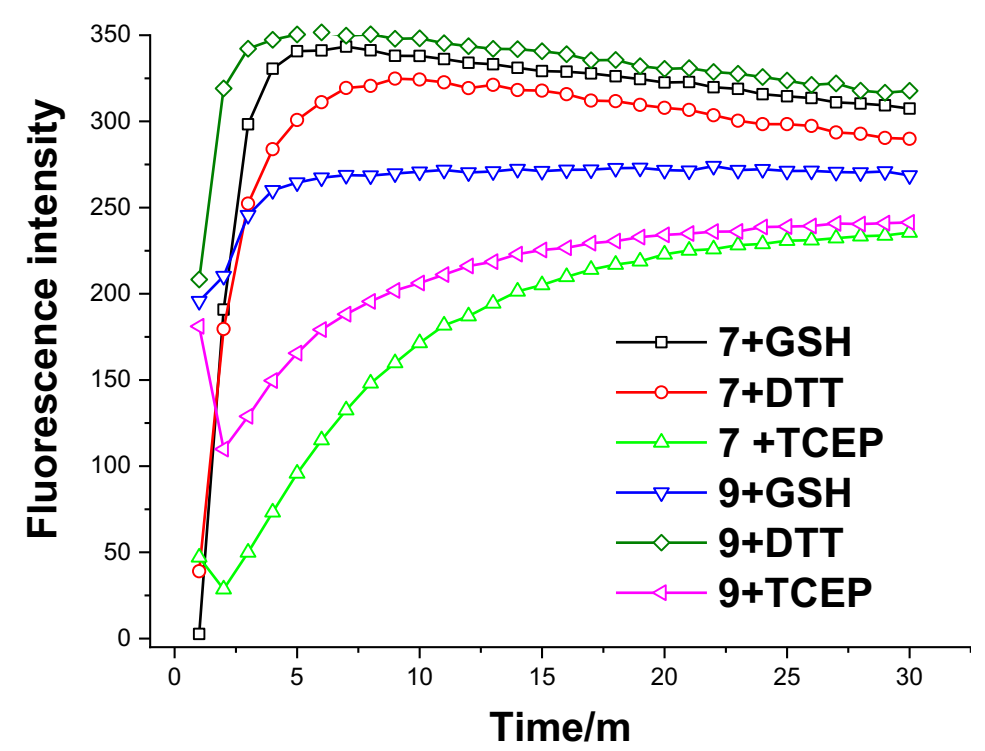

Figure S114: Reduction of complexes 7 and 9 in organic sovent (DMSO) by thiols; namely GSH (reduced gluathione) and DTT (dithiothreitol), and TCEP (tris(2-carboxyethyl)phosphine hydrochloride). Probes were present at $1 \mu \mathrm{M}, \mathrm{GSH}$ was present at $6 \mu \mathrm{M}$, while DTT and TCEP were kept at $3 \mu \mathrm{M}$ at the final volume. Fluorescence readings are averages of three replicates, always with standard deviations less than $10 \%$.

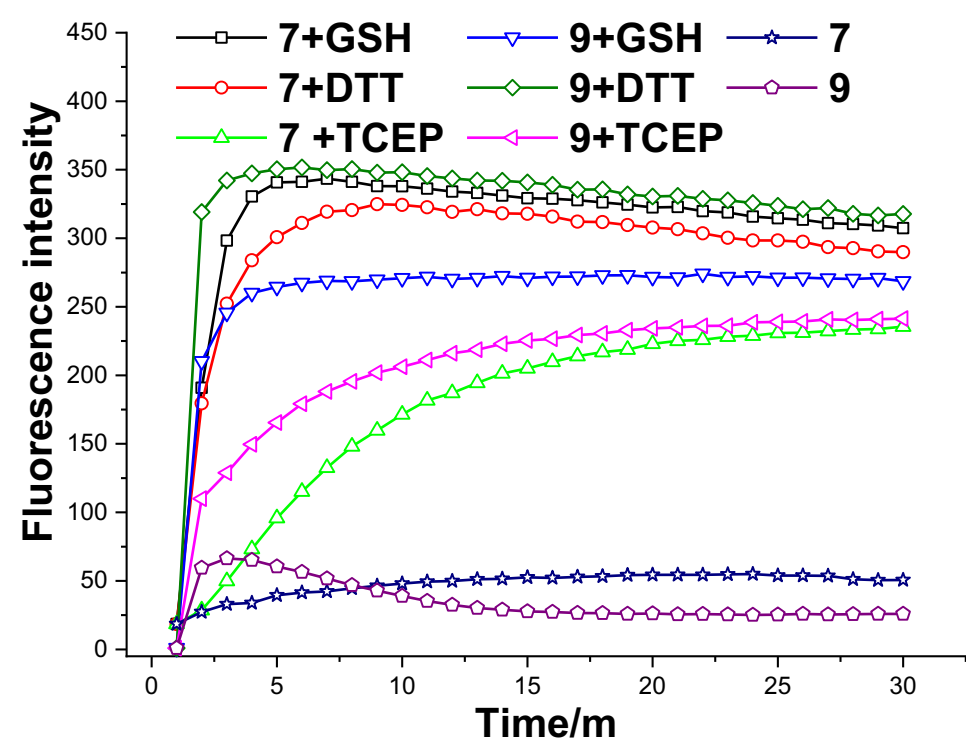

Figure S115: Reduction of complexes 7 and 9 in organic solvent (DMSO) by thiols; namely GSH (reduced gluathione) and DTT (dithiothreitol), and TCEP (tris(2-carboxyethyl)phosphine hydrochloride). Probes were present at $5 \mu \mathrm{M}$, GSH was present at $30 \mu \mathrm{M}$, while DTT and TCEP were kept at $15 \mu \mathrm{M}$ at the final volume. UV-Vis readings are averages of two replicates, always with standard deviations less than $10 \%$. 


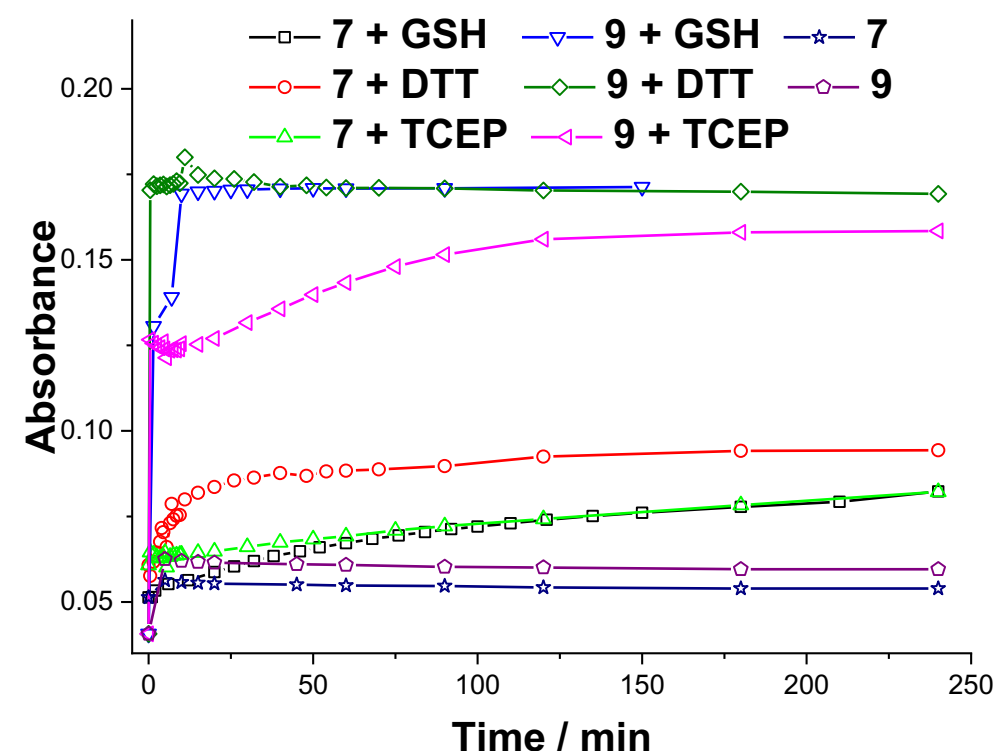

Figure S116: Reduction of complexes 7 and 9 in liposomes by thiols; namely GSH (reduced gluathione) and DTT (dithiothreitol), and TCEP (tris(2-carboxyethyl)phosphine hydrochloride). Probes were present at $5 \mu \mathrm{M}$, GSH was present at $30 \mu \mathrm{M}$, while DTT and TCEP were kept at 15 $\mu \mathrm{M}$ at the final volume. UV-Vis readings are averages of two replicates, always with standard deviations less than $10 \%$. 


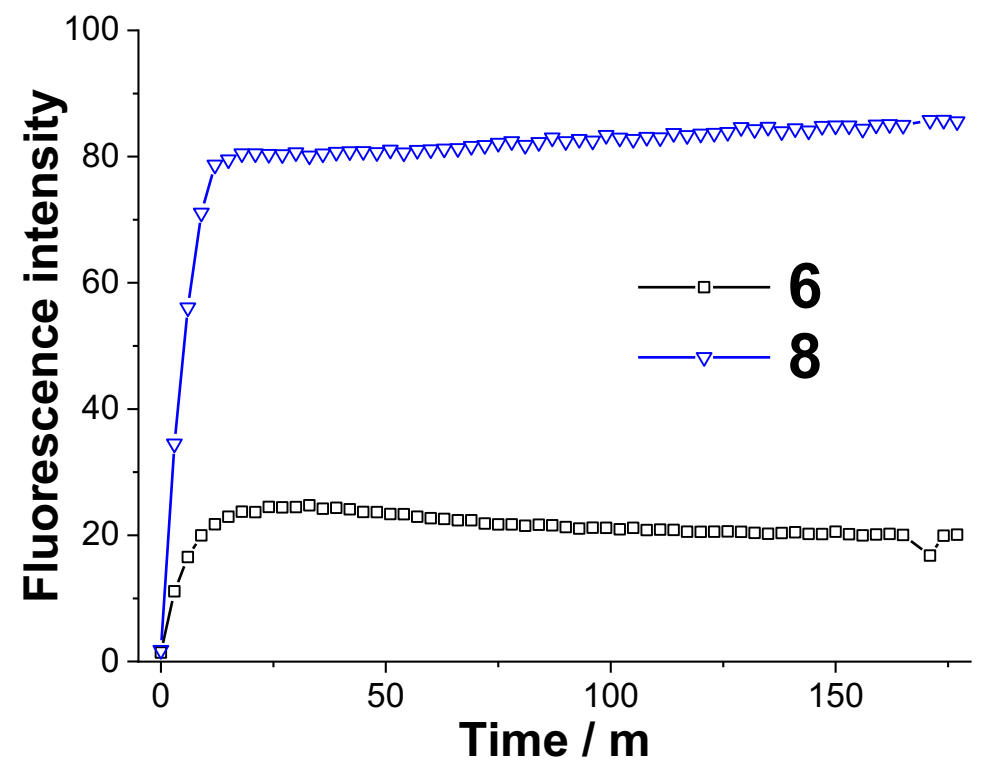

Figure S117: Reduction of complexes 6 and 8 in GSH encapsulated liposomes (10 mM GSH; final GSH concentration $=6 \mu \mathrm{M}$ ). Probes were present at $1 \mathrm{Mm}$ in the final volume. Fluorescence readings are averages of three replicates, always with standard deviations less than $10 \%$.

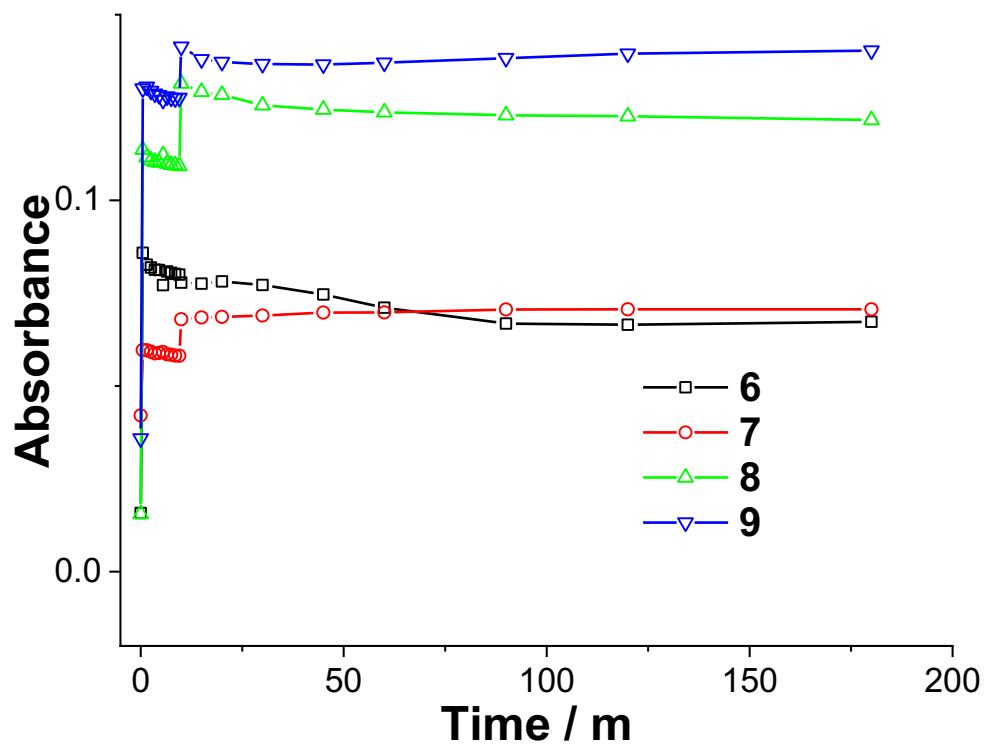

Figure S118: Reduction of complexes 6-8 in GSH encapsulated liposomes (50 mM GSH; final GSH concentration $=30 \mu \mathrm{M}$ ). Probes were present at $5 \mathrm{Mm}$ in the final volume. UV-Vis readings are averages of two replicates, always with standard deviations less than $10 \%$. 


\section{S9: Transport studies upon reduction of complexes 6-9.}

Base-pulse 8-hydroxypyrene-1,3,6-trisulfonic acid trisodium salt (HPTS) assays were conducted using unilamellar 1-palmitoyl-2-oleoylphosphatidylcholine vesicles (POPC) with a mean diameter of $200 \mathrm{~nm}$ loaded with the $\mathrm{pH}$ sensitive fluorescence dye HPTS (1 $\mathrm{mM}) .{ }^{[8]}$ A chloroform solution of POPC ( $\left.30 \mathrm{mg} / \mathrm{mL}\right)$ was evaoprated under vacuum and dried for at least $6 \mathrm{~h}$. The thin film was hydrated by the internal solution containing HPTS $(1 \mathrm{mM})$ and was was subjected to nine freeze-thaw cycles followed by extrusion 25 times through a $200 \mathrm{~nm}$ polycarbonate membrane. Size exclusion chromatography using sephadex G-25 column and HPTS-free external solution as an eluent was conducted to remove unentrapped HPTS from the vesicles' solution. The internal and external solutions used were identical salt solution potassium gluconate (KGlu) or $\mathrm{KCl}$ (100 mM) buffered with $10 \mathrm{mM} \mathrm{HEPES}$ at $\mathrm{pH}$ 7.0. Finally, for each measurement, external solution ( $\mathrm{KCl}$ or $\mathrm{KGlu}$ ) was used to dilute the lipid tock to obtain $2 \mathrm{~mL}$ lipid suspension containing $0.1 \mathrm{mM}$ lipid. A base pulse of $\mathrm{KOH}(25 \mu \mathrm{L}, 0.5 \mathrm{M})$ at a final concentration $5 \mathrm{mM}$ was added to generate a transmembrane $\mathrm{pH}$ gradient. After the tested receptors were added, HPTS fluorescence ratio ( $\lambda_{\mathrm{ex}}=460 \mathrm{~nm}, \lambda_{\mathrm{em}}=510 \mathrm{~nm}$ divided by $\lambda_{\mathrm{ex}}=403 \mathrm{~nm}, \lambda_{\mathrm{em}}=510 \mathrm{~nm}$ ) was recorded. Assisting ionophore (carbonyl cyanide phenylhydrazone (CCCP) or valinomycin) was used as a $5 \mu \mathrm{L}$ DMSO. Bovine serum albumins (BSA) was added to vesicles at $1 \mathrm{~mol} \%$ (with respect to lipid) and allowed to stir for 30 minutes to test if the transport is fatty acid independent, while, oleic acid (1 mol\% and $10 \mathrm{~mol} \%$ ) was used as a source of fatty acid to test if the transport is fatty acid dependent. Detergent $(25 \mu \mathrm{L})$ was added at 200 seconds to destroy the $\mathrm{pH}$ gradient to calibrate the assay. 


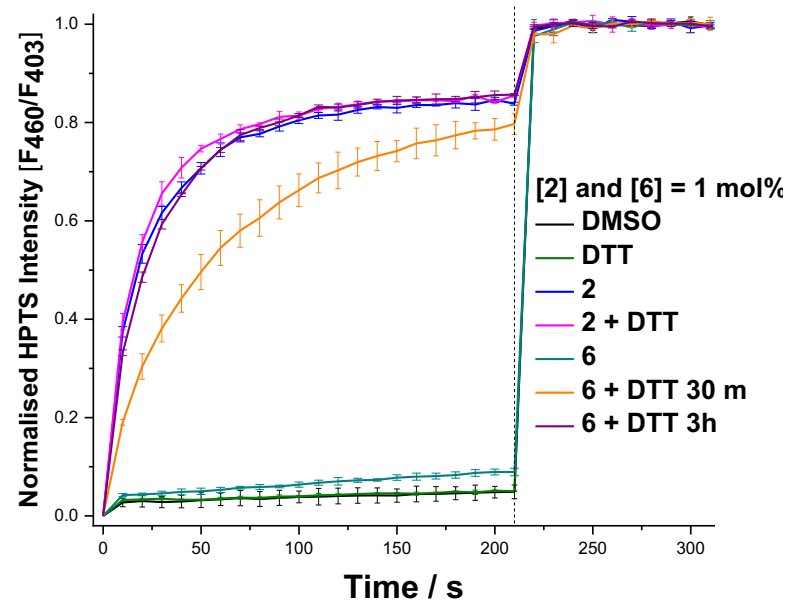

Figure S119: Observed fluorescence ratio response due to $\mathrm{H}^{+} / \mathrm{Cl}^{-}$symport or $\mathrm{Cl}^{-} / \mathrm{OH}^{-}$antiport upon reduction of complex $6(1 \mu \mathrm{M})$ by dithiothreitol DTT $(3 \mu \mathrm{M})$ using $\mathrm{KCl}-\mathrm{KOH}$ assay from POPC vesicles loaded with $\mathrm{KCl}(100 \mathrm{mM})$, buffered to $\mathrm{pH} 7.0$ with HEPES $(10 \mathrm{mM})$ after 30 minutes and 3 hours. The test complex $6(1 \mathrm{~mol} \%)$ and $\mathrm{KOH}$ were added firstly, then DTT was added at $0 \mathrm{~s}$. DMSO, DTT ( $3 \mathrm{~mol} \%$ ), parent anion transporter 2 and complex 6 (without addition of DTT) were used as controls. Detergent was added at $200 \mathrm{~s}$. Ionophore concentrations are shown as ionophore to lipid molar ratios. Error bars represent SD from at least three repeats.

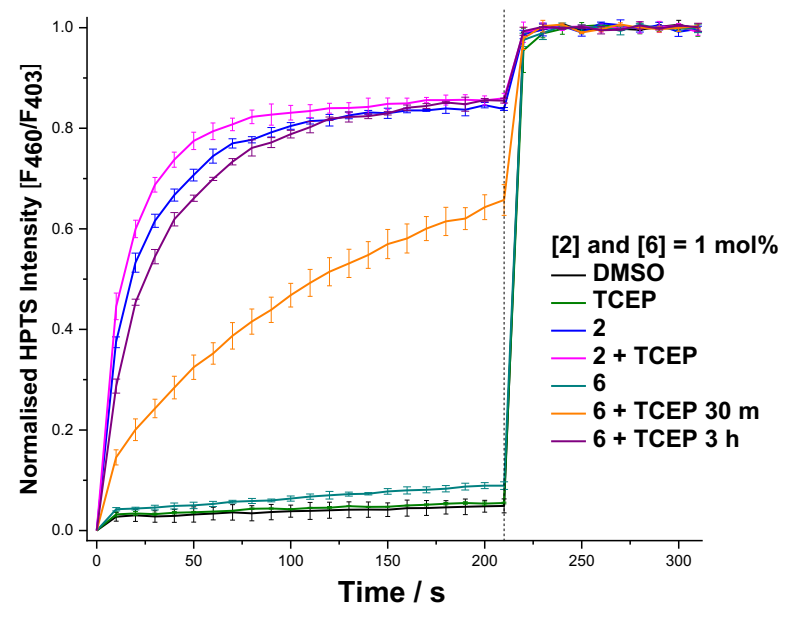

Figure S120: Observed fluorescence ratio response due to $\mathrm{H}^{+} / \mathrm{Cl}^{-}$symport or $\mathrm{Cl}^{-} / \mathrm{OH}^{-}$antiport upon reduction of complex $6(1 \mu \mathrm{M})$ by TCEP $(3 \mathrm{Mm})$ using $\mathrm{KCl}-\mathrm{KOH}$ assay from POPC vesicles loaded with $\mathrm{KCl}(100 \mathrm{mM})$, buffered to $\mathrm{pH} 7.0$ with HEPES $(10 \mathrm{mM})$ after 30 minutes and 3 hours. The test complex $6(1 \mathrm{~mol} \%)$ and $\mathrm{KOH}$ were added firstly, then TCEP was added at $0 \mathrm{~s}$. DMSO, TCEP ( $3 \mathrm{~mol} \%$ ), parent anion transporter $\mathbf{2}$ and complex $\mathbf{6}$ (without addition of TCEP) were used as controls. Detergent was added at $200 \mathrm{~s}$. lonophore concentrations are shown as ionophore to lipid molar ratios. Error bars represent SD from at least three repeats. 


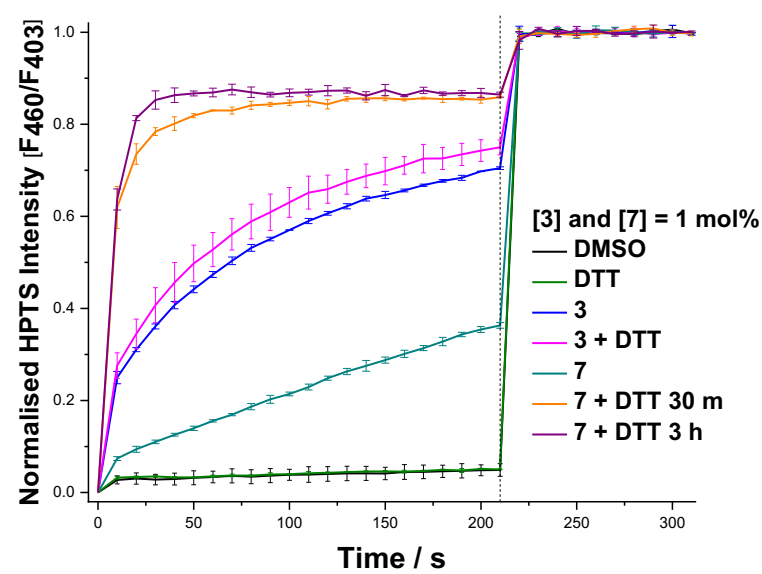

Figure S121: Observed fluorescence ratio response due to $\mathrm{H}^{+} / \mathrm{Cl}^{-}$symport or $\mathrm{Cl}^{-} / \mathrm{OH}^{-}$antiport upon reduction of complex $7(1 \mu \mathrm{M})$ by dithiothreitol DTT $(3 \mathrm{Mm})$ using $\mathrm{KCl}-\mathrm{KOH}$ assay from POPC vesicles loaded with $\mathrm{KCl}(100 \mathrm{mM})$, buffered to $\mathrm{pH} 7.0$ with HEPES (10 mM) after 30 minutes and 3 hours. The test complex $7(1 \mathrm{~mol} \%)$ and $\mathrm{KOH}$ were added firstly, then DTT was added at $0 \mathrm{~s}$. DMSO, DTT ( $3 \mathrm{~mol} \%$ ), parent anion transporter 3 and complex 7 (without addition of DTT) were used as controls. Detergent was added at $200 \mathrm{~s}$. Ionophore concentrations are shown as ionophore to lipid molar ratios. Error bars represent SD from at least three repeats.

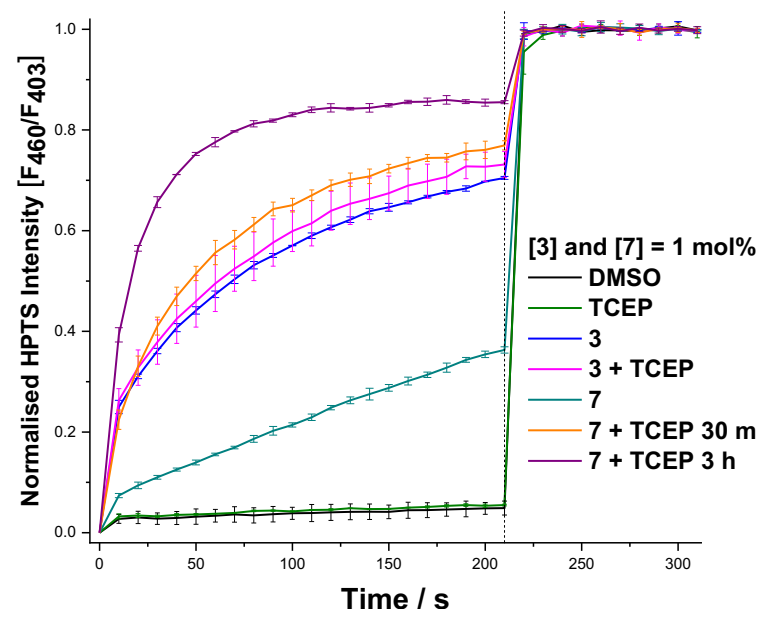

Figure S122: Observed fluorescence ratio response due to $\mathrm{H}^{+} / \mathrm{Cl}^{-}$symport or $\mathrm{Cl}^{-} / \mathrm{OH}^{-}$antiport upon reduction of complex $7(1 \mu \mathrm{M})$ by TCEP $(3 \mathrm{Mm})$ using $\mathrm{KCl}-\mathrm{KOH}$ assay from POPC vesicles loaded with $\mathrm{KCl}(100 \mathrm{mM})$, buffered to $\mathrm{pH} 7.0$ with HEPES $(10 \mathrm{mM})$ after 30 minutes and 3 hours. The test complex 7 ( $1 \mathrm{~mol} \%)$ and $\mathrm{KOH}$ were added firstly, then TCEP was added at $0 \mathrm{~s}$. DMSO, TCEP ( $3 \mathrm{~mol} \%$ ), parent anion transporter 3 and complex 7 (without addition of TCEP) were used as controls. Detergent was added at $200 \mathrm{~s}$. Ionophore concentrations are shown as ionophore to lipid molar ratios. Error bars represent SD from at least three repeats. 


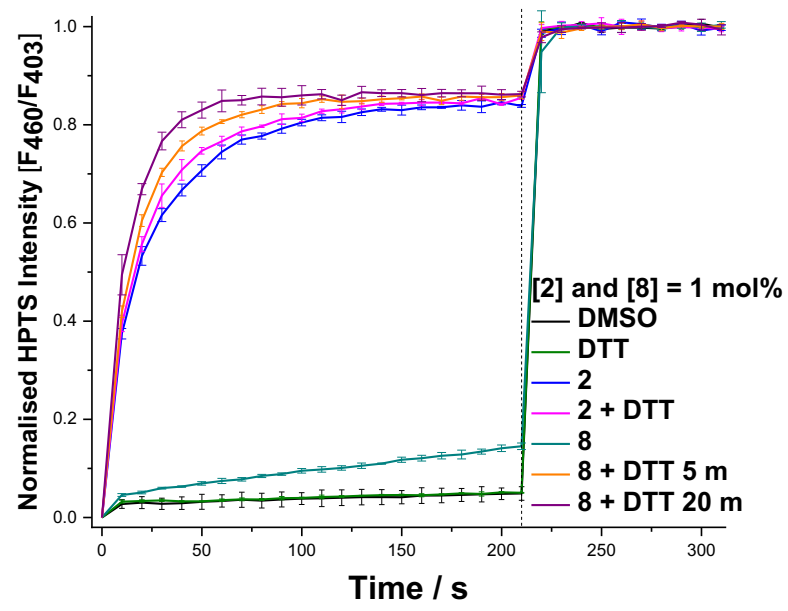

Figure S123: Observed fluorescence ratio response due to $\mathrm{H}^{+} / \mathrm{Cl}^{-}$symport or $\mathrm{Cl}^{-} / \mathrm{OH}^{-}$antiport upon reduction of complex $8(1 \mu \mathrm{M})$ by dithiothreitol DTT $(3 \mathrm{Mm})$ using $\mathrm{KCl}-\mathrm{KOH}$ assay from POPC vesicles loaded with $\mathrm{KCl}(100 \mathrm{mM})$, buffered to $\mathrm{pH} 7.0$ with HEPES $(10 \mathrm{mM})$ after 5 minutes and 20 minutes. The test complex $8(1 \mathrm{~mol} \%)$ and $\mathrm{KOH}$ were added firstly, then DTT was added at $0 \mathrm{~s}$. DMSO, DTT ( $3 \mathrm{~mol} \%$ ), parent anion transporter 2 and complex 8 (without addition of DTT) were used as controls. Detergent was added at $200 \mathrm{~s}$. Ionophore concentrations are shown as ionophore to lipid molar ratios. Error bars represent SD from at least three repeats.

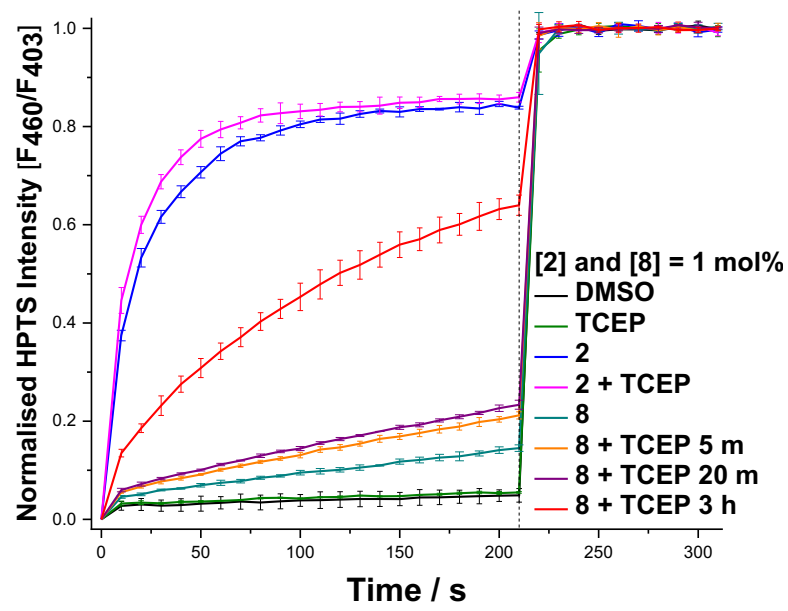

Figure S124: Observed fluorescence ratio response due to $\mathrm{H}^{+} / \mathrm{Cl}^{-}$symport or $\mathrm{Cl}^{-} / \mathrm{OH}^{-}$antiport upon reduction of complex $8(1 \mu \mathrm{M})$ by TCEP $(3 \mathrm{Mm})$ using $\mathrm{KCl}-\mathrm{KOH}$ assay from POPC vesicles loaded with $\mathrm{KCl}(100 \mathrm{mM})$, buffered to $\mathrm{pH} 7.0$ with HEPES $(10 \mathrm{mM})$ after 5 minutes, 20 minutes and 3 hours. The test complex $8(1 \mathrm{~mol} \%)$ and $\mathrm{KOH}$ were added firstly, then TCEP was added at 0 s. DMSO, TCEP ( 3 mol\%), parent anion transporter 2 and complex 8 (without addition of TCEP) were used as controls. Detergent was added at $200 \mathrm{~s}$. Ionophore concentrations are shown as ionophore to lipid molar ratios. Error bars represent SD from at least three repeats. 


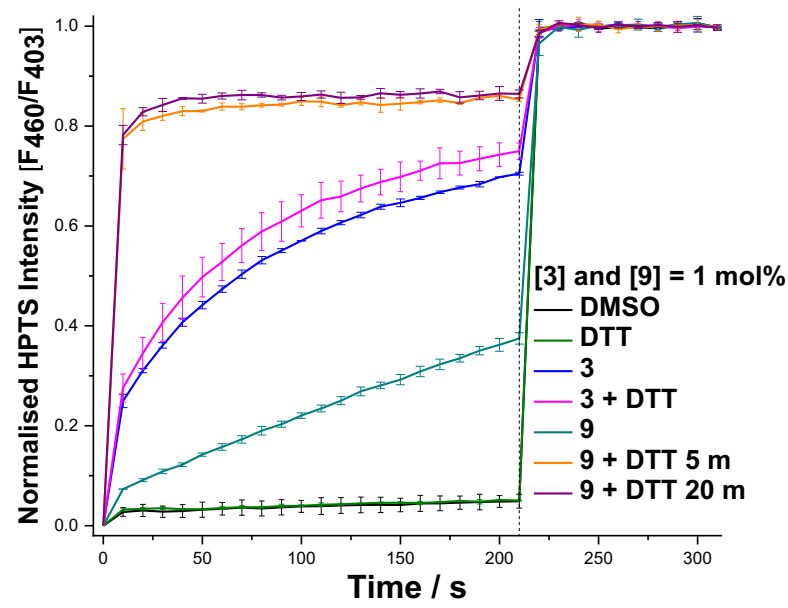

Figure S125: Observed fluorescence ratio response due to $\mathrm{H}^{+} / \mathrm{Cl}^{-}$symport or $\mathrm{Cl}^{-} / \mathrm{OH}^{-}$antiport upon reduction of complex $9(1 \mu \mathrm{M})$ by dithiothreitol DTT $(3 \mathrm{Mm})$ using $\mathrm{KCl}-\mathrm{KOH}$ assay from POPC vesicles loaded with $\mathrm{KCl}(100 \mathrm{mM})$, buffered to $\mathrm{pH} 7.0$ with HEPES $(10 \mathrm{mM})$ after 5 minutes and 20 minutes. The test complex $9(1 \mathrm{~mol} \%)$ and $\mathrm{KOH}$ were added firstly, then DTT was added at $0 \mathrm{~s}$. DMSO, DTT (3 mol\%), parent anion transporter 3 and complex 9 (without addition of DTT) were used as controls. Detergent was added at $200 \mathrm{~s}$. Ionophore concentrations are shown as ionophore to lipid molar ratios. Error bars represent SD from at least three repeats.

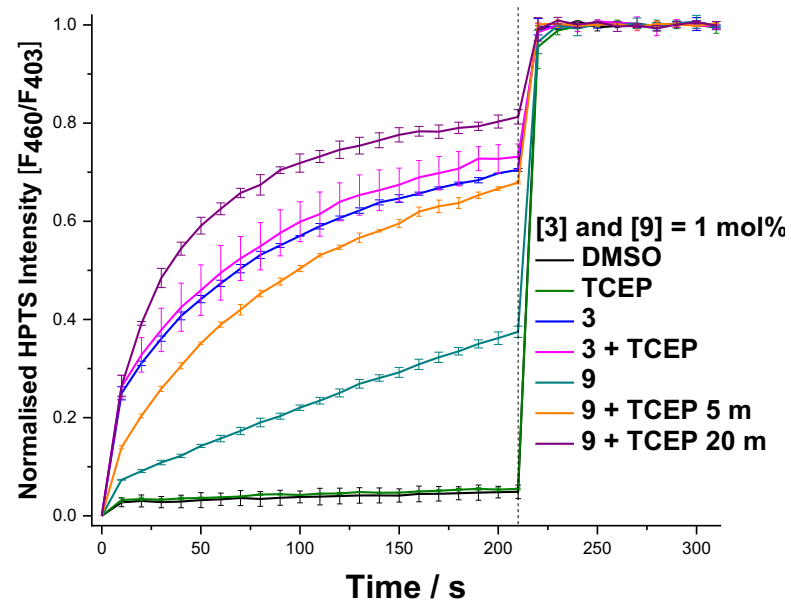

Figure S126: Observed fluorescence ratio response due to $\mathrm{H}^{+} / \mathrm{Cl}^{-}$symport or $\mathrm{Cl}^{-} / \mathrm{OH}^{-}$antiport upon reduction of complex $9(1 \mu \mathrm{M})$ by TCEP $(3 \mathrm{Mm})$ using $\mathrm{KCl}-\mathrm{KOH}$ assay from POPC vesicles loaded with $\mathrm{KCl}(100 \mathrm{mM})$, buffered to $\mathrm{pH} 7.0$ with HEPES $(10 \mathrm{mM})$ after 5 minutes and 20 minutes. The test complex $9(1 \mathrm{~mol} \%)$ and $\mathrm{KOH}$ were added firstly, then TCEP was added at $0 \mathrm{~s}$. DMSO, TCEP ( 3 mol\%), parent anion transporter 3 and complex 9 (without addition of TCEP) were used as controls. Detergent was added at $200 \mathrm{~s}$. Ionophore concentrations are shown as ionophore to lipid molar ratios. Error bars represent SD from at least three repeats. 


\section{S10: Cell viability studies:}

\section{Cell culture:}

Human colon adenocarcinoma (SW620), non-cancerous human embryonic kidney (HEK293) and non-cancerous human mammary epithelial (MCF10A) cell lines were obtained from the American Type Culture Collection (ATCC, Manassas, VA, USA). SW620 and HEK 293 cells were cultured in Dubelcco Modified Eagle Medium (DMEM) medium with $10 \%$ heat-inactivated fetal bovine serum (FBS; Life Technologies, Carlsbad, CA, USA), $100 \mathrm{U} / \mathrm{mL}$ penicillin, $100 \mu \mathrm{g} / \mathrm{mL}$ streptomycin and $2.0 \mathrm{mM} \mathrm{L}$-glutamine. The MCF-10A cell line was cultured in DMEM and Nutrient Mixture Ham's F12 (HAM) media (1:1), $5 \%$ horse serum, $10 \mu \mathrm{g} / \mathrm{mL}$ insulin (SigmaAldrich Chemical Co., St. Louis, MO, USA), $100 \mathrm{U} / \mathrm{mL}$ penicillin, $100 \mu \mathrm{g} / \mathrm{mL}$ streptomycin and $2 \mathrm{mM}$ L-glutamine, $0.5 \mu \mathrm{g} / \mathrm{mL}$ hydrocortisone (Sigma-Aldrich), $20 \mathrm{ng} / \mathrm{mL}$ epidermal growth factor (EGF, Sigma-Aldrich) and $100 \mathrm{ng} / \mathrm{mL}$ choleric toxin (Calbiochem, San Diego, CA, USA). All reagents not specified were obtained from Biological Industries, Beit Haemek, Israel. Cells were grown in a humidified atmosphere of air containing a $5 \% \mathrm{CO} 2$, at $37^{\circ} \mathrm{C}$. Cells were cultured in $10 \mathrm{~cm}$ plates and passaged 2-3 times per week using $0.05 \%$ trypsin EDTA-solution. All cells were screened for Mycoplasma sp.

\section{Cell viability assays:}

Cell viability assays were conducted using the MTT (3-(4,5-dimethylthiazol-2-yl) -2,5diphenyl-tetrazolium bromide) assay. For that purpose, $10^{4}$ cells $/ \mathrm{mL}$ were seeded in 96 well microtiter plates in $90 \mu \mathrm{L}$ per well of the respective culture medium and incubated for $24 \mathrm{~h}$ to allow cells to attach. Afterwards, they were treated with $5 \mu \mathrm{M}$ of the compounds or diluent (control cells, $1 \%$ DMSO) for $48 \mathrm{~h}$. After $48 \mathrm{~h}$ of treatment, $10 \mu \mathrm{M}$ of MTT (SigmaAldrich) diluted in $1 \times$ PBS was added to each well and assay plates were incubated for $2 \mathrm{~h}$ at $37^{\circ} \mathrm{C}$. The medium was removed and MTT formazan precipitate was dissolved in $100 \mu \mathrm{L}$ of DMSO.

The absorbance was measured at $570 \mathrm{~nm}$ using a Multiskan plate reader (Multiskan FC, Thermo Scientific). The cell viability was calculated according to the formula: viability (\%) 
$=[($ absorbance of treated wells $) /$ (absorbance of control wells $)] \times 100$. The single point experiment viabilities values were obtained using GraphPad Prism V5.0 for Windows ${ }^{\mathrm{TM}}$ (GraphPad Software, San Diego, CA, USA). All data are shown as the mean value \pm S.D. of at least three independent experiments performed in duplicate for each compound.

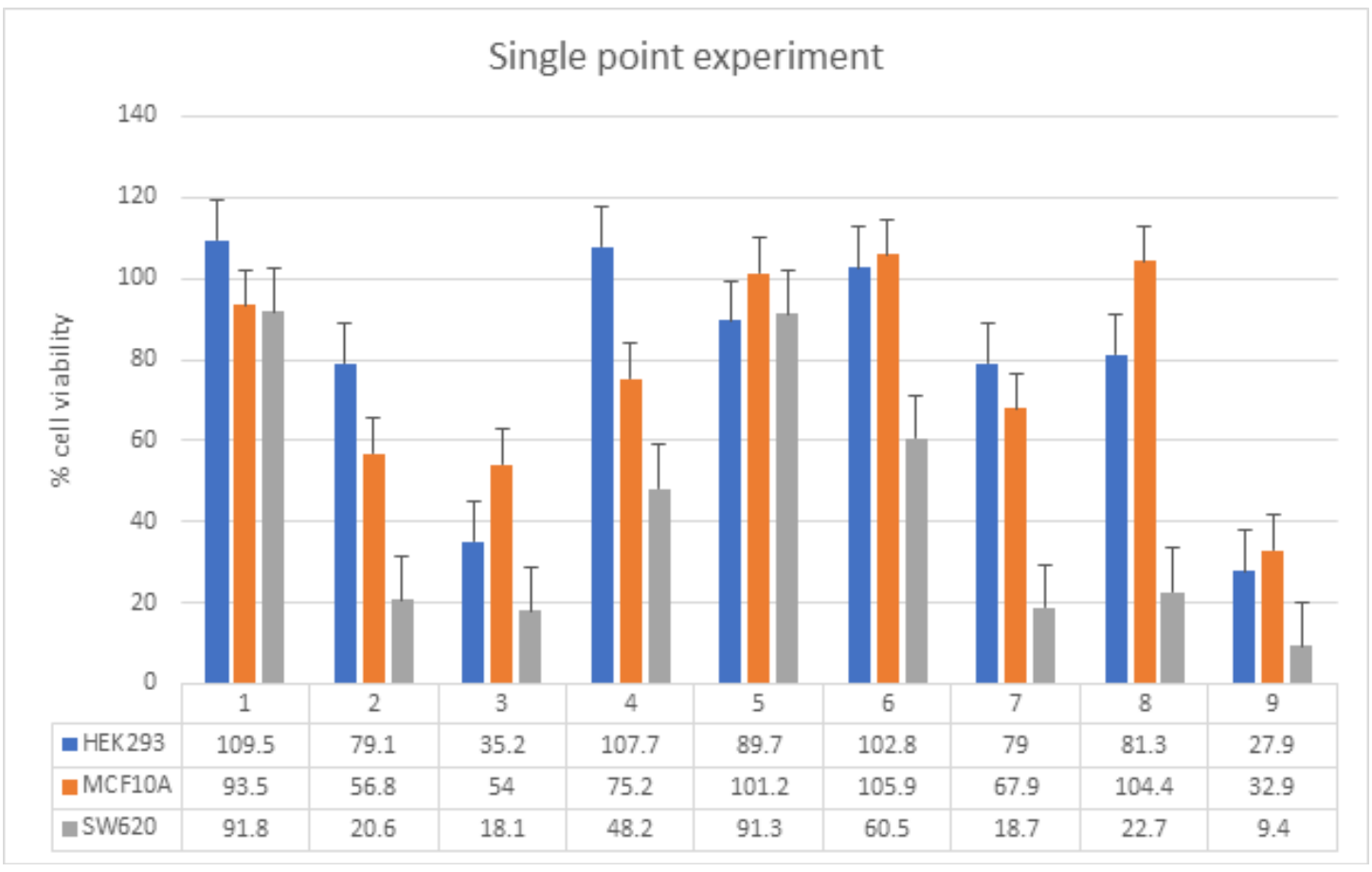

Figure S127: Cell viability in human embryonic kidney HEK293, human mammary epithelial MCF10A, and human colon adenocarcinoma SW620 cell lines in the presence of $5 \mu \mathrm{M}$ of compounds 1-9 during $48 \mathrm{~h}$. 


\section{S11: References:}

[1] a) G. Li, J. Huang, M. Zhang, Y. Zhou, D. Zhang, Z. Wu, S. Wang, X. Weng, X. Zhou, G. Yang, Chem. Commun. 2008, 38, 4564-4566; b) E. R. Parnham, R. E. Morris, Chem. Mater. 2006, 18, 4882-4887; c) H. M. Wang, C. Y. Chen, I. J. Lin, Organometallics 1999, 18, 1216-1223.

[2] A. W. Addison, P. J. Burke, J. Heterocycl. Chem. 1981, 18, 803-805.

[3] H. Goeker, M. Alp, Z. Ateş - Alagöz, S. Yıldız, J. Heterocycl. Chem. 2009, 46, 936-948.

[4] T. Zou, C. T. Lum, S. S. Chui, C. M. Che, Angew. Chem. Int. Ed. Engl. 2013, 52, 29302933.

[5] L. J. Bourhis, O. V. Dolomanov, R. J. Gildea, J. A. Howard, H. Puschmann, Acta Crystallogr. Sect. A: Found. Adv. 2015, 71, 59-75.

[6] O. V. Dolomanov, L. J. Bourhis, R. J. Gildea, J. A. Howard, H. Puschmann, J. Appl. Crystallogr. 2009, 42, 339-341.

[7] G. M. Sheldrick, Acta Crystallogr. Sect. A: Found. Crystallogr. 2008, 64, 112-122.

[8] a) S. N. Berry, V. Soto-Cerrato, E. N. Howe, H. J. Clarke, I. Mistry, A. Tavassoli, Y.-T. Chang, R. Pérez-Tomás, P. A. Gale, Chem. Sci. 2016, 7, 5069-5077; b) L. A. Jowett, E. N. Howe, X. Wu, N. Busschaert, P. A. Gale, Chem. Eur. J. 2018, 24, 10475-10487; c) X. Wu, L. W. Judd, E. N. Howe, A. M. Withecombe, V. Soto-Cerrato, H. Li, N. Busschaert, H. Valkenier, R. Pérez-Tomás, D. N. Sheppard, Chem 2016, 1, 127-146.

[9] E. N. W. Howe, P. A. Gale, J. Am. Chem. Soc. 2019, 141, 10654-10660.

[10] X. Wu, P. Wang, P. Turner, W. Lewis, O. Catal, D. S. Thomas, P. A. Gale, Chem 2019, $5,1210-1222$. 
